\title{
EFFECT OF HYOROXYPROPYLATION ON THE STRUCTURE AND PHYSICOCHEMICAL PROPERTIES OF NATIVE, DEFATTED AND HEAT-MOISTURE TREATED POTATO STARCHES
}

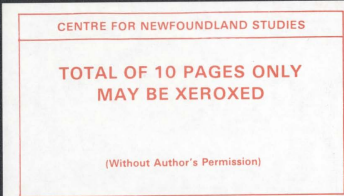

CHANDANI PERERA 


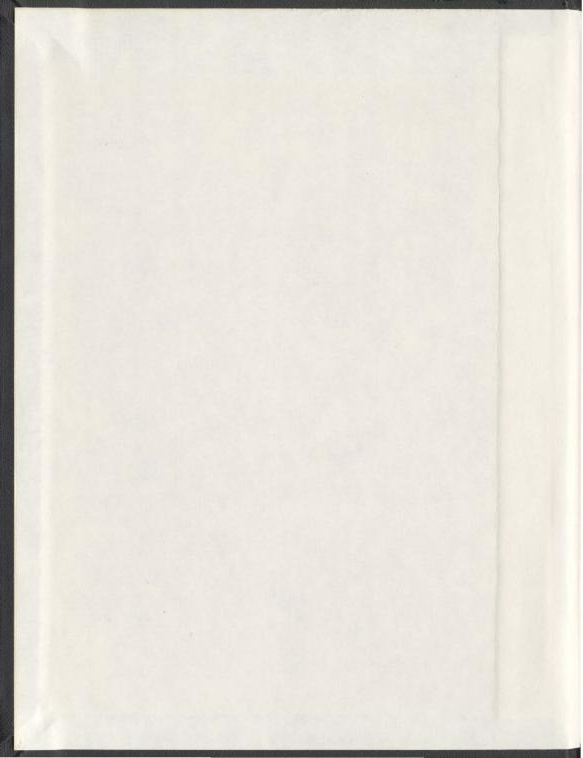




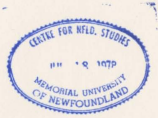





\section{EFFECT OF HYDROXYPROPYLATION ON THE STRUCTURE AND PHYSICOCHEMICAL PROPERTIES OF NATIVE, DEFATTED AND HEAT-MOISTURE TREATED POTATO STARCHES}

BY

\section{CHANDANI PERERA}

A thesis submitted to the School of Graduate Studies in partial fulfillment of the requirements for the degree of

Doctor of Philosophy

Department of Biochemistry

Memorial University of Newfoundland

June 1998

St. John's

Newfoundland

Canada 


\begin{abstract}
Native potato starch was physically modified by heat-moisture treatment $\left(100^{\circ} \mathrm{C}, 16 \mathrm{~h}, 30 \%\right.$ moisture) and defatting $(75 \%, n$-propanol water, $7 \mathrm{~h})$. The changes in structure and physicochemical properties on heat-moisture treatment and defatting were monitored by scanning electron microscopy (SEM), X-ray diffraction, differential scanning calorimetry (DSC). Brabender viscosities, swelling factor (SF) and amylose leaching (AML). SEM showed that neither defatting nor heat-moisture treatment altered the size, shape or the surface appearance of the native starch granule. Heat-moisture treatment decreased $\mathrm{X}$ ray diffraction intensities and altered the 'B' type $X$-ray diffraction pattern to 'A+B'. The decrease in $\mathrm{X}$-ray intensities on heat-moisture treatment is indicative of crystallite disruption and/or rearrangement of double helices. The gelatinization enthalpy $(\mathrm{AH})$, Brabender viscosity (at $95^{\circ} \mathrm{C}$ ), SF and $\mathrm{AML}$ decreased on heat-moisture treatment, whereas gelatinization transition temperatures (GTT), and thermal stability increased. Defatting increased the $X$ ray diffraction intensities and altered the X-ray pattem from 'B' to 'A+B'. The increased $\mathrm{X}$-ray intensities on defatting is indicative of interactions between amylose - amylose (AM-AM), amylopectin - amylopectin (AMP-AMP) and amylose - amylopectin (AM-AMP) chains. These interactions in tum, increased GTT, $\Delta \mathrm{H}$ and thermal stability. However, SF, AML and Brabender viscosity (at $\left.95^{\circ} \mathrm{C}\right)$ decreased on defatting.
\end{abstract}


The reagents $\left(\mathrm{NaOH}\right.$ and $\mathrm{Na}_{2} \mathrm{SO}_{4}$ ) used during hydroxypropylation did not alter granule morphology and AML in native, defatted and heat-moisture treated starches. X- ray diffraction pattems of native and defatted starches changed on alkaline treatment, whereas that of heat-moisture treated starch remained unaltered. These changes reflected double helical disruption (within the amorphous regions), and altered crystallite orientation. In all three starches, alkaline conditions decreased $\Delta \mathrm{H}$ and Brabender viscosity (at $95^{\circ} \mathrm{C}$ ), and increased SF. The extent of the above changes followed the order : native > defatted > heat-moisture treated. Gelatinization transition temperatures remained unchanged on alkaline treatment.

Native, defatted and heat-moisture treated starches were converted to a range of hydroxypropyl derivatives using propylene oxide (at concentrations ranging from $2-25 \% \mathrm{v} / \mathrm{w})$. At $2 \%(\mathrm{v} / \mathrm{w})$ propylene oxide, all three starches showed similar molar substitution (MS 0.05). However, at 5-25\%(v/w) propylene oxide, the accessibility of hydroxypropyl groups into the starch granule followed the order : heat-moisture treated > native > defatted. This showed that the degree of accessibility of hydroxypropyl groups into the granule interior is dependent upon granule crystallinity. In all three starches, an increase in MS progressively decreased GTT, $\triangle H$ and AML. The influence of MS on SF of hydroxypropylated native, defatted and heat-moisture treated starches was due to the interplay that occurs between hydrogen bond disruption (due to hydroxypropyl groups) within the amorphous regions, and the increased interactions that occur between starch 
chains during defatting and heat-moisture treatment. Pasting temperatures of all starches decreased with increased MS. In defatted starch, Brabender viscosity (at $95^{\circ} \mathrm{C}$ ) progressively increased with increase in MS. However, in native and heat-moisture treated starches, Brabender viscosity (at $95^{\circ} \mathrm{C}$ ) began to decrease (due to granule disruption) at MS levels beyond 0.18 and 0.20 , respectively.

Enzyme digestibility studies showed that both defatting (hot $75 \% \mathrm{n}$ propanol, 0-7 h) and heat-moisture treatment $\left(100^{\circ} \mathrm{C}, 30 \%\right.$ moisture, 0-16 h) increased the susceptibility of potato starch granules towards hydrolysis by porcine pancreatic $\alpha$ - amylase. These differences were attributed to structural changes that occurred within the amorphous and crystalline regions of the starch granule during defatting and heat-moisture treatment. However, hydrolysis decreased (due to formation of new crystallites) when heat-moisture treatment and defatting were continued beyond $8 \mathrm{~h}$ and $9 \mathrm{~h}$, respectively.

Native, defatted $(7 \mathrm{~h})$ and heat-moisture treated $(16 \mathrm{~h})$ potato starches were hydroxypropylated (to different levels of MS) with propylene oxide (2 $20 \%$ ). The results showed that the reagents $\left(\mathrm{NaOH}\right.$ and $\left.\mathrm{Na}_{2} \mathrm{SO}_{4}\right)$ used during hydroxypropylation increased the susceptibility of the above starches (native > defatted $>$ heat-moisture treated) towards hydrolysis by $\alpha$-amylase. Addition of propylene oxide (hydroxypropylation) to alkali treated starches, further enhanced their susceptibility towards $\alpha$-amylase. However, granule susceptibility towards 
$\alpha$-amylase did not increase exponentially with increase in MS. The extent of hydrolysis began to decrease at MS levels of 0.29 (native), 0.28 (heat-moisture treated) and 0.26 (defatted). The decruase in hydrolysis at higher MS levels is indicative of the steric effect imposed by bulky hydroxypropyl groups on the accessibility of $\alpha$-amylase towards the glycosidic bonds of amylose and amylopectin.

The retrogradation properties of potato starch gels (stored for $24 \mathrm{~h}$ at $4^{\circ} \mathrm{C}$ and then for 34 days at $40^{\circ} \mathrm{C}$ ) before and after physical (defatting and heatmoisture treatment), and chemical (hydroxypropylation) modification were monitored using turbidity measurements, SEM, DSC, $\mathrm{X}$-ray diffraction and enzyme susceptibility. Turbidity development in native, defatted and heatmoisture treated starch pastes during storage $\left(4^{\circ} \mathrm{C}\right.$ for $24 \mathrm{~h}$ and then at $40^{\circ} \mathrm{C}$ for 34 days) followed the order : native > defatted > heat-moisture treated. In all three starches, the highest rate of turbidity development was observed during the first $24 \mathrm{~h}$ of storage (at $4^{\circ} \mathrm{C}$ ). The above results in conjunction with SEM observations showed that turbidity development is influenced by the interaction between leached starch components (AM-AM, AM-AMP, AMP-AMP), and interaction between granule remnants and leached starch components (amylose and amylopectin). In alkali treated gelatinized native, defatted and heat-moisture treated starch pastes, turbidity development (native $>$ defatted $>$ heat-moisture treated) was influenced by aggregation of granule remnants. In native, defatted and heat-moisture treated starch pastes, both the rate and extent of turbidity 
development (on storage) decreased after hydroxypropylation. This decrease was due to the interplay of : 1) steric effects imposed by hydroxypropyl groups on chain aggregation, 2) aggregation between small granule remnants, and 3) settling of large granule remnants beneath the path of the spectrophotometer beam.

The intensity (at 5.2A ) of the 'B' type $\mathrm{X}$-ray pattern of gelatinized pastes of native, defatted and heat-moisture treated starches increased during storage (native > defatted > heat-moisture treated). The same ' $\mathrm{B}$ ' pattern was also evident (at the end of the storage period) after alkali treatment and hydroxypropylation of the above starches. However, the peak at 5.2A was reduced in intensity after alkaline treatment and hydroxypropylation. The extent of the decrease being greater in the latter.

Fresh pastes of gelatinized native, defatted and heat-moisture treated potato starches were hydrolyzed by porcine pancreatic $\alpha$-amylase nearly to the same extent $\left(75.3-77.2 \%\right.$ ). Storage (at $4^{\circ} \mathrm{C}$ for $24 \mathrm{~h}$ ) of the above gelatinized pastes decreased (native > defatted > heat-moisture treated) their susceptibility towards $\alpha$-amylase. However, storage times longer than $24 \mathrm{~h}$ did not seem to have any further influence on the enzyme susceptibility of the starch gels. Alkaline treatment increased the susceptibility of freshly gelatinized starch pastes (native > defatted > heat-moisture treated) towards hydrolysis by $\alpha$-amylase. However, storage $\left(24 \mathrm{~h}\right.$ at $\left.4^{\circ} \mathrm{C}\right)$ of alkali treated starch gels, decreased their susceptibility towards hydrolysis by a-amylase. Hydroxypropylation decreased 
the accessibility of $\alpha$-amylase towards the glycosidic linkages of freshly gelatinized pastes of native, defatted and heat-moisture treated starches (defatted > native > heat-moisture treated). However, the extent of this decrease was not altered during storage (at $4^{\circ} \mathrm{C}$ for $24 \mathrm{~h}$ ).

The retrogradation endotherm (monitored by DSC) of starch gels (native, defatted and heat-moisture treated) occurred after 2 days of storage $\left(4^{\circ} \mathrm{C}\right.$ for 1 day and then at $40^{\circ} \mathrm{C}$ for 1 day). A similar trend was also observed after alkaline treatment. However, hydroxypropylated native, defatted and heat-moisture treated starch geis [at nearty the same MS level $(0.10-0.11)$ ], showed a measurable retrogradation endotherm only after 7 days $\left(4^{\circ} \mathrm{C}\right.$ for 1 day and then at $40^{\circ} \mathrm{C}$ for 6 days). This showed that hydroxypropyl groups are effective in hindering starch chain realignment during gel storage. Both alkaline treatment and hydroxypropylation decreased the retrogradation enthalpies of native. defatted and heat-moisture treated starch gels. 


\section{ACKNOWLEDGMENTS}

I have greatly appreciated the opportunity to work with Dr. R. Hoover (supervisor) and Dr. A.M. Martin (co-supervisor). They have been patient and supplied me with encouragement and motivation to persevere.

I also appreciate Dr. J. Banoub, Dr. A. D. Rahimtula and Dr. S.S Mookerjea for serving on my supervisory committee and for the time and effort that they expended on my behalf.

I would like to acknowledge the Department of Biochemistry and the school of Graduate studies for providing financial assistance. Financial support from Dr. R. Hoover and Dr. A.M. Martin is also acknowledged.

Thanks are also extended to Dr. K. Keough and Dr. T.R. Patel for providing me access to their laboratory facilities. I also thank my fellow graduate students (Mr. E. Acheampong and Mr. N. Senanayake), with whom I have been privileged to associate these past years. As we learned together, their camaraderie has made life here interesting and most enjoyable. 


\section{TABLE OF CONTENTS}

Abstract

Acknowledgments

Table of contents

List of Figures

List of Tables

xiv

List of Abbreviations

xvii

Chapter 1. Introduction

$x \times$

Chapter 2. Literature Review

2.1 Starch-Introduction

2.2 Starch Biosynthesis

2.3 Starch Production and uses 13

2.4 Structure of starch

2.4.1 Major components $\quad 15$

2.4.1.1 Amylose $\quad 15$

2.4.1.1.1 Structure $\quad 15$

2.4.1.1.2 Conformation $\quad 17$

2.4.1.2 Amylopectin $\quad 23$

2.4.2 Minor components of starch 32

2.4.2.1 Proteins $\quad 32$

2.4.2.2 Lipids $\quad 33$

2.4.2.2.1 Amylose - lipid inclusion complex $\quad 35$

2.4.2.2.2 Occurrence of amylose -lipid complex $\quad 35$

2.4.2.2.3 Stability of amylose -lipid complex $\quad 42$

2.4.2.2.4 Thermal and rheological properties of amylose - lipid complex $\quad 42$

2.4.3 Super molecular order of starch granule 45

2.4.4 Molecular organization of crystalline regions $\quad 51$

2.4.5 Crystal structure of ' $A$ ' and ' $B$ ' starches 55

2.4.6 Amorphous region of the granule 63

2.5 Starch properties 66

2.5.1 Gelatinization $\quad 66$

2.5.1.1 Factors influencing gelatinization $\quad 77$

2.5.1.1.1 Botanical source 77

2.5.1.1.2 Heating rate $\quad 79$

2.5.1.1.3 Lipids $\quad 79$

2.5.1.1.4 Sugars $\quad 80$ 
2.5.1.1.5 Starch modification

2.5.2.1 Amylose gelation 84

2.5.2.2 Amylopectin gelation 87

2.5.2.3 Retrogradation of starch 92

2.5.2.4 Factors influencing retrogradation 95

2.5.2.4.1 Botanical source 95

2.5.2.4.2 Storage temperature 96

2.5.2.4.3 Moisture content of gels 98

2.5.2.4.4 Lipids 99

2.5.2.4.5 Sugars 100

2.5.2.4.6 Salts $\quad 102$

2.5.2.4.7 Hydrocolloids $\quad 103$

2.5.2.4.8 Physical modification $\quad 103$

2.5.2.4.9 Chemical modification $\quad 105$

2.5.3 Starch digestibility 106

2.6 Starch modification $\quad 109$

2.6.1 Physical modification 109

2.6.1.1 Heat-moisture treatment $\quad 110$

2.6.1.2 Defatting 114

2.6.2 Chemical modification $\quad 117$

2.6.2.1 Hydroxypropylation $\quad 121$

Chapter 3. Materials and Methods $\quad 128$

$\begin{array}{ll}3.1 \text { Materials } & 128\end{array}$

3.2 Methods 128

3.2.1 Starch isolation and purification 128

3.2.2 Chemical composition of starch 129

3.2.2.1 Moisture content 129

3.2.2.2 Nitrogen content 129

3.2.2.3 Ash content 130

3.2.2.4 Lipid content 130

3.2.2.5 Amylose content 131

3.2.2.5.1 Chrastil's method 131

3.2.2.6 Estimation of starch damage $\quad 132$

3.2.3 Starch modification 133

3.2.3.1 Preparation of defatted potato starch 133

3.2.3.2 Preparation of heat-moisture treated potato starch 
3.2.3.3 Preparation of hydroxypropyl potato starch 134

3.2.3.3.1 Determination of molar substitution 134

3.2.4 Determination of physicochemical properties 136

3.2.4.1 Scanning electron microscopy 136

3.2.4.2X-ray diffraction $\quad 136$

3.2.4.3 Differential scanning calorimetry $\quad 137$

3.2.4.4 Swelling factor $\quad 137$

3.2.4.5 Extent of amylose leaching $\quad 139$

3.2.4.6 Brabender viscosity measurements $\quad 139$

3.2.5 Starch digestibility 140

3.2.5.1 Preparation of defatted starch $\quad 140$

3.2.5.2 Preparation of heat-moisture treated starch $\quad 140$

3.2.5.3 Scanning electron microscopy $\quad 140$

3.2.5.4 Enzyme hydrolysis $\quad 141$

3.2.6 Starch retrogradation 142

3.2.6.1 Turbidity measurements $\quad 142$

3.2.6.2 Gel preparation $\quad 142$

3.2.6.3 Gel powder preparation $\quad 143$

3.2.6.4 Scanning electron microscopy of gels 143

3.2.6.5 Differential scanning calorimetry 144

3.2.6.6 Enzyme hydrolysis of gels $\quad 145$

3.2.7 Statistical analysis $\quad 145$

Chapter 4. Results and Discussion 146

4.1 Chemical composition of native potato starch 146

4.2 Effect of defatting and heat-moisture treatment on the structure and physicochemical properties of native potato starch 148

4.2.1 Morphological granular characteristics 148

4.2.2X-ray diffraction 148

4.2.3 Swelling factor and amylose leaching $\quad 153$

4.2.4 Differential scanning calorimetry 156

4.2.5 Brabender viscosity measurements $\quad 159$

4.3 Effect of alkali treatment on the structure and properties of native, defatted and heat-moisture treated starches 162

4.3.1 Morphological granular characteristics 162

4.3.2 X-ray diffraction 163

4.3.3 Swelling factor and amylose leaching $\quad 163$

4.3.4 Differential scanning calorimetry 163

4.3.5 Brabender viscosity measurements 168

4.4 Effect of defatting and heat-moisture treatment on the hydroxypropylation of native potato starch

4.5 The effect of hydroxypropylation on the structure, thermal and pasting properties of native, defatted and heat-moisture treated potato starches 
4.5.1 Morphological granular characteristics $\quad 176$

4.5.2 X-ray diffraction 176

4.5.3 Swelling factor $\quad 177$

4.5.4 Amylose leaching 180

4.5.5 Differential scanning calorimetry $\quad 180$

4.5.6 Brabender viscosity measurements $\quad 184$

4.6 Starch digestibility by porcine pancreatic $\alpha$-amylase $\quad 186$

4.6.1 Enzyme hydrolysis of native, defatted and heatmoisture treated potato starches 186

4.6.2 Influence of alkali on enzyme hydrolysis of native, defatted and heat-moisture treated potato starches 198

4.6.3 The influence of level of hydroxypropylation on enzyme hydrolysis of native, defatted and heat-moisture treated potato starches

4.7 Retrogradation studies on potato starches

4.7.1 Turbidimetric and structural analysis

4.7.1.1 Initial turbidity and SEM of fresh pastes of native, defatted and heat-moisture treated starches

4,7.1.2 Initial turbidity and SEM of alkali treated native, defatted and heat-moisture treated starches

4.7.1.3 Initial turbidity and SEM of hydroxypropylated native, defatted and heat-moisture treated starches 213

4.7.1.4 Turbidity and SEM of fresh pastes of native, defatted and heat-moisture treated starch pastes during storage

4.7.1.5 Turbidity and SEM of alkali treated native, defatted

and heat-moisture treated starch pastes during storage

4.7.1.6 Turbidity and SEM of hydroxypropylated native, defatted and heat-moisture treated starch pastes during storage

4.7.2 Enzyme digestibility

4.7.2.1 Enzyme digestibility of fresh and stored pastes of native, defatted and heat-moisture treated starches

4.7.2.2 Enzyme digestibility of fresh and stored pastes of alkali treated native, defatted and heat-moisture treated starches

4.7.2.3 Enzyme digestibility of fresh and stored pastes of hydroxypropyl native, defatted and heat-moisture treated starches

4.7.3 X-ray diffraction

4.7.3.1 X-ray diffraction of fresh and stored gels of native, defatted and heat-moisture treated starches 
4.7.3.2 X-ray diffraction of fresh and stored gels of alkali treated native, defatted and heat-moisture treated starches

4.7.3.3 X-ray diffraction of fresh and stored gels of hydroxypropyl native, defatted and heat-moisture treated starches

4.7.4 Differential scanning calorimetry

4.7.4.1 DSC parameters of native, defatted and heat-moisture treated gels after storage

4.7.4.2 DSC parameters of alkali treated native, defatted and heat-moisture treated gels after storage

4.7.4.3 DSC parameters of hydroxypropyl native, defatted and heat-moisture treated gels after storage

Chapter 5. Summary and Conclusion

5.1 Directions for future research 


\section{LIST OF FIGURES}

Figure

2.1 Metabolic pathway for starch synthesis

$2.2{ }^{13} \mathrm{C}$ CP/MAS NMR spectra of 'A' and 'B' type amylose

2.3 Models proposed for the amylose in aqueous solution 19

2.4 Schematic illustration of amylose-lipid complex 22

$2.5 \mathrm{X}$ - ray diffraction pattern of ' $\mathrm{V}$ amylose 24

$\begin{array}{lll}2.6 & \text { Models proposed for amylopectin } & 27\end{array}$

2.7 Chain distribution of potato, waxy rice starches and the proposed model for amylopectin

2.8 Proposed models for 'A' and ' $B$ ' type amylopectin

$2.9{ }^{13} \mathrm{C}$ CPMMAS NMR spectra of ' $\mathrm{V}$ amylose

2.10 DSC thermal curve of a cereal starch showing different melting transitions and the corresponding structural domains

2.11 Structures in binary lipid-water systems

2.12 Schematic modeis of starch granule

2.13 Inner structure of starch granule showing crystalline and amorphous regions

2.14 Schematic model for the arrangement of amylopectin in potato starch

2.15 X-ray diffraction patterns of 'A', 'B' and ' $C$ ' starches

2.16 Double helix packing arrangement in 'A' and ' $B$ ' type unit cells

2.17 Structure of 'A' and ' $B$ ' unit cells

$2.18{ }^{13} \mathrm{C}$ CP/MAS NMR spectra of amorphous and crystalline 'A' type and ' $B$ ' type starches 
2.19 The transition from ' $B$ ' starch to 'A' starch

2.20 Schematic presentation of the changes which occur in the starch granule during gelatinization

2.21 DSC thermograms of starches at various volume fractions of water $\mathbf{7 1}$

2.22 Thermal profiles of rice starch showing both glass transition and melting transition

2.23 DSC thermograms of starches at various water contents

2.24 Mechanisms of starch retrogradation

82

2.25 Continuous model for amylose gel

88

2.26 DSC thermograms of waxy starch and waxy amylopectin gels

90

2.27 Effect of storage temperature on crystallization of partially crystalline polymers

2.28 Starch hydrolysis by $\alpha$-amylase : mechanism

2.29 Glucose units substituted with hydroxypropyl groups showing molar substitution

4.1 Scanning electron micrographs of native, defatted and heatmoisture treated potato starch granules

4.2 X-ray diffraction pattems of native, defatted and heat-moisture treated potato starches

4.3 X-ray diffraction patterns of native, defatted and heat-moisture treated potato starches after alkaline treatment

4.4 The level of molar substitution of native, heat-moisture treated and defatted potato starch by hydroxypropyl groups

4.5 Influence of time of defatting and heat-moisture treatment on MS level

4.6 Scanning electron micrographs of native defatted, and heatmoisture treated potato starch granules hydrolyzed by porcine pancreatic $\alpha$-amylase for $72 \mathrm{~h}$. 
4.7 X-ray diffraction patterns of potato starches heat-moisture treated for different time periods

4.8 The influence of defatting time on the extent of lipid removal from native potato starch

4.9 X-ray diffraction patterns of potato starches defatted for different time periods

4.10 Time course of turbidity development in native potato starch stored at $4^{\circ} \mathrm{C}$ and $40^{\circ} \mathrm{C}$

4.11 Time course of turbidity development in defatted potato starch stored at $4^{\circ} \mathrm{C}$ and $40^{\circ} \mathrm{C}$

4.12 Time course of turbidity development in heat-moisture treated potato starch stored at $4^{\circ} \mathrm{C}$ and $40^{\circ} \mathrm{C}$

4.13 Scanning electron micrographs of freshly gelatinized and stored native, defatted and heat-moisture treated potato starch pastes

4.14 Scanning electron micrographs of freshly gelatinized and stored alkali treated native potato starch pastes

4.15 Scanning electron micrographs of freshly gelatinized and stored alkali treated defatted potato starch pastes

4.16 Scanning electron micrographs of freshly gelatinized and stored alkali treated heat-moisture treated potato starch pastes

4.17 Scanning electron micrographs of freshly gelatinized and stored hydroxypropyl potato starch pastes

4.18 X-ray diffraction patterns of freshly gelatinized and stored pastes of untreated, alkali treated and hydroxypropylated native potato starches

4.19 X-ray diffraction patterns of freshly gelatinized and stored pastes of untreated, alkali treated and hydroxypropylated defatted potato starches

4.20 X-ray diffraction patterns of freshly gelatinized and stored pastes of untreated, alkali treated and hydroxypropylated heat-moisture treated potato starches 


\section{LIST OF TABLES}

Table

2.1 Size and shape of starch granules from different botanical sources 9

2.2 Characteristics of amylose and amylopectin 10

2.3 Properties of amylose from different botanical sources 16

2.4 Properties of amylopectin from different botanical sources 25

2.5 Lipid-complexed and lipid-free amylose in barley starches 38

2.6 Percent crystallinity of ' $A$ ', 'B' and ' $C$ ' type starches 54

2.7 Gelatinization parameters of some starches 78

2.8 Properties and applications of chemically modified starches 118

4.1 Proximate composition of native potato starch 147

4.2 X-ray diffraction intensities of the major peaks of native, defatted and heat-moisture treated potato starches

4.3 Swelling factor of native, defatted and heat-moisture treated potato starches

4.4 Amylose leaching of native, defatted and heat-moisture treated potato starches

4.5 Gelatinization parameters of native, defatted and heat-moisture treated starches

4.6 Pasting properties of native, defatted and heat-moisture treated potato starches

4.7 X-ray diffraction intensities of the major peaks of native, defatted and heat-moisture treated potato starches before and after alkali treatment

4.8 Swelling factor of native, defatted and heat-moisture treated potato starches before and after alkaline treatment 
4.9 Amylose leaching (at $90^{\circ} \mathrm{C}$ ) of native, defatted and heat-moisture treated potato starches before and after alkaline treatment

4.10 Gelatinization parameters of native, defatted and heat-moisture treated starches before and after alkaline treatment

4.11 Pasting properties of native, defatted and heat-moisture treated starches before and after alkaline treatment

4.12 Swelling factor of hydroxypropyl native, defatted and heat-moisture treated potato starches

4.13 Amylose leaching of hydroxypropyl native, defatted and heat-moisture treated potato starches

4.14 Gelatinization parameters of hydroxypropyl native, defatted and heat-moisture treated potato starches

4.15 Pasting properties of hydroxypropyl native, defatted and heat-moisture treated potato starches

4.16 Hydrolysis of native, defatted and heat-moisture treated potato starches by porcine pancreatic $\alpha$-amylase

4.17 Effect of time of heat-moisture treatment on hydrolysis of potato starch by porcine pancreatic $\alpha$-amylase

4.18 Effect of time of defatting on hydrolysis of potato starch by porcine pancreatic $\alpha$-amylase

4.19 X-ray diffraction intensities of the $5.2 \AA$ peak with time course of heat-moisture treatment

4.20 Hydrolysis of native, defatted and heat-moisture treated potato starches by porcine pancreatic $\alpha$-amylase before and after hydroxypropylation

4.21 $\alpha$-amylase hydrolysis of freshly gelatinized and stored pastes of native, defatted and heat-moisture treated starches

4.22 $\alpha$-amylase hydrolysis of freshly gelatinized and stored pastes of alkali treated native, defatted and heat-moisture treated starches 
4.23 $\alpha$-amylase hydrolysis of freshly gelatinized and stored pastes of hydroxypropyl native, defatted and heat-moisture treated starches

4.24 X-ray diffraction intensities (at 5.2A) of stored potato starch gels

4.25 DSC parameters of native, defatted and heat-moisture treated potato starches after gelatinization and storage

4.26 DSC parameters of alkali treated native, defatted and heat-moisture treated potato starches

4.27 DSC parameters of hydroxypropyl native, defatted and heat-moisture treated potato starches 


\section{LIST OF ABBREVIATIONS}

$v$
$\Delta H$
$\Delta H_{R}$
AM
AML
AMP
BU
C*
CL
CP MAS NMR
CTAB
db
DP
DPn
DPw
DSC
F-AM
FTIR
GMP
GMS
HPLC
L-AM
MG
MS
NIR
PPA
PW
SF
SP
SSL
To
Tp
TC
TC-To
Tg
w/v

- Volume fraction

- Enthalpy of gelatinization

- Enthalpy of retrogradation

- Amylose

- Amylose leaching

- Amylopectin

- Brabender units

- Entanglement concentration

- Chain length

- Cross polarization magic angle spinning nuclear magnetic resonance

- Cetyltrimethylammonium bromide

- Dry basis

- Degree of polymerization

- Degree of polymerization - number (average)

- Degree of polymerization - weight (average)

- Differential scanning calorimetry

- Lipid free amylose

- Fourier transform infrared spectroscopy

- Glyceryl monopaimitate

- Glyceryl monostearate

- High performance liquid chromatography

- Lipid amylose

- Monoacylglycerol

- Molar substitution

- Near infrared reflectance spectroscopy

- Porcine pancreatic $\alpha$-amylase

- Propanol water

- Swelling factor

- Swelling power

- Sodium stearoyl-2- lactylate

- Onset of gelatinization temperature

- Peak gelatinization temperature

- Conclusion of gelatinization temperature

- Gelatinization transition temperature range

- Glass transition temperature

-Weight / volume 


\section{DEDICATED TO MY LOVING PARENTS}




\section{CHAPTER 1}

\section{INTRODUCTION}

Modified starches are important in processed foods because of their improved functional properties over their unmodified counterparts. Reaction of starch with propylene oxide to form the hydroxypropyl starch derivatives is used primarily in the food industry. This modification process improves the freeze thaw stability, clarity and textural properties of the starch paste. Hydroxypropyl groups are hydrophilic in nature and when introduced into the starch granule, weaken or strain the internal bond structure holding the granule together. Hydroxypropylated starches have been shown to alter physicochemical properties compared to their native starches (Butler et al., 1986; Hoover et al. 1988; Kim \& Eliasson, 1993), primarily depending on the molar substitution (MS).

Hood \& Mercier (1978) have shown by enzyme hydrolysis and gel chromatography, that in manioc starch the hydroxypropyl groups in amylose were distributed at/or near the reducing end or along the entire amylose molecule. Kim et al. (1992) have shown by light microscopy studies, that in potato starch granule, hydroxypropyl groups are mainly distributed in the central region. Previous studies have shown that physical modification alters starch granule structure. This suggests that the distribution of hydroxypropyl groups within the granule interior could change on physical modification.

Presently hydroxypropyl starches for commercial purposes are produced by reaction of a highly concentrated slurry of starch granules with propylene 
oxide under alkaline conditions $\left(\mathrm{NaOH}\right.$ and $\left.\mathrm{Na}_{2} \mathrm{SO}_{4}\right)$. These alkaline conditions increase the reaction efficiency of hydroxypropylation. The changes in physicochemical properties on hydroxypropylation have been attributed solely to the level of MS. No attempt has been made to investigate the possibility that the alkaline conditions could also influence starch properties.

The reactivity and the mode of action of $\alpha$-amylase have been primarily studied on unmodified starches from various botanical sources, and the results have shown that the action of $\alpha$-amylase is influenced by granule structure. It has been postulated (Marsden \& Gray, 1986; Franco et al., 1988) that $\alpha$-amylase preferentially hydrolyzes amorphous regions of the starch granule. However, studies have not been conducted to examine how the arrangement of starch chains within the amorphous regions of the starch granule influences the degree of accessibility of $\alpha$-amylase into the granule interior. Previous studies (Hoover \& Vasanthan, 1994a,b; Hoover \& Manuel, 1996a) have shown that starch chain interactions occur within the amorphous region during thermal treatment. Furthermore, chemical modification has been shown (Mohd Azemi \& Wootton, $1985 ; 1995)$ to reduce the accessibility of $\alpha$-amylase into the amorphous regions. Thus, a comparative study of $\alpha$-amylolysis of native starches with their physically and chemically modified counterparts may provide a deeper insight into the mechanism of $\alpha$-amylolysis. 
Starch gelatinization is a process that takes place when starch granules are heated in the presence of water, resulting in the disruption of molecular order within the starch granule. The process is manifested by irreversible changes in granular swelling, crystallite melting, loss of birefringence and starch solubilization. Starch retrogradation is a process that occurs when molecules composing gelatinized starch begin to reassociate. Retrogradation is accompanied by increases in turbidity, gel firmness, degree of crystallinity and the appearance of a ' $\mathrm{B}$ ' type $\mathrm{X}$-ray pattern.

The retrogradation of starches from cereal, tuber and legumes has been subjected to detailed studies. However, there is a dearth of information on the influence of physical modification such as defatting and heat-moisture treatment on the rate and extent of retrogradation of starches from different plant origins. Recently, Vasanthan \& Hoover (1992a) and Hoover \& Vasanthan (1994a) have shown that defatting and heat-moisture treatment cause structural changes to occur within the amorphous and crystalline domains of tuber and cereal starches. However, no attempt has been made to explain how these changes influence granule swelling, amylose leaching. granule rigidity and starch chain interaction during gelation and crystallization.

Hydroxypropylation has been shown to reduce starch retrogradation. However, there is a dearth of information on the influence of alkaline conditions used during hydroxypropylation on starch retrogradation. Furthermore, most of the present information on starch retrogradation has come from differential 
scanning calorimetry and $\mathrm{X}$-ray diffraction studies. It is important to measure the retrogradation mechanism of a particular starch using a wide variety of techniques (turbidity development, enzyme digestibility, differential scanning calorimetry, X-ray diffraction and scanning electron microscopy). since one technique alone cannot unravel the different molecular processes occurring during starch gelation and crystallization. Thus, in this study, different physical probes (turbidity development, enzyme digestibility, differential scanning calorimetry. X-ray diffraction and scanning electron microscopy), have been used in an attempt to study how the rate and extent of starch retrogradation is influenced by defatting, heat-moisture treatment, alkali treatment and hydroxypropylation (at different levels of MS).

The hypothesis and objectives underlying this research investigation are outlined below:

Hypothesis 1 : The thermal energy imparted to starch chains during defatting (1propanol water, $\left.3: 1 \mathrm{v} / \mathrm{v}, 7 \mathrm{~h},-82^{\circ} \mathrm{C}\right)$ and heat-moisture treatment $(30 \%$ moisture. $100^{\circ} \mathrm{C}, 16 \mathrm{~h}$ ), and the added moisture content ( $30 \%$ moisture) within starch granules during heat-moisture treatment would increase starch chain mobility, resulting in either increased interaction between amylose - amylose (AM-AM). amylose - amylopectin (AM-AMP), amylopectin - amylopectin (AMP-AMP) chains and/or disruption of starch crystallites. These structural changes would, in turn, radically alter the physicochemical properties of the starch granule. 
Objective 1 : To determine (by physical and chemical methods) the influence of defatting and heat-moisture treatment on starch structure and properties.

Hypothesis 2 : Hydroxypropylation has been shown to occur within the amorphous regions of the starch granule. Therefore, any changes within the amorphous regions during defatting and heat-moisture treatment could either increase or decrease the accessibility of hydroxypropyl groups into the granule interior. Thus, hydroxypropylation at the same level of propylene oxide should result in different levels of molar substitution (MS) in native, defatted and heatmoisture treated starches.

Objective 2 : To determine the degree of accessibility of hydroxypropyl groups into the amorphous regions of potato starch before and after defatting and heatmoisture treatment.

Hypothesis 3 : Changes in starch properties on hydroxypropylation have been attributed solely to the hydroxypropyl group. However, it is also likely, that the alkaline reagents used during hydroxypropylation could also influence starch properties by altering starch chain arrangements within the amorphous and crystalline domains of the starch granule.

Objective 3 : To study the influence of the reagents $\left(\mathrm{NaOH}\right.$ and $\left.\mathrm{Na}_{2} \mathrm{SO}_{4}\right)$ used during hydroxypropylation on the structure and properties of native, defatted and heat-moisture treated starches.

Hypothesis 4: The structural arrangement of amylose and amylopectin chains within the amorphous and crystalline regions of native, defatted and heat- 
moisture treated starches could influence the extent to which hydroxypropyl groups are able to modify starch properties.

Objective 4: To determine the influence of hydroxypropylation at various levels of MS on the thermal and rheological properties of native, defatted and heatmoisture treated starches.

Hypothesis 5 : Changes within the amorphous and crystalline regions of starch granules on defatting, heat-moisture treatment and alkaline treatment could influence the reactivity of the starch granule towards $\alpha$-amylase. Furthermore, steric effects imposed by bulky hydroxypropyl groups could hinder the accessibility of $\alpha$-amylase towards the glycosidic linkages.

Objective 5 : To study the reactivity of $\alpha$-amylase towards native, defatted and heat-moisture treated potato starches before and after hydroxypropylation.

Hypothesis 6 : Starch chains within gelatinized granules and in the continuous medium interact during gel storage. Thus, any change in starch structure on defatting, heat-moisture treatment and alkaline treatment could indirectly influence the rate and extent of starch chain interactions during gel storage. Furthermore, bulky hydroxypropyl groups could hinder this interaction (amyloseamylose [AM-AM], amylose-amylopectin [AM-AP], amylopectin-amylopectin [APAP]) sterically and/or by decreasing the mobility of the interacting chains. 
Objective 6 : To study how the rate and extent of potato starch retrogradation is influenced by defatting, heat-moisture treatment, alkaline treatment and hydroxypropylation (at different MS levels). 


\section{CHAPTER 2}

\section{LITERATURE REVIEW}

\section{1 Starch - Introduction}

Starch is a major reserve polysaccharide of green plants, which is deposited in granular form in seeds, roots, tubers, stems and leaves of plants. The granules are partially crystalline, insoluble in cold water and their size, the shape (Table 2.1), and the composition are essentially genetical. In general, cereal starch granules are small and polyhedric, whereas, tuber starch granules are large, spherical or ellipsoid. In contrast to most starches which have single size distribution of granules, rye, wheat, triticale and barley starches show a bimodal distribution : spherical ' $B$ ' granules $(1-10 \mu)$ and lenticular ' $A$ ' granules $(15-35 \mu)$. The granule size and morphology have received much attention recently, since small granules are important in determining the taste and mouthfeel of some starch based fat mimetics (Alexander, 1992). Pure starch is a mixture of two $\alpha-(1 \rightarrow 4)$ glycosidic bonded glucose polymers, namely essentially linear amylose and branched amylopectin (Table 2.2) in which branching occurs through $\alpha-(1 \rightarrow 6)$ linkages. The ratio of amylose and amylopectin differ depending on the botanical source of starch. The amount of amylose present in starches is in the range of 17 (rice) - 38 (lentil) \%, while amylopectin ranges from 62 to $83 \%$. Granule composition, morphology and supermolecular organization are to a certain extent under genetic control; i.e. they are influenced 
Table 2.1 Size and shape of starch granules from different botanical sources.

\begin{tabular}{|c|c|c|}
\hline Starch source & Shape & Size $(\mu \mathrm{m})$ \\
\hline \multicolumn{3}{|l|}{ Cereal } \\
\hline Wheat & spherical, lenticular & $1-45$ \\
\hline Maize & spherical, polygonal & $2-30$ \\
\hline Waxy maize & polyhedric & $3-26$ \\
\hline Rice & polyhedric & $3-8$ \\
\hline Sorghum & polyhedric, spherical & $4-24$ \\
\hline Barley & lenticular & $2-5$ \\
\hline \multicolumn{3}{|l|}{ Root and tuber } \\
\hline Potato & oval, spherical & $5-100$ \\
\hline Tapioca & truncated, spherical, oval & $4-35$ \\
\hline \multicolumn{3}{|l|}{ Legumes } \\
\hline Smooth pea & reniform (simple) & $5-10$ \\
\hline Wrinkled pea & reniform (compound) & $30-40$ \\
\hline Chick pea & spherical, oval & $8-54$ \\
\hline Cow pea & spherical, oval & $15-85$ \\
\hline Lentil & spherical, oval & $15-36$ \\
\hline Black beans & oval, spherical & $8-34$ \\
\hline
\end{tabular}

Adapted from Swinkels (1985); Blanshard (1987); Hoover \& Sosulski, (1985). 
Table 2.2 Characteristics of amylose and amylopectin

\begin{tabular}{|c|c|c|c|}
\hline \multirow{2}{*}{$\begin{array}{l}\text { Characteristic } \\
\text { Molecular structure }\end{array}$} & \multicolumn{2}{|l|}{ Amylose } & \multirow{2}{*}{$\begin{array}{c}\text { Amylopectin } \\
\text { branched with } \alpha-(1-4) \text { and } \alpha-(1-6) \\
\text { linkages } \\
10^{4}-10^{5}\end{array}$} \\
\hline & $\begin{array}{l}\text { essentially linear with } \\
\text { linkages, slightly branched } \\
700-5000\end{array}$ & $\alpha-(1-4)$ & \\
\hline Average $\mathrm{CL}$ & $100-550$ & & $18-25$ \\
\hline Branch linkage (\%) & $0.2-0.6$ & & $4.0-5.5$ \\
\hline lodine binding $-\lambda \max$ & $640-660 \mathrm{~nm}$ & & $530-570 \mathrm{~nm}$ \\
\hline- color & deep blue & & purple \\
\hline Stability of aqueous solutions & retrogrades & & stable \\
\hline Film properties & strong & & brittle \\
\hline Solubility in water & variable & & soluble \\
\hline \multicolumn{4}{|l|}{ Digestibility } \\
\hline$\alpha$-amylase & $\sim 100$ & & $\sim 90-100$ \\
\hline$\beta$-amylase & $70-96$ & & $50-60$ \\
\hline$\beta$-amylase and debranching enzyme & $\sim 100$ & & $\sim 75$ \\
\hline Gel properties & $\begin{array}{l}\text { stiff, thermally irreversible } \\
\qquad 100^{\circ} \mathrm{C}\end{array}$ & below & soft, thermally reversible below $100^{\circ} \mathrm{C}$ \\
\hline
\end{tabular}


by the biochemistry of the amyloplast (starch synthesizing organelle) as well as by the physiology of the plant (Banks \& Greenwood, 1975; French, 1984; Biliaderis, 1991). However, the exact mechanism by which plant genetic information is translated into a specific granule composition, morphology and organization is unknown. Even the exact role and in vivo regulation of various enzymes involved in starch biosynthesis is still in dispute (Preiss \& Lori, 1980; Robyt, 1984). Only when this information is available it might be possible to manipulate the amount, composition and properties of starch by genetic engineering (Biliaderis, 1991). The fine structure and the molecular order of amylose and amylopectin determine the physicochemical properties of starches.

\subsection{Starch Biosynthesis}

Biosynthesis of starch takes place in a specialized subcellular organelle, the amyloplast, which has a limiting lipoprotein membrane. Figure 2.1 shows the enzymes involved and the metabolic pathways of starch biosynthesis. The enzymes which catalyze the biosynthesis are present within the amyloplast. These enzymes include phosphorylases, starch synthetase and branching and debranching enzymes(Jansson et al., 1995). Sucrose, the starting material of starch synthesis is transported from the photosynthetic tissue to the storage organ. The assembly of starch chains occurs on a lipoprotein matrix. At a certain moment, a minute amount of insoluble polysaccharide deposits and this acts as the nucleus (hilum) of the granule, around which the granule is developed. As 
Fig. 2.1 Schematic representation of the metabolic pathway for starch synthesis (with permission, Jansson et al., 1995). 


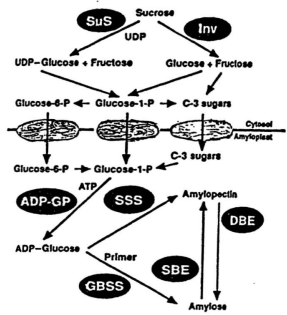

SuS = sucrose synthetase

Inv = sucrose invertase

ADP-GP $=$ ADP-glucose pyrophosphorylase SSS $=$ soluble starch synthase GBSS = granule-bound starch synthase SBE = starch branching enzyme

DBE = debranching enzyme 
the dissolved glucose units are linked to the growing polymer, they simultaneously solidify (Swinkels, 1985). As the granule develops within the amyloplast, it occupies an increasing proportion of the volume, until the internal volume of the amyloplast is completely occupied by starch (Galliard \& Bowler. 1987).

\subsection{Starch production and uses}

Although starch occurs throughout the plant world, there are only a limited number of plants utilized extensively for the production of commercial starches. Sources of commercial starches are maize, wheat, rice, potato, tapioca, arrowroot and sago (Swinkels, 1985). Maize is the major commercial starch produced and in the US, maize accounts for more than $95 \%$ of commercial starches. In addition to maize the US produces limited amount of potato and wheat starches as well. However, in Europe, use of potato in starch production is much greater than that in the US. Tapioca and sago starches are mainly produced in tropical countries such as East Indies and Brazil. Tapioca is imported into the US for use in industrial and food applications and also to produce modified starches (Wurzburg. 1987). In the US, 70\% of the starch is utilized in industrial applications and the remaining $30 \%$ is used in food products. In contrast, European countries use $69 \%$ of starch in food applications and the remaining $31 \%$ is consigned to industrial purposes (Lillford \& Morrison, 1997). 
Starch, in granular form as well as in the paste form has its function in food and non food applications. In food applications, granular starch is utilized as a dusting agent for candy and carrying agent for baking powder. Small granular starches $(<2 \mu$ in diameter) are proposed for fat mimetics. A mixture of small particles dispersed in a starch gel matrix resembles the texture of butter in which fat micelles are dispersed in a liquid fat matrix (Jane, 1997a). In non food uses, granular starch is utilized as a dusting agent in pharmaceuticals (tablets). antiperspirant, and in facial powder substitutes (oat, maize). Granular starch has been used in various products depending on their size and shape. The large granular wheat starch has been shown desirable as "silt material" for coating on carbonless copy paper. Spherical aggregates of small granular starch are used as flavour carriers.

In the paste form, starch is used in food products as a thickener in semi solid foods, pie fillings, sauces, oil mimetics and to provide texture (body) to beer and soft drinks (Jane, 1997a). In non food applications, starch paste has its functions as a coating agent in pharmaceuticals, encapsulating agent in agrochemicals (pesticides), textiles, adhesive, paper and board industry. Starch based products are being considered for use in surfactants (detergents), bleaching boosters (bleach under low temperature) and degradable plastics (Entwistle et al., 1998).

In these applications, the ability of starch to produce a viscous paste when heated in water is its most important property. In addition, the appropriate paste 
quality with respect to clarity. stability towards heat, shear, $\mathrm{pH}$ and resistance to syneresis may also be important depending on the application.

\subsection{Structure of starch}

\subsubsection{Major components}

\subsubsection{Amylose}

\subsection{Structure}

Amylose is found with a molecular weight ranging from $1 \times 10^{5}$ to $2 \times 10^{6}$ $\mathrm{g} /$ mole and the number of glucose residues per molecule, degree of polymerization (DP), ranging from 930-4920 (Table 2.3). Although considered to be essentially linear, amylose is not completely hydrolyzed by $\beta$-amylase (Hizukuri et al., 1981). Greenwood \& Thompson (1962) reported that $\beta$ amylolysis limits of amylose extracted from various starches range from $72-95 \%$ and some of the limits are presented in Table 2.3 The incomplete $\beta$-amylase hydrolysis indicates, that a certain degree of branching is present in amylose. According to Hizukuri et al. (1981), the branching occurs through $\alpha-(1 \rightarrow 6)$ links and the amount of branching depends on the origin of amylose and is in the range of $\mathbf{2 5 - 5 5 \%}$ on a molar basis. The average number of branch linkages per branch molecule is $4-18$, whereas the percentage of branch linkages is 0.27 $0.68 \%$. The branch chains are usually moderately long, but a few may be as small as glucosyl to maltotetraosyl $\left(G_{1}-G_{4}\right)$. Amylose leached from granules immersed in water just above their gelatinization temperature has a lower 


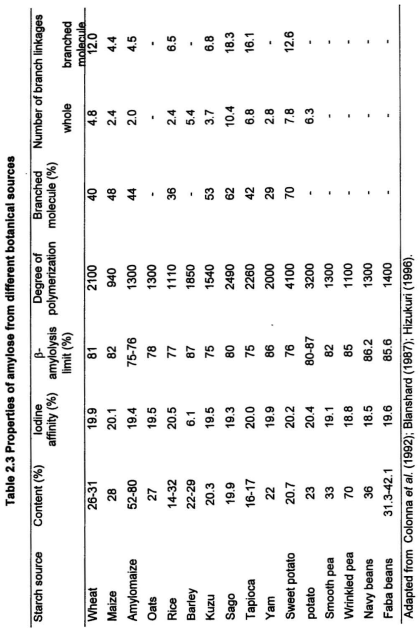


molecular weight and higher $\beta$-amylolysis limit (90-100\%), than more extensively branched amylose which is leached at higher temperatures ( $\beta$-amylolysis limit 70-80\%) [Banks \& Greenwood, 1975; Takeda et al., 1986]. Despite the slight branching, the branched amylose appears to behave like a linear polymer forming films and helical inclusion complexes with ligands. Amylose of some starches has been shown to contain phosphate groups [maize $0.02-0.03 \%$, potato $0.04-0.13 \%$ of dry starch (Galliard \& Bowler, 1987)] probably attached to C-6 of glucose residue (Banks \& Greenwood, 1975).

\subsection{Conformation}

It has been postulated that the conformation of amylose is slightly helical due to the natural twist present in the chair conformation of glucose (Kowblansky, 1985). Amylose in solid state shows two polymorphs; 'A' (Fig. 2.2a) and 'B' (Fig. 2.2b) [Wu \& Sarko, 1978a], which give similar X-ray patterns as the amylopectin crystallites in native starches. Imberty et al. $(1987 ; 1988)$ have shown that ' $A$ ' and ' $B$ ' polymorphs are right handed sixfold double helices. However, conflicting hypothesis still exists concerning the molecular conformation of amylose in aqueous solution (Banks \& Greenwood, 1971; Senior \& Hamori, 1973; Cheetham \& Tao, 1997). Banks \& Greenwood (1971) proposed three model conformations for amylose in aqueous solution (Fig $2.3 \mathrm{a}-\mathrm{C}$ ). In both 
Fig. $2.2{ }^{13} \mathrm{C}$ CP/MAS NMR spectra of highly crystalline (A) 'A' type amylose, (B) 'B' type amylose (adapted from Horii et al., 1987). 


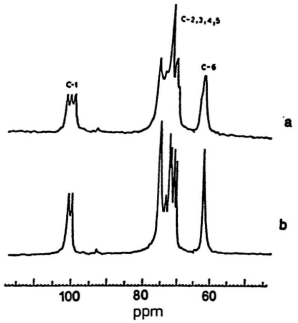


Fig. 2.3 Models proposed for the amylose in aqueous solution (with permission, Banks \& Greenwood, 1971).
A) random helical coil (6 glucose units per turn).
B) interrupted helix.
C) random coil with no helical character. 

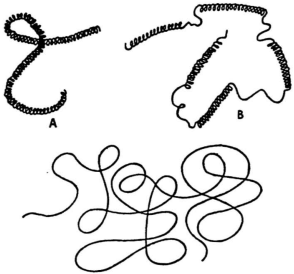

C 
random helical coil (Fig. 2.3a) and interrupted helix (Fig 2.3b), the helical portions are thought to be stabilized by intramolecular hydrogen bonds. The random coil conformation (Fig 2.3c) exists in neutral aqueous solutions as well as in solvents such as dimethyl sulphoxide (DMSO), whereas the helical conformation is attained in neutral or alkaline solutions in the presence of complexing agents (lipids, iodine) [Banks \& Greenwood, 1971]. Senior \& Hamori (1973) suggested that amylose conformation shows regions of loose and extended helices which alternate with shorter random coll segments. When amylose forms a complex, the loose helix is forced into a tighter helical conformation by the complexing agent. In contrast to Banks \& Greenwood (1971), Cheetham \& Tao (1997) have shown [using the changes in chemical shifts of ${ }^{13} \mathrm{C}$ NMR (nuclear magnetic resonance), optical rotation and limiting viscosity measurements] that amylose conformation in $100 \%$ DMSO is helical rather than a random coil. With the addition of water, the intramolecular hydrogen bonding in amylose (when amylose is in DMSO) is gradually replaced by amylose - water intermolecular hydrogen bonds (amylose / DMSO / water), leading to conformational changes. When the concentration of DMSO is decreased from $100 \%$ to $66.6 \%$, amylose conformation changes from tight helix $\rightarrow$ loose helix. Further decrease in DMSO (66.6 to $33.3 \%$ ) causes conformational change from loose helix $\rightarrow$ random coil [Cheetham \& Tao, $1997]$. 
Amylose in solution presents two features which are important in the functionality of starch based products; namely the ability to form inclusion complexes with desirable ligands and the ability to form interchain associations in polysaccharide chains leading to gelation or precipitation.

Amylose combines with a variety of compounds like iodine, aliphatic alcohols, aliphatic hydrocarbons, fatty acids and monoacylglycerol (MG) to form so-called inclusion complexes (Fig. 2.4) which are insoluble at room temperature (Teitelbaum et al., 1978; Carlson et al., 1979; Swinkles, 1985). Inclusion complexes are not formed as a result of a chemical reaction, but have been defined as addition compounds (complexes) in which 'guest' entity fits into and is surrounded by the lattice of the 'host' molecule. The bonds involved in inclusion complex are Van der Waals attractive forces which are quite weak but sufficient to provide the formation of stable complexes (Osman-Ismail, 1972). In the helical form, the interior of the helix is built up by $\mathrm{C}-\mathrm{H}$ groups and glycosidic oxygen atoms forming a lipophilic core, while the polar hydroxyl groups are positioned on the outer surface of the helix (Banks \& Greenwood, 1971). Carlson et al. (1979) have shown that the helical space in $\mathrm{V}$ amylose conformation is too narrow to accommodate the bulky polar group of the lipid molecule. Hence, the polar group must exist outside the amylose helix.

lodine, in the form of polyiodide ions (up to $r_{13}$. but mainly as $r_{3}$ or $r_{5}$ ). can bind with amylose (Teitelbaum et al., 1978). The complex produces a deep blue colour which is used to identify amylose containing starches and to measure 
Fig. 2.4 Schematic illustration of amylose - lipid complex (adapted from Carlson et al., 1979). 
Polar group of llpid molecule

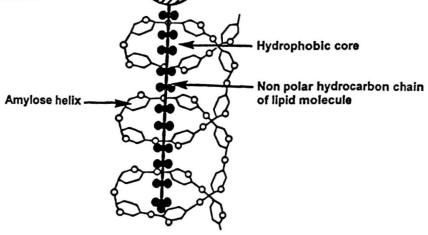


Fig. $2.5 \mathrm{~V}$ X-ray diffraction pattern of amylose - lipid complex showing characteristic $d$ - spacings (4.4-4.3, 6.5-6.8, 11.3-12). (adapted from Zobel, 1988a). 


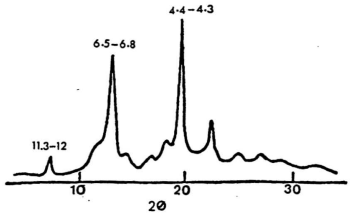




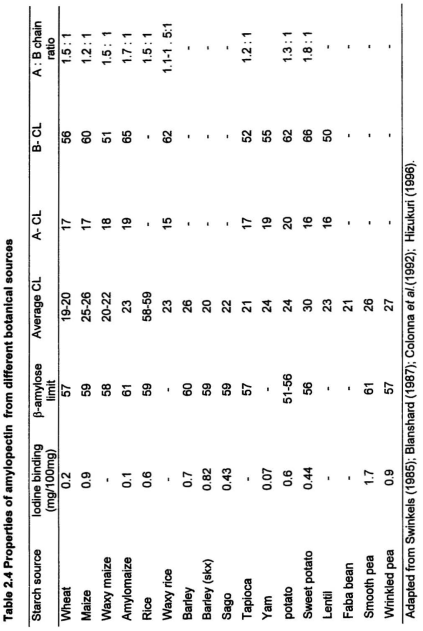

ลี 
inner and outer chain lengths (Robin et al., 1974; Hizukuri, 1986). Although the main structural features of amylopectin have been known for some 50 years, details of the fine structure are still lacking. There is still uncertainty about the detailed arrangements of the constituent linear chains of (1-4) linked $\alpha$-glucose residues.

Investigation of amylopectin structure is a case in point of where progress is dependent on methodology development (both enzymatic and instrumental methods). Hydrolysis of amylopectin with a debranching enzyme and separation of the digest (containing linear chains) using exclusion gel filtration technique normally gives a bi- or trimodal elution profile, indicating the presence of chains with different chain length or molecular weight (Hizukuri, 1986).

A number of models have been proposed for the amylopectin molecule. including comb-like model and laminated structure (Staudinger \& Husemann, 1937). However, currently accepted structural models are those derived from the cluster models of Nikuni (1969) and French (1972) [Fig. 2.6a]. These models exhibited the presence of chain segments which are designated as 'A', 'B' and ' $C$ '. The ' $A$ 'chains are joined to the remainder of the molecule with a single 1,6 bond, ' $\mathrm{B}$ ' chains are joined through a 1,6 bond but may carry one or more 'A' and/or ' $\mathrm{B}$ ' chains on primary hydroxyl groups; ' $\mathrm{C}$ ' chain carries the sole reducing group (Zobel, 1988b).

A similar model (Fig 2.6b) was proposed by Robin et al. (1974), from data derived from sequential treatment of potato amylopectin with debranching 
Fig. 2.6 Models proposed for amylopectin (with permission).
A) Cluster model of French (1972).
B) Robin et al. (1974).
C) Manners \& Matheson (1981). 

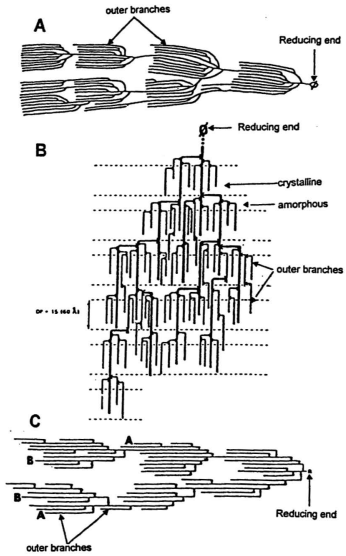
enzymes and $\beta$-amylase, its $\beta$-limit dextrin and a derived acid-resistant amylodextrin. The significance of this model was the presence of three chain populations with $\mathrm{CL}$ of $15-20,45$ and 60 . The chains with $\mathrm{CL}$ of $15-20$ are in highly ordered clusters, which are linked to each other by much longer chains, which would correspond to the longer ' $\mathrm{B}$ ' chains ( $C L \mathrm{45}$ ) found in elution profiles.

Based on the polymodal distribution of chain profile (Fig. 2.7a,b) elucidated from gel permeation HPLC (high performance liquid chromatography) of amylopectin. Hizukuri (1986) proposed a refined cluster model (Fig. 2.7c). 'A' chains are represented by fraction A which eluted last while various B chains are represented by fractions ' $\mathrm{B}_{1}-\mathrm{B}_{4}$ '. Moreover, ' $\mathrm{A}$ ' and ' $\mathrm{B}_{1}$ ' makes a single cluster. Chains in fraction ' $B_{2}$ ' extend into two clusters, those in fraction ' $B_{3}$ ' extend into three clusters, and the chains in fraction ' $\mathrm{B}_{4}$ ' extend into more than four clusters (Fig. 2.7a,b). Hizukuri (1986) showed that amylopectins isolated from potato, tapioca, kudzu and waxy rice the average $C L$ of the fraction ' $\mathrm{B}_{1}$ ', ' $\mathrm{B}_{2}$ ' and ' $\mathrm{B}_{3}$ ' were $20-24,42-48$ and $69-75$ respectively, the relative lengths being $\sim 1: 2: 3$. The $\mathrm{CL}$ of ' $\mathrm{A}$ ' chains are in the range of 12-16 which was in agreement with the exterior $\mathrm{CL}$ of amylopectin as reported by Manners (1985). Similar CL values have been reported by French (1972), Robin et al. (1974) and Hood \& Mercier (1978) and the values were 12,15 and 15 , respectively. The sum of ' $A$ ' and ' $B_{1}$ ' fractions represents $80-90 \%$ of total chains and constitute a single cluster and the 10-20\% are in inter-cluster connections (Hizurkri, 1986). 
Fig. 2.7 Chain distribution of (A) potato, (B) waxy rice and (C) the proposed model for amylopectin (adapted from Hizukuri, 1986). 


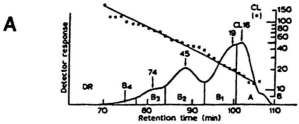

B
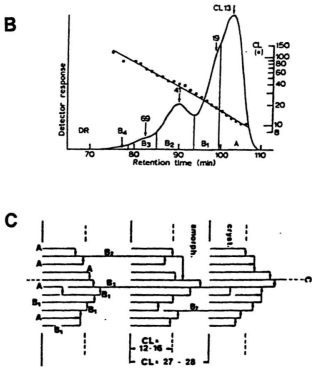
The ratio of ' $A$ ' to ' $B$ ' chains (2:1) was first reported by Marshall \&. Whelan (1974). However, a critical examination by Manners \& Matheson (1981) revealed that the 'A' to 'B' chain ratio was about 1:1. Hizukuri (1986) reported that the $A$ : B ratios are 0.8-0.9:1 for tapioca, kuzu and potato, and 2.2:1 for waxy rice amylopectin.

The cluster model is in accordance with the relatively high viscosity of amylopectin, the crystallinity of the macromolecule as revealed by $\mathrm{X}$-ray analysis and the relative resistance of parts of the molecule to attack by acid and amylolytic enzymes (Manners, 1985). All these structures have the concept that the branch points are arranged in clusters of short chains.

On the basis of the structures of Naegeli dextrins (obtained by high performance anion exchange chromatography) and data obtained from other studies (Jenkins et al, 1993; Jenkins \& Donalds, 1995; Jane et al,, 1997b), Jane (1997b) proposed two structurally different models of ' $A$ ' and ' $\mathrm{B}$ ' type amylopectin (Fig. 2.8). The structures show that $\alpha-(1 \rightarrow 6)$ branch linkages of 'A' starches are more scattered. Substantial amounts of branch linkages are located within the crystalline regions (which are protected from acid hydrolysis), whereas the branch. linkages in the amorphous region are hydrolysed during acid treatment. The branch linkages in the ' $\mathrm{B}$ ' type amylopectin are clustered in the amorphous region and are susceptible to acid hydrolysis. These models show that the repeating distance of ' $A$ ' and ' $B$ ' type amylopectin are 9.0 and $9.2 \mathrm{~nm}$ (Fig. 2.8), respectively (Jane, 1997b). 
Fig. 2.8 Proposed models for 'A' (from waxy maize) and 'B' (from potato) type amylopectin branching patterns. $\mathrm{A}$ and $\mathrm{C}$ represent amorphous and crystalline regions, respectively (adapted from Jane, 1997b). 
A- type amylopectin

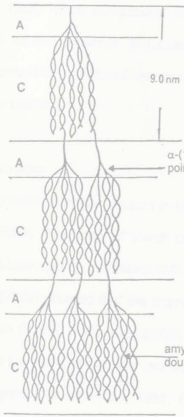

A - amorphous
B- type amylopectin

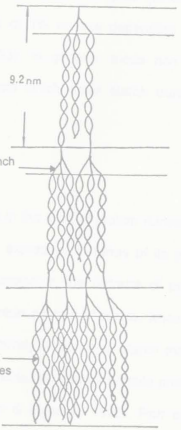

C - crystalline 


\subsubsection{Minor components of starch}

The minor components of starch exist either as surface materials on the granules or as internal components within the granule matrix. The most important minor components of starch are proteins and lipids (Lineback \& Rasper, 1988) which are present at levels of $1 \%$ or less depending on the botanical source (Eliasson \& Larsson, 1993). In general, these non starch components are considered as contaminants which enter starch during the extraction process.

\subsubsection{Proteins}

Generally, nitrogen present in starch is in the form of protein (Lineback \& Rasper, 1988). The purity of starch can be expressed in terms of its protein content. Starch purity increases with a decrease in the amount of protein. Swinkels (1985) showed that the average protein content of maize, wheat and potato were $0.35 \%, 0.4 \%$ and $0.06 \%$ respectively. Proteins in starch may be present in the form of granule surface proteins, as internal granu'. roteins, or as enzymes (Lowry ot al., 1981; Eliasson \& Larsson, 1993). Part of the nitrogen may also be present in association with starch lipids (e.g. lysophosphatidylcholine in wheat starch). Lowry et al. (1981) have shown that the protein content of well washed pure ' $A$ ' wheat starch was $0.1 \%$, while $\sim 10 \%$ of the protein was associated with the granule surface. Proteins which are associated with the surface of the granule can be readily extracted with dilute 
salt under mild conditions that cause no disruption of granules. The internal proteins are not released by dissociating agents (e.g. sodium dodecyl sulphate [SDS]) until the granules have been gelatinized by heating. The requirement for disruptive conditions to release these proteins indicates, that they are buried within the matrix of the granules. The sub-units of internal proteins obtained on sodium dodecyl sulphate - polyacrylamide gel electrophoresis are of higher molecular weight than the surface proteins (Lowry ef al., 1981). It is likely that the

internal proteins may represent residual material from lipoprotein membranes of the original amyloplasts or of membrane bound starch synthesizing systems employed during development (Galliard, 1983).

\subsubsection{Lipids}

Lipids associated with isolated cereal starch granules have been found to occur on the surface as well as inside the granule (Morrison, 1981). The surface lipids are mainly triacylglycerol, followed by free fatty acids, glycolipids and phospholipids and they include those that may have been present on the granule surface in situ in the plant tissue as well as the non starch lipids, which are absorbed into the surface layer of starch granule during isolation (Morrison, 1981; Galliard \& Bowler, 1987; Vasanthan \& Hoover, 1992b). Non-starch lipids occur as spherosomes, concentrated in the sub-aleurone region, and also as components of membranes and organelles associated with storage protein (Morrison, 1981), Since it is not possible to distinguish between these two types 
of surface lipids on the basis of solvent extraction techniques, it has been suggested that all lipids found on the surface have to be considered as starch lipids (Galliard \& Bowler, 1987). The internal lipids of cereal starches are predominantly monoacyl lipids, with the major component being lysophospholipids (of which lysophosphatidylcholine is the major component) and free fatty acids (Hargin \& Morrison, 1980; Morrison, 1981). It is likely that both surface and internal lipids may be present in the free state as well as bound to starch components, either in the form of amylose inclusion complexes or linked via ionic or hydrogen bonding to hydroxyl groups of the starch components. Free lipids are easily extractable by solvent systems at ambient temperatures (Morrison, 1981), whereas prolonged extraction with hot aqueous alcoholic solvent systems (Morrison, 1981) or disruption of the granular structure by acid hydrolysis (Goshima et al., 1985) is required for the efficient removal of bound lipids. The amount of total starch lipids (surface and bound) has been found to be in the range of $0.7-1.2 \%$ in cereals (Morrison \& Milligan, 1982; Vasanthan \& Hoover, 1992b; Takahashi \& Seib, (1988), 0.01-0.87\% in legumes (Hoover \& Sosulski, 1985), and $0.08-0.19 \%$ in tubers and roots (Vasanthan \& Hoover, 1992b; Emiola \& Delarosa, 1981; Goshima et al., 1985).

Zobel (1988a) suggested that lipids in starches may be responsible for effecting an amylose separation within the granule. This would imply that the starch polymers of low lipid containing starches (potato, lentil, cassava) may be more associated with each other in the native granule than those of high lipid 
containing starches (wheat, corn). Morrison \& Laignelet (1983) showed that the presence of lipid decreases the iodine binding capacity of cereal starches by 20 $30 \%$ which may be interpreted as the proportion of amylose complexed with lipid and hence unavailable to complex with iodine.

\subsection{Amylose-lipid inclusion complex}

Amylose - lipid inclusion complexes (Fig 2.4) have been shown to influence the texture and the structural stability of cereals and starch based products (Lund, 1984). These complexes are effective in decreasing the rate of bread staling (Krog \& Jensen, 1970), improving the texture in extruded starch containing products (Mercier et al., 1980), improving structural integrity in cereal kernels (parboiled rice) during cooking (Biliaderis et al., 1993) and preventing stickiness in mashed potato granules (Hoover \& Hadziyev, 1981).

\subsection{Occurrence of amylose-lipid complex}

The existence of naturally occurring amylose-lipid complexes in starch granules has been the subject of much controversy. For many years, it has been observed that native starches with $<30 \%$ amylose do not exhibit the naturally occurring 'V' X-ray pattern (Galliard \& Bowler, 1987) except for wrinkled pea, amylomaize and some other maize genotypes [sugary (su), and dull (du) (Gernat et al., 1993)]. The absence of a ' $\mathrm{V}$ X- ray pattern does not necessarily indicate the lack of amylose-lipid complexes in native starches; it suggests that the 
helices are not organized in a three dimensional array. Recently ${ }^{13} \mathrm{C}$ CP/MAS NMR (cross polarization magic angle spinning nuclear magnetic resonance) studies provided the evidence for the presence of amylose-lipid complex in granules of native oat, maize rice, barley and wheat starches (Morrison et al., 1993a, 1993b; Morgan et al., 1995). The spectral features (Fig. 2.9) of cereal starches indicative of the single ' $\mathrm{V}$ amylose helix were : 1) the signal of $\mathrm{C}-1$ at 103-104 ppm representing ' $\mathrm{V}$ form, and 2) the broad peak at $31 \mathrm{ppm}$ (represent the mid-chain methylene carbons of monoacyl lipids) which reflects a near-solid state structure of lipids due to steric constrains in the helical cavity. Morrison et al. (1993a, 1993b) showed that amylose exist partially as lipid-complexed amylose (L-AM) with a lysophospholipid to L-AM ratio of 1: 7 and partially as lipid-free amylose (F-AM). Table 2.5 shows different barley starches with their LAM and F-AM contents. Waxy barley starches used in the study contained 0.8$4.0 \%$ L-AM and $0.9-6.45 \%$ F-AM, whereas in normal barley starches corresponding values were $6.1-7.2 \%$ and $23.1-25.0 \%$, respectively. Gernat et al. (1993) have also shown the existence of amylose - lipid complexes in native starch granules by $\mathrm{X}$-ray diffraction studies of enzymatically degraded wheat starch.

The ability of lipids to form complexes depends on the type of lipid. Using the iodine binding capacity of starch, it has been shown that saturated MG are more effective in complex formation (Lagendilik \& Pennings, 1970; Krog. 1971) than unsaturated MG (Eliasson \& Larsson, 1993). The amount of fatty acid 
Fig. $2.9{ }^{13} \mathrm{C}$ CP/MAS NMR spectra of $\mathrm{V}$ amylose in waxy barley starch (Chalky Glen), with inset of the $10-50 \mathrm{ppm}$ region at $\times 20$ scale expansion. The marked resonance is for midchain methylene carbons of fatty acids in lysophospholipid with a chemical shift of $31.2 \mathrm{ppm}$ (A). Resonance from polysaccharide carbons (B-E) are B = C-6 (61.3 ppm), C = unresolved C-2, C-3, C-5 (71.4 ppm), D = C-4 (80.9 ppm), E = C-1 (100.5 ppm) with some helical amylose (103 ppm) [With permission, Morrison et al., 1993b]. 


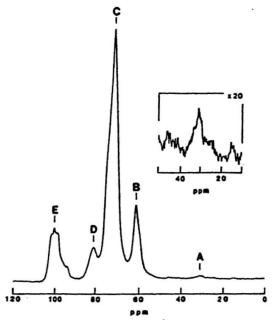


Table 2.5 Lipid complexed amylose (L-AM) and lipid-free amylose (F-AM) in waxy and normal barley starches

\begin{tabular}{|c|c|c|}
\hline Variety & L.AM (\%) & F.AM(\%) \\
\hline \multicolumn{3}{|l|}{ waxy } \\
\hline Summier Mochi & 0.8 & 0.9 \\
\hline Dango Mugi & 1.2 & 0.9 \\
\hline Masan Naked & 1.5 & 0.9 \\
\hline Tokushima Mochimugi (a) & 1.7 & 1.4 \\
\hline Tokushima Mochimugi (b) & 2.1 & 1.5 \\
\hline Chalbori & 1.6 & 1.8 \\
\hline lyatomi Mochi & 2.1 & 1.8 \\
\hline Waxy Oderbrucker & 2.8 & 2.4 \\
\hline Bozu Mochi & 3.2 & 2.2 \\
\hline Wapana & 3.4 & 3.1 \\
\hline Wanupana & 3.5 & 3.0 \\
\hline Washonupana & 4.0 & 3.4 \\
\hline \multicolumn{3}{|l|}{ Normal (non waxy) } \\
\hline Ckalky Glen & 6.1 & 23.1 \\
\hline Midas & 6.2 & 24.0 \\
\hline Hector & 5.4 & 25.0 \\
\hline Shopana & 7.0 & 23.5 \\
\hline Compana & 7.2 & 23.3 \\
\hline Glen & 6.8 & 25.9 \\
\hline
\end{tabular}

Adapted from Morrison et al. (1993b). 
bound by amylose increases with an increase in the chain length of the fatty acid. Furthermore, the increase in unsaturation decreases the ability of complex formation (Hahn \& Hood, 1987), possibly due to the fact that saturated fatty acids with straight hydrocarbon chains may fit into the amylose helical cavity more easily than unsaturated fatty acid hydrocarbon chains which may be bent at various angles. Moreover, unsaturated fatty acids have greater solubility in water and may therefore, exist in the free state in higher amounts than saturated fatty acids which are less soluble (Hahn \& Hood, 1987).

The acyl chain of amylose-lipid complex is considered as a straight "rodlike* structure. In linoleic acid, approximately $50 \%$ of fatty acid chains are of cis9, cis-12, $18: 2$. Therefore, it might be expected that the kinks introduced by two cis-double bonds would interfere with complex formation. Galliard (1983) showed that there is evidence to show the complex formation between amylose and linoleic acid is slightly less stable than that between amylose and the corresponding fully saturated fatty acid, the free energies determining the conformation of the complexes with saturated or unsaturated fatty acids are presumably relatively similar. Furthermore, Riisom et al. (1984) have shown that cis unsaturated compounds were more effective than trans unsaturated monoelaidate and the saturated monopalmitate in complex formation. However, surprisingly, naturally occurring amylose-lipid complexes in starch consist of Iysolecithin which is rich in cis-cis linoleic acid. 
Formation of ' $\mathrm{V}$ amylose has been observed during swelling and gelatinization of starch granules (Morrison \& Milligan, 1982; Eliasson \& Larsson. 1993) on heat-moisture treatment (Zobel, 1988a), on extrusion cooking of lipid containing cereal starches (Mercier et al., 1980), and after addition of monoacyl lipids to starch under appropriate conditions (Hoover \& Hadziyev, 1981; Biliaderis et al., 1986a). The hydrothermal conditions induce the mobility of amylose chains to complex with naturally occurring or added monoacyl lipids, leading to the formation of larger assemblies detectable by $\mathrm{X}$-ray diffraction. Extrusion of cassava starches (Mercier et al., 1980) with 2-4\% monoacyl lipids at $22 \%$ moisture, showed formation of two types of structures depending on the extrusion temperature. When starches were extruded below $170^{\circ} \mathrm{C}$, they showed $\mathrm{X}$-ray pattern termed 'hydrated - V' pattern [d spacings (the distance between the planes in the crystal) at $4.4,6.8$ and $12.0 \AA \mathrm{A}]$, whereas when the extrusion temperature was above $185^{\circ} \mathrm{C}$ and the moisture content was $<13 \%$, the 'extruded' type X-ray pattern was observed. Extruded type was characterized by the slight displacement of diffraction peaks to a lower angle (e.g. the peak at $9^{\circ} 54^{\prime}$ in hydrated ' $V$ 'type appears at $9^{\circ} 03^{\prime}$ in 'extruded' type).

X-ray, DSC (differential scanning calorimetry) and structural analysis data (Biliaderis \& seneviratne, 1990; Biliaderis \& Galloway, 1989) have shown that the amylose-lipid complex in the solid state shows the existence of two structurally different forms; form I and form II (Fig. 2.10), based on the crystallization conditions (temperature, type of ligand, etc.). Form I is obtained under conditions 
Fig. 2.10 A typical DSC thermal curve ( $50 \%$ solid) of a cereal starch showing the different melting transitions and the corresponding structural domains undergoing a phase change.

$M_{1} \& M_{2}$ : melting of amylopectin crystallites at intermediate moisture content. $\mathrm{M}_{3}$ : melting of Form I amylose - lipid complex

$M_{4}$ : melting of Form II amylose - lipid complex (with permission, Biliaderis \& Galloway, 1989; Biliaderis \& Seneviratne, 1990). 
favouring rapid nucleation. This gives an amorphous $X$-ray pattern showing the lack of properly packed ordered systems, whereas form II shows the typical 'V pattern, reflecting the well developed long range order (Biliaderis \& Galloway, 1989).

\subsection{Stability of amylose-lipid complex}

Starch - lipid complex formation takes place when both amylose and lipid are in solution. Therefore, in order to form complexes, lipids must be in a suitable dispersed state. The optimal conditions for amylose - lipid complex formation are directly related to the lipid monomer concentration (Larsson, 1983). The most effective state of lipid in complex formation is the micellar solution (Fig. 2.11a). since its monomer concentration in equilibrium with a micellar solution is high. Furthermore, lipids in the lamellar phase (Fig. 2.11a,b) are excellent in complex formation (Riisom ot al., 1984) because of their ability to form fine dispersions (Larsson, 1983).

\subsection{Thermal and rheological properties of amylose-lipid complex}

Amylose-lipid complex has been shown to alter gelatinization parameters. granule swelling, solubility, amylose leaching and viscosity of starches. In general, formation of amylose-lipid complex decreases gelatinization enthalpy. granule swelling, solubility and amylose leaching of starch. 
Fig. 2.11 Structures in binary lipid-water systems.

A) Binary lipid-water system characteristic of polar lipids which form micellar solutions. The horizontal axis defines the composition and the vertical axis the temperature. Lipid molecules are illustrated by the polar head (a circle) and one attached chain tail (adapted from Larsson, 1983).

B) Structure of a fragment of a particle of the lamellar phase dispersed in water (adapted from Larsson \& Dejmek, 1990). 
DSC studies show that, the melting transition of amylose-lipid complex occurs in the temperature range of $85-130^{\circ} \mathrm{C}$. In Figure $2.10, \mathrm{M}_{3}$ and $\mathrm{M}_{4}$ transitions show the melting of form I (with low melting temperature) and form II (with high melting temperature) complexes respectively. The melting temperature of the complex is influenced by hydrocarbon chain length (Eliasson \& Krog. 1985; Biliaderis \& Galloway, 1989), complex concentration (Biliaderis et al., 1985), crystallization temperature (Biliaderis \& Galloway, 1989) and moisture content (Biliaderis et al., 1985). Eliasson \& Krog (1985) have reported that melting temperature of potato amylose-lipid complex increased when the chain length of monoacyl lipid increased from $\mathrm{C}_{12}$ to $\mathrm{C}_{18}$. Furthermore, Biliaderis et al. (1985) have shown that peak melting temperature of amylose-monopalmitin. amylose-lysolecithin and amylose-lauric acid complexes increased with increase in complex concentration.

Influence of moisture content on amylose - lipid complex formation showed that at moisture contents $>80 \%$, a single endotherm was observed for melting of amylose-lipid complex, whereas for the moisture contents $<50 \%$, melting was shown by two endotherms which were separated by an exothermic peak. Such nonequilibrium melting is due to partial melting followed by recrystallization and final melting of the complex (Biliaderis et al., 1985).

Lonkhuysen \& Blaknestijn (1976) reported that the stability of swollen granules was greatly enhanced when MG was added to the starch after gelatinization, but the swelling power was not affected. Hoover \& Hadziyev 
(1981) reported that, swelling power and solubility of potato starch decreased when the starch was complexed with saturated 1-monoacylglycerols. Solubility decreased by $8 \%$ with $\mathrm{C}_{8}$ and $90 \%$ by $\mathrm{C}_{14}$. Swelling power dropped steadily when the MG chain length increased from $C_{8}$ to $C_{14}$ (Hoover \& Hadziyev, 1981).

Evans (1986) showed that viscosity of heated waxy maize starch increased in the presence of SDS or cetyltrimethylammonium bromide (CTAB). Eliasson et al. (1988) reported that the addition of lipids (CTAB, saturated MG) into normal maize, cross-linked waxy maize and acetylated high amylose maize increased the dynamic viscosity of starches. The above authors suggested that the additives with two (lecithin) or three (soybean oil) hydrocarbon chains affect the viscoelastic properties of maize starch independent of amylose content.

\subsubsection{Super molecular order of the starch granule}

The super molecular order of starch granule (the organization of amylose and amylopectin within the granule) which governs most of the physicochemical properties of starch, is an important aspect of starch structure determination. The structure of starch has been subjected to many investigations and much speculation, nevertheless, the detailed arrangement of amylose and amylopectin within the starch granule is still under investigation. Different techniques have been employed to study the structural organization of starch granules. Quantitative structural analysis has been previously relied on a combination of enzymatic and chemical methods. Transmission and scanning electron 
microscopy as well as small and wide angle $\mathrm{X}$-ray diffraction have highly contributed to the structure determination. Today, high resolution nuclear magnetic resonance in the form of ${ }^{1} \mathrm{H}$ NMR, ${ }^{13} \mathrm{C}$ NMR and ${ }^{13} \mathrm{C}$ CPMMAS NMR have proven to be more effective, non invasive quantitative analytical tool.

The growth of starch granule by concentric deposition of layers has been known for many long years. Nikuni (1978) proposed a model which incorporated the amylose and amylopectin components including the appearance of concentric rings. Lineback (1984) proposed a modified version (Fig. 2.12) of Nikuni's (1978) model, which incorporated the cluster model of amylopectin. The current models (Fig. 2.13a) of granule are based on the fact that the crystalline structure of granule consists of radially arranged amylopectin clusters (Jenkins et al., 1993). However, the exact arrangement of starch chains within the granule remains unclear.

It is now accepted that the starch granule is composed of both crystalline and amorphous regions. The fact that the crystalline component primarily consists of amylopectin was affirmed by the crystallinity, shown by granules after amylose was leached out, and waxy maize starch with no amylose being semi crystalline (Zobel, 1988b). The crystalline nature of native starch granules display a "Maltese cross" when viewed under polarized light. The positive birefringence indicates that there is a high degree of molecular orientation in the granule (Imberty \& Perez, 1996). Electron or optical microscopy has 
Fig. 2.12 Schematic model of starch granule proposed by Lineback (with permission, Lineback, 1984) 


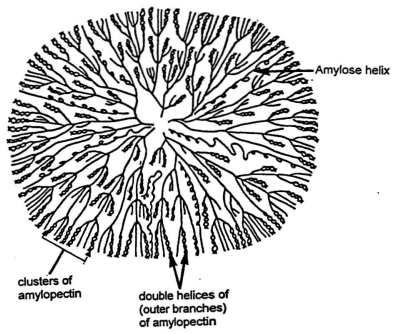


Fig. 2.13 Inner structure of starch granule showing crystalline and amorphous regions.

(A) stacks of semi crystalline lamella are separated by amorphous growth rings.

(B) magnified view of a stack made up of alternating crystalline and amorphous regions.

(C) the crystalline lamellae consist of double helices made up of amylopectin branches (adapted from Donald et al., 1997). 
confirmed the presence of well defined lamella which are also known as growth rings (Fig. 2.13a), especially in the granules treated with acid or amylolytic enzymes (Jenkins et al., 1993; Eliasson \& Larsson, 1993). These rings are alternately semi crystalline and amorphous and are generally $120-400 \mathrm{~nm}$ in size. These semi crystalline growth rings are composed of stacks (Fig, 2.13b) of alternating crystalline [double helices of short DP chains of amylopectin (Fig. 2.13c)] and amorphous [amylopectin branch points (Fig. 2.13c)] lamella [Kassenbeck, 1978]. This represents an average cluster of amylopectin (Fig. $2.13 \mathrm{c}$ ) which is described and measured as repeat distance or periodicity.

Electron microscopy has shown that there is a periodicity of $6-7 \mathrm{~nm}$ along a radially oriented molecular axis (Kassenbeck, 1978; Yamaguchi et al., 1979). This value is smaller than the value deduced $(9-10 \mathrm{~nm})$ from small angle neutron scattering (Blanshard et al, 1984) and small-angle X-ray scattering (Oostergetel \& Van Bruggen, 1989) studies of various starches. However, X-ray diffraction profile analysis showed that this periodicity is independent of the botanical source and the size is $9 \mathrm{~nm}$ for all the studied starches (Jenkins et al., 1993; Jenkins \& Donald, 1995). Oostergetel \& Van Bruggen (1993) proposed a superhelical lamellar structure (Fig. 2.14) for potato amylopectin, using electron optical tomography and cryoelectron diffraction data from non disrupted granule fragments: The linear segments of double helices (Fig. 2.14a) were crystallized into lamella of $5 \mathrm{~nm}$ alternating with amorphous layers (Fig. 2.14b). The neighboring helices interpenetrate each other forming a continuous 


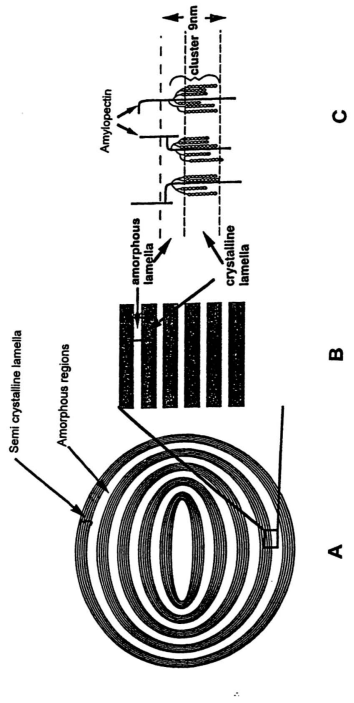


Fig. 2.14 Schematic model for the arrangement of amylopectin (super helical structure) in potato starch (with permission, Oostergetel \& van Bruggen, 1993). A) amylopectin molecule showing clustering of the $\alpha-(1-4), \alpha-(1-6)$ branch points and the double helical linear glucan chains.

B) crystalline layers containing linear double helical segments in the amylopectin molecules form a continuous network consisting of left-handed helices packed in a tetragonal array. 

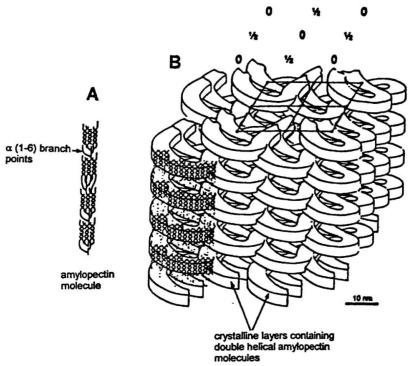
super-helical network of crystalline lamella with left handed helices packed in a tetragonal array. The helical arrangement of crystalline lamellae leaves a void of $8 \mathrm{~nm}$ in diameter.

\subsubsection{Molecular organization of crystalline regions}

Based on the characteristic d-spacings (the distance between planes in the crystal) of wide angle X-ray diffraction patterns, common native starches can be classified into 3 main categories, namely 'A', 'B' and 'C' type [Fig. 2.15]. Cereal starches (rice, normal maize, wheat) show 'A' type X-ray pattern (Fig. 2.15a), whereas tuber starches (potato, tulip, canna, lily) and high amylose maize show 'B' type X-ray pattern (Fig 2.15b). However, some root starches such as tapioca, taro and sweet potato (Takeda et al, 1986) have been found to exhibit ' $A$ ' pattern. ' $C$ ' pattern (Fig. 2.15c), which is a mixture of 'A' and ' $B$ ' type unit cells, has been found in legume (Gernat et al., 1990) and rhizome starches (Zobel, 1988a). In legume starches, 'A' and 'B' type unit cells in starch crystallites have been found in varying proportions. It has been shown that pea starch contains $38.6 \%$ ' $\mathrm{B}$ ' type and $61.4 \%$ ' $\mathrm{A}$ ' type, whereas broad bean starch is composed of $17 \%$ and $83 \%$ of 'A' and 'B' type crystallites respectively (Gernat et al., 1990). Furthermore, Colonna et al. (1981) have reported that pea starch showed $\mathrm{X}$-ray pattern more towards ' $\mathrm{B}$ ' whereas in broad bean starch, the $\mathrm{X}$-ray pattern was more towards ' $\mathrm{A}$ ' type. ' $\mathrm{C}$ ' type has been subdivided into ' $\mathrm{Ca}$ ', ' $\mathrm{Cb}$ ' and ' $\mathrm{Cc}$ ' based on their resemblance to 'A' and ' $\mathrm{B}$ ' types or between the two 
Fig. 2.15 X-ray diffraction patterns of 'A', B' and 'C' type starches with their characteristic d-spacings (adapted from Zobel, 1988b).
A) 'A' type
B) 'B' type
C) 'C' type 


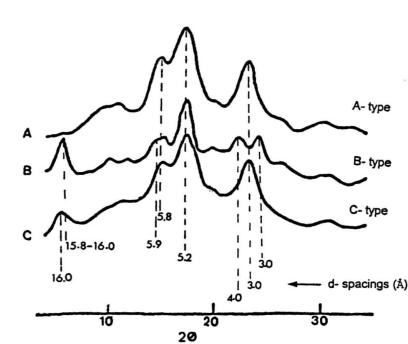


types, respectively (Hizukuri et al, 1960). Hoover \& Sosulski (1985) reported that even legume starches belonging to the same biotype show widely varied $\mathrm{X}$-ray intensities for major peaks, possibly due to the differences in crystallite orientation and/or the amount of ' $\mathrm{A}$ ' and ' $\mathrm{B}$ ' crystallites. The distinguishing features for ' $A$ ', 'B' and ' $C$ ' $X$ - ray patterns are : ' $A$ ' [three peaks at 5.8, 5.2 and 3.8 A (Fig. 2.15a)]; ' $B$ ' [peak at 15.8-16.0 A, a broad medium intensity line at about 5.9A, a strong line at $5.2 \mathrm{~A}$ and a medium intensity doublet at 4.0 and 3.7 A (Fig. 2.15b)]; ' $C$ ' is the same as 'A', except for the addition of a medium to strong peak at $\sim 16.0 \mathrm{~A}$ (Fig. 2.15c); Appearance of this $16.0 \mathrm{~A}$ peak depends on the presence of moisture, and may be missing in dry or partially dry specimens (Zobel, 1988a). The difference in X-ray pattern among starches derives from the way in which the double helices are packed into the unit cells. Therefore, crystallinity in starch is based on the packing arrangement of double helices.

The level of granular crystallinity as measured by $\mathrm{X}$-ray diffraction is in the range of $15-45 \%$ (Table 2.6). Maize and waxy maize starches have the same crystallinity regardless of their amylopectin content [maize $73 \%$ and waxy maize $100 \%]$. Thus, amylose content appears to have little effect on amylopectin crystallinity in the starches that give the 'A' pattern (Zobel, 1988b). 'B' starches show lower crystallinity at increased amylose contents (Table 2.6). Instead of the 'A' pattern (typical for cereal starches), a 'B' pattern is observed for amylomaize (55-75\% amylose) [Table 2.6]. Therefore, it is apparent that in ' $B$ ' type starches low crystallinity is associated with a high amylose content. Zobel 
Table 2.6 Crystallinity of ' $A$ ', 'B'- and 'C' type granular starches Starch Crystallinity (\%) Amylose (\%)

\begin{tabular}{|c|c|c|}
\hline \multicolumn{3}{|c|}{ 'A' starches } \\
\hline Oat & 33 & 23 \\
\hline Rye & 34 & 26 \\
\hline Wheat & 36 & 23 \\
\hline Waxy rice & 37 & - \\
\hline Sorghum & 37 & 25 \\
\hline Rice & 38 & 17 \\
\hline Com & 40 & 27 \\
\hline Waxy maize & 40 & 0 \\
\hline Dasheen & 45 & 16 \\
\hline Nageli amylodextrin & 48 & - \\
\hline \multicolumn{3}{|c|}{ 'B' starches } \\
\hline Amylomaize & $15-22$ & $55-75$ \\
\hline Edible canna & 26 & 28 \\
\hline Potato & 28 & 22 \\
\hline \multicolumn{3}{|c|}{ 'C' starches } \\
\hline Sweet potato & 38 & 20 \\
\hline Horse chestnut & 37 & 25 \\
\hline Tapioca & 38 & 18 \\
\hline
\end{tabular}


(1988a) reported that ' $B$ ' type is more likely to result from the presence of the amylose extender (ae) gene that causes amylopectins to have longer side chains. A waxy genotype (aewx), with no amylose has been shown to give a ' $B$ ' pattern rather than the 'A' pattern of normal waxy starch. However, ' $C$ ' starches do not show a relationship between crystallinity and amylose content (Table 2.6) [Zobel, 1988b].

\subsubsection{Crystal structure of 'A' and 'B' starches}

The detailed structure of 'A' and 'B' unit cells was derived through the collective use of electron diffraction of single crystals, $X$-ray powder patterns, $X$ ray fibre diffraction data (from crystalline amylose) and extensive molecular modeling (French, 1984; Imberty ot al., 1988; Imberty \& Perez, 1996). Kainuma \& French (1972) were the first to suggest that the crystalline orientation of starch was due to both parallel and anti parallel arrangement of double helices. This suggestion was further studied by Wu \& Sarko $(1978 a, b)$ who postulated that both 'A' and 'B' polymorphs are right-handed, parallel-stranded double helices packed in anti parallel manner. The unit cells of 'A' and 'B' starches (Fig. 2.16) were orthogonal and hexagonal, respectively. However, 'A' and 'B' types differ depending on the water content, which is 8 and 36 molecules per unit cell, respectively. Unit cell of 'A' starch has the dimensions of $a=1.19 \mathrm{~nm}, \mathrm{~b}=1.77$ $\mathrm{nm}$ and $\mathrm{c}=1.05 \mathrm{~nm}$. The main objection to this structure was that such anti parallel packing is incompatible with the cluster model of amylopectin. 
Fig. 2.16 Double helix packing arrangement in 'A' and ' $B$ ' type unit cells (adapted from Wu \& Sarko, 1978a; 1987b). 
A Type

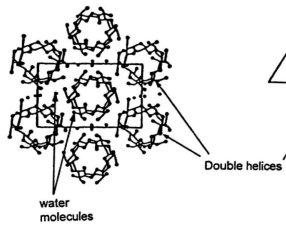

B Type

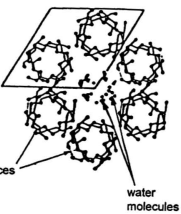


A new model (Fig. 2.17a) of crystalline 'A' starch was proposed by Imberty and co-workers (1987; 1988). The new 'A' unit cell consists of chains which are crystallized in a monoclinic symmetry $(a=2.124 \mathrm{~nm}, b=1.172 \mathrm{~nm}, c=$ $1.069 \mathrm{~nm}$ and $\gamma=123.5^{\circ}$ ) [Fig. 2.17a]. This unit cell has a maltotriose residue as the asymmetric unit, and within the maltotriose residue, all the glucosyl residues are nearly identical. The density calculated for the crystalline region $(d=1.48$ ) was reasonably close to the observed density of the fibre $(d=1.51)$, and indicates that there are 12 glucose residues and 4 water molecules per unit cell. The chain structure is left-handed parallel stranded sixfold double helices packed parallel in the crystalline lattice (Imberty \& Perez, 1996). Each strand repeats in $2.138 \mathrm{~nm}$, but is related to the other strand by a two-fold rotation axis, yielding the apparent fibre repeat distance of $1.069 \mathrm{~nm}$.

There are no intrachain hydrogen bonds, but there is an 0-2.... -6 hydrogen bond between the two strands that contributes about $40 \%$ of the stability of the double helix. The remaining energy of stabilization comes from van der Waals' forces. The double helix is very compact and there is no room for water or any other molecule in its centre (Imberty \& Perez, 1996).

The double helical packing and lattice parameters (Imberty \& Perez, 1988) of the currently accepted 'B' type unit cell (Fig. 2.17b) are in agreement with Wu \& Sarko (1978a) [Fig. 2.16b]. The double helices are left handed, parallel stranded and connected through a network of hydrogen bonds that leaves a channel in the centre of the hexagonal arrangement of six double 
Fig. 2.17 Structure of 'A' and 'B' unit cells.

A) structure of ' $A$ ' unit cell. For each unit cell 4 water molecules $(\cdot)$ are located between the helices (with permission, Imberty et al., 1988).

B) structure of 'B' unit cell. Thirty six water molecules ( $\cdot)$ represent $27 \%$ of hydration (with permission, Imberty \& Perez, 1988).

Hydrogen bonds are shown as broken lines. 

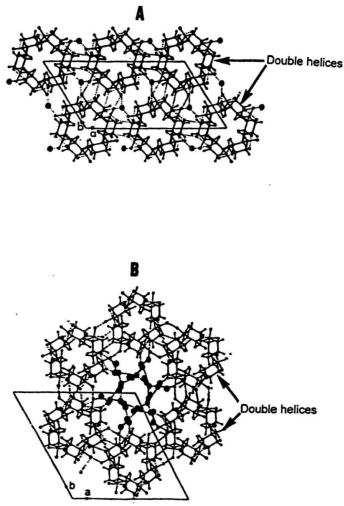
helices. This unit cell $(a=b=1.85 \mathrm{~nm}$ and $c=1.04 \mathrm{~nm}$ ) [Fig. 2.17b] has more open packing of double helices, an asymmetric unit with a maltose residue and 36 water molecules (6 per maltose unit) at $27 \%(w / w)$ hydration. The water molecules are located in fixed positions within this channel. Half of the water molecules are hydrogen bonded to the amylose chains and the other half to other water molecules (Imberty \& Perez, 1988; Imberty \& Perez, 1996). There is no sign of disorder of these water molecules, agreeing with an NMR study which indicates that "freezable" water can be observed only when the hydration is above $33 \%$ (Imberty \& Perez, 1996). The calculated and experimentally determined densities of the unit cell were 1.41 and 1.45 , respectively.

In both ' $\mathrm{A}$ ' and ' $\mathrm{B}$ ' polymorphs, there is a pairing of double helices that corresponds to a $1.1 \mathrm{~nm}$ distance between axes of two double helices. The dense association of this type, which is strengthened by $02 \ldots 06$ and $04 \ldots O 3$ hydrogen bonding, corresponds to the most energetically favoured interactions between two double helices (Perez et al. 1990).

Evidence is also provided by solid state ${ }^{13} \mathrm{C}$ NMR to confirm the asymmetry assignments for 'A' (Fig. 2.18a) and 'B' types (Fig. 2.18b) [Gidley \& Bociek, 1988]. The C-1 signal in 'A' starch spectra (Fig. 2.18a) gives a triplet ( 99.3, 100.4 and $101.5 \mathrm{ppm}$ ) representing 3 residues in maltotriosyl unit, whereas in 'B' starch spectra (Fig. 2.18b), C-1 produces a doublet having shifts at $\sim 100.9$ and $100.0 \mathrm{ppm}$ representing the 2 residues in maltosyl unit. 
Fig. 2.18 ${ }^{13} \mathrm{C}$ CP/MAS NMR spectra of (A) amorphous starch, (B) crystalline 'A' type and (C) crystalline 'B' type starches (adapted from Gidley \& Bociek, 1988). 


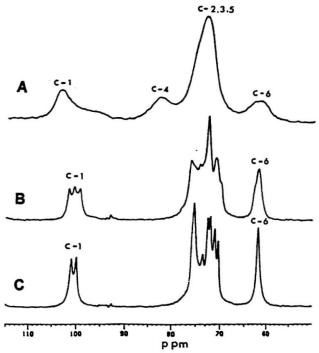


As described before, in the 'A' unit cell (Fig. 2.17a), the centre is filled with a double helix, and the adjacent double helices in crystallites are mainly bonded through hydrogen bonding. In the B structure (Fig. 2.17b) the central open space is filled with water molecules and the double helices are linked by hydrate water bridges. Thus, the ' $B$ ' to 'A' transition (Fig. 2.19) can take place by shifting of helices following removal of water (Zobel, 1988a; Imberty et al., 1991). Under high temperature and low humidity, ' $B$ ' starch may irreversibly be converted to ' $A$ ' starch while remaining in the solid state as fibres or granules. The ' $A$ ' to ' $B$ ' transition is energetically less favourable, therefore, only after 'A' is melted to an amorphous state, crystallization to ' $\mathrm{B}$ ' type is possible (Zobel, 1988a).

The factors affecting the crystalline type of native starches have been examined. Sair (1967) reported that crystallization of starches into 'A', 'B' and 'C' patterns depends mainly on the temperature and water content. Hizukuri (1969) showed that the 'A' type appears in relatively warm $\left(30^{\circ} \mathrm{C}\right)$ conditions and the ' $\mathrm{B}$ ' type in cold $\left(13^{\circ} \mathrm{C}\right)$ conditions. Using linear maltooligosaccharides as model compounds. Gidley $(1987,1992)$ showed that the crystallization of an 'A' type polymorph over ' $\mathrm{B}$ ' type was favoured, under conditions of shorter chain length, higher temperature, higher concentrations, and presence of salts and watersoluble alcohols.

The effect of chain length on the polymorphic form may be rationalized from entropy considerations since with longer chains the entropy changes on 
Fig. 2.19 The transition from 'B' starch to 'A' starch.

Model of the polymorphic transition from ' $\mathrm{B}$ ' to 'A' starch. The water molecules are shown as dots (.) (adapted from Imberty et al., 1991). 


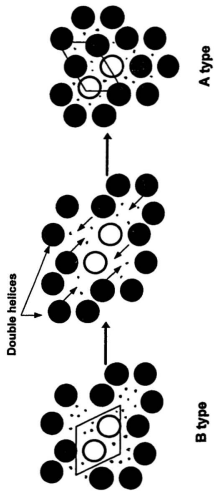


crystallization will become larger and favour the polymorph of highest entropy: i.e. the 'B' type. Hizukuri et al. (1983) and Hizukuri (1986) have also shown that the average chain length of amylopectin is the major determinant of crystalline polymorphism. Based on a study with a series of short chain amyloses of uniform length, Pfannemuller (1987) showed that the degree of crystallinity and the formation of ' $A$ ' and ' $B$ ' type amylose is largely dependent on chain length. The most remarkable observation of this study was an abrupt change from ' $B$ ' to ' $A$ ' pattern in going from DP 13 to DP 12.

\subsubsection{Amorphous region of the granule}

The amorphous region has received scant attention, though it accounts for $\sim 70 \%$ of the granule (Oostergetel \& Van Bruggen, 1993). It has been shown that the amorphous regions are less dense, thus more susceptible to chemical and enzyme attack (Biliaderis, 1982). Absorption of cold water by amorphous regions allows the limited reversible swelling of starch granules (French, 1984). Diffusion of small water soluble molecules $(<1000$ dalton) into the granule occurs through the amorphous region. Gidley \& Bociek (1988) reported that ${ }^{13} \mathrm{C}$ CP/NMR spectra of amorphous starch (Fig. 2.18c) showed substantial differences compared to crystalline starch (Fig. 2.18a,b). Conformational differences between amorphous and crystalline regions were shown by chemical displacements, especially at the $\mathrm{C}-1$ and $\mathrm{C}-4$ sites. In $\mathrm{C}-1$ region, peak intensity in amorphous region (Fig. 2.18c), was shifted to a low field compared to 
crystalline region (Fig. 2.18a,b), and the amorphous spectrum (Fig. 2.18c) showed a peak at $81-83 \mathrm{ppm}$, which was absent in crystalline material. The signals at 81-83 ppm (C-1), 94-98 ppm and 102-105 ppm (C-4) were assigned to amorphous sites (Fig. 2.18c).

The arrangement of amylose and amylopectin within the amorphous regions has been the subject of much controversy. Blanshard (1987) and Zobel (1988a) reported that in com and wheat starches amylose is separated from amylopectin, whereas in potato starch, part of the amylose seems to be cocrystallized with amylopectin. Light cross linking and characterization of products by molecular sieve chromatography (Jane et al., 1992; Kasemsuwan \& Jane, 1994) showed that in potato and com starches, amylose was cross linked with amylopectin, but no cross linking occurred between amylose molecules. This observation suggested that amylose molecules in the amorphous regions are interspersed among amylopectin, but do not exist in the form of bundles. Thus, it is likely that some amylose may form double helices with amylopectin and become less prone to complex formation with iodine or leaching in the presence of warm water. Vasanthan \& Hoover (1992a) suggested that the extent to which the starch components are associated with each other within the native granule may also depend on their respective average $\mathrm{CL}$. Long amylose chains may facilitate easier association with the short chain (DP 20-25) amylopectin molecule. The above authors postulated that in potato starch, the degree of 
association between starch components may be higher than in other starches due to its long amylose $\mathrm{CL}$ (Table 2.4). 


\subsection{Starch properties}

\subsubsection{Gelatinization}

Native starches are generally insoluble in cold water, but granules undergo slight reversible swelling (10-20\%) due to absorption and diffusion of water into the amorphous regions (Biliaderis, 1991). On heating, starch granules in an aqueous starch suspension undergo an order-disorder phase transformation termed gelatinization (Fig. 2.20) [Donovan, 1979]. This phase transition is associated with a significant uptake of water which results in irreversible granular swelling, loss of birefringence, loss of crystalline order, leaching of amylose into the solution and increase in viscosity (Donovan, 1979; Hoover \& Hadziyev, 1981). An individual granule gelatinizes over a narrow temperature range of $0.5-1.5^{\circ} \mathrm{C}$, whereas a population of starch granules gelatinizes over an approximate range of $10^{\circ} \mathrm{C}$ (Gough \& Pybus, 1971).

In studying the gelatinization phenomenon, many researchers (Biliaderis et al., 1986b; Russell, 1987) have applied the Flory-Huggins (Flory, 1953) equation to relate melting of starch crystallites to the amount of water, assuming that starch-water system is homogenous and gelatinization occurs under equilibrium conditions. This theory describes the depression of the true melting point of a polymer $\left(T_{m}{ }^{9}\right)$ to the melting point of the polymer diluent mixture $\left(T_{m}\right)$ as a function of the volume fraction of the diluent.

$1 / T_{m}-1 / T_{m}{ }^{\circ}=\left(R / \Delta H_{u}\right)\left(V_{u} / V_{1}\right)\left(V_{1}-\chi_{1} V_{1}{ }^{2}\right) \quad$ Flory-Huggins equation 
Fig. 2.20 Schematic presentation of the changes which occur in the starch granule during gelatinization (adapted from Aguilera \& Stanley, 1990; Biliaderis, 1991). 


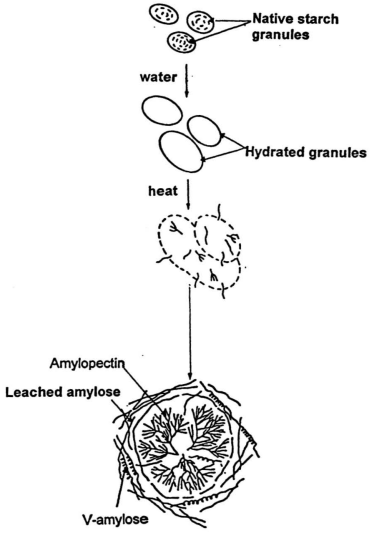

Gelatinized granules 
$\Delta \mathrm{H}_{\mathrm{u}} \quad$ = change in enthalpy of fusion per repeating unit (glucose)

$V_{u} / V_{1}=$ ratio of the molar volume of the repeating unit (glucose) in the chain to that of the diluent (water)

R = gas constant

$T_{m} \quad=$ melting point of the diluent-polymer mixture

$\mathrm{T}_{\mathrm{m}}{ }^{\circ}=$ true melting point of the undiluted polymer

$v_{1}=$ volume fraction of the diluent [volume of water/(volume of water + volume of starch)]

$\chi_{1}=$ polymer solvent interaction parameter

Since the Flory-Huggins theory is applicable only to equilibrium crystals, its application to phase transition of starch water system has only a limited success. However, Lelievre (1976) reported that equilibrium conditions in heated aqueous starch systems can be approached using very slow heating rates $\left(-1^{\circ} \mathrm{C}\right.$ /3h). Evans \& Haisman (1982) reported that the starch-water system is not homogenous, due to the fact that it consists of individual granules suspended in a variable amount of liquid phase. Once these starch granules which are in osmotic equilibrium with external phase are fully swollen, further addition of the liquid phase will not affect granule composition. Therefore, volume fractions for the Flory-Huggins equation should be based on granule composition rather than the composition of the entire system. Whittam et al. (1991) suggested that even with highly crystalline preparations of A- or B- type starch crystals (DP 15), the 
estimates of $\mathrm{T}_{m}{ }^{\circ}$ obtained for starch crystallites using Flory-Huggins approach may be somewhat low.

The amorphous regions and the crystallites are not independent of each other, but are interconnected in the starch granule. The amorphous regions are in a glassy state and their transition temperature (the glass transition temperature: $T_{g}$ ) is higher than the melting temperature of the crystallites in native starch granule. However, the amorphous regions must first undergo a transition from a glassy state to a rubber-like state before the crystallites can melt, i.e. the crystallites are kinetically stabilized in the native starch granule by the amorphous region. The amorphous regions are always hydrated first (van den Berg, 1986) and the water acts as a plasticizer and depresses the $T_{g}$ below the melting temperature of crystallites. These are now less kinetically constrained, and melt at temperature slightly higher than the $T_{g}$. The glass transition always precedes gelatinization and determines the start of the gelatinization process (Slade \& Levin, 1987; Slade \& Levin, 1988). Since water has to penetrate the starch granule from the outside, the amorphous regions are only partially hydrated at the beginning of gelatinization and the 'effective $T_{g}$ ' (which determines the start of gelatinization) of each type of starch is therefore, more or less the same (Biliaderis, 1990).

Several analytical techniques including viscometry, X-ray diffraction, DSC, light and electron microscopy and NMR have been employed to understand the mechanism of starch gelatinization. Because gelatinization is an endothermic 
process, DSC has been widely used to study phase transitions of aqueous starch suspensions. DSC can provide the characteristic temperatures and enthalpies of the various transitions as well as allowing measurements over a wide range of starch concentrations.

At relatively high moisture levels (volume fraction of water $>0.7$ ). DSC thermograms of starches show a single endotherm at about $60^{\circ} \mathrm{C}$ (Fig. $2.21 \mathrm{a}$ ). The position of the peak depends on the starch variety being investigated (Biliaderis et al., 1986b). As the water content is reduced (at intermediate moisture levels, volume fraction $\sim 0.6$ ), two endothermic transitions become evident (Fig. 2.21b) [Donovan, 1979; Hoseney et al., 1986; Blanshard, 1987]. Donovan (1979) designated the initial peak and the second peak as $G$ and $M$, respectively. The $G$ endotherm occurs at the same temperature as before. The temperature of the second endotherm $(M)$ increases as the water content decreases. If the volume fraction $(v)$ of water is decreased still further $(v<0.45)$. the lower temperature endotherm (G) disappears (Fig. 2.21c), while the temperature of the second peak continues to rise (Fig. 2.20d) [Donovan, 1979]. In addition to the above endotherms, transitions due to lipid- amylose complexes are found at about $120^{\circ} \mathrm{C}$ (Biliaderis et al., 1986ac; Biliaders \& Seneviratne, 1990). The exact temperature depends on the moisture content.

Donovan (1979) suggested that the single peak ( $G$ endotherm) at excess moisture content (Fig. 2.21a) results from the 'stripping' or unfolding of polymer chains from the surfaces of crystallites due to stress developed by hydration and 
Fig. 2.21 DSC melting profiles of potato starch at various volume fractions of water (adapted from Donovan, 1979).
A) 0.81
B) 0.55
C) 0.38
D) 0.28 


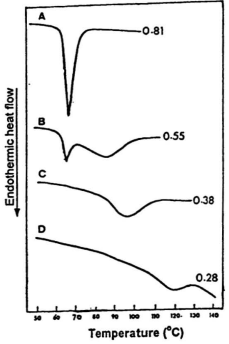


swelling of amorphous regions, while the second peak represents melting at low diluent volume fractions. Since Donovan (1979) considered different mechanisms to be responsible for each section of the biphasic endotherm, the peaks were designated as $\mathrm{G}$ and $\mathrm{M}$, respectively. However, Zobel et al. (1988) have postulated that the $\mathrm{X}$ - ray data do not indicate that different molecular processes are responsible for each peak.

Evans \& Haisman (1982) explained the appearance of the biphasic endotherm based on water migration. Water migrates within the sample from one location to another, and the peaks represent the order-disorder transitions occurring at different diluent levels. Starch granules can take up only a limited amount of water and the additional water added to the system forms a separate phase. The crystallites in granules are of a range of stabilities and the least stable crystallites melt first when heat is applied. When crystallites melt, the deformed polysaccharide chains absorb more water from the separate phase because it is slightly more flexible. Then, the additional water will lower the stability of the remaining crystallites, thereby the crystallites will melt at a higher temperature. This theory was supported by Liu ot al. (1991) who reported that water gradient within the sample is responsible for the biphasic transition and the crystallinity is lost when gelatinization proceeds. The theory based on the migration of water is also in agreement with the study conducted by Zobel et al. (1988), which shows the occurrence of $X$-ray intensity changes corresponding to the development of first and the second peaks, based on the 
melting of crystallites with different stabilities. Russell (1987) ascribed the biphasic endotherm to disruption of double helices associated with short-range order involving amylose and amylopectin followed by melting of crystallites. Biliaderis (1990) suggested that a process of partial melting followed by recrystallization and final melting is the reason for biphasic thermal profiles. Recently. Svensson \& Eliasson (1995) attributed the biphasic endotherm to a slow plasticization of the amorphous granular regions under restricted water conditions which forces the melting of crystallites to higher temperatures.

Several workers have postulated that the enthalpy of gelatinization isrelated to the disruption or melting of organized structures (Donovan, 1979; Zobel et al., 1988; Whittam et al., 1990; Gidley, 1992). However, Cooke \& Gidley (1992) using ${ }^{13} \mathrm{C}$ MAS NMR, showed that double helix melting rather than loss of crystallinity, could be primarily responsible for gelatinization enthalpy.

Some researchers have attempted to explain the development of biphasic endotherm based on the glass transition which defines the temperature region in which the chain motion of a polymer commences. This theory suggests that the initial (G) peak is a result of chain mobilization in the amorphous regions of the starch granule. With the increase in mobility, the polymer chains are transferred from a glassy to a rubbery aqueous gel (Biliaderis et al., 1986b; Yost \& Hoseney. 1986). Several investigators (Biliaderis, 1991; Zeleznak \& Hoseney, 1987) suggested that glass transition is located at the leading edge of the first peak (Fig. 2.22) and it is associated with a change in heat capacity. The completion of 
Fig. 2.22 Thermal profiles of rice starch (at volume fractions of water 0.50 and 0.70 ) showing both glass transition $(\mathrm{Tg}=$ glass transition temperature) and melting transition (with permission, Biliaderis, 1986b). 


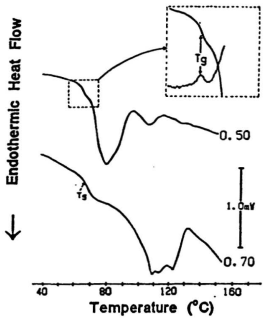


glass transition allows the crystalline domains to undergo a non equilibrium melting process giving the second endothermic peak (Slade \& Levine, 1988). However, some studies have provided data contrary to this theory, showing that heat capacity changes occur throughout the temperature range of both endotherms (Lelievre, 1992). Liu et al. (1991) demonstrated, using X-ray diffraction studies, that the heat capacity change is not related to glass transition due to two reasons; firstly, the X-ray data do not show that a significant endothermic transition occurs without a corresponding change in crystallinity. secondly, the $\mathrm{X}$-ray data suggest that the volume expansion measured by other investigators (Biliaderis of al., 1986b) using thermomechanical analysis are attributable to the increase in the quantity of amorphous polymer with the temperature rather than to a glass transition followed by melting.

A study (Jang \& Pyun, 1996) of wheat starch in the presence of limited water (40,50\% moisture) showed the appearance of four endotherms (Fig. 2.23a) : $G$ (water mediated melting of starch crystallites), $M_{1}$ (melting of the remaining crystallites or amylopectin crystallites), $\mathrm{M}_{2}$ (melting of amylose-lipid complex) and $\mathrm{M}_{3}$ (melting of amylose). These results were in agreement with the study conducted by Donovan \& Mapes (1980). For moisture contents less than $30 \%$, (Fig. 2.23b) the $\mathrm{G}$ and $\mathrm{M}_{1}$ endotherms shifted to a higher temperature and at moisture contents below $20 \%, G$ and $M_{1}$ coalesced into a single band $\left(G+M_{1}\right)$ and $M_{2}$ and $M_{3}$ coalesced into a single band $\left[M_{2}+M_{3}\right]$ (Jang \& Pyun, 1996). Biliaderis et al. (1986a) and Biliaderis \& Galloway (1989) attributed the $\mathrm{M}_{2}$ and 
Fig. 2.23 DSC thermograms of wheat starch at various water contents (adapted from Jang \& Pyun, 1996).
A) DSC profiles of wheat starch at water contents varying from $30-90 \%$.
B) DSC profiles of wheat starch at water contents varying from $2.8-25 \%$.
$\mathrm{G} \& \mathrm{M}_{1}$ : melting of amylopectin crystallites at intermediate moisture content.
$M_{2}$ : melting of amyiose - lipid complex
$\mathrm{M}_{3}$ : melting amylose 
Ma to melting of amylose - lipid complexes (differ in the degree of helical chain organization), since waxy or defatted starches do not exhibit the $M_{2}$ or $M_{3}$ transitions (Donovan et al., 1983).

\subsubsection{Factors influencing Gelatinization}

It has been shown that melting parameters (transition temperatures and enthaipy) are influenced by factors such as botanical source (granule structure). moisture content, heating rate, starch modification, lipids, sugars, mechanical damage, etc.

\subsubsection{Botanical source}

Crystal size, perfection of ordered chains and the amorphous domains contribute to the thermal stability of granular starch. Granular 'A' starches usually exhibit lower melting temperatures than 'B' starches (Table 2.7) [Whittam et al,, 1990]. However, highly crystalline 'A' and ' $B$ ' spherulites obtained from potato starch lintners (DP 15) showed similar melting enthalpy (35 J/g) and a difference of $15-20^{\circ} \mathrm{C}$ in a fixed water content (Whittam et al., 1990). In contrast to native starch crystals, 'A' crystals of lintners showed a higher melting temperature than 'B' type crystals (Whittam et al., 1990). Variations in melting parameters (Table 2.7) have been observed among different genotypes of rice, barley, corn and legume starches (Hoover \& Sosulski, 1985; Morrison et al., 1993a; Yuan et al.,1993,). Yuan et al. (1993) studied the gelatinization 
$M_{3}$ to melting of amylose - lipid complexes (differ in the degree of helical chain organization), since waxy or defatted starches do not exhibit the $M_{2}$ or $M_{3}$ transitions (Donovan et al., 1983).

\subsubsection{Factors influencing Gelatinization}

It has been shown that melting parameters (transition temperatures and enthalpy) are influenced by factors such as botanical source (granule structure), moisture content, heating rate, starch modification, lipids, sugars, mechanical damage, etc.

\subsubsection{Botanical source}

Crystal size, perfection of ordered chains and the amorphous domains contribute to the thermal stability of granular starch. Granular 'A' starches usually exhibit lower melting temperatures than ' $B$ ' starches (Table 2.7) [Whittam et al., 1990]. However, highly crystalline 'A' and ' $B$ ' spherulites obtained from potato starch lintners (DP 15) showed similar melting enthaipy $(35 \mathrm{~J} / \mathrm{g})$ and a difference of $15-20^{\circ} \mathrm{C}$ in a fixed water content (Whittam et al., 1990). In contrast to native starch crystals. 'A' crystals of lintners showed a higher melting temperature than 'B' type crystals (Whittam et al., 1990). Variations in melting parameters (Table 2.7) have been observed among different genotypes of rice, barley, corn and legume starches (Hoover \& Sosulski, 1985; Morrison et al., 1993a; Yuan et al.,1993.). Yuan et al. (1993) studied the gelatinization 
Table 2.7 Gelatinization parameters (at excess water) of some cereal tuber and legume starches

\begin{tabular}{lcccl}
\hline Starch & $\begin{array}{c}\text { X-ray } \\
\text { diffraction } \\
\text { pattern }\end{array}$ & $\begin{array}{c}\text { Gelatinization } \\
\text { peak } \\
\text { temperature }\end{array}$ & $\begin{array}{c}\text { Enthalpy of } \\
\text { gelatinization } \\
(\mathrm{J} / \mathrm{g})\end{array}$ & \\
\hline Oat (Svea) & $\mathrm{A}$ & 58 & 10.6 & Gudmundsson \& Eliasson, 1989 \\
Oat (Chichauaua) & A & 62 & 10.1 & Gudmundsson \& Eliasson, 1989 \\
Rye & A & 58 & 11.9 & Gudmundsson \& Eliasson, 1991 \\
Wheat & A & 58 & 9.7 & Gidley, 1992 \\
Maize & A & 58 & 14.3 & Cooke \& Gidley, 1992 \\
Waxy maize & A & 72 & 16.0 & Cooke \& Gidley, 1992 \\
Waxy rice (RD6) & A & 64 & 13.4 & Tester \& Morrison, 1990b \\
Waxy rice (IR29) & A & 66 & 14.2 & Tester \& Morrison, 1990b \\
Amylomaize & B & 82 & 10.0 & Gudmundsson \& Eliasson, 1989 \\
Edible canna & B & 70 & - & Zobel 1988b \\
Potato & B & 58 & 16.2 & Cooke \& Gidley, 1992 \\
Kidney beans & C & 70 & 15.1 & Hoover \& Sosulski, 1985 \\
Navy beans & C & 68 & 13.4 & Hoover \& Sosulski, 1985 \\
Black beans & C & 66 & 12.6 & Hoover \& Sosulski, 1985 \\
Smooth pea & C & 64 & 14.7 & Biliaderis ot al., 1980 \\
Smooth pea & C & 61 & 13.4 & Colonna et al., 1987 \\
Lentil & C & 57 & 14.2 & Biliaderis $\theta t$ al., 1980 \\
Sweet potato & C & 70 & - & Zobel 1988b \\
Tapioca & C & 66 & - & Zobel 1988b \\
\hline
\end{tabular}


parameters of three genotypes (wx, aewx, duwx) of waxy corn starch and reported that the aewx $\left(T_{p}=79^{\circ} \mathrm{C}\right)$ had the highest melting temperature and $\Delta \mathrm{H}$ (4.1 Cal/g) among the three genotypes (for $w x, T_{p}=66.9^{\circ} \mathrm{C}, \Delta H=3.2 \mathrm{Cal} / \mathrm{g}$; for duwx, $T_{p}=70^{\circ} \mathrm{C}, \Delta H=3.2 \mathrm{Cal} / \mathrm{g}$ ). The higher melting temperature of aewx was attributed to aewx having amylopectin with longer chains, which could account for the ' $B$ ' type crystallites.

\subsubsection{Heating rate}

Calorimetric studies of Shiotsubo \& Takahashi (1984) has shown that the peak temperature of gelatinization endotherm increases with increasing heating rate. The peak temperature remained constant for heating rates below $0.5^{\circ} \mathrm{C}$ / min. Biliaderis et al. (1986b) reported that the biphasic endotherm observed at 3$20^{\circ} \mathrm{C} /$ min merged into a single endotherm at increased heating rates. Since reorganization process is time-limited, the melting probably occurs as a single stage process at high heating rates (Biliaderis ot al., 1986b).

\subsubsection{Lipids}

The effect of lipids on gelatinization has been discussed in section 2.4.2.2.4. 


\subsubsection{Sugars}

In general, sugars hinder granule swelling and increase gelatinization temperatures (Evans \& Haisman, 1982; Eliasson, 1992: Bello-Perez \& ParedesLopez, 1995). Sucrose hindered gelatinization and increased melting temperature from 57 to $92^{\circ} \mathrm{C}$ at concentrations of $55-60 \%$ (Kim \& Walker ,1992). Scanning electron micrographs showed that the granules isolated from baked products with high sucrose contents were less deformed compared to those obtained from baked products with low sucrose contents (Hoseney et al., 1978). However, unaffected and increased gelatinization enthalpies in the presence of sugars have also been reported by Evans \& Haisman (1982) and Eliasson (1992), respectively. Several theories have been presented for the influence of sugars on retarding gelatinization, which include the competition between starch and sugar for water (D'Appolonia, 1972) and sugar-starch interactions (Lelievre, 1976).

\subsubsection{Starch modification}

Physical or chemical modification of starch by changing the molecular order while maintaining the granular form is applied in production (atomization and heating in aqueous monohydric alcohol solutions) of granular cold-water soluble starch which gelatinize in warm water (Jane et al., 1986). Heat-moisture treatment, annealing, defatting, acetylation, hydroxypropylation, cross linking etc. have been shown to alter gelatinization parameters of starches (Wootton \& 
Bamunuarachchi, 1979; Hoover \& Sosulski, 1985,1986; Hoover \& Vasanthan, 1994a; Hoover \& Manuel, 1996a,b). Effect of physical and chemical modification on gelatinization parameters will be discussed in section 2.6 in detail.

\subsubsection{Retrogradation}

Starch granules heated in excess water undergo an order-disorder phase transition called gelatinization over a temperature range characteristic of the starch source. This phase transition is a non equilibrium process associated with the diffusion of water into the granule, hydration and swelling of starch granules, uptake of heat, loss of crystallinity and amylose leaching (Donovan, 1979; Hoover \& Hadizeyv, 1981; Biliaderis, 1990). On cooling, amylose and amylopectin chains in the gelatinized paste associate, forming a more ordered structure (Fig. 2.24-1). These molecular interactions are termed collectively "retrogradation" and have important textural and dietary implications. In order to understand the process of aging, the starch/water system has been extensively investigated using physical methods such as turbidity (Miles et al., 1985a: Ring et al., 1987; Jacobson et al., 1997), DSC (Mclver et al., 1968; Longton \& LeGrys, 1981: Russell, 1987), theology (Miles et al., 1985a,b; l'Anson, ot al., 1988). X-ray diffraction (l'Anson, et al., 1988), microscopy (Jacobson et al., 1997). FTIR (Wilson et al., 1991; Van Soest et al., 1995) and NMR spectroscopy (Wu \& Eads, 1993) to measure different properties occuring during retrogradation. For example turbidity measures distribution of refractive index (hence density), DSC 
Fig. 2.24 Mechanisms of starch retrogradation.

(I) Schematic illustration of changes during storage of gelatinized starch paste (adapted from Aguilera \& Stanley ,1990; Biliaderis, 1991).

(II) double helix formation and association : (a) helix formation and chain elongation; (b) lateral association of helical regions (with permission, Morris, 1990), 


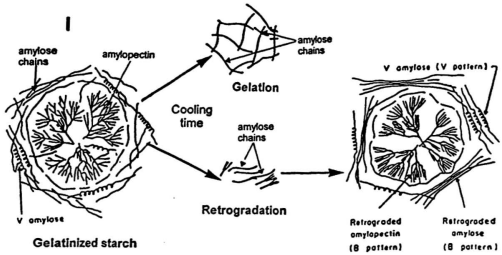

II

(a)

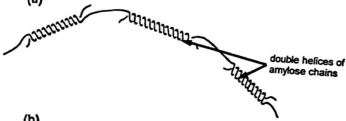

(b)

(B) pottarn!

(日) potiern)

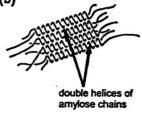


measures melting transitions, X-ray diffraction monitors long-range order in crystalline starch domains, microscopy is used to understand the spatial distribution of refractive mass and NMR monitors chain segmental motions, conformation dependent chemical shifts (resonance frequencies) and degree of crystallinity.

The molecular structures and transformations that occur during retrogradation of starch and its components have been subjected to several investigations. Despite the numerous investigations that have been carried out to study retrogradation, the exact mechanism of retrogradation, particularly at the molecular level still remains unclear. The rate and extent of retrogradation is influenced by many factors such as botanical source (Orford et al., 1987: Jacobson et al., 1997), chain length of amylose and amylopectin (Gidley \& Bulpin, 1989; Clark et al., 1989), water content in gel (Longton \& LeGrys, 1981; Zeleznak \& Hoseney, 1986), cooking and cooling conditions (Kim et al., 1993), storage temperature (Jankowski \& Rha, 1986; Jang \& Pyun, 1997) and the presence of solutes such as sugars, lipids and salts (Russell \& Oliver, 1989; Katsuta et al., 1992a,b; Huang \& White, 1993; Conde-Petit \& Escher, 1994). This review summarizes the contribution of amylose and amylopectin to starch retrogradation. 


\subsubsection{Amylose Gelation}

Amylose gelation is characterized by the formation of a permanent elastic network and the development of opacity (Miles et al., 1985a). Amylose gelation occurs above its critical overlap or entanglement concentration $\left[C^{*} \sim 1.5 \%\right.$ ]: which defines the minimum concentration for gelation (Miles et al., 1985a). Below $\mathrm{C}^{*}$, amylose precipitates forming insoluble crystals which melt at 150 $160^{\circ} \mathrm{C}$ (Miles et al., 1985a; Stute \& Konieczny-Janda, 1983). However, amylose gelation was found to proceed even at a concentration below $\mathrm{C}^{*},(1 \%)$ regardless of the molecular weight (Dublier \& Choplin, 1989). Amylose gelation is favoured by long CL (DP >1100), high concentrations and fast cooling rates, whereas precipitation of amylose is favoured by shorter $\mathrm{CL}$ (DP $<110$ ), low concentration and slow cooling rates (Gidley \& Bulpin, 1989). Both gelation and precipitation occur for CL of 250-660 residues (Gidley \& Bulpin, 1989). The above authors postulated that gelation involves extensive cross linking (via hydrogen bonding and/or hydrophobic interactions) between long amylose chains, which leads to the formation of a macromolecular network. Thus, if the total chain length is greater than the length of the chain segments which participate in the above interactions, then cross linking would probably involve more than two regions within a single amylose chain. For intermediate chains (DP 250-660), both chain alignment and cross linking can occur. However, if the length of interacting chains are approximately of the same length as the total chain length, then chain alignment would occur in preference to cross linking. 
Cross linking is favoured by higher concentrations (greater interchain contact) and rapid cooling (increases nucleation). Whereas slow cooling may lead to annealing of the structure favouring chain alignment (Gidley \& Bulpin, 1989). Chain alignment followed by lateral association predominates in shorter chains (DP < 110). This leads to precipitation of amylose (Gidley \& Bulpin, 1989).

Amylose gels are also characterized by the development of turbidity, which is caused by phase separation which forms polymer rich and polymer deficient regions (Miles et al., 1985a). This process depends on polymer concentration, molecular size and cooling temperature. Gidley (1989) suggested that amylose gelation is due to interchain associations in the form of double helices, followed by aggregation of helices which act as junction zones. Morris (1990) postulated that in amylose gelation, double helix formation can occur between the ends of molecules favoring chain elongation (Fig. 2.24-Ha). Once the helices are formed, lateral association may occur through crystallization (Fig. 2.24-llb). Based on the results of stored amylose ( $21^{\circ} \mathrm{C}$ for 5 weeks), Muller et al. (1995) postulated that aged amylose gels may contain double helices, small aggregates of double helices (junction zones), crystallites and their aggregates.

Amylose gels are poorly crystalline structures, largely composed of amorphous regions (Miles et al., 1985a). The development of crystallinity in polymer rich phase was shown to be a slow process (Miles et al., 1985a) in which the rate was concentration independent. The overall level of crystallinity 
was shown to be concentration dependent (Miles et al., 1985a). Miles et al. (1985a) reported that the development of crystallinity is accompanied by stiffening of amylose gels. X-ray diffraction (Leloup et al., 1991: Caims et al., 1995) and ${ }^{13} \mathrm{C}$ NMR (Colquhoun et al., 1995) have shown that retrograded amylose is composed of ' $\mathrm{B}$ ' type crystals. In the crystalline domains, amylose double helical structures aggregate into compact arrays (Imberty \& Perez, 1988). Helical packing becomes more perfect for shorter chain lengths (Gidley ,1989). The crystalline regions of amylose gel are resistant, whereas the amorphous regions are easily degraded by acidic and amylolytic hydrolyse (Leloup et al.. 1991). The amount of the crystalline region in retrograded amylose can be as high as $65-83 \%$ of the gel (Leloup et al., 1991: Caims et al., 1995).

Clark et al. (1989) reported that although all gelling amylose exhibit turbidity development, variations exist with respect to the time scale of modulus (which measures gel stiffness) and absorbance development as a function of chain length. For short chains (DP $250 \& 300$ ) the increase in turbidity precedes modulus development. suggesting that some non cross linking aggregation (precipitation) occurs. However, for longer chains (DP >1100) modulus increases before significant turbidity is apparent. This suggests that the processes which lead to gelation and turbidity in aqueous amylose systems are not directly related, although turbidity development was ascribed to helix - helix aggregation (Gidley, 1989). 
Leloup et al. (1992) investigated the structural characteristics of amylose gels $(2-8 \% w / v)$ of smooth pea starch by electron microscopy, mild acid hydrolysis, DSC and size exclusion chromatography. The results showed a macroporous structure in gel (mesh size 100-1000 nm) with filaments $20 \pm 10$ $\mathrm{nm}$ wide (Fig. 2.25). The filaments were composed of association of amylose chains with $D P_{n} 26-31$ and $D P_{w}$ 56-73. The double helices in the filaments linked to each other by loops of amorphous amylose segments, which are dangling in the gel pores. The aggregation of these filaments generates a three dimensional network consists of network strands, amorphous zone and intermediary zone (Fig. 2.25).

\subsubsection{Amylopectin Gelation}

Compared to amylose, amylopectin retrogradation is a very slow process. Amylopectin gels are turbid and elastic, and the gels form on cooling of substantially higher polysaccharide concentrations (> $10 \%$ w/w) to $2{ }^{\circ} \mathrm{C}$ (Ring et al., 1987). The development of gel stiffiness was attributed to associations involving crystallization process, which gives an X-ray pattern characteristic of the 'B' type (Ring et al., 1987; Eerlingen et al., 1994). Crystallization and the increase in stiffness in amylopectin gels can be reversed by heating to $100^{\circ} \mathrm{C}$ (Miles et al., 1985b; Durani \& Donald, 1995).

Gelling behaviour of amylopectin is influenced by the fine structure (botanical source) of amylopectin (Kalichevsky et al., 1990). Amylopectins from 
Fig. 2.25 Continuous model for amylose gel (with permission, Leloup et al., 1992) 


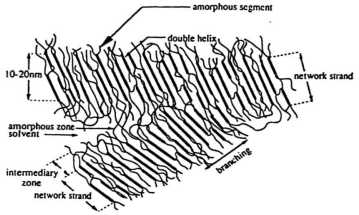


potato, pea and canna were found to exhibit higher rates of retrogradation than those from wheat, barely and maize, presumably due to the shorter CL in cereal starches (Table 2.4) [Kalichevshy ot al., 1990]. Ward ot al. (1994) demonstrated that corn amylopectin retrogrades faster than wheat amylopectin. This was attributed to the greater proportions of chains with DP 15-20 in corn amylopectin. The above authors postulated that the extent of retrogradation is increased by high molar proportion of unit chains with DP 14-24, and decreased by a low molar proportion of short chains with DP 6-9. This was in agreement with the finding of Ring (1987) and Wursh \& Gumy (1994) who reported that retrogradation is hindered in the presence of short amylopectin chains with DP 11 or less. A study with starches from different rice cultivars showed that amylopectin gels from Japonica and low amylose Indica had higher retrogradation rates compared to amylopectin gels of waxy rice (Lu et al., 1997). This was attributed to greater proportion (63-66\%) of short chains (DP 10-15) in japonica and indica. Furthermore, waxy rice amylopectin also contained a greater proportion of very short chains (DP 6-9), which were shown to hinder retrogradation (Lu et al., 1997). In a study with waxy maize amylopectin, Ring et al. (1987) observed a broad retrogradation endotherm for $20 \%$ amylopectin gel stored for 4 weeks at $1^{\circ} \mathrm{C}$, and a sharp peak for gelatinization of waxy maize starch dispersion (Fig. 2.26). The mid point transition temperatures for gel and starch were 54 and $75^{\circ} \mathrm{C}$, respectively. Both retrogradation $(1.5 \mathrm{~mJ} / \mathrm{mg})$ and gelatinization (1.54 $\mathrm{mJ} / \mathrm{mg})$ transitions gave similar enthalpy values. Durani \& 
Fig. 2.26 DSC thermograms showing gelatinization of waxy starch and melting of $20 \%$ waxy-maize amylopectin gel stored for 4 weeks at $1^{\circ} \mathrm{C}$ (adapted from Ring et al., 1987) 


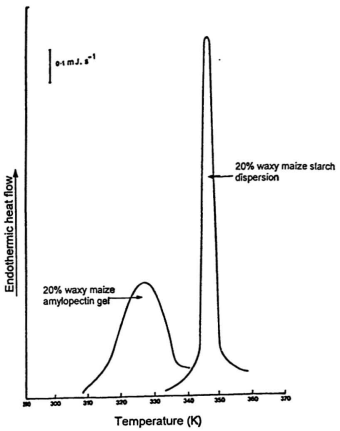


Donald (1995) reported that amylopectin gels (formed with amylopectins of different molecular weights) stored at $4^{\circ} \mathrm{C}$ melt over a broad range of temperatures. The melting enthalpies of gels made from higher molecular weight amylopectin were higher than those of their lower molecular weight counterparts.

FTIR spectroscopic studies revealed chain ordering at the very early stages of amylopectin gelation (complete within $0.2 \mathrm{~h}$ for a $20 \%$ waxy maize gel) which was not detectable by other methods (Goodfellow \& Wilson, 1990). The above authors attributed this chain ordering to coil to double helix transitions in short DP chains of amylopectin. This transition was followed by a slow aggregation of the helices to produce crystallites. Biliaderis \& Zawistowski (1990) studied the storage modulus and melting enthalpy of aging waxy maize (amylopectin) gels $(40 \% \mathrm{w} / \mathrm{w})$. The results showed that the storage modulus reached a limiting value after $36 \mathrm{~h}$ at $6^{\circ} \mathrm{C}$, but $\Delta \mathrm{H}$ continued to increase over a long time scale. Based on the results, the above authors postulated that once the gel network is developed, subsequent ordering and crystallization of amylopectin chains do not contribute significantly to gel rigidity. Similar time dependence was reported for a $20 \%$ amylopectin solution stored at $1^{\circ} \mathrm{C}$ by Ring et al. (1987) who observed that the increase in turbidity values stopped after 4-5 days, whereas DSC and X-ray diffraction data continued changing over a long period of time ( $30-40$ days). The above authors suggested that increase in turbidity reflected the aggregation of amylopectin chains prior to gelation and the long term gel network development (as measured by DSC and X-ray diffraction) 
showed the ordering and crystallization of short (DP 10-20) amylopectin chains. Studies on aging waxy maize $\left(20,40 \%\right.$ w/w) starch gels using ${ }^{1} \mathrm{H}$ NMR (Wu \& Eads, 1993) showed that the polymer chains in starch gels exhibit three types of mobile fractions : 1 ) a highly mobile chain fraction, 2) highly rigid (the motion of the segments is highly restricted) regions corresponding to crystalline domains. and 3) a fraction with intermediate mobility (motions are intermediate between those in crystalline and dissolved states). The component with intermediate mobility has been shown to correspond to unassociated or partially associated chains in polymer rich regions. During the aging process the immobile fractions of starch increase and the mobile fractions decrease (Wu \& Eads, 1993). Cameron et al. (1994) demonstrated the establishment of network structures, involving only short range intermolecular associations possibly via double helix formation.

\subsubsection{Retrogradation of starch}

Starch gels are formed when gelatinized starch dispersions $(>6.0 \% \mathrm{w} / \mathrm{w})$ are cooled to room temperature (Ring, 1985). On cooling the paste, the exuded amylose forms an interpenetrating network in which the gelatinized granules rich in amylopectin are embedded. Such a matrix is regarded as a composite material and its mechanical properties depend on characteristics of amylose matrix, interactions between the dispersed and continuous phase and the rigidity of gelatinized granules (Eliasson, 1985). 
During storage of concentrated gels, stiffness increases due to rearrangement of starch chains. The short term development of gel structure was found to be dominated by the gelation of amylose within the continuous phase (Miles et al .,1985b). In starch gels, increase in crystallinity and gel stiffness over longer time periods was attributed to the reordering of amylopectin molecules which occurs at a much slower rate (Ring et al., 1987).

Starch gels develop 'B' type crystallinity on storage (Miles of al., 1985b: Russell, 1987; Van Soest et al., 1994) regardless of the initial crystalline pattern of the native starch. The intensity of the 'B' pattern has been shown to increase within the time of gel storage (Roulet et al., 1988; Eerlingen et al., 1994). Even though starch gels regain some of the structural order during retrogradation process (Miles et al., 1985b; Eerlingen et al., 1994; Van Soest et al., 1994), Keetels et al. (1996) showed that this order is different from the super-helical structure (Fig. 2.14) [Oostergetel \& van Bruggen, 1993] in the crystalline domains of native starch granules [since the size of semi crystalline clusters observed in retrograded starch gels was smaller $(5 \mathrm{~nm})$ than that in native starch $(9 \mathrm{~nm})]$. Based on these results, the above authors (Keetels et al., 1996) stated that the long range order in starch granules is not regained during starch retrogradation.

Non invasive methods such as FTIR and near infrared reflectance (NIR) spectroscopy have been used to monitor staling of bread (Wilson et al., 1991) and starch retrogradation (Van Soest et al., 1994, 1995). In bread crumbs, the increase in scattering of NIR radiation as the crumb structure changes during 
storage indicates the development of crystallinity in the amylopectin fraction (Wilson et al., 1991). FTIR measures short-range ordering in the gel system (Wilson et al., 1991). In the spectra, C-C and C-O regions $\left(1300-800 \mathrm{~cm}^{-1}\right)$ are sensitive to the retrogradation process (Van Soest et al., 1994, 1995). The spectra of potato starch geis $\left(10 \%\right.$ w/w) showed that a broad band at $1022 \mathrm{~cm}^{-1}$ resolved into three bands $\left(\sim 1053,1022\right.$ and $\left.1000 \mathrm{~cm}^{-1}\right)$ when the gels were stored for several weeks. The most pronounced changes of the spectrum occurred at 1000 (peak), 1035 (valley) and 1053 (peak) $\mathrm{cm}^{-1}$ (Van Soest et al., 1994). FTIR absorbance band at $1047 \mathrm{~cm}^{-1}$ is sensitive to the amount of ordered or crystalline starch, whereas the band at $1022 \mathrm{~cm}^{-1}$ is characteristic of amorphous starch (Van Soest et al., 1995). Based on these observations, the above authors proposed a multi-stage process for retrogradation of starch gels. These stages are listed below :

Stage 1 - conformational ordering : a) formation of double helices between amylose chains and / or between the outer branches of amylopectin chains, b) amylose chain aggregation and crystallization.

Stage 2 - onset of amylopectin helix aggregation and crystal growth.

Stage 3 - amylose aggregation and crystallization.

Stage 4 - phase separation of water (syneresis) due to excessive retrogradation. 


\subsubsection{Factors influencing retrogradation}

\subsection{Botanical source}

Russell (1987) studied the retrogradation of 4 starches with different amylose and amylopectin contents. DSC thermograms showed that after a given time, retrogradation endotherm for waxy maize starch was significantly greater than that for amylomaize starch while potato and wheat starches produced intermediate values. This suggests that the amylopectin fraction is responsible for the development of retrogradation endotherm. The initial development of modulus (Orford et al., 1987) observed for $30 \%$ starch gels from various botanical sources followed the order : pea > maize $>$ wheat $>$ potato. The dependence of initial rate of increase of the modulus on the botanical source can be accounted for by the different amounts of amylose that are solubilized during gelatinization. However, long term increase in modulus showed the order : pea > potato > maize > wheat. The above authors suggested that higher long term modulus increase in pea and potato was due to the their low lipid contents, which prevent amylopectin crystallization. Jacobson ot al. (1997) reported that retrogradation rates of $2 \%$ starch gels stored at $4^{\circ} \mathrm{C}$ for 56 days followed the order : wheat $\sim$ corn > rice tapioca, potato >> waxy maize. Upon storage, networked amylose transformed into a dense aggregated state, whereas amylopectin showed very little changes. Yuan \& Thompson (1998) studied the retrogradation of 3 waxy maize genotypes and reported that $\Delta \mathrm{H}_{\mathrm{R}}$ of duwx was greater than that of wx or wxshl. Storage modulus of duwx increased rapidly 
during the first 4 days of storage, whereas in wx and wxshl gels, the increase was gradual over 25 days of storage. The greater retrogradation tendency in duwx was attributed to the large proportion of DP 20-30 chains in amylopectin.

\subsection{Storage temperature}

Crystallization follows the three step mechanism of nucleation, propagation and crystal perfection (Wunderlich, 1976). Both nucleation and propagation (Fig. 2.27) depend exponentially on temperature, within the temperature range of glass transition $\left(T_{g}\right)$ and melting temperature $\left(T_{m}\right)$. Thus, nucleation rate increases with decrease in temperature down to $T_{g}$ while the propagation rate increases with increasing temperature up to the $T_{m}$ (Wunderlich, 1976). Several studies (Colwell et al., 1969; Feam, \& Russell, 1982; Jankowski, \& Rha, 1986) have shown that the rate limiting step for starch retrogradation is nucleation and the rate of retrogradation as well as the properties of retrograded starch gel depend on the storage temperature (Jankowski, \& Rha, 1986).

Differential thermal analysis of wheat starch gels stored at temperatures varying from -1 to $43^{\circ} \mathrm{C}$. (Colwell et al., 1969) demonstrated that instantaneous nucleation followed by rod-like crystal growth occurs throughout the temperature range. However, at elevated temperatures $\left(>10^{\circ} \mathrm{C}\right)$ more perfect crystallites are formed and the rate of aging is inversely related to storage temperature. Similar results were reported for potato starch gels stored at refrigeration temperature 
Fig. 2.27 Effect of storage temperature on crystallization of partially crystalline polymers (adapted from Wunderlich, 1976).

$\mathrm{Tg}$ - Glass transition temperature

Tm - Melting temperature

T - Temperature 


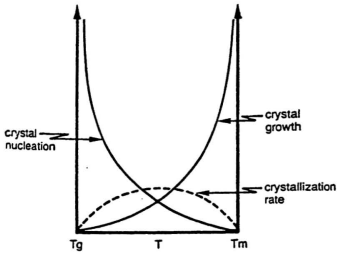


which formed less perfect crystallites than those stored at room temperature (Nakazawa et al.,1985).

Jankowski \& Rha (1986) observed that retrogradation endotherm of cooked wheat grains shifted to higher temperatures, when the storage temperature was increased from 4 to $20^{\circ} \mathrm{C}$. Similar findings were reported by

Jang \& Pyun (1997) for wheat starch gels (40-60\%) stored at 4 and $32^{\circ} \mathrm{C}$. A study with rice starch gets $(50 \%$ w/w) stored at different temperatures showed that, the extent of retrogradation followed the order : refrigerated > room temperature > frozen. Jacobson \& BeMiller (1998) showed that waxy maize starch gels $(2.5 \%)$ subjected to freeze thaw treatment showed a retrogradation rate which was inversely correlated to the rate of freezing. The peak temperature of retrogradation endotherm increased with increase in thawing temperature showing the formation of more perfect crystals.

\subsection{Moisture content of gels}

Gel moisture content was shown to influence crystal formation in starch gels. DSC showed that crystallization is greatest in gels with $50-60 \%$ starch (Longton \& LeGrys, 1981; Zeleznak \& Hoseney, 1986), whereas it does not occur in very dilute $(10 \%$ starch) or very concentrated $(80 \%$ starch) gels (Longton \& LeGrys, 1981). Orford et al. (1987) reported that, in maize starch gels stored for 7 days at $20^{\circ} \mathrm{C}$, development of shear modulus was rapid for gels having $30 \%$ and $40 \%$ starch, whereas the increase was very low for $20 \%$ gel. 


\subsection{Lipids}

Lipids and emulsifiers have been known to affect retrogradation, hence the texture of starch based products (Germani et al., 1983; Eliasson, 1985; Conde-Petit \& Escher, 1994). It appears that texture modification of starch is brought about by the formation of inclusion complexes particularly with monoacyl lipids (Eliasson, 1985; Conde-Petit \& Escher, 1994). However, it has also been suggested that reduced retrogradation may be due to hindered crystallization caused by the surface adhesion of the lipids on amylopectin chains (Van Lonkhuysen \& Blankestijn, 1974) or on the starch granule surface (Germani et al., 1983). In emulsifiers, the anti-firming effect is mainly caused by amylose complexation, which in turn weakens the cohesion between the amylopectin rich starch granules (Conde-Petit \& Escher, 1994). Decrease in granule swelling (Hoover \& Hadziyev, 1981), amylose leaching (Eliasson \& Krog. 1985), and changes in gel volume (Eliasson, 1985) have been shown to occur in the presence of MG and emulsifiers. The extent of the decrease depends on the nature of lipid (Hoover \& Hadziyev, 1981; Eliasson \& Ljunger, 1988). Germani et al. (1983) showed that the efficiency of lipid in decreasing retrogradation increased with decrease in chain length and increased degree of unsaturation. This is in agreement with the findings of Huang \& White (1993), who reported a greater inhibition of retrogradation in waxy corn gels with shorter MG chain length. Eliasson \& Ljunger (1988), demonstrated that MG hinder retrogradation more effectively than di- or triacylglycerols. The inhibition of retrogradation of 
rice starch gels in the presence of emulsifiers was shown to follow the order : glyceryl monopalmitate [GMP] > glyceryl monostearate [GMS], sucrose esters of palmitic acid, diacetyl tartaric acid, esters of MG > sucrose esters of stearic acid, sodium stearoyl-2- lactylate [SSL] (Miura et al., 1992). In 40\%(w/w) starch gels of potato, maize and wheat, the effect of added emulsifiers on inhibition of retrogradation (as measured by modulus of elasticity) followed the order : calcium stearoyl-lactyl-2-lactylate > GMS > lecithin (Conde-Petit \& Escher, 1994). Ward et al. (1994) showed (as monitored by DSC) that SSL did not significantly decrease the retrogradation of wheat and corn amylopectin gels. The above authors postulated that SSL affects the nature of crystallites formed but not the extent of crystallization.

\subsection{Sugars}

Effect of sugars on starch retrogradation is still in dispute. Several workers (Kohyama \& Nishinari, 1991; Katsuta et al., 1992a,b) support the idea that starch retards retrogradation whilst others (Chang \& Liu, 1991; Wang \& Jane, 1994) have provided evidence to the contrary. Germani et al. (1983) suggested that the mechanism of starch crystallization is instantaneous nucleation followed by a rod-like growth of crystals, regardless of the type of starch or the type of sugar used. However, Prokopowich \& Biliaderis (1995) demonstrated that the effect of sugars on retarding aging was solute specific (fructose accelerated retrogradation whereas maltotriose and ribose retarded the process), and more 
pronounced in starches with low amylose content compared to starches with high amylose. Slade \& Levin (1987) reported that the retrogradation of starch : sugar : water $(1: 1: 1)$ mixture stored at $25^{\circ} \mathrm{C}$ followed the order : fructose > mannose $>$ water alone $>$ glucose $>$ galactose $>$ maltose $>$ sucrose $>$ xylose $>$ lactose. The above authors suggested that sugars inhibit aging by decreasing the chain mobility and diffusion in the water / solute plasticized amylopectin matrix which raise the network $T_{g}$ of the starch gels. Thus, the rate of recrystallization at ambient temperatures (T) diminishes because of the lower temperature difference $\left(T-T_{g}\right)$. Using $X$-ray diffraction, Cairns et al. (1991) showed that xylose and ribose decreased crystallization of wheat starch gels with increasing sugar concentration. However, in gels containing fructose two effects were noted : addition of fructose led to both thermally reversible and irreversible crystallization upon storage. Similar findings were reported by Bliaderis \& Prokopowich (1994). Kohyama \& Nishinari (1991) observed that sucrose was more effective (sucrose > glucose > fructose) in inhibiting retrogradation of sweet potato starch gels. Among malto-oligosaccharides the effectiveness in reducing retrogradation followed the order : maltotriose > maltotetraose > branched oligosaccharides (Katsuta et al., 1992b Miura et al., 1992). It has been shown that hexoses (except galactose) are more effective than pentoses (Katsuta et al., 1992a) in retarding retrogradation while disaccharides are better than monosaccharides in stabilizing gel structures (Katsuta et al., 1992a; Miura et al., 1992). The above authors suggested that the 
ability of saccharides to stabilize starch-water systems might be influenced by the conformation of saccharides, whereas sugars with large number of equitorial hydroxyl groups are more effective in retarding crystallization. Moreover, based on the studies with a large number of polyhydroxy compounds, Biliaderis \& Prokopowich (1994) and Prokopowich \& Biliaderis (1995) postulated that the compatibility of sugar with the water structure (as governed by the stereochemistry of the main sugar conformers in solution) is important in controlling retrogradation kinetics. Seow et al. (1996) reported that recrystallization of rice starch gels increased to a maximum with increase in sucrose, xylose and arabinose concentration before decreasing with further addition of sucrose. However, fructose and maltose increased retrogradation over the concentration range of $0-100 \%$.

\subsection{Salts}

Clacco \& Fernandes (1979) showed that retrogradation rate of wheat starch gels increased by anions in the order : $\mathrm{r}<\mathrm{Br}<\mathrm{Cr}<\mathrm{F}$; and cations increased retrogradation in the order : $\mathrm{K}^{+}<\mathrm{Li}^{+}<\mathrm{Na}^{+}$. However, the crystallization mechanism (instantaneous nucleation followed by crystal growth) remained unchanged in the presence of anions or cations. Russell \& Oliver (1989) studied the effect of $\mathrm{NaCl}$ concentration $(0-4.43 \%)$ on aging of wheat starch gels by rheological and thermal measurements and observed that the increased salt concentration progressively increased the biphasic appearance of the 
retrogradation endotherm. This implies that increased salt concentration reduced re-ordering of the amylopectin fraction. Bello-Perez \& Paredes-Lopez (1995) showed that amaranth starch gels with $\mathrm{NaCl}$ produced an endotherm after storage of 4 weeks. The enthalpy of retrogradation decreased with increase in $\mathrm{NaCl}$ concentration from 0.05 to $0.2 \%$. However, waxy corn starch gels with $\mathrm{NaCl}$ did not produce a retrogradation endotherm within the 4 week storage period.

\subsection{Hydrocolloids}

Hydrocolloids have been known to affect the gelling behaviour of starches which is important in food product development and specifically modification of the texture and stability of formulated food systems (Christianson et al., 1981: Sajjan \& Rao, 1987). In general, hydrocolloids have been shown to accelerate gelling (Christianson et al., 1981; Alloncle \& Doublier, 1991). However, the mechanism by which hydrocolloids such as guar gum, xanthan gum, carageenan influence the gelation and crystallization mechanism is still in dispute (Eidam et al., 1995; Biliaderis et al., 1997).

\subsection{Physical modification}

Orford et al. (1993) investigated the retrogradation of extrusion-cooked waxy maize starch gels (15-50\%) stored at $1^{\circ} \mathrm{C}$ using DSC and gel firmness measurements. The results showed that extrusion cooked products formed thermoreversible gels, but caused less apparent changes in gelation kinetics. 
Retrogradation of defatted and heat-moisture treated pigeon pea starch gels stored at $-16^{\circ} \mathrm{C}$ was studied using freeze thaw stability measurements (Hoover et al., 1993). The results showed that both treatments decreased the symeresis of starch gels compared to the unmodified gels. Hoover et al. (1994) showed that defatting and heat-moisture treatment did not alter the onset time of the retrogradation endotherm (endotherm appeared after 3 days in native and treated starches) of wheat, potato and lentil starches. However, in oat starch the above treatments accelerated the appearance of endotherm (endotherm developed after 3 and 6 days respectively, for defatted and heat-moisture treated starches). Defatting increased the enthalpy of retrogradation $\left(\Delta \mathrm{H}_{R}\right)$ in all starches, whereas heat-moisture treatment increased $\Delta \mathrm{H}_{\mathrm{R}}$ in wheat, lentil and oat starches but decreased $\Delta \mathrm{H}_{\mathrm{R}}$ in potato starch. The gel strength of wheat and oat starches increased to a greater extent on defatting than on heat-moisture treatment, whereas in potato and lentil starches the increase was more pronounced on heat-moisture treatment. The above authors (Hoover et al., 1993. 1994) explained the changes based on the structural changes that occur within the granule during defatting and heat-moisture treatment. Durani \& Donald (1995) observed that amylopectin gels which were annealed (heating the sample at a temperature below its melting point) at a temperature below their melting point (after storage at $4^{\circ} \mathrm{C}$ ) melted at a higher temperature and over a narrower range than those which were not annealed. Furthermore, the meiting 
temperature range of these gels were affected by the length of time of annealing as well as the annealed temperature.

\subsection{Chemical modification}

Introduction of phosphate (Bohlin \& Eliasson, 1986), acetylated (Hoover \& Sosulski, 1985) and hydroxypropylated (Hoover ot al., 1988; Yook et al., 1993) groups into starch chains has been shown to influence starch retrogradation by retarding amylose and amylopectin chain aggregation during storage. Hoover ef al. (1988) reported that hydroxypropylated field pea starch showed less retrogradation during frozen storage compared to the unmodified starch. Takahashi et al. (1989) studied the gel properties of wheat and corn starches cross linked after acetylation and hydroxypropylation. Both cross linked acetylated and cross linked hydroxypropylated wheat and com starches showed a low gel firmness compared to their unmodified gels. Both modification methods reduced the gel firmness of wheat starch to a greater extent compared to modified corn starch. Furthermore, gel firmness decreased with increasing cross linking in both acetylated and hydroxypropylated wheat and corn starches. Freeze-thaw stability of hydroxypropylated field pea starches increased with increased substitution (MS 0.0 to 0.12 ). DSC showed that hydroxypropylation (Yook et al., 1993) of rice decreased retrogradation of cooked rice. 


\subsubsection{Starch digestibility : Action of porcine pancreatic $\alpha$-amylase}

The digestibility of starch by porcine pancreatic $\alpha$-amylase has been the subject of numerous investigations (Holm et al., 1983; Seneviratne \& Biliaderis, 1991; Cone \& Wolters, 1990). The action of porcine pancreatic $\alpha$-amylase on starch chains is known to occur by a multiple attack mechanism (Robyt \& French, 1970) in which once the enzyme forms an enzyme-polymer complex (Fig. 2.28a), the enzyme may catalyze the hydrolysis of several bonds before it dissociates. The direction of multiple attack is from reducing to non reducing end of the molecule (Robyt \& French, 1970). Being an endo-enzyme, porcine pancreatic $\alpha$-amylase randomly cleaves $\alpha-(1-4)$ glycosidic linkages of linear amylose producing mainly $G_{2}$ and $G_{3}$ and finally $G_{1}$ and $G_{2}$ after prolonged incubation with a large amount of enzyme (Robyt \& French, 1970). Porcine pancreatic $\alpha$-amylase consists of an active site with $5 \mathrm{D}$ - glucose sub sites (Robyt \& French, 1970) and a catalytic site consisting of carboxylate anion (nucleophile) and imidazolium cation [electrophile] (Hoover \& Sosulski, 1985). Porcine pancreatic $\alpha$-amylase shows a wide variation in in vitro digestibility of native, gelatinized and modified starches. This wide variation has been attributed to several factors : starch crystallinity (Hoover \& Sosuiski, 1985; Ring et al., 1988), amylose / amylopectin content (Atkins \& Kennedy, 1985), granule size (Cone, \& Wolters, 1990; Franco et al., 1992), starch-lipid interactions (Seneviratne \& Biliaderis, 1991; Holm et al., 1983), starch - protein 
Fig. 2.28 Starch hydrolysis by $\alpha$-amylase : mechanism

A) Enzyme - substrate complex formation and hydrolysis of $\alpha(1-4)$ glycosidic bonds via multiple attack mechanism (adapted from Banks \& Greenwood, 1975).

1) an internal segment of amylose is bound to the active site of the enzyme; hydrolytic scission occurs at catalytic site.

2) after hydrolytic scission, the fragment with non reducing end diffuses away, the remaining fragment is again bound to the enzyme.

3) the substrate rearranges itself and the second hydrolytic scission occurs.

B) Schematic representation of 'chair' to 'half chair' conformations of the Dglucosyl residues of starch molecules during hydrolysis by $\alpha$-amylase (Thoma, 1968). 

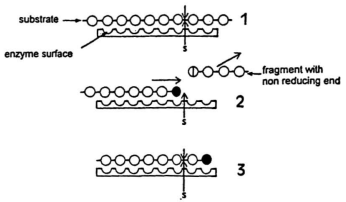

$\mathrm{S}=$ catalytic site

- reducing end

B

Chair form

Half chair form

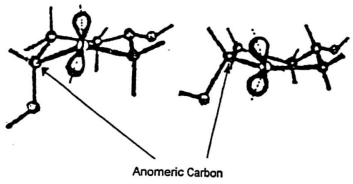


interactions (Wursch et al., 1986), starch modification (Wootton \& Chaudhry. 1981; Hoover et al., 1993; \& Hoover \& Manuel, 1996b), and retrograded starch (Jane \& Robyt, 1984; Kim et al., 1997).

The enzyme catalyzed hydrolysis of $\alpha-D$ glucose linkages has been shown to involve in an enzyme induced ring distortion of one of the D-glucosyt residues from the ${ }^{4} \mathrm{C}_{1}$ 'chair' conformation to 'half chair' conformation [Fig. 2.28b] (Thoma, 1968). This ring distortion decreases the enthalpy of activation and the susceptibility of the glucosyl residues to nucleophilic attack by functional groups on $\alpha$-amylase and water. Laszlo et al. (1978) have shown that ring distortion or a 'half chair' conformation is involved in transition state of $\alpha$ amylase.

Potato amylose complexed with lysolecithin and oleic acid showed reduced hydrolysis with porcine pancreatic $\alpha$-amylase (Holm et al., 1983). Eliasson \& Krog (1985) reported that amylose complexed with saturated MG was more resistant to enzymatic degradation than that of unsaturated MG. Seneviratne \& Biliaderis (1991) demonstrated that the rate and the extent of hydrolysis of helical inciusion complexes of amylose by porcine pancreatic $\alpha$ amylase and $\alpha$-amylase from Bacillus subtilis was higher in form I than form II amylose - lipid complexes. This was due to the fact that form I with less ordered structure (more open) in the solid state exhibits the highest susceptibility to $\alpha$-amylase. This suggests that the super molecular structure 
of $\mathrm{V}$ amylose complex influences the accessibility of $\alpha$-amylases to the solid substrate and thereby controls the digestion kinetics. Hoover \& Manuel (1996b) suggested that a conformational change during $\alpha$-amylase hydrolysis may be difficult for those amylose chains that are complexed by native lipids.

\subsection{Starch modification}

The physical properties of native starches and their colloidal sols produced on heating the suspension, limit the usefulness of starch in many commercial applications. Depending on the application, these drawbacks may include the lack of free flowing properties or water repellence of the starch granules, insolubility in cold water, excess or uncontrolled viscosity after cooking, cohesive or rubbery texture of the cooked starch, sensitivity to shear and $\mathrm{pH}$, the lack of clarity and tendency of becoming opaque geis when cooled (Wurzburg, 1987). Therefore, native starches are modified by physical, chemical or enzymatic processes to overcome one or more of these drawbacks, thus expanding the usefulness of starch in many applications.

\subsubsection{Physical modification of starch}

Physical modification of starch generally involves the simultaneous action

of factors such as temperature, moisture, pressure, and shear. The effect of 
these factors on starches causes two types of transformations : 1) modification of the physical structure with either the conversion or the complete disorganization of the granule and 2) modifications at the molecular level involving either degradation of macromolecules or of the monomers (Colonna et al., 1987). Physical modification methods which bring about these transformations include heat-moisture treatment, defatting (with various solvents), annealing, extrusion cooking and preglatinization.

\subsubsection{Heat-moisture treatment}

Physicochemical properties (swelling, amylose leaching, gelatinization, susceptibility to enzyme) of starches have been shown to change dramatically on heat-moisture treatment $\left(18-27 \%\right.$ moisture, $\left.100^{\circ} \mathrm{C}\right)$ [Sair, 1967; Kulp \& Lorenz, 1981; Hoover \& Vasanthan, 1994a,b]. The extent of these changes are highly influenced by starch source, moisture content, time and temperature of heatmoisture treatment (Lorenz \& Kulp, 1983; Stute, 1992; Hoover \& Vasanthan, 1994a,b; Franco et al., 1995). In general, physicochemical properties of tuber starches have been shown to approach those of untreated cereal starches after heat-moisture treatment (indicating structural changes within the starch granule). Physicochemical properties of tuber starches (potato, cassava) have been shown to improve on heat-moisture treatment, whereas in cereal starches (wheat, barley, triticale) they deteriorate after heat-moisture treatment (Lorenz \& Kulp. 1981). Kulp \& Lorenz (1981) reported that the disruption of granule 
structure on heat-moisture treatment is more evident in wheat than in potato starch. Donovan et al. (1983) postulated that the effect of heat-moisture treatment may be either due to new crystal formation or recrystallization and perfection of the crystalline regions of the starch granules. Hoover \& Vasanthan (1994a), Franco et al. (1995) and Hoover \& Manuel (1996a) have shown that the extent of starch chain associations within amorphous regions and the degree of crystalline order are changed on heat-moisture treatment of cereal, tuber and legume starches. The magnitude of these changes was found to be dependent upon the moisture content (during heat treatment) and the starch source. Several researchers have shown that the $X$-ray pattern of potato and yam starches (Hoover, \& Vasanthan, 1994a) change from a 'B' pattern to 'A+B' pattern on heat-moisture treatment . However, in cereal starches, the 'A' pattern remains unaltered on heat-moisture treatment (Lorenz \& Kulp, 1983; Stute 1992; Hoover \& Manuel, 1996a)

Imberty et al. (1991) have shown that in crystallites of both 'A' and ' $\mathrm{B}$ ' starches double helices are found in pairs and all chains are packed in parallel arrays. The pairing of double helices is the same in both polymorphs and corresponds to the interaction between double helices that have the lowest energy. Starches exhibiting 'A' and 'B' $\mathrm{X}$-ray patterns differ in their water content and the manner in which the pairs of double helices are packed within their respective crystals. In ' $\mathrm{B}$ ' starches there are 36 water molecules present in a channel in the centre of a hexagonal arrangement of six double helices, while in 
'A' starches there are only four water molecules between double helices (Imberty et al., 1991). Furthermore, the centre of ' $A$ ' starches is occupied by an amylosic helix rather than a column of water. It has been suggested that adjacent double helices within crystallites of ' $\mathrm{A}$ ' starches are mainly linked by direct hydrogen bonding (Leach et al., 1959; Imberty et al., 1991). However, in crystallites of 'B' starches, adjacent double helices are mainly linked by hydrated water bridges and to a limited extent by direct hydrogen bonding (Imberty et al., 1991). The transformation of the $X$-ray pattern from 'B' to 'A+B' is probably initiated by rupture of the hydrated water bridges which enables helices to rearrange themselves into a crystalline array that contains an amylosic helix in the central channel of the unit cell (Hoover \& Vasanthan, 1994a).

Hoover \& Vasanthan (1994a) observed that the crystallinity of tuber starches decreased, whereas it increased in wheat, lentil and oat starches after heat-moisture treatment. Hoover \& Manuel (1996a) observed increased crystallinity in normal, waxy, dull waxy and amylomaize $V$ starches after heatmoisture treatment (at $30 \%$ moisture).

Heat-moisture treatment of cereal, tuber and legume starches at varying moisture contents for different lengths of time have been shown to alter gelatinization transition temperatures (onset, mid point and conclusion) and the enthalpy of gelatinization (Lorenz \& Kulp, 1983; Stute; 1992; Hoover \& Vasanthan, 1994a; Hoover \& Manuel, 1996). The extent of these changes was found to be dependent upon the starch source and conditions of heat-moisture 
treatment. Reported results of studies on HMT $\left(95-110^{\circ} \mathrm{C}, 100 \%\right.$ relative humidity for $16 \mathrm{~h}$ ) of potato and com starches have shown that heat-moisture treatment increases transition temperatures (Sair, 1967). These results were confirmed by Kulp \& Lorenz (1981), Lorenz \& Kulp (1983) $\left[100^{\circ} \mathrm{C}, 18-27 \%\right.$ moisture, $16 \mathrm{~h}$ ], Donovan et al. (1983) [110 ${ }^{\circ} \mathrm{C}, 20 \%$ moisture, $\left.140-240 \mathrm{~min}\right]$, and Hoover \& Vasanthan (1994a) $\left[100^{\circ} \mathrm{C}, 30 \%\right.$ moisture, $\left.16 \mathrm{~h}\right]$ for wheat, barley, triticale, red millet, potato, arrowroot, and cassava starches. It was further shown that the gelatinization enthalpy decreases after heat-moisture treatment of potato starch (Donovan et al., 1983; Stute, 1992; Hoover \& Vasanthan, 1994a). Only a slight (Donovan et al., 1983) or no decrease (Stute, 1992; Hoover \& Vasanthan, 1994a; Hoover \& Manuel, 1996a) was observed after heat-moisture treatment of cereal starches. Possible explanations for the effect of heatmoisture treatment on gelatinization parameters include : 1) increase in interactions between amylose and amylopectin or lipids (Hoover \& Vasanthan, 1994a), resulting in a decrease in the destabilizing effect exerted by the amorphous regions on the melting of starch crystallites during gelatinization; 2) alteration of the interactions between crystallites and amorphous matrix (Stute. 1992); 3) changes in the stability of starch crystallites (Hoover \& Vasanthan, 1994a; Eerlingen et al., 1996) and 4) reorientation of double helices (Hoover \& Vasanthan, 1994a). 


\subsubsection{Defatting}

Defatting was shown to alter crystallinity, solubility, swelling, enzyme susceptibility, pasting and gelatinization parameters of cereal, tuber and legume starches. The extent of the above changes was influenced by the botanical source, nature and composition of the solvent, temperature and time of defatting, degree of associations between amylose and amylopectin chains in the native granule and on the lipid content (Takahashi \& Seib, 1988; Vasanthan \& Hoover, 1992a; Gibinski et al., 1993).

Takahashi \& Seib (1988) reported that defatting with methanol $\left(30^{\circ} \mathrm{C}\right)$ and $75 \%$ n-propanol (at $100^{\circ} \mathrm{C}$ ) removed $93 \%$ and $88 \%$ lipid from com starch respectively. However, for wheat starch the corresponding values were $78 \%$ and $97 \%$, respectively. lodine binding capacity $[\mathrm{IBC}]$ has been used to indicate the efficiency of lipid removal. The above authors noted that wheat and com starches showed the highest IBC after extraction with $75 \%$ n-propanol $\left(100^{\circ} \mathrm{C}\right)$. Vasanthan \& Hoover (1992b) reported that defatting with 1-propanol water 3:1 (v/v) [PW] removed almost all the lipids in wheat, corn, potato, lentil and cassava starches.

Defatting with $80 \%$ methanol was shown to produce unaltered $X$-ray patterns and decreased crystallinity in wheat and potato starches (Lorenz, \& Kulp, 1983). Vasanthan \& Hoover (1992a) observed an increase in crystallinity and a change in $X$-ray pattern from ' $B$ ' to 'A+B' on defatting potato starch with PW. However, in wheat, corn and cassava starche, the $\mathrm{X}$-ray pattern and 
crystallinity remained unchanged on deffatting. The change in X-ray pattern in potato starch was attributed (Vasanthan \& Hoover, 1992a) to clustering of the outer branched of amylopectin chains [resulting in the formation of a closed pack arrangement of double helices].

Swelling of cereal starches is primarily a property of amylopectin. Amylose and lipids act as diluents (Tester \& Morrison, 1990a). Partial removal of lipid in wheat starch (Tester \& Morrison, 1990a) with anhydrous methanol at $100^{\circ} \mathrm{C}$ increased swelling factor [SF]. The above authors suggested that the natural lipids in starch cause a substantial suppression of swelling in cereal starches. Defatting with $80 \%$ methanol increased the solubility and swelling power [SP] of wheat starch, whereas in potato starch, solubility decreased, while SP remained unaltered (Lorenz \& Kulp, 1983). Goshima and coworkers (1985) noted that in potato starch solubility and SP increased on defatting with $99 \%$ methanol. Vasanthan \& Hoover (1992a) and Hoover et al (1994) reported that defatting of potato, wheat, oat, corn, lentil and cassava starches with PW decreased SF of all starches. Gibinski et al. (1993) observed that defatting of oat starch varieties (Halny, Komes and Santor) with cold chloroform-methanol-water (3:2:1, v/v/v) or hot 1-propanol, increased the water binding capacity (cold extraction > hot extraction) and solubility (hot extraction $\gg$ cold extraction) of all three varieties. The above authors postulated that the increase in water binding capacity was probably due to the opening of the capillaries (which contained lipid micelles) on the surface of granules for water uptake after defatting. The increase in solubility 
was attributed to the damaged amylopectin shell of the granule by the joint action of alcohol and temperature (Gibinski $\theta t$ al., 1993).

Goshima et al. (1985) observed no significant changes in gelatinization temperatures when potato starch was defatted with $99 \%$ methanol. Similar observations have been reported for defatted wheat starch with $80 \%$ methanol (Lorenz \& Kulp, 1983) and defatted wheat and com starches with $75 \%$ ethanol (Takahashi \& Seib, 1988). In contrast, Vasanthan \& Hoover (1992a) noted that defatting with PW increased the gelatinization temperatures of potato and lentil starches, but caused no significant changes in corn, wheat and cassava starches.

Lipid removal from potato starch with $99 \%$ methanol (15h) reduced the pasting temperature, increased paste consistency, but did not alter thermal stability during the holding period at $95^{\circ} \mathrm{C}$ (Goshima et al., 1985). Reduced pasting temperature, increased paste consistency $(\mathrm{PC})$ and pasting peak were also observed in wheat and com starches when defatted with water saturated butanol $(5 \mathrm{~h})$ at $70^{\circ} \mathrm{C}$ or with $85 \%$ methanol $(72 \mathrm{~h})$ (Melvin, 1979). However. Takahashi \& Seib (1988) noted that lipid removal from wheat and com starches with $75 \%$ ethanol eliminated the pasting peak, decreased pasting temperature, paste consistency and set back. Vasanthan \& Hoover (1992a) reported that defatting of cassava, potato, com, wheat and lentil starches with PW eliminated pasting peaks of cereal starches and increased thermal stability and reduced paste consistency in all starches. 


\subsubsection{Chemical modification}

Chemically modified starches have become important functional ingredients in a wide range of food and non food industrial applications, because of their improved functional properties over unmodified starches. Table 2.8 shows some of the properties and applications of chemically modified starches. The concept of chemical derivatization of starch to improve its functional properties for specific purposes began in late 1930s. An important outcome of this aspect was the discovery of a practical way to chemically modify starch in an aqueous slurry maintaining the integrity of the granule and making it possible to

remove by-products by filtration and washing techniques. Alkaline catalyzed reactions of starch with alkylene oxide were carried out in solution or paste form. Kesler \& Hjermstad (1950) showed that the reaction could be carried out to obtain hydroxyalkyt starches with undamaged granules. This discovery was especially important in the food industry for development of new starch products with improved properties (Kesler \& Hjermstad, 1950).

Today, chemical derivatization of starch is commonly carried out using methods such as acid conversion, cross linking, esterification and etherification which include cationization, carboxymethylation, hydroxyalkylation [Fleche, 1985). Acid converted starches are produced by hydrolyzing (with acids such as hydrochloric or sulfuric) a concentrated starch slurry ( $-40 \%$ starch) after heating at a temperature below its gelatinization temperature. During the process, acid hydrolyzes glycosidic linkages, thus shortening the chain length. The resulting 
Table 2.8 Properties and applications of chemically modified starches

\begin{tabular}{|c|c|c|c|c|}
\hline $\begin{array}{l}\text { Modified } \\
\text { starch }\end{array}$ & Treatment & $\begin{array}{l}\text { Advantages over } \\
\text { unmodified starch }\end{array}$ & Food applications & Non food applications \\
\hline Oxidized & hypochlorite & $\begin{array}{l}\text { reduced set-back } \\
\text { increased paste clarity }\end{array}$ & thickeners, jellies & $\begin{array}{l}\text { paper, textile, } \\
\text { adhesives }\end{array}$ \\
\hline Hydroxypropyl & propylene oxide & $\begin{array}{l}\text { increased stability and } \\
\text { clarity }\end{array}$ & $\begin{array}{l}\text { pie fillings, salad } \\
\text { dressings }\end{array}$ & $\begin{array}{l}\text { textile and paper } \\
\text { industry }\end{array}$ \\
\hline Esterified & acetic anhydride & $\begin{array}{l}\text { reduced set-back } \\
\text { increased clarity }\end{array}$ & $\begin{array}{l}\text { instant foods, frozen } \\
\text { foods }\end{array}$ & $\begin{array}{l}\text { textile, paper, } \\
\text { packaging, film }\end{array}$ \\
\hline Cross linked & phosphorus oxychloride & $\begin{array}{l}\text { increased stability to } \mathrm{pH} \text {, } \\
\text { shear, heat and freeze } \\
\text { thaw treatment }\end{array}$ & $\begin{array}{l}\text { canned and frozen } \\
\text { foods }\end{array}$ & $\begin{array}{l}\text { paper, metal } \\
\text { sequestrants }\end{array}$ \\
\hline Acid converted & acid & $\begin{array}{l}\text { high gel viscosity } \\
\text { low hot paste viscosity }\end{array}$ & jellies, gums & laundry starch, textile \\
\hline Cationic & $\begin{array}{l}\text { reagents containing amino, } \\
\text { imino, ammonium, } \\
\text { sulfonium groups (e.g. 2- } \\
\text { diethylaminoethyl chloride }\end{array}$ & $\begin{array}{l}\text { improved clarity and } \\
\text { stability of dispersions } \\
\text { cold water swelling }\end{array}$ & not used & $\begin{array}{l}\text { paper, textile, } \\
\text { adhesives, detergents }\end{array}$ \\
\hline $\begin{array}{l}\text { Carboxy- } \\
\text { methylation }\end{array}$ & sodium monochloroacetate & high viscosity & thickening agents & paints, adhesives \\
\hline
\end{tabular}

Adapted from Solarek (1986); Galliard \& Bowler (1987). 
product shows reduced hot paste viscosity, higher fluidity and solubility in hot water (Wurzburg, 1987).

Cross linking leads to a more rigid macromolecular network inside the granule by formation of inter- or intramolecular chemical bridges through hydroxy-substituted phosphate groups between starch chains (Fleche, 1985). Cross linking reaction is carried out in an aqueous starch suspension at temperatures varying from room temperature to $-50^{\circ} \mathrm{C}$ in the presence of a cross linking agent (e.g. phosphorous oxychloride, epichlohydrin, adipic acid). Cross linked starches show a great resistance to thermomechanical shearing. They can maintain their granule integrity in the presence of water under conditions that would rupture or destroy granules of unmodified starch (Fleche, 1985; Wurzburg, 1987).

Starch esters are formed by reacting starch suspension with acid anhydrides (acetic anhydride, malic anhydride) or carboxylic acids (acetic acid, formic acid) under appropriate $\mathrm{pH}$ and temperature conditions (Fleche, 1985). For example, acid anhydrides give optimum starch ester yield at $\mathrm{pH} 8.0-8.5$ and $15-25^{\circ} \mathrm{C}$ (Wurzburg, 1987). Currently, starch acetates with low degree of substitution ( $>0.1)$ are commonly used in many food and industrial applications (Table 2.8) since the low substitution level maintains the granule structure throughout the derivatization process. This gives commercial products with high purity which is necessary in food and pharmaceutical applications (Jarowenko, 1987). Starch esters with malic acid (starch malates) show low gelatinization 
temperature and better water retention over unmodified starches (Jarowenko, 1987).

Cationic starches are produced by chemical reaction with reagents containing amino, imino, ammonium, and sulphonium groups which carry a positive charge (Solarek, 1987). Commercially significant derivatives of cationic starches include tertiary amino and quaternary ammonium starch ethers. Their affinity for negatively charged substrates make them useful in paper industry to improve sheet strength by fibre bonding through ionic or ionic and hydrogen bonding (Solarek, 1987). Cationic starches also show improved paste clarity, stability (resistant to retrogradation) and also high dispersibility and solubility which give cold water solubility (Paschall, 1967).

Carboxymethylation is carried out by reacting sodium monochloroacetate with starch (in an alkaline medium) in a solid phase or in a homogeneous paste. The reaction temperature of the medium is in the range of $40-50^{\circ} \mathrm{C}$. Anionic nature of the group $-\mathrm{O}-\mathrm{CH}_{2}-\mathrm{COO}^{+} \mathrm{Na}^{*}$ gives a polyelectrolyte behaviour and high viscosity to the starch (Fleche, 1985). Thus, carboxymethyl starches are used as a thickener in many applications (Table 2.8). At a higher degree of substitution $(\sim 0.1)$, carboxymethyl starch becomes highly water soluble, resulting in cold water soluble starches (Hofreiter, 1987). 


\subsubsection{Hydroxypropylation}

Among hydroxyalkyl starches (hydroxyethyl, hydroxypropyl etc.), hydroxypropyl starches are the commonly used hydroxyalkyl derivatives in food and industrial applications (Fleche, 1985). Hydroxypropylation has been shown to retard gelling and retrogradation tendency of starch, increase water holding capacity, improve freeze thaw stability of starch based products and reduce the tensile strength and increase elongation of starch films. Hydroxypropyl starch is produced by treating an aqueous starch suspension with propylene oxide at $38-40^{\circ} \mathrm{C}$, in the presence of $\mathrm{NaOH}$ as a catalyst. The reactive nature of propylene oxide is due to its highly strained epoxide ring (Tuschhoff, 1986). In the formation of hydroxypropyl starch derivatives, part of the hydroxyl groups of the glucose units are converted into -O-(2-hydroxypropyl) group. The extent of hydroxypropylation is expressed in terms of molar substitution, which is defined as the number of moles of substituent per anhydroglucose unit (Fig. 2.29). In early stages, MS was determined by colourimetric method (Johnson, 1969). In this method, the development of colour is based on the reaction of ninhydrin with allyl alcohol and enol form of propanol, which is released during acid digestion. Recently, use of new techniques such as FTIR, 'H NMR (Forrest, 1992; de Graaf et al., 1995) has been employed for the rapid acquisition of results.

Several researchers postulated that hydroxypropyl substitution reaction occurs primarily in the amorphous regions of the starch granule composed of 
Fig. 2.29 Glucose units substituted with hydroxypropyl groups $(X)$ showing different molar substitution (MS)levels. 

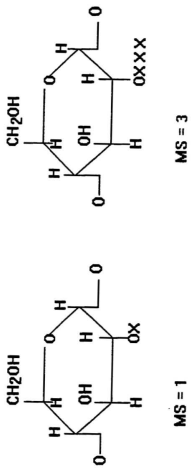
amylose and also in the intercrystalline areas of amylopectin (Hood, 1982; Blanshard, 1987). This is in agreement with the findings of Hood \& Mercier (1978) who showed that most of the hydroxypropyl groups in modified tapioca starch is located in the amorphous regions (which contain the majority of $\alpha-(1-6)$ branch points) whereas the more compact crystalline regions would be relatively impervious to the modifying agents, thus less affected. However, the loss of birefringence in chemically modified starch granules suggests that an irreversible change takes place within crystalline regions of the starch granule during modification (Hood \& Mercier, 1978). Kim et al. (1992) showed [using iodine affinity and Periodic Acid-Schiff's reagent staining] that hydroxypropylation mainly takes place in the central region of the potato starch granule. Furthermore; substitution at $\mathrm{C}-2$ and/or $\mathrm{C}-3$ on glucose units in hydroxypropylated potato starch has been reported to be $>90 \%$, whereas at C-6 it was $\sim 10 \%$ (Ostergard et al., 1988). Wootton \& Hariyadi (1992) reported that substitution at C-2 and C-6 was $94 \%$ and $6 \%$, respectively, but negligible at C-3. The distribution of substituents between C-2 and C- 6 was not affected by the level of MS or whether the parent starch was pre-gelatinized or not. However, the substitution at C-6 was affected by the starch type [wheat starch showed a higher substitution $(6 \%)$ at $\mathrm{C}-6$, than maize, waxy maize or high amylose maize (3\%)], but unaffected by the proportion of amylose in these starches (Wootton \& Hariyadi, 1992). 
Xu \& Seib (1997) studied (using 'H NMR) the distribution of hydroxypropyl substituents in alpha limit dextrins of 8 starches. The substitution at C-2, C-3 and C-6 were $67-78 \%, 15-29 \%$ and $3-17 \%$, respectively. The MS of these alpha limit dextrins was in the range of $0.05-0.23$, and correlated well with the values determined by the colorimetric procedure of Johnson (1969).

Hydroxypropylation was shown to alter physicochemical properties of starches (Wootton \& Manatsathit, 1984; Butler et al., 1986; Seow \& Thevamalar, 1993; Yeh \& Yeh, 1993; Yeh \& Jeng-Yune, 1996). Wootton \& Manatsathit (1984) showed (using DSC) that increase in MS of hydroxypropylated maize starches (MS 0-0.27) decreased gelatinization enthalpy $(\Delta H)$, onset $\left(T_{0}\right)$ and peak $\left(T_{p}\right)$ temperatures, whereas the gelatinization temperature range $\left(T_{c}-T_{0}\right)$ remained unaffected. Furthermore, a broadening of the endotherm was observed at MS > 0.1 (Wootton \& Manatsathit, 1984). At 1:1 starch : water ratio, hydroxypropylated rice starch showed a progressive shift of a biphasic gelatinization endotherm to lower temperatures and a decrease in $\Delta \mathrm{H}$ with increase in MS (0.0 to 0.1$)$ [Seow \& Thevamalar, 1993]. The above authors postulated that hydroxypropyl groups attached to starch molecules are primarily in the and behave as flexible side chains. The motion of these side chains creates a large amount of free volume, which may be considered as increasing internal plasticization and destabilization of amorphous regions of the granule. This destabilization of amorphous region lowers the glass transition and crystallite melting temperatures. At high MS levels, gelatinization temperature 
range increases, because derivatization has a greater effect on the amorphous region, and also because of the increased inhomogeneity within both amorphous and crystalline regions of the granule. The decrease in $\Delta \mathrm{H}$ was attributed to the increased disruption of local order in the amorphous regions (Seow \& Thevamalar, 1993). Decreased transition temperatures and $\Delta \mathrm{H}$ (compared to native starch) were also observed for hydroxypropylated rice starch by Yeh \& Yeh (1993) and Yeh \& Jeng-Yune (1996).

Butler et al. (1986) reported that swelling power (SP) of buffalo gourd root starch was unaltered on hydroxypropylation (MS $0.003-0.06$ ). In hydroxypropylated maize starches (MS $0-0.27$ ), SP increased at MS $>0.12$ (Wootton \& Manatsathit, 1983), whereas water binding capacity increased (MS > 0.1) following an initial decrease (MS $0.0-0.1$ ). The above authors suggested that the initial decrease in water binding capacity was due to the blocking of water binding sites by substituent groups, whereas further increase in water binding capacity was due the tendency of granule to swell at higher MS (Butler et al., 1986).

Hydroxypropylation (MS 0-0.12) of field pea starch exhibited reduced PT and increased viscosity at $95^{\circ} \mathrm{C}$ and $50^{\circ} \mathrm{C}$, with increasing MS levels (Hoover et al., 1988). Increased viscosity at $50^{\circ} \mathrm{C}$ implies reduced susceptibility to retrogradation (Butler ot al., 1986). Similar results have been reported for acetylated legume starches (Hoover \& Sosulski, 1985), hydroxypropylated buffalo gourd root starch (MS 0.00-0.06) and hydroxypropylated rice starch 
(Yeh \& Yeh, 1993). However, hydroxypropylation did not improve heat and shear resistance in rice starch (Yeh \& Yeh, 1993). Kim et al. (1992) observed altered pasting properties in hydroxypropylated potato starch and the changes were attributed to the decrease in associative forces within the starch granule. Increased paste clarity in hydroxypropyl starches with increasing MS has been noted by several researchers (Butler et al ., 1986; Hoover et al ., 1988; Rege \& Pai, 1996) and Rege \& Pai (1996) attributed the increased paste clarity to the increased amount of water bound to starch molecules.

Several researchers observed the decreased $\alpha$-amylolysis of raw starches with increase in MS (Leegwater \& Luten, 1971; Wootton \& Chaudhry. 1981; Mohd Azemi \& Wootton, 1984; Hoover et al., 1988). Mohd Azemi \& Wootton (1984), reported that susceptibility of raw waxy maize starch to $\alpha$ amylase attack showed a continuous drop with increase in MS, whereas normal maize and high amylose maize starches showed an initial decrease in hydrolysis followed by an increase in hydrolysis at higher substitution levels. Hydrolysis of gelatinized wheat [MS $0-0.17$ ] (Wootton \& Chaudhry, 1981), normal maize [MS 0- 0.12], waxy maize [MS 0-0.13] and high amylose maize [MS 0-0.08] (Mohd Azemi \& Wootton, 1984) starches decreased continuously with increase in MS. The decrease in hydrolysis in gelatinized starch and the initial decrease in hydrolysis in raw starch at low substitution levels was attributed to the presence of bulky hydroxypropyl groups on $\mathrm{C}-2$, which sterically hinder the action of catalytic carboxylate ion on the glycosidic bond (Mohd 
Azemi \& Wootton, 1984; 1995; Hoover et al., 1988), while the subsequent increase in hydrolysis in raw starch at higher MS (MS 0.12) was attributed to an increase in swelling power of the amorphous regions of the starch granules (Wootton \& Chaudhry, 1981; Mohd Azemi \& Wootton, 1985; Hoover et al., 1988) and also to granule disruption (Mohd Azemi \& Wootton, 1984). 


\section{CHAPTER 3}

\section{MATERIALS AND METHODS}

\subsection{Materials}

Potato tubers (Solanum tuberosum cv Russett Burbank) were purchased from the local market. Crystalline porcine pancreatic a-amylase (EC 3211) type 1A was obtained from Sigma Chemical Co (St. Louis, MO, USA). Other chemicals and solvents were analytical grade. Solvents were distilled from glass before use.

\subsection{Methods}

\subsubsection{Starch isolation and purification}

Potato tubers were divided into two lots representing the whole sample. Each lot was further subdivided into two parts and the starch was isolated according to the method of Hoover \& Hadziyev (1981) as follows :

The tubers were washed, diced, dipped in ice-cold water containing 100 ppm $\mathrm{NaHSO}_{3}$ and homogenized at low speed in a Waring blender. The slurry was squeezed through a 100-mesh polyester sieve cloth and the filtrate centrifuged at $700 \times \mathrm{g}$ for $15 \mathrm{~min}$. The supernatant and the amber-brown layer of protein atop the starch layer was removed. Further purification was achieved by repeated suspension in water, centrifugation and removal of contaminating proteins and cell debris. The purified starch was dried overnight at $30^{\circ} \mathrm{C}$ in a vacuum oven to a moisture content of $\sim 10 \%$. 


\subsubsection{Chemical composition of starch}

\subsubsection{Moisture content}

Quantitative estimation of moisture was performed according to standard AACC (1984) procedures. Preweighed (3-5g, db) samples of starch were dried in a forced air oven (Isotemp 614G. Fisher Scientific, Fair Lawn, NJ, USA) at $130^{\circ} \mathrm{C}$ for $1 \mathrm{~h}$. The samples were then removed and cooled in a desiccator. The moisture content was calculated as the percentage weight loss of the sample.

\subsubsection{Nitrogen content}

The nitrogen content was determined according to Micro Kjeldahl method. The samples $(0.3 \mathrm{~g} \mathrm{db})$ were weighed on nitrogen-free papers and placed in the digestion tubes of a Buchi 430 (Buchi Laboratoriums-Technik AG, Flawill / Schweiz) digester. The catalyst [2 Kjeltabs M pellets (Fisher Scientific, Fair Lawn, $\mathrm{NJ}$, USA)] and $20 \mathrm{ml}$ of concentrated $\mathrm{H}_{2} \mathrm{SO}_{4}$ acid were added and the samples were digested in the Buchi $\mathbf{4 3 0}$ digester until a clear yellow solution was obtained.

The digested samples were then cooled, diluted with $50 \mathrm{ml}$ of distilled water, $100 \mathrm{ml}$ of $40 \%(\mathrm{w} / \mathrm{w}) \mathrm{NaOH}$ were added, and the released ammonia was steam distilled into $50 \mathrm{ml}$ of $4 \% \mathrm{H}_{3} \mathrm{BO}_{3}$ containing 12 drops of end point indicator (N-point indicator, Sigma Chemical Co, St. Louis, MO, USA) using a Buchi 321 distillation unit until $150 \mathrm{ml}$ of distillate was collected. The amount of ammonia in the distillate was determined by titrating it against $0.05 \mathrm{~N} \mathrm{H}_{2} \mathrm{SO}_{4}$ (AACC, 1984). 
$\% \mathrm{~N}=$ (volume of acid - blank) $\times$ Normality of acid $\times 14.0067 \times 100$ sample weight $(\mathrm{mg})$

\subsubsection{Ash content}

Preweighed samples $(3-5 \mathrm{~g} . \mathrm{db})$ were transferred into clean, dry porcelain crucibles, charred using a flame and then placed in a pre-heated $\left(550^{\circ} \mathrm{C}\right)$ muffle furnace (Lab Heat, Blue Island, IL, USA) and left overnight until a gray ash was obtained. The samples were then cooled in a desiccator and weighed. The ash content was calculated as percentage weight of the remaining material (AACC. 1984).

\subsubsection{Lipid content}

Surface lipids were extracted at ambient temperature $\left(25-27^{\circ} \mathrm{C}\right)$ by mixing

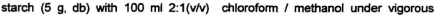
agitation in a wrist action shaker for $1 \mathrm{~h}$. Bound lipids were extracted using the residue left from surface lipid extraction. The residue was refluxed with 3:1 (v/v) n-propanol water in a soxhlet apparatus at $90-100^{\circ} \mathrm{C}$ for $7 \mathrm{~h}$. Total starch lipid was determined by hydrolysing starch $(2 \mathrm{~g}$, db) with $25 \mathrm{ml}$ of $24 \% \mathrm{HCl}$ at $70-$ $80^{\circ} \mathrm{C}$ for $30 \mathrm{~min}$ and extracting the hydrolysate 3 times with $\mathrm{n}$-hexane (Vasanthan \& Hoover, 1992b).

The crude lipid extracts from above extractions were purified by chloroform / methanol / water (1:2:0.8, v/v/v) and forming a biphasic system [chloroform / methanol / water (1:1:0.9 v/NN)] by addition of chloroform and water 
at room temperature (Bligh \& Dyer, 1959). The chloroform layer was then diluted with benzene and evaporated to dryness in a rotary evaporator (Rotavapor $\mathbf{R}$ 110, Brinkmann Instruments, Westbury, NJ, USA).

\subsubsection{Amylose content}

The apparent amylose content of native starch was determined by the method of Chrastil (1987). Starch samples (20 mg, db) were fully dispersed in $10 \mathrm{ml}$ of $0.5 \mathrm{~N} \mathrm{KOH}$ in cornical flasks. The dispersions were transferred into volumetric flasks and diluted with distilled water up to $100 \mathrm{ml}$. Aliquots $(10 \mathrm{ml})$ were neutralized with $5 \mathrm{ml}$ of $0.1 \mathrm{~N} \mathrm{HCl}$ and diluted to $50 \mathrm{ml}$ with distilled water. The sample preparation for determination of total (true) amylose content of starch samples was carried out following the same procedure, as above, after defatting starch with 3:1(v/V) n-propanol water for $7 \mathrm{~h}$.

\subsection{Chrastil's method of determination of amylose content}

Aliquots $(0.1 \mathrm{ml})$ of the neutralized and diluted solution were transferred into screw cap tubes containing $5 \mathrm{ml}$ of $0.5 \%$ trichloroacetic acid and then 0.05 $\mathrm{ml}$ of $0.01 \mathrm{~N} \mathrm{I}_{2}-\mathrm{Kl}$ solution was added. The tubes were allowed to stand for 30 min at room temperature for colour development. The absorbance of the blue colour was measured in a spectrophotometer (Novaspec Model 4049, LKB Biochrom, Cambridge, UK) at $620 \mathrm{~nm}$. The absorbance of the reaction blank with water was zero. The amylose content was determined using the formula : 
Absorbance $\times 32.5=\mathrm{mg}$ amylose / litre in curvette, and expressed as $\mathrm{mg}$ amylose per $100 \mathrm{mg}$ starch.

\subsubsection{Estimation of starch damage}

The starch damage was estimated following the standard AACC (1984) procedure. Starch samples $(1 \mathrm{~g}, \mathrm{db})$ were digested with fungal $\alpha$-amylase from Aspergillus oryzae (12500 Sigma units) having specific activity of 50-100 units $/ \mathrm{mg}$, in a water bath $\left(30^{\circ} \mathrm{C}\right)$ for $15 \mathrm{~min}$. At the end of incubation, the enzyme action was terminated by adding $3.68 \mathrm{~N} \mathrm{H}_{2} \mathrm{SO}_{4}(3 \mathrm{ml})$ and $12 \% \mathrm{Na}_{2} \mathrm{WO}_{4} \cdot 2 \cdot \mathrm{H}_{2} \mathrm{O}$ $(2 \mathrm{ml})$, respectively. The mixtures were allowed to stand for $2 \mathrm{~min}$ and then filtered through Whatman No 4 filter paper. The amount of reducing sugars in the filtrate was determined using the method of Bruner (1964). Aliquots (1 mi) of the filtrate were mixed with $2 \mathrm{ml}$ of chilled 3,5-dinitrosalicylic acid and diluted to $4 \mathrm{ml}$ with distilled water. The diluted samples were heated in a boiling water bath for 5 min. The reaction mixture was chilled, diluted with $8 \mathrm{ml}$ distilled water and the absorbance was measured at 540 and $590 \mathrm{~nm}$. A reagent blank was determined by the same procedure, but without starch.

The percentage starch damage was calculated as follows:

$\%$ starch damage $=[\mathrm{M} \times 1.64] /[\mathrm{W} \times 1.05] \times 100$ where $\mathrm{M}=\mathrm{mg}$ maltose equivalants in the digest; $\mathbf{W}=\mathrm{mg}$ starch (db); $1.05=$ molecular weight conversion of starch to maltose and $1.64=$ the reciprocal of the mean percentage maltose yield from gelatinized starch. The latter is an empirical 
factor which assumes that under the conditions of the experiment, the maximum hydrolysis is $61 \%$.

\subsubsection{Starch modification}

\subsubsection{Preparation of defatted potato starch}

Defatted starch was prepared by soxhlet extraction with $75 \%$ aqueous npropanol for $7 \mathrm{~h}$. The solvent was removed by vacuum evaporation and the starch was air dried to a moisture content of $\sim 10 \%$.

\subsubsection{Preparation of heat- moisture treated potato starch}

The heat-moisture treatment was essentially that of Sair (1964). Starch (15 g, db) was weighed into glass containers. Starch moisture content was brought to $30 \%$. The sealed samples (in glass jars) were heated in a forced air oven (Isotemp 615G, Fisher Scientific, Fair Lawn, NJ, USA) at $100^{\circ} \mathrm{C}$ for $16 \mathrm{~h}$. After cooling, the jars were opened and the starch samples were air-dried to a moisture content of $\sim 10 \%$.

\subsubsection{Preparation of hydroxypropylated potato starches}

Native, defatted and heat-moisture treated potato starch samples were converted into a range of hydroxypropyl derivatives according to the procedure of Leegwater \& Luten (1971). Samples $(200 \mathrm{~g}$, db) from each of the above starches were weighed into $600 \mathrm{ml}$ screw cap jars. Into each jar, a solution of 
$\mathrm{NaOH}(2.6 \mathrm{~g})$ and $\mathrm{Na}_{2} \mathrm{SO}_{4}(30 \mathrm{~g})$ in distilled water $(240 \mathrm{ml})$ was added at room temperature. The jars with samples were placed in a water bath at $40^{\circ} \mathrm{C}$ and propylene oxide $(0,4,10,20,30$ and $50 \mathrm{ml})$ was added and the suspensions thoroughly mixed and the jars closed. The reaction was continued at $40^{\circ} \mathrm{C}$ for 24 $\mathrm{h}$ with shaking. The starch suspensions were then adjusted to $\mathrm{pH} 5.5$ with dilute $\mathrm{H}_{2} \mathrm{SO}_{4}$ (1M). The starch cakes were washed with distilled water until negative to sulphate ions when tested with $\mathrm{BaCl}_{2}$. All hydroxypropylated suspensions were freeze dried until the moisture content was reduced to $10-12 \%$. Control potato starch was prepared by treatment of native, defatted and heat-moisture treated starches $\left(200 \mathrm{~g}\right.$, db) with distilled water containing $\mathrm{NaOH}(2.6 \mathrm{~g})$ and $\mathrm{Na}_{2} \mathrm{SO}_{4}(30$ g) but without addition of propylene oxide, according to the procedure for preparation of hydroxypropylated potato starch as described above.

\subsection{Determination of moiar substitution}

The hydroxypropyl content was determined by the spectrophotometric method of Johnson (1969). Modified starch samples (0.09-0.1 g db) were weighed into volumetric flasks, $25 \mathrm{ml}$ of $1 \mathrm{~N} \mathrm{H}_{2} \mathrm{SO}_{4}$ were added and heated in a boiling water bath until the samples dissolved. The samples were then cooled and diluted to $100 \mathrm{ml}$ with distilled water. Aliquots $(1 \mathrm{ml})$ of diluted samples were transferred into $25 \mathrm{ml}$ test tubes which were immersed in cold water, $8 \mathrm{ml}$ of concentrated $\mathrm{H}_{2} \mathrm{SO}_{4}$ was added, mixed and heated in a boiling water bath for 3 min. After the incubation, tubes chilled, $0.6 \mathrm{ml}$ of ninhydrin reagent [ $3 \%$ ninhydrin 
$\left(1,2,3\right.$ triketohydrin) in $\left.5 \% \mathrm{Na}_{2} \mathrm{~S}_{2} \mathrm{O}_{5}\right]$ was added and the tubes were placed in a water bath at $25^{\circ} \mathrm{C}$ for $100 \mathrm{~min}$. The solutions were then transferred into $25 \mathrm{ml}$ volumetric flasks, made up to the mark with concentrated $\mathrm{H}_{2} \mathrm{SO}_{4}$ and allowed to stand for $5 \mathrm{~min}$. The absorbance was measured at $595 \mathrm{~nm}$ using the solution without starch as the reference. A calibration curve was made using standard aqueous solutions containing 0-100 $\mathrm{pg}$ propylene glycol / $\mathrm{ml}$. The hydroxypropyl content was calculated and expressed in terms of molar substitution.

$\%$ of hydroxypropyl groups $=\mu$ g propylene glycol $\times 0.7763 \times 100 \times 100$ $10^{6} \times$ weight of starch $(\mathrm{g}, \mathrm{db})$

Molar substitution $=\%$ hydroxypropyl groups $\times 162.14$ $59.08 \times(100-\%$ hydroxypropyl groups $)$

A factor of 0.7763 was used to convert $\mu \mathrm{g}$ of propylene glycol to $\mu \mathrm{g}$ of hydroxypropyl groups (Johnson, 1969). $59.08=$ molecular weight of hydroxypropyl group, 162.14 = molecular weight of anhydroglucose unit.

Starch $-\mathrm{OH}+\mathrm{H}_{2} \mathrm{C} \underset{\mathrm{NaOH}, \mathrm{Na}_{2} \mathrm{SO}_{4}}{\stackrel{40^{\circ} \mathrm{CHCH}}{\longrightarrow}} \stackrel{\mathrm{OH}}{\longrightarrow}$ 


\subsubsection{Determination of physicochemical properties}

\subsubsection{Scanning electron microscopy (SEM)}

Granule morphology of native, defatted and heat-moisture treated starches were studied by SEM. Starch samples were mounted on circular aluminum stubs with double-sided sticky tape and then coated with $20 \mathrm{~nm}$ gold and examined and photographed in a Hitachi (S570) scanning electron microscope (Nissei Sangyo inc., Rexdale, ON, Canada) at an accelerating potential of $20 \mathrm{kV}$.

\subsubsection{X-ray diffraction}

X-ray diffractograms of starches were obtained with a Rigaku RU 200R Xray diffractometer (Rigaku-Denki Co., Tokyo, Japan) with a chart speed of 20 $\mathrm{mm} / \mathrm{min}$. The starch powder was scanned through the $2 \theta$ range of $3-35^{\circ}$. Diffractograms were obtained using $\mathrm{Cu}-\mathrm{K} \alpha$ radiation detector with a nickel filter and a scintillation counter operating under the following conditions : $40 \mathrm{kV}, 50$ $\mathrm{mA}, 1^{\circ} / 1^{\circ}$ divergence silt / scattering slit, $0.30 \mathrm{~mm}$ receiving slit, $1 \mathrm{~s}$ time constant and scanning rate of $3^{\circ} / \mathrm{min}$. The results were analyzed using the software Jade (version 2.1). 


\subsubsection{Differential scanning calorimetry (DSC)}

DSC measurements on native, defatted and heat-moisture treated starches were carried out using a Perkin-Elmer DSC-2 (Norwalk, CT, USA) Differential scanning caiorimeter with a thermal analysis data station. Water (8.0 $\mu$ ) was added with a microsyringe to starch $(2.5 \mathrm{mg})$ in DSC pans which were then sealed, reweighed and kept overnight at room temperature. The scanning temperature range and heating rate, were $20-120^{\circ} \mathrm{C}$ and $10^{\circ} \mathrm{Cmin}^{-1}$, respectively. The thermogram was recorded with water as a reference.

The transition temperatures reported are the onset $\left(T_{0}\right)$, peak $\left(T_{p}\right)$ and conclusion $\left(T_{c}\right)$ of the gelatinization endotherm. Indium was used for calibration. The enthalpy of gelatinization $(\Delta \mathrm{H})$ was estimated by integrating the area between the thermogram and the base line under the peak and expressed as joules per unit weight of dry $\operatorname{starch}(\mathrm{J} / \mathrm{g})$.

\subsubsection{Swelling factor (SF)}

The SF of the starches when heated to $60-90^{\circ} \mathrm{C}$ in excess water was determined according to the method of Tester and Morrison (1990a). Starch samples (50 mg. db) were weighed into screw cap tubes, $5 \mathrm{ml}$ water were added and heated in a shaking water bath at the appropriate temperature for $30 \mathrm{~min}$. The tubes were then cooled to $20^{\circ} \mathrm{C}, 0.5 \mathrm{ml}$ of blue dextran (Pharmacia, $\mathrm{M}_{\mathrm{r}}$ $2 \times 10^{6}, 5 \mathrm{mg} / \mathrm{ml}$ ) was added and mixed the contents by inverting the tubes. The tubes were then centrifuged at $1500 \times \mathrm{xg}$ for $5 \mathrm{~min}$ and the absorbance of the 
supernatant was read at $620 \mathrm{~nm}$ using a spectrophotometer (Novospec Model 4049, LKB Biochrom, Cambridge, UK). The absorbance of the reference which contained no starch was also measured at $620 \mathrm{~nm}$.

Calculation of SF was based on starch weight corrected to $10 \%$ moisture, assuming a density of $1.4 \mathrm{~g} / \mathrm{ml}$.

Free or interstitial plus supernatant water (FW) is given by

$F W=5.5\left(A_{r} / A_{s}\right)-0.5$

$A_{r}$ and As represent the absorbance of the reference and sample respectively.

The initial volume of the starch $\left(V_{0}\right)$ of weight $W$ (in $\mathrm{mg}$ ) is

$V_{0}=W / 1400$

and the volume of the absorbed intragranular water $\left(V_{1}\right)$ is thus

$V_{1}=5.0-F W$

Hence, the volume of the swollen starch granules $\left(V_{2}\right)$ is

$V_{2}=V_{0}+V_{1}$ and

$\mathrm{SF}=\mathrm{V}_{\mathbf{z}} / \mathrm{V}_{\mathrm{o}}$

This can also be expressed by the single equation

$\left.S F=1+\left\{(7700 / W) \times\left[\left(A_{s}-A_{s}\right) / A_{s}\right)\right]\right\}$

This method measures only intragranular water and hence the true SF at a given temperature. The SF is reported as the ratio of the volume of swollen starch granule to the volume of the dry starch. 


\subsubsection{Extent of amylose leaching}

Starch $(20 \mathrm{mg}, \mathrm{db})$ was heated in water $(10 \mathrm{ml})$ in volume calibrated sealed tubes $\left(50-90^{\circ} \mathrm{C}\right)$ for $30 \mathrm{~min}$. The tubes were then cooled to $25^{\circ} \mathrm{C}$ and centrifuged at $2000 \mathrm{~g}$ for $10 \mathrm{~min}$. The supernatant liquid $(0.1 \mathrm{ml})$ was withdrawn and its amylose content determined by the method of Chrastil (1987). Percentage amylose leaching was expressed as $\mathrm{mg}$ amylose leached per $100 \mathrm{~g}$ starch.

\subsubsection{Brabender viscosity measurements (Pasting properties)}

A Brabender viscoamylograph, Model VA-V (C.W.Brabender Instruments, Hackensack, NJ, USA) equipped with a $700 \mathrm{~cm} . \mathrm{g}$ cartridge was used to study pasting properties of starch slurries at a concentration of $6 \%(w / v)$ and $\mathrm{pH} 5.5$. The starch dispersions were stirred at $75 \mathrm{mpm}$ and heated at a rate of $1.5^{\circ} \mathrm{Cmin}^{-1}$ to $95^{\circ} \mathrm{C}$, kept at this temperature for $30 \mathrm{~min}$, and cooled to $50^{\circ} \mathrm{C}$. The viscosity was expressed in terms of Brabender units (BU) and the pasting temperature was defined as the temperature at which the viscosity showed an increase in 10 $\mathrm{BU}$ in the heating cycle. 


\subsubsection{Starch digestibility}

\subsubsection{Preparation of defatted potato starch for enzyme hydrolysis}

Defatted starch was prepared by soxhlet extraction with $75 \%$ aqueous $n-$ propanol for $1,2,4,7,9$ and $12 \mathrm{~h}$. The solvent was removed by vacuum evaporation and the starch was air dried to a moisture content of $\sim 10 \%$.

3.2.5.2 Preparation of heat-moisture treated potato starch for enzyme hydrolysis

The heat-moisture treatment was essentially that of Sair (1964). Starch (15 $\mathrm{g}$ dry basis) was weighed into glass containers. Starch moisture content was brought to $30 \%$. The sealed samples (in glass jars) were heated in a forced air oven (Isotemp 614G, Fisher Scientific, Fair Lawn, NJ, USA) at $100^{\circ} \mathrm{C}$ for $1,3,6$, 8, 10, 16 and $30 \mathrm{~h}$. After cooling the jars were opened and the starch samples air-dried to a moisture content $\sim \mathbf{1 0} \%$.

\subsubsection{Scanning electron microscopy of enzyme hydrolyzed starches}

Granule morphology of native, defatted (75\% n-propanol for $7 \mathrm{~h}$ ) and heat-moisture treated $\left(30 \%\right.$ moisture, $\left.100^{\circ} \mathrm{C}, 16 \mathrm{~h}\right)$ starches [before and after $\alpha$ amylase hydrolysis (72 h)] were studied by using a Hitachi (S570) scanning electron microscope (Nissei Sangyo Inc., Rexdale, ON, Canada) under the operating conditions mentioned before. 


\subsubsection{Enzymatic hydrolysis}

The extent of hydrolysis was determined using a crystalline suspension of porcine pancreatic $\alpha$-amylase in $2.9 \mathrm{M}$ saturated sodium chloride containing 3 $\mathrm{mM}$ calcium chloride in which the concentration of $\alpha$ - amylase was $30 \mathrm{mg} / \mathrm{ml}$ and the specific activity was 790 units per $\mathrm{mg}$ of protein. One unit was defined as the $\alpha$-amylase activity which liberates $1 \mathrm{mg}$ maltose in $3 \mathrm{~min}$ at $20^{\circ} \mathrm{C}$ at $\mathrm{pH} 6.9$. The procedure was essentially that of Knutson et al. (1982). Starch (100 mg. db) was suspended in distilled water $(25 \mathrm{ml})$ and $5 \mathrm{ml}$ aliquots were placed in a constant temperature water bath at $37^{\circ} \mathrm{C}$. Then $4.0 \mathrm{ml}$ of $0.1 \mathrm{M}$ phosphate buffer (pH 6.9) containing $0.006 \mathrm{M} \mathrm{NaCl}$ were added to the slurry. The mixture was gently stirred before adding $\alpha$-amylase suspension (12 units / mg starch). The reaction mixtures were shaken by hand in constant time periods to resuspend the deposited granules. Then $1 \mathrm{ml}$ aliquots were removed at specific time intervals, pipetted into $0.2 \mathrm{ml}$ of $95 \%$ ethanol, and centrifuged $(3000 \mathrm{~g})$. Aliquots of the supernatant were analyzed for soluble carbohydrate

(Bruner, 1964). Percentage hydrolysis was calculated as the amount (mg) of maltose released per $100 \mathrm{mg}$ of dry starch. Controls without enzyme, but subjected to the above experimental conditions, were run concurrently. 


\subsubsection{Starch retrogradation}

\subsubsection{Sample preparation for Turbidity measurements}

A $2 \%$ aqueous suspension of potato starch (native, defatted and heatmoisture treated), near neutral $\mathrm{pH}$, was heated in a boiling water bath for $1 \mathrm{~h}$ under constant stirring. After the suspension was cooled for $1 \mathrm{~h}$ at $25^{\circ} \mathrm{C}$, the turbidity was determined by measuring absorbance at $640 \mathrm{~nm}$ against a water blank with a Shimadzu UV-visible spectrophotometer (UV-260, Shimudzu Corporation, Kyoto, Japan). The development of turbidity was monitored by storing samples for 1 day at $4{ }^{\circ} \mathrm{C}$ followed by $2-35$ days $40^{\circ} \mathrm{C}$. This sequential incubation at $4^{\circ} \mathrm{C}$ and $40^{\circ} \mathrm{C}$ was applied to obtain extensive retrogradation in a short time by favouring nucleation (formation of crystal nuclei) at $4^{\circ} \mathrm{C}$ and propagation (growth of crystallites from the nuclei formed) of starch crystallites at $40^{\circ} \mathrm{C}$ (Wunderlich, 1976).

\subsubsection{Gel preparation for X-ray diffraction}

Gels were prepared (with minor modifications) as described by Krusi \& Neukom (1984). A $3 \%(w / v)$ potato starch gel was prepared by heating the suspension under gentle stirring for $15 \mathrm{~min}$ in a boiling water bath. After cooling to $30^{\circ} \mathrm{C}$, sufficient starch was added to obtain suspension with $40 \%$ (w/v) dry matter. These suspensions were then homogenized for $2 \mathrm{~min}$ at $8000 \mathrm{rm}$ and then heated in a forced air oven (Isotemp 614G, Fisher Scientific, Fair Lawn, NJ, 
USA) at $110^{\circ} \mathrm{C}$ for $2 \mathrm{~h}$. After cooling, the gels formed were stored at $4^{\circ} \mathrm{C}$ for 1 day followed by 29 days at $40^{\circ} \mathrm{C}$.

\subsection{Gel powder preparation for $X$-ray diffraction}

The procedure (with minor modifications) of Routlet et al. (1988) was used to convert freshly gelatinized and stored gels to a powder prior to examination by $X$-ray diffraction. The gels were rinsed with water, cut into small pieces and mixed with $100 \mathrm{ml}$ acetone. After homogenization using a polytron (T25 S-1, IKA works Inc., Cincinnati, OH, USA) the mixture was left to settle (for 5 min) and then decanted. The liquid was discarded and the rest was transferred to screw cap tubes. Acetone was again added, the mixture centrifuged $(3000 \mathrm{~g})$ and the supernatant discarded. The procedure was repeated three times and the remaining mass was then freeze dried.

$X$-ray diffractograms of gel powders were obtained with a Rigaku RU 200R X-ray diffractometer under the same conditions mentioned previously.

\subsubsection{Scanning electron microscopy of retrograded starch gels}

The specimen preparation of freshly gelatinized and stored ( 1 day at $4^{\circ} \mathrm{C}$ ) potato starch gels for SEM was carried out as follows : The starches $(2 \%$, w/V) were gelatinized under the conditions described for turbidity measurements and then stored for 1 day at $4^{\circ} \mathrm{C}$. The gels were then freeze dried and the samples examined and photographed in a Hitachi (S570) scanning electron microscope 
(Nissei Sangyo Inc., Rexdale, ON, Canada). The SEM operating conditions were carried out as outlined before.

\subsubsection{Differential scanning calorimetry of retrograded starches}

Thermal transitions of retrograded starches were investigated using a Perkin-Elmer DSC-2 (Norwalk, CT, USA) differential scanning calorimeter equipped with a thermal analysis data station. Water $(3 \mu)$ was added, with a microsyringe to starch $(3 \mathrm{mg})$ in DSC pans, which were then sealed, reweighed and kept for $1 \mathrm{~h}$ at room temperature. The scanning temperature range and the heating rate were $20-120^{\circ} \mathrm{C}$ and $10^{\circ} \mathrm{C} / \mathrm{min}$, respectively. The heated pans were then cooled to room temperature and stored for 1 day at $4^{\circ} \mathrm{C}$ foliowed by $2-7$ days at $40^{\circ} \mathrm{C}$. After this time period, the pans were left to equilibrate for $1 \mathrm{~h}$ at room temperature and then scanned under the same previous conditions. In all measurements an empty pan was used as reference and experiments were repeated at least thrice.

The transition temperatures reported are the onset $\left(T_{0}\right)$, peak $\left(T_{p}\right)$ and conclusion $\left(T_{c}\right)$ of the retrogradation endotherm. The enthalpy of retrogradation $\left(\Delta \mathrm{H}_{R}\right)$ was estimated by integrating the area between the thermogram and the baseline under the peak and expressed as joules per unit weight of dry starch. 


\subsubsection{Enzymatic hydrolysis of retrograded starches}

The reactivity of porcine pancreatic $\alpha$-amylase towards freshly gelatinized and retrograded potato starches was determined as follows : starch samples were dispersed in distilled water to make a $2 \%$ suspension. The dispersions were shaken while heated at $100^{\circ} \mathrm{C}$ for $1 \mathrm{~h}$. The gelatinized starches were cooled to $30^{\circ} \mathrm{C}$ and then stored at $4^{\circ} \mathrm{C}$ for 1 day, followed by at $40^{\circ} \mathrm{C}$ for 14 days. At the end of the storage period, the retrograded gels were freeze dried and converted to powders prior to enzyme hydrolysis.

The extent of hydrolysis of the freshly gelatinized and retrograded starches was determined using a crystalline suspension of porcine pancreatic $\alpha$ amylase in $2.9 \mathrm{M}$ saturated sodium chloride containing $3 \mathrm{mM}$ calcium chloride in which the concentration of $\alpha$ - amylase was $30 \mathrm{mg} / \mathrm{ml}$ and the specific activity was 790 units/mg of protein. One unit was defined as the $\alpha$-amylase activity which liberates $1 \mathrm{mg}$ maltose in $3 \mathrm{~min}$ at $20^{\circ} \mathrm{C}$ at $\mathrm{pH} \mathrm{6.9}$. The details of the procedure have been outlined before.

\subsubsection{Statistical analysis}

All experiments were done in triplicate. Analysis of variance was performed using Minitab statistical package (Minitab Inc., 1991). Duncan's new multiple range test was utilized for comparison among means. 


\section{CHAPTER 4}

\section{RESULTS AND DISCUSSION}

\subsection{CHEMICAL COMPOSITION OF NATIVE POTATO STARCH}

Data on the composition of isolated potato starch are presented in Table 4.1. The chemical composition showed that the starch contained $0.37 \%$ ash and $0.01 \%$ nitrogen. The nitrogen in isolated starch may come from the internal proteins (residual material from lipid-protein membranes of the original amyloplast or of membrane-bound starch synthesizing systems employed during development) [Galliard,1983], phospholipids containing ethanolamine, choline or endosperm storage proteins (Morrison, 1981). The purity of the starch was judged on the basis of composition and microscopic observations. The low values of nitrogen and ash indicated that the isolated potato starch is of high purity. Total lipids (obtained by acid hydrolysis) in potato starch $(0.12 \%)$ represent free and bound starch lipids. The free lipids (obtained by extraction with $\mathrm{CHCl}_{3}-\mathrm{CH}_{3} \mathrm{OH}$ ) amounted to $0.03 \%$, while the corresponding values for bound lipids (obtained by extraction of the $\mathrm{CHCl}_{3}-\mathrm{CH}_{3} \mathrm{OH}$ residue with n-propanol water) was $0.09 \%$. These values are in agreement with those reported by Vasanthan \& Hoover (1992b) for potato starch obtained from Sigma Co (St. Louis, MO, USA). The difference between total and apparent amylose contents indicates the amount of amylose complexed with lipid. According to the results

obtained, the total amylose content was $25.6 \%$ of which $16.8 \%$ was complexed by native lipids (Table 4.1 ). 
Characteristics

Mosture

Asth

Nitrogen

Lipid

Solvent extracted

chloroform-methanol ${ }^{2}$

n-propanol-water

Acid hydrolysed

Amylose content

total

apparent

Amyiose complexed by lipids?

Starch damage
Composition $^{1}(\%)$

$13.4 \pm 0.0$

$0.37 \pm 0.01$

$0.01 \pm 0.01$
$0.03 \pm 0.01$

$0.09 \pm 0.00$

$0.12 \pm 0.01$

$25.6 \pm 0.9$

$21.3 \pm 0.6$

$16.8+0.6$

$0.3 \pm 0.0$

All data reported on dry basis and represent the mean of 3 determinations.

Lipid obtained from native starch by chloroform-methanol $2: 1\left(\mathrm{~V} / \mathrm{V}\right.$ ) at $25^{\circ} \mathrm{C}$ (mainly unbound lipids).

Lipid extracted by hot n-propanol water $(3: 1, v / v)$ from the residue left after chloroform-methanol extraction (mainly bound lipids). Lipid obtained by acid hydrolysis $(24 \% \mathrm{HCl}$ ) of native starch (total lipids).

Total amylose determined by iodine binding after removal of free and bound lipids. Apparent amylose determined by iodine binding without removal of free and bound lipids

Total amylose - apparent amylose $\times 100$

Total amylose 


\subsection{EFFECT OF DEFATTING AND HEAT-MOISTURE TREATMENT ON THE}

STRUCTURE AND PHYSICOCHEMICAL PREOPERTIES OF NATIVE POTATO STARCH

\subsubsection{Morphological granular characteristics}

Native potato starch granules were mainly oval to elliptical in shape. The surfaces appeared to be smooth (devoid of cracks or other damages) when viewed under the scanning electron microscope (Fig. 4.1a). Neither defatting (Fig. 4.1b) nor heat-moisture treatment (Fig. 4.1c) altered the shapes or the surface characteristics of the starch granules.

\subsubsection{X-ray diffraction}

The $X$ - ray patterns and $X$-ray intensities of native, defatted and heatmoisture treated potato starches are presented in Fig. 4.2. and Table 4.2. Native potato starch (Fig. 4.2a) exhibited the characteristic 'B' type X-ray pattern of tuber starches with peaks centered at $15.8,5.2,4.5,3.9$ and $3.8 \AA$. Defatting (Fig. 4.2b) resulted in an increase in intensity of the peak centred at $3.9 \AA$, a decrease in intensity of the peaks at 15.8 and $5.2 \mathrm{~A}$, elimination of the peak at $4.5 \mathrm{~A}$ and in the appearance of four additional peaks at $2.9,3.3,3.8$ and $4.6 \AA$ (Table 4.2). Furthermore, defatting changed the $X$-ray pattem from ' $B$ ' to ' $A+B$ ' (the ' $A$ ' pattern is characteristic of cereal starches) with intensities at d-spacings of 5.8, 5.2 and $3.8 \AA$ (Fig. 4.2). Heat-moisture treatment at $100^{\circ} \mathrm{C}, 30 \%$ moisture 
Fig. 4.1 Scanning electron micrographs of native $(A)$, defatted $(B)$ and heatmoisture treated $(\mathrm{C})$ potato starch granules. 


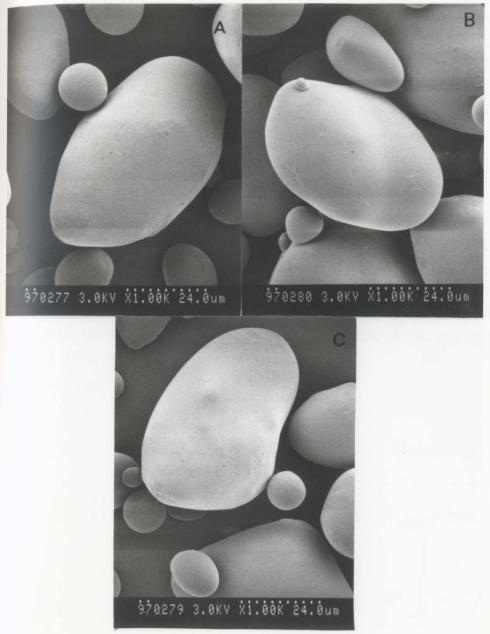


Fig. 4.2 X-ray diffraction patterns of native, defatted and heat-moisture treated (HMT) potato starches. (A) native [moisture content (MC) 9.5\%], (B) defatted (MC 9.3\%), (C) HMT $\left[100^{\circ} \mathrm{C}, 30 \%\right.$ moisture, $\left.16 \mathrm{~h}\right]$ (MC 9.3\%) and (D) HMT $\left[110^{\circ} \mathrm{C}, 30 \%\right.$ moisture, $\left.16 \mathrm{~h}\right](\mathrm{MC} 9.3 \%)$. 


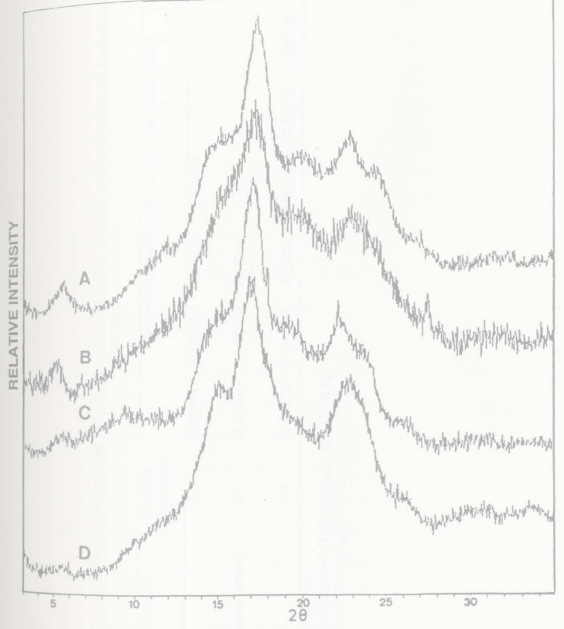




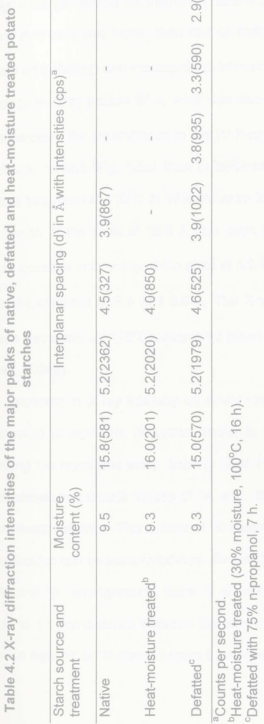


for $16 \mathrm{~h}$ (Fig. 4.2c) decreased the intensity (Table 4.2) of the peaks at 15.8 (the extent of this decrease was higher than that on defatting) and $5.2 \mathrm{~A}$ (similar to that observed on defatting) and increased the intensity (Table 4.2) of the peak at 4.5 A. A change in X-ray pattern ' $B$ ' $\rightarrow$ ' $A+B$ ' was also observed on heat-moisture treatment. However, the resemblance to the ' $A$ ' $X$-ray pattern was more marked in heat-moisture treated (Fig. 4.2c) than in defatted potato starch (Fig. 4.2b). Heat-moisture treatment at $110^{\circ} \mathrm{C}$ at $30 \%$ moisture for $16 \mathrm{~h}$, (Fig. $4.2 \mathrm{~d}$ ) resulted In the elimination of the peak at $15.8 \mathrm{~A}$ (this peak is characteristic of ' $\mathrm{B}$ ' type starches), an increase in intensity of the peak at $5.2 \mathrm{~A}_{4}$ and in the appearance of two new peaks centered at 5.9 and $3.8 \AA$. The $X$-ray pattern (Fig. $4.2 \mathrm{~d}$ ) after heat-moisture treatment (at $110^{\circ} \mathrm{C}$ ) resembled those of cereal starches (Hoover \& Vasanthan, 1994a).

The decrease in X-ray intensity on heat-moisture treatment (Table 4.2) can be attributed to crystallite disruption and/or to reorientation of the double helices forming the crystalline array. Imberty ot al. (1988) and Imberty \& Perez (1988) have shown that double helices of ' $A$ ' and ' $B$ ' type starches are packed in a pseudohexagonal array. The lattices of ' $B$ ' starch have a large void in which 36 water molecules can be accommodated. This void is not present in 'A' starch. In both ' $A$ ' and ' $\mathrm{B}$ ' arrangements there is a pairing of double helices that corresponds to $1.1 \mathrm{~nm}$ distance between the axes of the two double helices. A transformation from ' $B$ ' to 'A' starch occurs by rearrangement of a pair of double 
helices (Imberty et al., 1988). The extent of formation of 'A' type unit cells would therefore depend on the kinetic energy of the double helices involved in this arrangement. This seems plausible, since more ' $A$ ' type unit cells are formed When the temperature of heat-moisture treatment is increased from 100 to $110^{\circ} \mathrm{C}$ (Figs. 4.2c, d). Le Bail et al. (1993) also demonstrated the development of ' $A+B$ ' pattern from ' $B$ ' type short chain amylose (35\% moisture) when heated to $112^{\circ} \mathrm{C}$, whereas perfect ' $A$ ' type was obtained at $152^{\circ} \mathrm{C}$.

The increase in $\mathrm{X}$-ray intensities on defatting (Table 4.2) suggests that a clustering of the outer ' $A$ ' chains of amylopectin may have occurred resulting in the formation of new crystallites (that are perfectly arrayed to diffract $X$-rays). It is highly unlikely that the increase in X-ray intensity is due to reorientation of existing crystallites since the moisture and thermal energy $\left(82^{\circ} \mathrm{C}\right)$ during defatting is too low to impart the required kinetic energy for reorientation.

\subsubsection{Swelling factor (SF) and Amylose leaching (AML)}

The SF and AML at different temperatures $\left(50-90^{\circ} \mathrm{C}\right)$ are presented in Tables 4.3 and 4.4. The SF and AML of native, defatted and heat-moisture treated starches increased with rise in temperature (native > defatted > heatmoisture treated). Both defatting and heat-moisture treatment decreased SF and AML (Tables $4.3 \& 4.4$ ), which is in agreement with the resulted by Stute (1992), Hoover \& Vasanthan (1994a) and Hoover \& Manuel (1996a) . 


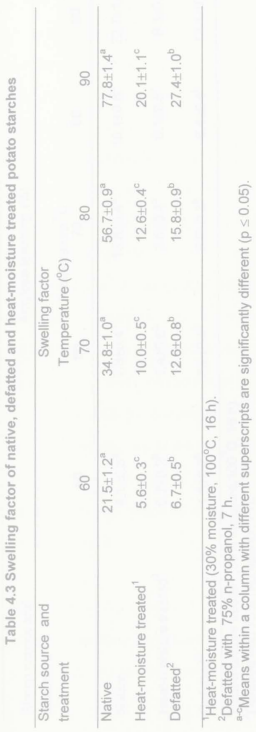




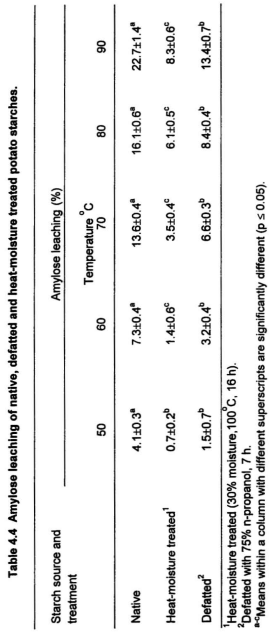


The decrease in AML, suggests interaction between amylose-amylose (AM-AM) and/or amylose-amylopectin (AM-AMP) chains, These interactions decrease the number of hydroxyl groups that can potentially bind to water molecules. This would then partially explain the decrease in SF on defatting and heat-moisture treatment. Tester \& Morrison (1990a,b) have shown, by comparative studies on non waxy and waxy maize starches, that swelling is primarily a property of amylopectin and that amylose is a diluent. Furthermore, Cooke \& Gidley (1992) have shown that the forces holding the granule together are mainly at the double helical level. The large decrease in SF on heat-moisture treatment implies that some of the double helices (free and/or present in crystalline lattices) may have unraveled on heat-moisture treatment. This seems plausible, since $\mathrm{X}$-ray diffraction intensities decrease on heat-moisture treatment (Fig. 4.2). Thus, both starch chain interactions and loss of double helical order contribute to the decrease in SF on heat-moisture treatment. The decrease in SF is less pronounced on defatting due to the interplay of two factors : 1) AM-AM and/or AM-AMP interactions which tend to decrease the SF and 2) the increase in crystallinity (Fig. 4.2) (which suggests an increase in double helical order) which tends to increase the SF.

\subsubsection{Differential Scanning Calorimetry}

The influence of defatting and heat-moisture treatment on gelatinization temperatures [ onset $\left(T_{0}\right)$, mid point $\left(T_{p}\right)$ and conclusion $\left(T_{c}\right)$ ] and gelatinization 
enthalpy $(\Delta H)$ are presented in Table 4.5. $T_{0}, T_{p}$ and $T_{c}$ increased on defatting and heat-moisture treatment (heat-moisture treatment > defatting). Gelatinization enthalpy decreased by $5.3 \mathrm{~J} / \mathrm{g}$ on heat-moisture treatment, and slightly increased on defatting. The gelatinization temperature range $\left(T_{\sigma}-T_{0}\right)$ increased by 2.5 and $6.0^{\circ} \mathrm{C}$, respectively. on defatting and heat-moisture treatment. Similar observations have also been reported on heat-moisture treatment and defatting of cereal, legume and tuber starches (Lorenz \& Kulp 1982; Donovan et al., 1983; Stute, 1992; Hoover \& Vasanthan, 1994a, Hoover \& Manuel, 1996a,b).

The gelatinization of starch is considered to be a solvent and heat induced melting of crystallites. When starch is heated in excess water, the water penetrates into the more accessible amorphous region of the starch granule, resulting in hydration and limited swelling. The swelling of the amorphous region imparts a stress on the crystalline region and thereby disrupts the polymer chains in the starch crystallites (Donovan, 1979). Therefore, any starch attributes that suppressed swelling would delay gelatinization and thus lead to a high $T_{0}, T_{p}$ and $T_{0}$. Cooke \& Gidley (1992) have shown that both crystalline and double helical order are lost concomitantly during gelatinization. Hoover \& Manuel (1996a) have shown that the increase in $T_{0}, T_{p}$ and $T_{c}$ on heat-moisture treatment of maize starches follows the order : amylomaize V (65.5\% amylose) $>$ normal maize $(29.9 \%$ amylose $)>$ waxy maize $(1.2 \%$ amylose). Furthermore, $T_{\sigma}-T_{0}$ remains unchanged in waxy maize, but increases respectively, by 6 and 
Table 4.5 Gelatinization parameters of native, defatted and heat-moisture treated starches

\begin{tabular}{|c|c|c|c|c|c|}
\hline \multirow{2}{*}{$\begin{array}{l}\text { Starch source and } \\
\text { treatment }\end{array}$} & \multicolumn{4}{|c|}{ Transition temperatures $\left({ }^{\circ} \mathrm{C}\right.$ ) } & \multirow{2}{*}{$\begin{array}{l}\text { Enthalpy } \\
\Delta H(J / g)^{3}\end{array}$} \\
\hline & $T_{0}{ }^{1}$ & $T_{p}{ }^{\prime}$ & $T_{c}{ }^{1}$ & $T_{c}-T_{0}^{2}$ & \\
\hline Native & $54.0 \pm 0.9^{\circ}$ & $61.0 \pm 0.6^{C}$ & $65.5 \pm 0.8^{c}$ & $11.5 \pm 0.3^{c}$ & $16.2 \pm 0.4^{8}$ \\
\hline Heat-moisture treated $^{5}$ & $63.5 \pm 0.4^{a}$ & $73.5 \pm 0.5^{3}$ & $81.0 \pm 0.3^{a}$ & $17.5 \pm 0.1^{\mathrm{B}}$ & $10.9 \pm 0.8^{\circ}$ \\
\hline Defatted ${ }^{6}$ & $59.0 \pm 0.5^{b}$ & $65.5 \pm 0.4^{b}$ & $73.0 \pm 0.3^{b}$ & $14.0 \pm 0.2^{b}$ & $17.1 \pm 0.2^{a}$ \\
\hline
\end{tabular}

${ }^{1} T_{0}, T_{p}, \& T_{c}$ indicate respectively, the temperature of onset, mid point, and end of gelatinization.

${ }^{2}$ Gelatinization temperature range.

${ }^{3}$ Enthalpy of gelatinization.

${ }^{4}$ Parent starch treated with $\mathrm{NaOH}$ and $\mathrm{Na}_{2} \mathrm{SO}_{4}$ at $40^{\circ} \mathrm{C}$, but without addition of propylene oxide.

${ }^{5}$ Heat-moisture treated $\left(30 \%\right.$ moisture, $\left.100^{\circ} \mathrm{C}, 16 \mathrm{~h}\right)$.

${ }^{6}$ Defatted with $75 \%$ n-propanol, $7 \mathrm{~h}$.

${ }^{\mathrm{a}-\mathrm{c}}$ Means within a column with different superscripts are significantly different ( $\left.p \leq 0.05\right)$. 
$8^{\circ} \mathrm{C}$. on heat-moisture treatment of normal maize and amylomaize $V$ starches. This indicates that the increase in $T_{0}, T_{p}$ and $T_{c}$ (Table 4.5) reflect melting of crystallites that were formed solely due to interaction between AM-AM and AMAMP chains during heat-moisture treatment. As discussed earlier, interaction between AM-AM and/or AM-AMP chains are stronger on heat-moisture treatment. Therefore, suppression of granule swelling would be greater on heatmoisture treatment than on defatting (Table 4.3). Consequently, the destabilization effect of the amorphous regions on crystallite melting would be less pronounced in heat-moisture treated than in defatted granules. This would then explain the higher increases in $T_{0}, T_{p}$ and $T_{c}$ on heat-moisture treatment (Table 4.5). The increase in $T_{c}-T_{0}$ on defatting and heat-moisture treatment reflects the melting of crystallites (that were formed by interaction between AMAM and/or AM-AMP chains) of different stability. The decrease in $\Delta \mathrm{H}$ on heatmoisture treatment suggests that double helices present in crystalline and noncrystalline arrays may have disrupted under the conditions prevailing during heat-moisture treatment. The increase in $\Delta \mathrm{H}$ on defatting (Table 4.5) suggests that additional double helices may have formed under the conditions prevailing during defatting.

\subsubsection{Brabender viscosities (Pasting characteristics)}

The pasting characteristics of the starches at a concentration of $6 \%$ (w/v) and $\mathrm{pH} 5.5$ were investigated with the Brabender viscoamylograph and the 
results are presented in Table 4.6. Heat-moisture treatment and defatting increased the pasting temperature, by 27.7 and $24.7^{\circ} \mathrm{C}$, respectively. The viscosity at $95^{\circ} \mathrm{C}$ decreased by $1140 \mathrm{BU}$ (Brabender Units) and $560 \mathrm{BU}$, respectively, on heat-moisture treatment and defatting. The viscosity during the holding cycle (at $95^{\circ} \mathrm{C}$ ) decreased by $670 \mathrm{BU}$ in native potato starch, whereas it increased by $170 \mathrm{BU}$ and $440 \mathrm{BU}$, respectively, in heat-moisture treated and defatted potato starches. All three starches showed an increase in viscosity during the cooling cycle (defatted > heat-moisture treated > native) [Table 4.6]. Similar observation have been reported for heat-moisture treated and defatted legume (Hoover et al., 1993; Hoover \& Vasanthan, 1994a) and tuber (Stute. 1992; Hoover \& Vasanthan, 1994a) starches.

The increased pasting temperature and the decreased viscosity at $95^{\circ} \mathrm{C}$ on heat-moisture treatment (Table 4.6) reflects, to a large extent, the decrease in granular crystallinity (which decrease both granular rigidity and the volume fraction occupied by the swollen granules) and the interaction between AM-AM and/or AM-AMP chains (which decreases granular swelling). The pasting curve of defatted potato starch reflects the interplay between the increase in granule crystallinity (which increases both granular rigidity and the volume fraction occupied by the swollen granules) and the interaction between starch chains (which decreases granular swelling). This would then explain the more pronounced changes in the pasting properties on heat-moisture treatment. In native potato starch, the breakdown in viscosity during the holding (Table 4.6) 
Table 4.6 Brabender viscosities (Pasting properties) of native, defatted and heat-moisture treated potato starches

\begin{tabular}{|c|c|c|c|c|}
\hline Starch source and treatment & $\begin{array}{c}\text { Pasting } \\
\text { temperature }\left({ }^{\circ} \mathrm{C}\right)\end{array}$ & $\begin{array}{c}\text { Viscosity at } 95^{\circ} \mathrm{C} \\
\left(\mathrm{BU}^{\dagger}\right)\end{array}$ & $\begin{array}{c}\text { Viscosity after } 30 \\
\text { min at } 95^{\circ} \mathrm{C} \\
\left(\mathrm{BU}^{1}\right)\end{array}$ & $\begin{array}{c}\text { Viscosity at } 50^{\circ} \mathrm{C} \\
\left(\mathrm{BU}^{1}\right)\end{array}$ \\
\hline Native & $62.3 \pm 0.5^{c}$ & $1190 \pm 10^{a}$ & $520 \pm 10^{b}$ & $715 \pm 10^{6}$ \\
\hline Heat-moisture treated ${ }^{2}$ & $90.0 \pm 0.5^{\mathrm{a}}$ & $50 \pm 5^{\circ}$ & $220 \pm 5^{\circ}$ & $350 \pm 5^{c}$ \\
\hline Defatted $^{3}$ & $87.0 \pm 1.0^{b}$ & $630 \pm 5^{b}$ & $1070+15^{a}$ & $1500 \pm 15^{\mathrm{a}}$ \\
\hline $\begin{array}{l}\text { TBabender units. } \\
{ }^{2} \text { Heat-moisture treated ( } 30 \% \text { r } \\
{ }^{3} \text { Defatted with } 75 \% \text { n-propano } \\
{ }^{3-c} \text { Means within a column with }\end{array}$ & ure, $100^{\circ} \mathrm{C}, 16 \mathrm{~h}$ ). & & (U.0) & \\
\hline
\end{tabular}


cycle (at $95^{\circ} \mathrm{C}$ ) can be attributed to weak associative bonding forces within the granule interior. However, the additional interactions that occur between AM-AM and AM-AMP chains on defatting and heat-moisture treatment impart shear and thermal stability during the holding cycle.

\subsection{THE EFFECT OF ALKALINE TREATMENT ON THE STRUCTURE AND PROPERTIES OF NATIVE, DEFATTED AND HEAT-MOISTURE TREATED STARCHES}

Sodium hydroxide and sodium sulphate are used in the preparation of hydroxypropylated starches to ensure good reaction efficiency and to depress granular swelling and gelatinization. Therefore, it was deemed necessary to investigate the influence of above reagents (under the conditions prevaling during hydroxypropylation, but in the absence of propylene oxide) on the structure and properties of native, defatted and heat-moisture treated starches.

\subsubsection{Morphological granular characteristics}

The scanning electron micrographs showed that alkali treatment did not alter the shape (oval to elliptical) and appearence of native, defatted and heatmoisture treated starch granules. 


\subsubsection{X-ray diffraction of alkall treated starches}

The $\mathrm{X}$-ray intensities and $\mathrm{X}$-ray patterns of the control starches are presented respectively in Table 4.7 and Fig. 4.3. The X-ray intensities and X-ray patterns of native and defatted starches were drastically altered after alkaline treatment. The altered X-ray patterns (neither ' $A$ ', ' $B$ ' or ' $C$ ') were identical in both starches (Figs. 4.3 a,c). However, for heat-moisture treated starch, X-ray intensities decreased only marginally (Table 4.7) and the X-ray pattern remained unchanged after alkaline treatment (Fig. 4.3b).

4.3.3 Swelling factor (SF) and amylose leaching (AML) of alkali treated starches

In native, defatted and heat-moisture treated starches the SF at all temperatures $\left(60-90^{\circ} \mathrm{C}\right)$ increased after alkaline treatment (Table 4.8). The increase in SF followed the order : native > defatted > heat-moisture treated. However, AML (at $90^{\circ} \mathrm{C}$ ) in all three starches remained unaffected (Table 4.9) after alkaline treatment.

\subsubsection{Differential Scanning Calorimetry of alkali treated starches}

In all three starches (native, defatted and heat-moisture treated starches with no added propylene oxide), the gelatinization transition temperatures and the gelatinization transition temperature range $\left(T_{0}-T_{0}\right)$ remained unaltered after 
Table 4.7 X-ray diffraction intensities of the major peaks of native, defatted and heat-moisture treated potato starches before and after alkaline treatment

\begin{tabular}{|c|c|c|c|c|c|c|c|c|}
\hline \multirow{2}{*}{$\begin{array}{l}\text { Starch source and } \\
\text { treatment } \\
\text { Native }\end{array}$} & \multirow{2}{*}{$\begin{array}{l}\text { Moisture } \\
\text { content } \\
(\%)\end{array}$} & \multicolumn{7}{|c|}{ Interplanar spacing (d) in $\AA$ with intensities (cps) ${ }^{1}$} \\
\hline & & & & & & & & \\
\hline before treatment & 9.5 & $15.8(581)$ & $5.2(2362)$ & $4.5(327)$ & $3.9(867)$ & - & - & - \\
\hline after treatment ${ }^{2}$ & 9.3 & - & $5.3(1212)$ & $4.0(576)$ & - & - & - & \\
\hline \multicolumn{9}{|c|}{ Heat-moisture treated ${ }^{3}$} \\
\hline before treatment & 9.3 & $16.0(201)$ & $5.2(2020)$ & $4.0(850)$ & - & - & - & - \\
\hline after treatment $t^{2}$ & 9.4 & $15.8(369)$ & $5.2(1949)$ & $3.8(1069)$ & - & - & - & - \\
\hline \multicolumn{9}{|l|}{ Defatted $^{4}$} \\
\hline before treatment & 9.3 & $15.0(570)$ & $5.2(1979)$ & $4.6(525)$ & $3.9(1022)$ & $3.8(935)$ & $3.3(590)$ & $2.9(491)$ \\
\hline after treatment ${ }^{2}$ & 9.5 & - & $5.4(1426)$ & - & $3.9(698)$ & - & - & - \\
\hline
\end{tabular}

\footnotetext{
TCounts per second.

${ }_{2}^{2}$ Parent starch treated with $\mathrm{NaOH}$ and $\mathrm{Na}_{2} \mathrm{SO}_{4}$ at $40^{\circ} \mathrm{C}$.

${ }^{3}$ Heat-moisture treated $\left(30 \%\right.$ moisture, $\left.100^{\circ} \mathrm{C}, 16 \mathrm{~h}\right)$.

${ }^{4}$ Defatted with 75\% n-propanol, $7 \mathrm{~h}$.
} 
Fig. 4.3 X-ray diffraction patterns of native, heat-moisture treated (HMT) [30\% moisture, $100^{\circ} \mathrm{C}, 16 \mathrm{~h}$ ) and defatted $(75 \%$ n-propanol, $7 \mathrm{~h})$ potato starches after alkaline treatment ( $\mathrm{NaOH}$ and $\mathrm{Na}_{2} \mathrm{SO}_{4}$ ) at $40^{\circ} \mathrm{C}$. (A) native after alkaline treatment (MC\% 9.3), (B) HMT after alkaline treatment (MC 9.4\%), (C) defatted after alkaline treatment (MC 9.5\%). 


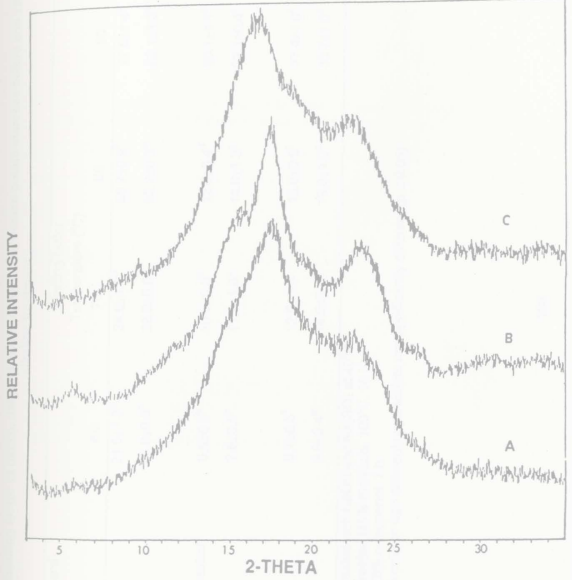




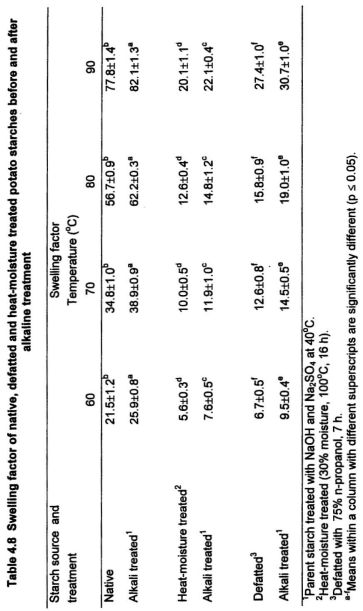

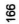


Table 4.9 Amylose leaching (at $90^{\circ} \mathrm{C}$ ) of native, defatted and heat-moisture treated potato starches before and after alkaline treatment

Starch source and treatment

Amylose leaching (\%)

Native

Alkali treated ${ }^{1}$

Heat-moisture treated ${ }^{2}$

Alkali treated ${ }^{1}$

Defatted $^{3}$

Alkali treated ${ }^{1}$
$22.7 \pm 1.4^{\mathrm{a}}$

$21.9 \pm 1.0^{\circ}$

$8.3 \pm 0.6^{\circ}$

$8.5 \pm 0.9^{\circ}$

$13.4 \pm 0.7^{\circ}$

$13.8 \pm 0.5^{b}$

\footnotetext{
TParent starch treated with $\mathrm{NaOH}$ and $\mathrm{Na}_{2} \mathrm{SO}_{4}$ at $40^{\circ} \mathrm{C}$.

${ }^{2}$ Heat-moisture treated $\left(30 \%\right.$ moisture, $\left.100^{\circ} \mathrm{C}, 16 \mathrm{~h}\right)$.

${ }^{3}$ Defatted with $75 \%$ n-propanol, $7 \mathrm{~h}$.

${ }^{-c}$ Means within a column with different superscripts are significantly different ( $p \leq$ 0.05).
} 
treatment. However, $\Delta \mathrm{H}$ decreased by $5.3,1.3$ and $1.2 \mathrm{~J} / \mathrm{g}$, respectively, in native, heat-moisture treated and defatted potato starches after alkali treatment (Table 4.10).

\subsubsection{Brabender viscosities (pasting characteristics) of alkali treated starches}

In all three (native, defatted and heat-moisture treated) starches, the pasting temperature changed only marginally on alkaline treatment (Table 4.11). However, the viscosity at $95^{\circ} \mathrm{C}$ decreased by 440,20 and $300 \mathrm{BU}$, respectively, in native, heat-moisture treated and defatted starches (Table 4.11). Alkaline treatment improved the thermal stability (during the holding period at $95^{\circ} \mathrm{C}$ ) of all three starches.

The principal sequence of events during gelatinization is postulated to be disordering of crystalline clusters, then dissociation of double helices to give loosely ordered (semi random) chains (Tester \& Morrison, 1990a). The gelatinization endotherm is given by clusters of double helices (formed from the comparatively short free ends of A and B chains in amylopectin) rather than by separated double helices (Tester \& Morrison, 1990a). The $\Delta H$ values mainly reflect the loss of double helical order rather than loss of crystalline register (Cook \& Gidley, 1992; Whittam et al., 1990, 1991). This means that the forces 
Table 4.10 Gelatinization parameters of native, defatted and heat-moisture treated starches before and after alkaline treatment

\begin{tabular}{|c|c|c|c|c|c|}
\hline \multirow{2}{*}{$\begin{array}{l}\text { Starch source and } \\
\text { treatment }\end{array}$} & \multicolumn{4}{|c|}{ Transition temperatures $\left({ }^{\circ} \mathrm{C}\right)$} & \multirow{2}{*}{$\begin{array}{l}\text { Enthalpy } \\
\qquad \Delta \mathrm{H}(\mathrm{J} / \mathrm{g})^{3}\end{array}$} \\
\hline & $T_{0}{ }^{1}$ & $T_{p}{ }^{1}$ & $T_{c}^{\prime}$ & $\mathrm{T}_{\mathrm{c}-\mathrm{T}_{0}{ }^{2}}$ & \\
\hline Native & $54.0 \pm 0.9^{8}$ & $61.0 \pm 0.6^{a}$ & $65.5 \pm 0.8^{a}$ & $11.5 \pm 0.3^{a}$ & $16.2 \pm 0.4^{8}$ \\
\hline Alkali treated ${ }^{4}$ & $54.5 \pm 0.2^{\mathrm{n}}$ & $61.4 \pm 0.1^{\circ}$ & $66.4 \pm 0.6^{\mathrm{a}}$ & $11.9 \pm 0.2^{\mathrm{a}}$ & $10.9 \pm 0.2^{b}$ \\
\hline Heat-moisture treated ${ }^{5}$ & $63.5 \pm 0.4^{\mathrm{c}}$ & $73.5 \pm 0.5^{c}$ & $81.0 \pm 0.3^{c}$ & $17.5 \pm 0.1^{\mathrm{c}}$ & $10.9 \pm 0.8^{c}$ \\
\hline Alkali treated ${ }^{4}$ & $63.0 \pm 0.0^{\mathrm{c}}$ & $73.0 \pm 0.2^{c}$ & $80.0 \pm 0.1^{d}$ & $17.0 \pm 0.4^{\mathrm{c}}$ & $9.6 \pm 0.1^{d}$ \\
\hline Defatted $^{\theta}$ & $59.0 \pm 0.5^{\circ}$ & $65.5 \pm 0.4^{\circ}$ & $73.0 \pm 0.3^{\circ}$ & $14.0 \pm 0.2^{\mathrm{e}}$ & $17.1 \pm 0.2^{\circ}$ \\
\hline Alkali treated ${ }^{4}$ & $58.0 \pm 0.4^{\circ}$ & $65.0 \pm 0.1^{\circ}$ & $72.5 \pm 0.3^{\circ}$ & $14.5 \pm 0.2^{\circ}$ & $15.9 \pm 0.3^{\prime}$ \\
\hline
\end{tabular}

${ }^{1} T_{0}, T_{p} \& T_{0}$ indicate respectively, the temperature of onset, mid point, and end of gelatinization.

${ }^{2}$ Gelatinization temperature range.

${ }^{3}$ Enthalpy of gelatinization.

${ }^{4} \mathrm{Parent}$ starch treated with $\mathrm{NaOH}$ and $\mathrm{Na}_{2} \mathrm{SO}_{4}$ at $40^{\circ} \mathrm{C}$, but without addition of propylene oxide.

${ }^{5} \mathrm{Heat}-$ moisture treated $\left(30 \%\right.$ moisture, $\left.100^{\circ} \mathrm{C}, 16 \mathrm{~h}\right)$.

${ }^{6}$ Defatted with $75 \%$ n-propanol, $7 \mathrm{~h}$.

${ }^{0-0}$ Means within a column with different superscripts are significantly different ( $p \leq 0.05$ ). 
Table 4.11 Brabender viscosities (Pasting properties) of native, defatted and heat-moisture treated potato starches before and after alkaline treatment

\begin{tabular}{|c|c|c|c|c|}
\hline Starch source \& treatment & $\begin{array}{c}\text { Pasting } \\
\text { temperature }\left({ }^{\circ} \mathrm{C}\right)\end{array}$ & $\begin{array}{c}\text { Viscosity at } 95^{\circ} \mathrm{C} \\
\left(\mathrm{BU}^{1}\right)\end{array}$ & $\begin{array}{c}\text { Viscosity after } 30 \\
\text { min at } 95^{\circ} \mathrm{C} \\
\left(\mathrm{BU}^{2}\right)\end{array}$ & $\begin{array}{c}\text { Viscosity at } 50^{\circ} \mathrm{C} \\
\left(\mathrm{BU}^{1}\right)\end{array}$ \\
\hline Native & $62.3 \pm 0.5^{b}$ & $1190 \pm 10^{2}$ & $520 \pm 10^{b}$ & $715 \pm 10^{b}$ \\
\hline Alkali treated ${ }^{2}$ & $64.5 \pm 0.5^{a}$ & $750 \pm 10^{b}$ & $870 \pm 10^{a}$ & $1140 \pm 20^{\mathrm{a}}$ \\
\hline Heat-moisture treated ${ }^{3}$ & $90.0 \pm 0.5^{d}$ & $50 \pm 5^{c}$ & $220 \pm 5^{d}$ & $350 \pm 5^{\circ}$ \\
\hline Alkali treated ${ }^{2}$ & $91.5 \pm 0.5^{c}$ & $30 \pm 5^{d}$ & $130 \pm 10^{\circ}$ & $230+5^{d}$ \\
\hline Defatted ${ }^{4}$ & $87.0 \pm 1.0^{\circ}$ & $630 \pm 5^{e}$ & $1070 \pm 15^{\circ}$ & $1500 \pm 15^{e}$ \\
\hline Alkali treated ${ }^{2}$ & $87.5 \pm 1.0^{9}$ & $330 \pm 5^{1}$ & $940+5^{\prime}$ & $1460 \pm 10^{f}$ \\
\hline
\end{tabular}

\footnotetext{
'Brabender units.

${ }^{2}$ Parent starch treated with $\mathrm{NaOH}$ and $\mathrm{Na}_{2} \mathrm{SO}_{4}$ at $40^{\circ} \mathrm{C}$.

${ }^{3}$ Heat-moisture treated $\left(30 \%\right.$ moisture, $\left.100^{\circ} \mathrm{C}, 16 \mathrm{~h}\right)$.

${ }^{4}$ Defatted with $75 \%$ n-propanol, $7 \mathrm{~h}$.

${ }^{a-}$ Means within a column with different superscripts are significantly different $(p \leq 0.05)$.
} 
responsible for structural stability of starch granules are largely at the double helical level, and that chain packing energy contributions are insignificant.

The lack of influence of the alkaline treatment on $T_{0,} T_{p}$ and $T_{c}$, (Table 4.10) suggests that double helices comprising the crystalline clusters are not disrupted during alkaline treatment. Therefore, the extent of decrease in $\Delta \mathrm{H}$, suggests that the alkaline treatment disrupts double helices (native > defatted > heat-moisture treated) present within the amorphous regions of the granule. The smaller decrease in $\Delta \mathrm{H}$ for alkali treated defatted and heat-moisture treated starches, suggest that less double helices are available within the amorphous regions of these starches for disruption by alkali. This indicates, that during the process of defalting and heat-moisture treatment the thermal energy imparted to the starch chains (heat-moisture treatment > defatting) may have caused some disruption of double helices within the amorphous domains of the granule. Double helices disrupt to a greater extent on heat-moisture treatment than on defatting due to the higher thermal energy imparts to the starch chains during heat-moisture treatment $\left(100^{\circ} \mathrm{C}\right.$ vs $82^{\circ} \mathrm{C}$ during defatting).

The extent of increase in SF (Table 4.8) on alkaline treatment (native > defatted > heat-moisture treated) also suggests that double helices within amorphous regions disrupt (exposes more hydroxyl groups for water interaction) on alkaline treatment.

The decrease in X-ray intensities and the change in the X-ray patterns (Fig 4.3) of alkali treated native and defatted starches are more likely due to a 
change in crystallite orientation rather than crystallite disruption (crystallite disruption would have altered the gelatinization transition temperatures).

It is necessary at this stage to give a brief description of the 'A' and 'B' type $X$-ray patterns of starches, which will allow a subsequent discussion of the changes in $\mathrm{X}$-ray patterns on alkaline treatment. The ' $\mathrm{A}$ ' $\mathrm{X}$-ray pattern which is typical of cereal starches, consists of chains which are crystallized in a monoclinic lattice unit having the maltotriose as a repeating unit and 4 water molecules per unit cell. The hexagonal sub cell of the ' $B$ ' pattern of tuber starches has a more 'open' packing of double helices, and a maltose moiety as an asymmetric unit, and 36 water molecules (at $\sim 27 \%$ w/w hydration) per unit cell. Fifty percent of the water is tightly bound to the chains and the other half is only connected to the other water (Imberty \& Perez, 1988; Imberty et al., 1988). This indicates that the number of water molecules within the unit cell of the starches used in this study follow the order : native ('B' type unit cell) $>$ defatted ('A'+'B' type unit cell) > heat-moisture treated (mainly 'A' type unit cells). Crystallite reorientation on alkaline treatment (native $>$ defatted) can be attributed to a decrease (native > defatted) in the number of water molecules (due to the dehydrating action of $\mathrm{Na}_{2} \mathrm{SO}_{4}$ ) within the unit cells of native and defatted starches. Crystallite reorientation does not occur to any significant extent in heat-moisture treated potato starch, since the sulphate ions are unable to access the water molecules within the compactly packed 'A' type unit cells. 
The decrease in viscosity $95^{\circ} \mathrm{C}$ (native > defatted > heat-moisture treated), and the increase in granular resistance towards shear during the holding cycle at $95^{\circ} \mathrm{C}$ (native > defatted > heat-moisture treated) on alkaline treatment (Table 4.11) suggest an increase in granular rigidity. Amylose leaching (Table 4.9) and DSC (Table 4.10) studies have shown that interactions do not occur between starch chains on alkaline treatment. Therefore, the increase in granular rigidity may have been solely due to crystallite reorientation.

\subsection{EFFECT OF DEFATTING AND HEAT-MOISTURE TREATMENT ON THE HYDROXYPROPYLATION OF NATIVE POTATO STARCH}

The extent of hydroxypropylation was quantified and expressed in terms of Molar substitution (MS). The MS of native, defatted and heat-moisture treated potato starches by hydroxypropyl groups at different levels of propylene oxide (2$25 \% \mathrm{v} / \mathrm{w})$ are presented in Fig. 4.4. There were no significant difference $(p>$ 0.05 ) in MS between the starches up to a level of $5 \%$ propylene oxide. Thereafter, the extent of MS followed the order : heat-moisture treated > native > defatted.

The extent of MS (at 10\% propylene oxide) increased with the time course of heat-moisture treatment, but decreased during the time course of defatting (Fig. 4.5). As described earlier, the amount of amorphous regions within the starch granule increases on heat-moisture treatment, but decrease on defatting. Thus, the differences in MS among the three starches at the same level of 
Fig. 4.4 The level of molar substitution (MS) of native, heat-moisture treated and defatted potato starch by hydroxypropyl groups. 


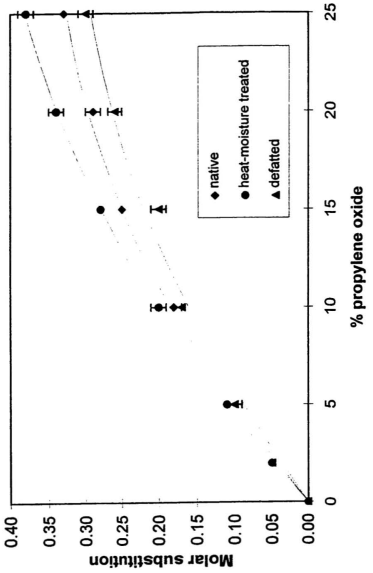


Fig. 4.5 Influence of time of defatting ( $75 \%$ n-propanol) and heat-moisture treatment $\left(30 \%\right.$ moisture, $\left.100^{\circ} \mathrm{C}\right)$ on MS level. 


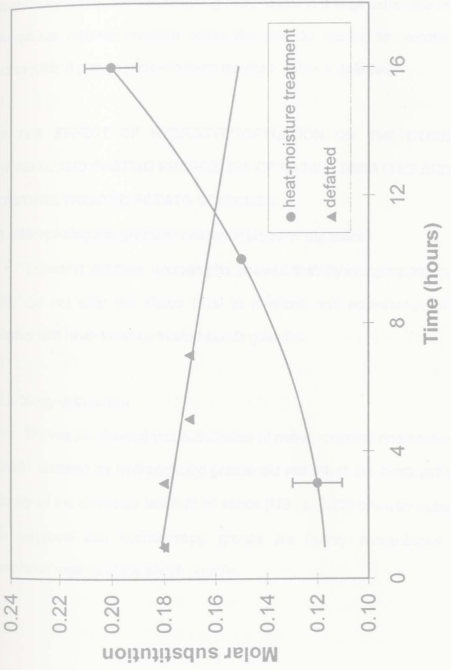


propylene oxide and the changes in the level of MS with increase in time of heatmoisture treatment and defatting (Fig. 4.5), reflect to a large extent the amount of amorphous regions available within the granule interior for substitution by hydroxypropyl groups (heat-moisture treated > native > defatted).

\subsection{THE EFFECT OF HYDROXYPROPYLATION ON THE STRUCTURE, THERMAL AND PASTING PROPERTIES OF NATIVE, DEFATTED AND HEAT- MOISTURE TREATED POTATO STARCHES}

\subsubsection{Morphological granular characteristics of the starch}

Scanning electron micrographs showed that hydroxypropylation (MS $\leq$ 0.28) did not alter the shape (oval to elliptical) and appearence of native, defatted and heat-moisture treated starch granules.

\subsubsection{X-ray diffraction}

The results showed that substitution of native, defatted and heat-moisture treated starches by hydroxypropyl groups did not affect the X-ray pattern and intensity of the diffracted beam at all levels (MS $\leq 0.28$ ) of molar substitution. This suggests that hydroxypropyl groups are mainly concentrated in the amorphous regions of the starch granule. 


\subsubsection{Swelling factor (SF)}

The SF of control and hydroxypropylated potato starches in the temperature range $60-90^{\circ} \mathrm{C}$ are presented in Table 4.12. At $60,70,80$ and $90^{\circ} \mathrm{C}$. the SF of hydroxypropylated native starch increased (with reference to control starch), by $28.6,15.4,14.3$ and $9.6 \%$, respectively as the MS increased from 0 to 0.05 (Table 4.12). Thereafter, the SF at $60^{\circ} \mathrm{C}$ increased only marginally with increase in MS $(0.05 \rightarrow 0.25)$. However, at 70 and $80^{\circ} \mathrm{C}$, the SF began to decrease at MS 0.25. However, at $90^{\circ} \mathrm{C}$ the decline in SF was seen at MS 0.11. In hydroxypropylated heat-moisture treated starches, the SF at 60, 70, 80 and $90^{\circ} \mathrm{C}$ increased respectively, by $5.3,18.5,40.5$ and $45 \%$ as the MS increased from 0 to 0.05 . Thereafter, the SF at 60,70 and $80^{\circ} \mathrm{C}$ increased only marginally with increase in MS $(0.05 \rightarrow 0.28)$ (Table 4.12). However, at $90^{\circ} \mathrm{C}$, the SF began to decrease at MS 0.28. In hydroxypropylated defatted starches, the increase in SF $\left[60^{\circ} \mathrm{C}(6.2 \%), 70^{\circ} \mathrm{C}(23.0 \%), 80^{\circ} \mathrm{C}(63.0 \%)\right.$ and $\left.90^{\circ} \mathrm{C}(66.4 \%)\right]$ with increase in MS $(0 \rightarrow 0.05)$, was much higher than that observed for native and heatmoisture treated starches at the same level of MS (0.05). Furthermore, in defatted starches the SF continued to increase with increase in MS $(0.05 \rightarrow$ 0.20) at all temperatures (Table 4.12).

The introduction of bulky hydroxypropyl groups into the starch molecule may be considered as a reaction which disrupts associative hydrogen bonds Within the amorphous regions of the starch granule. Thus, the increase in SF 
Table 4.12 Swelling factor of hydroxypropyl native, defatted and heat-moisture treated potato starches

\begin{tabular}{|c|c|c|c|c|c|}
\hline \multirow[t]{2}{*}{$\begin{array}{l}\text { Starch source \& } \\
\text { treatment }\end{array}$} & \multirow[t]{2}{*}{$\begin{array}{c}\text { Molar } \\
\text { substitution }\end{array}$} & \multicolumn{4}{|c|}{$\begin{array}{l}\text { Swelling factor } \\
\text { Temperature }\left({ }^{\circ} \mathrm{C}\right)\end{array}$} \\
\hline & & 60 & 70 & 80 & 90 \\
\hline Native & $\begin{array}{l}0.00^{1} \\
0.05 \\
0.11 \\
0.18 \\
0.25\end{array}$ & $\begin{array}{l}25.9 \pm 0.8^{\mathrm{d}} \\
33.3 \pm 0.8^{\mathrm{c}} \\
36.0 \pm 0.6^{\mathrm{b}} \\
37.6 \pm 0.6^{\mathrm{a}} \\
38.0 \pm 0.4^{\mathrm{a}}\end{array}$ & $\begin{array}{l}38.9 \pm 0.9^{c} \\
44.9 \pm 0.6^{b} \\
49.8 \pm 0.6^{a} \\
50.2 \pm 1.3^{a} \\
39.6 \pm 1.4^{c}\end{array}$ & $\begin{array}{l}62.2 \pm 0.3^{\mathrm{b}} \\
71.1 \pm 0.8^{\mathrm{a}} \\
71.1 \pm 0.2^{\mathrm{a}} \\
71.9 \pm 0.4^{\mathrm{a}} \\
58.5 \pm 1.0^{\mathrm{c}}\end{array}$ & $\begin{array}{l}82.1 \pm 1.3^{b} \\
90.0 \pm 0.9^{a} \\
82.6 \pm 0.4^{b} \\
81.5 \pm 0.8^{b} \\
71.9 \pm 0.6^{d}\end{array}$ \\
\hline Heat-moisture treated ${ }^{2}$ & $\begin{array}{l}0.00^{1} \\
0.05 \\
0.11 \\
0.20 \\
0.28\end{array}$ & $\begin{array}{c}7.6 \pm 0.5^{h} \\
8.0 \pm 1.2^{h} \\
11.1 \pm 0.8^{\circ} \\
12.6 \pm 0.4^{1} \\
19.5 \pm 0.6^{\circ}\end{array}$ & $\begin{array}{l}11.9 \pm 1.0^{h} \\
14.1 \pm 1.3^{h} \\
17.2 \pm 0.3^{0} \\
18.7 \pm 0.7^{\prime} \\
21.9 \pm 0.7^{\circ}\end{array}$ & $\begin{array}{l}14.8 \pm 1.2^{h} \\
20.8 \pm 0.6^{\natural} \\
22.5 \pm 0.7^{\natural} \\
24.6 \pm 0.5^{\circ} \\
25.7 \pm 0.7^{\circ}\end{array}$ & $\begin{array}{l}22.1 \pm 0.4^{\theta} \\
32.1 \pm 0.4^{\prime} \\
33.0 \pm 1.1^{\prime} \\
35.2 \pm 1.0^{\circ} \\
32.0 \pm 1.2^{\prime}\end{array}$ \\
\hline Defatted $^{3}$ & $\begin{array}{l}0.00^{1} \\
0.05 \\
0.10 \\
0.17 \\
0.20\end{array}$ & $\begin{array}{l}9.5 \pm 0.4^{m} \\
15.4 \pm 0.4^{\prime} \\
16.6 \pm 0.2^{k} \\
20.4 \pm 0.5^{\prime} \\
28.0 \pm 0.6^{\prime}\end{array}$ & $\begin{array}{l}14.5 \pm 0.5^{m} \\
17.9 \pm 0.3^{1} \\
20.5 \pm 0.4^{k} \\
26.9 \pm 1.0^{\prime} \\
38.0 \pm 1.1^{\prime}\end{array}$ & $\begin{array}{l}19.0 \pm 1.0^{m} \\
31.1 \pm 0.4^{\prime} \\
38.1 \pm 0.8^{k} \\
43.1 \pm 0.5^{\prime} \\
55.8 \pm 1.0^{\prime}\end{array}$ & $\begin{array}{c}30.7 \pm 1.0^{m} \\
51.1 \pm 0.8^{\prime} \\
56.4 \pm 0.7^{k} \\
60.2 \pm 0.6^{\prime} \\
70.6 \pm 0.6^{\prime}\end{array}$ \\
\hline
\end{tabular}


with increase in MS could be attributed to interaction of water molecules with the released hydroxyl groups of the starch chains (which were previously hydrogen bonded), and with the hydroxyl group of the hydroxypropyl chain. Polysubstitution on hydroxypropyl substituents already present will not increase the amount of hydroxyl groups. Thus, the marginal increase in SF with increase in MS could only be attributed to increased disruption of the associative hydrogen bonds by the hydroxypropyl groups. The decrease in SF observed at 70 and $80^{\circ} \mathrm{C}$ for native hydroxypropylated potato starch at MS 0.25 could be attributed to disruption (due to increased flexibility of the hydroxypropyl chains at these temperatures) of the associative bonding forces within the amorphous regions of the granule. This disruption of local order would reduce the rigidity of the granule, thereby decreasing its ability to swell. This seems plausible since at $90^{\circ} \mathrm{C}$ the decrease in SF begins at a much lower MS $(0.11)$.

As discussed earlier, heat-moisture treatment causes additional interactions to occur between starch chains in the amorphous region. This, would then explain why the observed decrease in SF only occurs at higher temperatures $\left(90^{\circ} \mathrm{C}\right)$ and at higher levels of MS $(0.28)$ in hydroxypropylated heatmoisture treated starch.

The increase in granular crystallinity that occurs on defatting (Fig. 4.2), increases granular rigidity. Thus, in defatted starch, even though associative bonding forces (within the amorphous regions) are disrupted (by heat and by the motion of the flexible hydroxypropyl groups), the granules do not collapse to the 
same extent as in native and heat-moisture treated starches. The increase in SF with increase in MS (for defatted starch) within the temperature range of $60-90^{\circ} \mathrm{C}$ thus stands explained (Table 4.12).

\subsubsection{Amylose leaching (AML)}

The extent of amylose leaching at $90^{\circ} \mathrm{C}$ decreased with increase in MS (Table 4.13). The extent of AML at this temperature was 4.9, 2.1 and 4.6, respectively for native (MS 0.25), heat-moisture treated (MS 0.28) and defatted (MS 0.20) starches. The interaction of the bulky hydroxypropyl groups with amylose chains probably prevents their diffusion out of the granule during thermal treatment.

\subsubsection{Differential scanning calorimetry (DSC)}

The enthalpies $(\Delta \mathrm{H})$ of gelatinization and the gelatinization transition temperatures $\left(T_{0}, T_{p}\right.$ and $\left.T_{c}\right)$ of native, defatted and heat-moisture treated hydroxypropylated starches are presented in Table 4.14. In all starches, increase in the level of MS resulted in a decrease in $\Delta H, T_{0}, T_{p}$ and $T_{c}$ and a broadening of the gelatinization temperature range $\left(T_{c}-T_{0}\right)$. DSC results, similar to this study, have been reported for hydroxypropylated pea starch (Hoover et al., 1988), hydroxypropylated rice starch (Seow \& Thevamalar, 1993), hydroxypropylated potato starch (Kim \& Eliasson, 1993) and hydroxypropylated maize starches 
Table 4.13 Amylose leaching of hydroxypropyl native, defatted and heatmoisture treated starches

siarch source and

Molar substitution

Amylose leaching (\%)

veatment

Native

$0.00^{1}$

0.05

0.11

0.18

0.25

$4.9 \div 0.2^{6}$

Heat-moisture treated ${ }^{2}$

$0.00^{7}$

$8.5 \pm 0.9^{f}$

0.05

$7.6 \pm 0.6^{t}$

0.11

6. $1 \pm 0.4^{9}$

0.20

$4.5 \pm 0.6^{h}$

0.28

$2.1 \pm 0.7$

Defatted ${ }^{3}$

$0.00^{1}$

$13.8 \pm 0.5$

0.05

$11.9 \pm 0.4^{k}$

0.10

$9.8 \pm 0.6$

0.17

$6.7 \pm 0.8^{\mathrm{m}}$

0.20

$4.6 \pm 0.5^{n}$

Parent starch treated with $\mathrm{NaOH}$ and $\mathrm{Na}_{2} \mathrm{SO}_{4}$ at $40^{\circ} \mathrm{C}$.

Heat-moisturetreated (16 h, $100 \% \mathrm{C} / 30 \%$ moisture).

Defatted with $75 \%$ propanol water.

"Means within a column with different superscripts are significantly different ( $p \leq 0.05)$. 
Table 4.14 Gelatinization parameters of hydroxypropyl native, defatted and heat-moisture treated starches

\begin{tabular}{|c|c|c|c|c|c|c|}
\hline \multirow{2}{*}{$\begin{array}{l}\text { Starch source and } \\
\text { treatment }\end{array}$} & \multirow{2}{*}{$\begin{array}{r}\text { Molar } \\
\text { substitution }\end{array}$} & \multicolumn{4}{|c|}{ Transition temperatures $\left({ }^{\circ} \mathrm{C}\right)$} & \multirow{2}{*}{$\begin{array}{l}\text { Enthalpy } \\
\Delta \mathrm{H}(\mathrm{J} / \mathrm{g})^{3}\end{array}$} \\
\hline & & $T_{0}{ }^{1}$ & $T_{p}{ }^{\prime}$ & $\mathrm{Te}^{\prime}$ & $T_{c}-T_{0}^{2}$ & \\
\hline Native & $\begin{array}{l}0.00^{4} \\
0.11 \\
0.18 \\
0.25\end{array}$ & $\begin{array}{l}54.5 \pm 0.2^{\mathrm{d}} \\
47.1 \pm 0.1^{\mathrm{b}} \\
44.0 \pm 0.2^{\mathrm{c}} \\
41.0 \pm 0.3^{\mathrm{d}}\end{array}$ & $\begin{array}{l}61.4 \pm 0.1^{\mathrm{a}} \\
56.2 \pm 0.4^{\mathrm{b}} \\
53.1 \pm 0.2^{\mathrm{c}} \\
52.0 \pm 0.2^{\mathrm{d}}\end{array}$ & $\begin{array}{l}66.4 \pm 0.6^{\mathrm{a}} \\
64.0 \pm 0.2^{\mathrm{b}} \\
63.0 \pm 0.1^{\mathrm{b}} \\
61.0 \pm 0.2^{\mathrm{c}}\end{array}$ & $\begin{array}{l}11.9 \pm 0.2^{\mathrm{a}} \\
16.9 \pm 0.3^{\mathrm{a}} \\
19.0 \pm 0.2^{\mathrm{b}} \\
20.0 \pm 0.3^{\mathrm{a}}\end{array}$ & $\begin{array}{l}10.9 \pm 0.2^{\circ} \\
9.6 \pm 0.2^{\mathrm{c}} \\
8.0 \pm 0.1^{\mathrm{d}} \\
7.4 \pm 0.2^{\mathrm{e}}\end{array}$ \\
\hline Heat-moisture treated ${ }^{5}$ & $\begin{array}{l}0.00^{4} \\
0.11 \\
0.20 \\
0.28\end{array}$ & $\begin{array}{l}63.0 \pm 0.0^{\circ} \\
54.0 \pm 0.3^{\prime} \\
52.0 \pm 0.3^{9} \\
47.3 \pm 0.4^{n}\end{array}$ & $\begin{array}{l}73.0 \pm 0.2^{\theta} \\
61.0 \pm 0.1^{\dagger} \\
60.1 \pm 0.4^{\theta} \\
59.8 \pm 0.2^{\theta}\end{array}$ & $\begin{array}{l}80.0 \pm 0.1^{\circ} \\
73.5 \pm 0.4^{\prime} \\
72.0 \pm 0.1^{\circ} \\
69.7 \pm 0.3^{n}\end{array}$ & $\begin{array}{l}17.0 \pm 0.4^{\theta} \\
19.5 \pm 0.2^{\prime} \\
20.0 \pm 0.6^{\prime} \\
22.4 \pm 0.2^{\circ}\end{array}$ & $\begin{array}{l}9.6 \pm 0.1^{\circ} \\
4.7 \pm 0.1^{\prime} \\
2.7 \pm 0.1^{\theta} \\
2.0 \pm 0.3^{h}\end{array}$ \\
\hline Defatted $^{6}$ & $\begin{array}{l}0.00^{4} \\
0.10 \\
0.18 \\
0.20\end{array}$ & $\begin{array}{l}58.0 \pm 0.4^{\prime} \\
51.0 \pm 0.7^{\prime} \\
49.5 \pm 0.1^{k} \\
48.5 \pm 0.2^{\prime}\end{array}$ & $\begin{array}{l}65.0 \pm 0.1^{\prime} \\
57.0 \pm 0.2^{\prime} \\
55.0 \pm 0.1^{k} \\
56.0 \pm 0.4^{\prime}\end{array}$ & $\begin{array}{l}72.5 \pm 0.3^{\prime} \\
68.0 \pm 0.2^{\prime} \\
69.0 \pm 0.2^{k} \\
69.5 \pm 0.2^{k}\end{array}$ & $\begin{array}{l}14.5 \pm 0.2^{\prime} \\
17.0 \pm 0.5^{k} \\
19.5 \pm 0.3^{\prime} \\
21.0 \pm 0.1^{\prime}\end{array}$ & $\begin{array}{l}15.9 \pm 0.3^{\prime} \\
11.1 \pm 0.2^{\prime} \\
9.2 \pm 0.2^{k} \\
8.4 \pm 0.1^{\prime}\end{array}$ \\
\hline
\end{tabular}

${ }^{1} T_{0}, T_{p}, \& T_{c}$ indicate respectively, the temperature of onset, mid point, and end of gelatinization.

${ }^{2}$ Gelatinization temperature range.

${ }^{3}$ Enthalpy of gelatinization.

${ }^{4}$ Parent starch treated with $\mathrm{NaOH}$ and $\mathrm{Na}_{2} \mathrm{SO}_{4}$ at $40^{\circ} \mathrm{C}$.

${ }^{5} \mathrm{Heat}-$ moisture treated $\left(30 \%\right.$ moisture, $\left.100^{\circ} \mathrm{C}, 16 \mathrm{~h}\right)$.

${ }^{6}$ Defatted with $75 \%$ n-propanol, $7 \mathrm{~h}$.

${ }^{a-1}$ Means within a column with different superscripts are significantly different ( $\left.p \leq 0.05\right)$. 
(Wootton \& Bamunuarachchi, 1979). At excess water content (as used in this study), potato starch develops only one endotherm ( $G$ transition). Donovan (1979) postulated that the $\mathrm{G}$ endotherm is due to the solvation assisted transition resulting from hydration and swelling of the amorphous region that facilitates the melting of crystallites during heating. The results indicate that the decrease in $T_{0 .}$ $T_{p}$ and $T_{c}$ on hydroxypropylation is due to disruption of the hydrogen bonds between starch chains in the amorphous regions by the hydroxypropyl groups. This increases the mobility of the starch chains which decreases the glass transition. $(\mathrm{Tg})$ temperature (Seow \& Thevamalar, 1993) and indirectly the melting temperature of starch crystallites.

The decrease in $\Delta \mathrm{H}$ on hydroxypropylation (Table 4.14) suggests that hydroxypropyl groups disrupt double helices (due to rotation of the flexible hydroxypropyl groups) within the amorphous regions of the granule. Consequently, the number of double helices that unravel and melt during gelatinization would be higher in unmodified than in hydroxypropylated starches. Due to crystalite disruption on heat-moisture treatment, the thermal motion of hydroxypropyl groups within the amorphous regions would be of a greater order of magnitude in heat-moisture treated than in native starch. Thus, at the same level of MS $(0.11)$, more double helices would be disrupted in heat-moisture treated than in native starch granule. Consequently, the number of double helices that unravel and melt during gelatinization would be lower (this represents a large decrease in $\Delta H$ ) in the former. 
The extent of decrease in $\Delta \mathrm{H}$ on hydroxypropylation should have been theoretically lower in defatted than in native starch due to the following reasons 1) lower degree of molar substitution in defatted starch and 2) increase in crystallinity on deffating (Fig. 4.2). However, as discussed earlier, crystallite reorientation occurs in both native and defatted (defatted $>$ native) starches (Fig.

4.3 and Table 4.7 ) on treatment (at $40^{\circ} \mathrm{C}$ ) with the alkaline reagents used during hydroxypropylation. Therefore, it can be postulated that after reorientation, the crystallites of defatted starch are not packed closely enough to significantly influence the mobility of the hydroxypropyl chains within the amorphous regions.

\subsubsection{Brabender viscosities (Pasting properties)}

The data in Table 4.15 summarizes the pasting characteristics of native, defatted and heat-moisture treated starches after hydroxypropylation. The pasting temperature of all starches decreased with increase in MS. Similar observations have been made on hydroxypropylated buffalo gourd (Butler et al. 1986) and pea starch (Hoover et al., 1988). Increases in the level of MS progressively increased the viscosity at $95^{\circ} \mathrm{C}$ for defatted starch (Table 4.15). However, for native and heat-moisture treated starches, an increase in the level of MS (beyond 0.18 and 0.20 , respectively), resulted in a decrease in viscosity at $95^{\circ} \mathrm{C}$. The changes in pasting temperature and viscosity with increase in MS levels are a reflection of the decrease in the strength of the associative bonding forces within the micellar network and to increases in SF (Table 4.12). The 
Table 4.15 Brabender viscosities (Pasting properties) of hydroxypropyl native, defatted and heat-moisture treated potato starches

\begin{tabular}{|c|c|c|c|c|c|}
\hline $\begin{array}{l}\text { Starch source \& } \\
\text { treatment }\end{array}$ & Molar substitution & $\begin{array}{c}\text { Pasting } \\
\text { temperature }\left({ }^{\circ} \mathrm{C}\right)\end{array}$ & $\begin{array}{c}\text { Viscosity at } 95^{\circ} \mathrm{C} \\
\left(\mathrm{BU}^{1}\right)\end{array}$ & $\begin{array}{l}\text { Viscosity after } 30 \\
\text { min at } 95^{\circ} \mathrm{C}\left(\mathrm{BU}^{1}\right)\end{array}$ & $\begin{array}{l}\text { Viscosity at } \\
50^{\circ} \mathrm{C}\left(\mathrm{BU}^{1}\right)\end{array}$ \\
\hline Native & $\begin{array}{l}0.00^{2} \\
0.11 \\
0.18 \\
0.25\end{array}$ & $\begin{array}{l}64.5 \pm 0.5^{a} \\
57.5 \pm 1.0^{b} \\
56.2 \pm 0.5^{b} \\
52.0 \pm 0.5^{c}\end{array}$ & $\begin{array}{c}750 \pm 10^{c} \\
1050 \pm 10^{b} \\
1200 \pm 15^{a} \\
760 \pm 10^{c}\end{array}$ & $\begin{array}{l}870 \pm 10^{a} \\
550 \pm 5^{b} \\
540 \pm 5^{b} \\
380 \pm 5^{d}\end{array}$ & $\begin{array}{c}1140 \pm 20^{a} \\
580 \pm 10^{c} \\
670 \pm 5^{b} \\
410 \pm 5^{d}\end{array}$ \\
\hline Heat-moisture treated ${ }^{3}$ & $\begin{array}{l}0.00^{2} \\
0.11 \\
0.20 \\
0.28\end{array}$ & $\begin{array}{l}91.5 \pm 0.5^{\natural} \\
79.5 \pm 0.5^{\prime} \\
67.5 \pm 1.0^{9} \\
60.0 \pm 1.0^{h}\end{array}$ & $\begin{array}{c}30 \pm 5^{h} \\
190 \pm 10^{9} \\
430 \pm 10^{\circ} \\
310 \pm 5^{\prime}\end{array}$ & $\begin{array}{c}130 \pm 10^{h} \\
250 \pm 15^{9} \\
580 \pm 10^{\circ} \\
340 \pm 5^{t}\end{array}$ & $\begin{array}{l}230 \pm 5^{h} \\
360 \pm 10^{9} \\
850 \pm 15^{\circ} \\
480 \pm 10^{f}\end{array}$ \\
\hline Defatted ${ }^{4}$ & $\begin{array}{l}0.00^{2} \\
0.10 \\
0.17 \\
0.20\end{array}$ & $\begin{array}{l}87.5 \pm 1.0^{\prime} \\
74.3 \pm 0.5^{\prime} \\
66.0 \pm 0.5^{k} \\
61.5 \pm 0.5^{1}\end{array}$ & $\begin{array}{l}330 \pm 5^{\prime} \\
900 \pm 15^{k} \\
1025 \pm 15^{\prime} \\
1300 \pm 10^{i}\end{array}$ & $\begin{array}{l}940 \pm 5^{m} \\
1090 \pm 10^{\prime} \\
1060 \pm 15 \\
870 \pm 10^{n}\end{array}$ & $\begin{array}{c}1460 \pm 10^{\prime} \\
1300 \pm 5^{m} \\
1210 \pm 10^{n} \\
940 \pm 10^{\circ}\end{array}$ \\
\hline
\end{tabular}

\footnotetext{
Brabender units:

${ }^{2}$ Parent starch treated with $\mathrm{NaOH}$ and $\mathrm{Na}_{2} \mathrm{SO}_{4}$ at $40^{\circ} \mathrm{C}$.

${ }^{3}$ Heat-moisture treated $\left(30 \%\right.$ moisture, $\left.100^{\circ} \mathrm{C}, 16 \mathrm{~h}\right)$.

${ }^{4}$ Defatted with $75 \%$ n-propanol, $7 \mathrm{~h}$.

a-o Means within a column with different superscripts are significantly different ( $p \leq 0.05$ ).
} 
decrease in the viscosity at $95^{\circ} \mathrm{C}$ seen at MS levels of 0.25 (native) and 0.28 (heat-moisture treated) can be attributed to the decrease in SF (at $90^{\circ} \mathrm{C}$ ) that occurs at the above MS levels for both native and heat-moisture treated starches (Table 4.12).

\subsection{STARCH DIGESTIBILITY BY PORCINE PANCREATIC $\alpha$-AMYLASE}

\subsubsection{Enzyme hydrolysis of native, defatted and heat-moisture treated} potato starches

The extent of hydrolysis of native, defatted and heat-moisture treated potato starches by porcine pancreatic $\alpha$-amylase at different time intervals are presented in Table 4.16. Both defatting and heat-moisture treatment increased (heat-moisture treatment > defatting ) the susceptibility of potato starch granules towards hydrolysis by $\alpha$-amylase. For instance, hydrolysis (at the end of $72 \mathrm{~h}$ ) increased by 25.6 and $17.3 \%$, on heat-moisture treatment and defatting (Table 4.16), respectively. It has already been shown that crystalline regions of potato starch granules disrupt on heat-moisture treatment. Consequently, the amount of amorphous regions available for enzyme hydrolysis would increase after heatmoisture treatment. This would then explain the difference in reactivity of native and heat-moisture treated starches towards enzyme hydrolysis. It has also been shown that defatting increases the crystallinity of potato starch granules. On this basis, defatted granules should have been less susceptible than native granules 


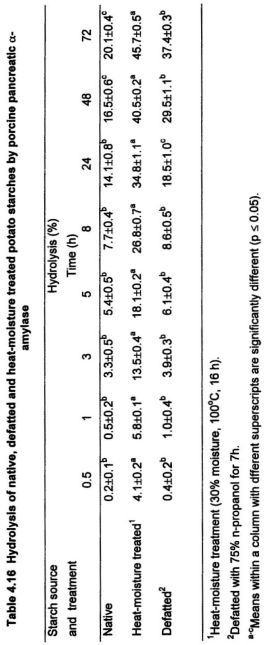


towards hydrolysis by $\alpha$-amylase. Vasanthan \& Hoover (1992a) have shown that amylose chains entrapped within adjacent chain clusters of amylopectin are released into the amorphous regions on defatting. Thus, more amylose chains would be available for $\alpha$-amylase action in defatted than in native granules. This would then explain the increase in hydrolysis on defatting.

The mode of attack by $\alpha$-amylase on native, defatted and heat-moisture treated starches was investigated by scanning electron microscopy (Figs. 4.6a-f). The attack of $\alpha$-amylase (72 h) on native potato starch manifested itself in only superficial surface erosion of the granules (Figs. 4.6a,b). During the same time period, granules of defatted (Figs4,6c,d) and heat-moisture treated (Figs. 4.6e,f) starches were more extensively attacked by $\alpha$-amylase than those of native starch (Figs. 4.6a,b). The surfaces of both defatted and heat-moisture treated starches were extensively eroded and were covered with numerous pits of varying size. ranule splitting was more evident in heat-moisture treated than in defatted granules.

The influence of time of heat-moisture treatment and defatting on the susceptibility of potato starch granules towards $\alpha$-amylase hydrolysis (24 h) are presented in Tables 4.17 and 4.18 respectively. Heat-moisture treatment for 1,3 , 6 and $8 \mathrm{~h}$, respectively, increased hydrolysis by 17.6, 21.8, 24.8 and $29.1 \%$. However, the above trend was not observed in granules which were heat- 
Fig. 4.6 Scanning electron micrographs of native (A, B), defatted (C, D), and heat-moisture treated (E. F) potato starch granules hydrolyzed by porcine pancreatic $\alpha$-amylase for $72 \mathrm{~h}$. 


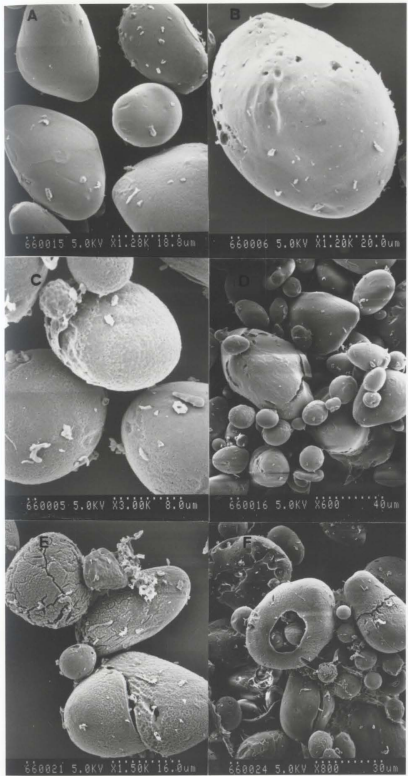


Table 4.17 Effect of time of heat-moisture treatment on hydrolysis of potato starch by porcine pancreatic $\alpha$-amylase

\begin{tabular}{cc}
\hline $\begin{array}{c}\text { Time of heat-moisture treatment } \\
(\mathrm{h})\end{array}$ & Hydrolysis $^{2}(\%)$ \\
\hline Control (native potato starch) & $14.1 \pm 0.8^{\mathrm{g}}$ \\
1 & $31.7 \pm 1.2^{\mathrm{e}}$ \\
3 & $35.9 \pm 0.5^{\mathrm{da}}$ \\
6 & $38.9 \pm 0.8^{\mathrm{b}}$ \\
8 & $43.5 \pm 1.1^{\mathrm{a}}$ \\
10 & $37.5 \pm 1.2^{\mathrm{bc}}$ \\
16 & $34.8 \pm 1.1^{\mathrm{d}}$ \\
30 & $28.6 \pm 1.0^{\mathrm{f}}$ \\
\hline
\end{tabular}

\footnotetext{
'Heat-moisture treatment $\left(30 \%\right.$ moisture, $\left.100^{\circ} \mathrm{C}\right)$.

${ }^{2}$ Hydrolysis with $\alpha$-amylase (12 units / mg starch, 24 h).

a-geans within the same column with different superscripts are significantly different ( $p$ $\leq 0.05$ ).
} 
Table 4.18 Effect of time of defatting on hydrolysis of potato starch by porcine pancreatic $\alpha$-amylase

\begin{tabular}{cc}
\hline Time of defatting $^{\prime}(\mathrm{h})$ & Hydrolysis $^{\mathrm{C}}(\%)$ \\
\hline Control (native potato starch) & $14.1 \pm 0.8^{\mathrm{c}}$ \\
1 & $13.4 \pm 0.9^{\mathrm{d}}$ \\
2 & $13.6 \pm 0.6^{\mathrm{da}}$ \\
4 & $14.8 \pm 0.8^{\mathrm{c}}$ \\
7 & $18.5 \pm 1.0^{\mathrm{b}}$ \\
9 & $20.8 \pm 0.6^{\mathrm{a}}$ \\
12 & $12.6 \pm 0.4^{\mathrm{d}}$
\end{tabular}

\section{'Defatting with $75 \%$ n-propanol.}

${ }^{2}$ Hydrolysis with $\alpha$-amylase (12 units / $\mathrm{mg}$ starch, $24 \mathrm{~h}$ ).

${ }^{2-d}$ Means within the same column with different superscripts are significantly different $(p \leq 0.05)$. 
moisture treated for periods exceeding $8 \mathrm{~h}$. For instance, after 10,16 and $30 \mathrm{~h}$, the increase in hydrolysis was $23.4,20.7$ and $14.5 \%$, respectively (Table 4.17). Significant differences in hydrolysis between native and defatted starches were observed only after $7 \mathrm{~h}$ defatting (Table 4.18). Hydrolysis increased by 5.1 and $6.4 \%$, respectively, in starches defatted for 7 and $9 \mathrm{~h}$ (Table 4.18). However, potato starch defatted for $12 \mathrm{~h}$ was hydrolyzed to a lesser extent $(12.6 \%)$ than native starch (14.1\%) [Table 4.18].

As discussed earlier, crystallite destruction occurs on heat-moisture treatment. Consequently, more amorphous regions are made available for $\alpha$ amylase action than in native starch. This would then explain the progressive increase in hydrolysis during the first $8 \mathrm{~h}$ of heat-moisture treatment (Table 4.17).

Hoover \& Vasanthan (1994a) have shown that in potato starch, AM-AM and AM-AMP interactions occur during heat-moisture treatment $\left(100^{\circ} \mathrm{C}, 30 \%\right.$ moisture, $16 \mathrm{~h}$ ). The difference in enzyme susceptibility between heat-moisture treated starches for periods greater than $8 \mathrm{~h}$, and heat-moisture treated starches for less than $8 \mathrm{~h}$ (Table 4.17 ) suggests that heat-moisture treatment for periods greater than $8 \mathrm{~h}$ induces interactions to occur between AM-AM and AM-AMP chains. These interactions lead to the formation of new crystallites which reduce the accessibility of $\alpha$-amylase to the glycosidic linkages of the starch chains. The data also suggests that crystallite disruption does not occur to any significant extent beyond $8 \mathrm{~h}$ of heat-moisture treatment. 
The X-ray intensity of the peak centred at 5.2 A (Table 4.19 and Fig. 4.7). also suggests that new crystallites begin to form only after $8 \mathrm{~h}$ of heat-moisture treatment. For instance, at 1, 8, 16 and $30 \mathrm{~h}$ of heat-moisture treatment, the intensity of the $5.2 \AA$ peak, was $1674,1575,2105$ and 2809 cps, respectively. The reduction in peak intensity at $8 \mathrm{~h}$, indicates crystallite disruption.

Amylose complexed with lipids has been shown to significantly reduce the availability to $\alpha$-amylase in vitro (Holm et al., 1983; Biliaderis et al., 1985). The results (Table 4.18) indicate that the increase in hydrolysis on defatting could be due to the interplay of two factors : (1) lipid removal and (2) the availability of more free amylose chains for $\alpha$-amylase action (Vasanthan \& Hoover, 1992a). This seems plausible since granule susceptibility to $\alpha$-amylase continues to increase (Table 4.18) even after complete lipid removal (lipid removal is completed after $7 \mathrm{~h}$ of defatting [Fig. 4.8]). Defatting beyond $9 \mathrm{~h}$ decreases hydrolysis (Table 4.18) possibly due to AM-AM, AM-AMP and AMP-AMP interactions. The progressive change in X-ray diffraction patterns during defatting (0-12 h) [Fig. 4.9] may also reflect that changes occurred in the crystalline pattern of the starch granule. These interactions probably result in the formation of double helices which are resistant to $\alpha$-amylase action. Thoma (1968) postulated that the enzyme catalyzed hydrolysis of the $\alpha-D(1 \rightarrow 4)$ glycosidic bond of the starch molecule involves enzyme induced ring distortion of one of the D-glucosyl residues from the ${ }^{4} \mathrm{C}_{1}$ "chair' conformation to a "half chair" 
Table 4.19 X-raydiffraction intensities of the $5.2 \mathrm{~A}$ peak with time course of heat-moisture treatment

\begin{tabular}{cc}
\hline $\begin{array}{c}\text { Time of heat-moisture } \\
\text { treatment }{ }^{1}(\mathrm{~h})\end{array}$ & $\begin{array}{c}\text { X-ray intensities of the peak } \\
\text { centered at } 5.2 \AA(\mathrm{cps})^{2}\end{array}$ \\
\hline 1 & 1674 \\
8 & 1575 \\
16 & 2105 \\
30 & 2809
\end{tabular}

\footnotetext{
${ }^{1}$ At $100^{\circ} \mathrm{C}, 30 \%$ moisture.

${ }^{2}$ Counts per second.
} 
Fig. 4.7 X-ray diffraction patterns of potato starches heat-moisture treated for (A) $0 \mathrm{~h},(\mathrm{~B}) 1 \mathrm{~h}$, (C) $8 \mathrm{~h}$, (D) $16 \mathrm{~h}$ and (E) 30h. 


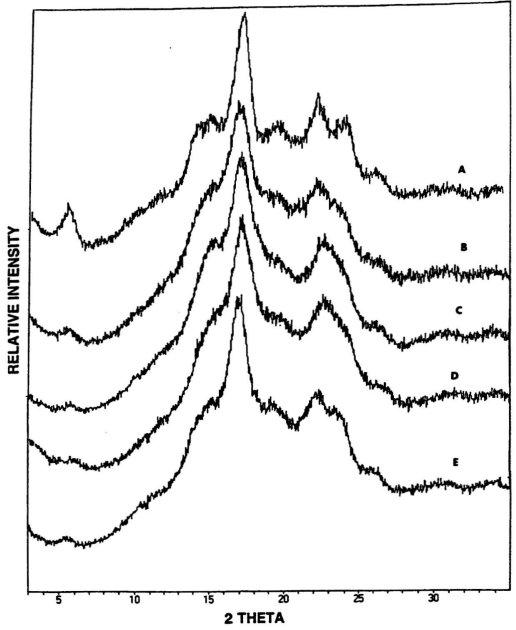


Fig. 4.8 The influence of defatting time on the extent of lipid removal from native potato starch. 


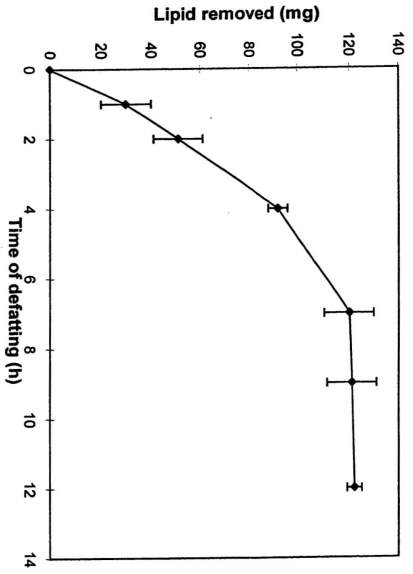


Fig. 4.9 X-ray diffraction patterns of potato starched defatted for (A) $0 \mathrm{~h}$, (B) 1 h, (C) $7 \mathrm{~h}$, and (D) $12 \mathrm{~h}$. 


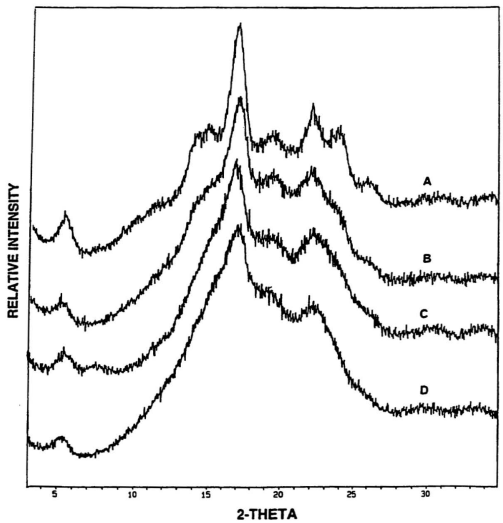


conformation (Fig 2.28b). This ring distortion decreases the enthaipy of activation and increases the susceptibility of the glucosyl residues to nucleophilic attack by functional groups on the enzyme and water. Laszlo et al. (1978) have shown that ring distortion or a "half chair' conformation is involved in the transition state of $\alpha$-amylase. It is therefore plausible that conformational changes (chair-half chair) during $\alpha$-amylase hydrolysis may be difficult for those amylose chains that form double helices during defatting. This would then explain the decrease in hydrolysis when defatting is continued beyond $9 \mathrm{~h}$. This decrease in hydrolysis cannot be due to crystallite formation (resulting from aggregation of the newly formed double helices) since X-ray diffraction intensities did not change during the time course of defatting (not shown).

\subsubsection{Influence of alkali on enzyme hydrolysis of native, defatted and heat- moisture treated potato starches}

The influence of the alkaline conditions used during hydroxypropylation on enzyme digestibility of native, defatted $(7 \mathrm{~h})$ and heat-moisture treated $(30 \%$ moisture, $100^{\circ} \mathrm{C}, 16 \mathrm{~h}$ ) potato starches are presented in Table 4.20 . Alkaline treatment increased the susceptibility of the above starches (native > defatted > heat-moisture treated) towards hydrolysis by $\alpha$-amylase. For instance after $24 \mathrm{~h}$, hydrolysis was increased by $20.0,0.7 \%$ and $12.2 \%$, respectively, in alkali treated native, heat-moisture treated and defatted starches (Table 4.20). 
Table 4.20 Hydrolysis of native, defatted and heat-moisture treated potato starches by porcine pancreatic $\alpha$-amylase before and after hydroxypropylation

\begin{tabular}{|c|c|c|c|}
\hline $\begin{array}{l}\text { Starch source and } \\
\text { treatment }\end{array}$ & $\begin{array}{l}\text { Propylene oxide } \\
\text { added (\%v/w) }\end{array}$ & $\begin{array}{c}\text { Molar } \\
\text { substitution }\end{array}$ & Hydrolysis' (\%) \\
\hline Native & - & - & $14.1 \pm 0.8^{d}$ \\
\hline Native (alkali treated) ${ }^{2}$ & 0 & 0.00 & $34.1 \pm 0.3^{c}$ \\
\hline & 2 & 0.05 & $33.5 \pm 1.1^{c}$ \\
\hline & 5 & 0.11 & $35.4 \pm 1.0^{c}$ \\
\hline & 10 & 0.18 & $39.7 \pm 1.1^{b}$ \\
\hline & 15 & 0.25 & $44.5 \pm 0.9^{a}$ \\
\hline & 20 & 0.29 & $39.0 \pm 0.9^{b}$ \\
\hline Heat-moisture treated $(\mathrm{HMT})^{3}$ & $\cdot$ & - & $34.8 \pm 1.1^{\mathrm{h}}$ \\
\hline \multirow[t]{6}{*}{ HMT (alkali treated) ${ }^{2}$} & 0 & 0.00 & $35.5 \pm 1.0^{g h}$ \\
\hline & 2 & 0.05 & $37.5 \pm 0.9^{6}$ \\
\hline & 5 & 0.11 & $37.9 \pm 1.1^{\prime}$ \\
\hline & 10 & 0.20 & $44.4 \pm 1.0^{e}$ \\
\hline & 15 & 0.28 & $30.5 \pm 0.4^{1}$ \\
\hline & 20 & 0.34 & $26.2 \pm 1.0^{\prime}$ \\
\hline Defatted $^{4}$ & - & $\cdot$ & $18.5 \pm 1.0^{\circ}$ \\
\hline \multirow[t]{6}{*}{ Defatted (alkali treated) ${ }^{2}$} & 0 & 0.00 & $30.7 \pm 0.3^{n}$ \\
\hline & 2 & 0.05 & $38.2 \pm 0.7$ \\
\hline & 5 & 0.10 & $39.7 \pm 0.5^{1}$ \\
\hline & 10 & 0.17 & $42.3 \pm 0.7^{k}$ \\
\hline & 15 & 0.20 & $43.1 \pm 0.9^{k}$ \\
\hline & 20 & 0.26 & $35.5 \pm 0.6^{m}$ \\
\hline
\end{tabular}

${ }^{1} \mathrm{Hydrolysis}$ with $\alpha$-amylase for $24 \mathrm{~h} / 12$ units/mg starch.

${ }^{2}$ Parent starch treated with $\mathrm{NaOH}$ and $\mathrm{Na}_{2} \mathrm{SO}_{4}$ at $40^{\circ} \mathrm{C}$.

${ }^{3}$ Heat-moisture treatment $\left(16 \mathrm{~h} / 100^{\circ} \mathrm{C} / 30 \%\right.$ moisture).

${ }^{4}$ Defatted with $75 \%$ propanol for $7 \mathrm{~h}$.

${ }^{-o}$ Means within a column with different superscripts are significantly different ( $p \leq$ 0.05 ). 
The increase in hydrolysis on alkaline treatment can be attributed to the interplay of three factors : 1) disruption of double helices (within the amorphous regions) in the presence of the alkaline reagents. (This would provide $\alpha$-amylase greater accessibility to the glycosidic linkages); 2) Ionization of the hydroxyl groups on adjacent starch chains which results in a partial separation of protons leaving the core of the starch negatively charged (repulsion between negatively charged starch chains would increase hydrolysis, by increasing the degree of accessibility to $\alpha$-amylase); and 3 ) an increase in granular solubility resulting from aggregation of water dipoles around the negatively charged centres. Double helices within the amorphous regions have been shown to disrupt during heatmoisture treatment. Consequently, fewer double helices would be present in heat-moisture treated starch than in native and defatted starches. Thus, the number of double helices that disrupt during alkali treatment would be of a lower order of magnitude in heat-moisture treated than in native and defatted starch granules. This would then explain the smaller increase in $\alpha$-amylase hydrolysis after alkaline treatment of heat-moisture treated starch. The different responses shown by alkali treated native and defatted starches towards hydrolysis by $\alpha$ amylase can de attributed to the greater degree of crystallinity (a higher degree of crystallinity would hinder the penetration of the alkaline reagents into the granule interior) inherent within granules of defatted starch (Vasanthan \& Hoover, 1992a). This would then explain the observed increase in hydrolysis 
(native > defatted > heat-moisture treated) after alkaline treatment. The influence of factors 2 and 3 on hydrolysis is probably of the same order of magnitude in all three starches.

\subsubsection{The Influence of the level of hydroxypropylation on enzyme hydrolysis} of native, defatted and heat-moisture treated starches

The susceptibility of native, heat-moisture treated and defatted potato starches towards hydrolysis by $\alpha$-amylase increased with increase in MS levels. However, granular susceptibility began to decrease at MS levels of 0.29 (native), 0.28 (heat-moisture treated) and 0.26 (defatted) [Table 4.20].

Studies on the effect of varying MS levels on hydrolysis of hydroxypropylated starches have been conducted on wheat starch (Wootton \& Chaudhry, 1981), maize, waxy maize and high amylose maize starches (Mohd Azemi \& Wootton, 1985; 1995; Hahn \& Hood, 1980), potato starch (Leegwater \& Luten ,1971) and pea starch (Hoover et al., 1988). Wootton \& Chaudhry (1981) showed that an increase in MS $(0.03 \rightarrow 0.17)$ increased the digestibility of raw wheat starch by pancreatic $\alpha$-amylase. However, a similar increase in MS for gelatinized wheat starch decreased its digestibility. The above authors postulated that the increased digestibility of raw starches with higher MS is probably due to the greater tendency of more highly substituted starches to swell in cold water (This would allow better access of the enzyme to the substrate). 
However, the reduction in digestibility of gelatinized starch with increase in MS was attributed to greater reduction of enzyme attack by increasing number of substituent groups. Mohd Azemi \& Wootton (1985) showed that hydrolysis of gelatinized normal, waxy and high amylose maize starches decreased with increase in the level of MS $(0.05 \rightarrow 0.13)$. A similar increase in MS levels for raw normal and high amylose maize starches, resulted in decrease in digestibility at lower MS levels, followed by increases at higher MS levels (> 0.07). However, the digestibility of raw waxy maize starch showed a continuing drop as MS increased. Hahn \& Hood (1980) showed that dimethyl sulphoxide solubilized hydroxypropylated waxy maize starch was hydrolyzed to a lesser extent than its unmodified counterpart. However, the extent of hydrolysis was not influenced by increases in MS. Leegwater \& Luten (1971) showed that the digestibility of gelatinized potato starch by large amounts of pancreatin decreased exponentially with increase in the degree of substitution $(0.02 \rightarrow 0.45)$. Hoover et al. (1988) showed that the digestibility of raw field pea starch decreased with increase in MS up to a level of 0.08 . Further increases in MS caused an increase in digestibility which was higher than that observed for native starch. 


\subsection{RETROGRADATION STUDIES ON POTATO STARCHES}

\subsubsection{Turbidimetric and microstructural analysis}

\subsubsection{Initial turbidity and scanning electron microscopy of fresh pastes of}

native, defatted and heat-moisture treated potato starch pastes

Turbidity development in native, defatted and heat-moisture treated potato starch pastes during $24 \mathrm{~h}$ of storage at $4^{\circ} \mathrm{C}$ is described in Figs. 4.10a, 4.11a and 4.12a. The initial turbidity of the gelatinized starch pastes followed the order : heat-moisture treated (Fig. 4.12a) > defatted (Fig. 4.11a) > native (Fig. 4.10a). The pellet of freshly gelatinized native potato starch paste was devoid of granule remnants and consisted of large sheets of leached amylose and amylopectin (Fig 4.13a) [Jacobson et al., 1997]. However, fresh pastes of defatted (Fig 4.13c) and heat-moisture treated (Fig 4.13e) starches consisted of granule remnants (heat-moisture treated $>$ defatted) connected by a matrix of leached amylose and amylopectin.

Turbidity effects have their origin in refractive index fluctuation over a distance scale comparable to the wavelength of observation. In a polymersolvent system this is caused by density fluctuations over the same distance scale and is most likely due to extensive polymer-polymer aggregation (Gidley \& Bulpin, 1989). Gidley \& Bulpin (1989) have shown, on the basis of their studies of amylose aggregation in aqueous systems, that even at the onset of detectable turbidity, highly aggregated polymer structures are present. Craig et al. (1989) have classified starch pastes into three categories depending on the behaviour in 
Fig. 4.10 Time course of turbidity development in native potato starch stored at $4^{\circ} \mathrm{C}(\mathrm{A})$ and $40^{\circ} \mathrm{C}(\mathrm{B})$.

- native; alkali treated native (MS 0.00); $\boldsymbol{\Delta}$ hydroxypropylated native (MS 0.11 ); $\mathbf{X}$ hydroxypropylated native (MS 0.18); * hydroxypropylated native (MS 0 . 25). 

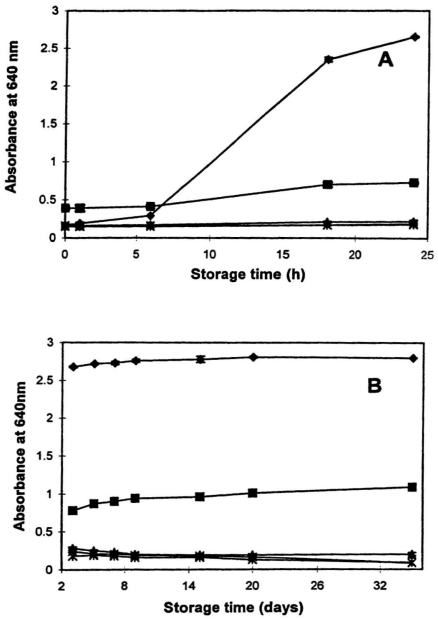
Fig. 4.11 Time course of turbidity development in defatted potato starch stored at $4^{\circ} \mathrm{C}(\mathrm{A})$ and $40^{\circ} \mathrm{C}(\mathrm{B})$.

- defatted; $\mathbf{a l k a l i}$ treated defatted (MS 0.00); $\boldsymbol{\Delta}$ hydroxypropylated defatted (MS 0.10), X hydroxypropylated defatted (MS 0.17), * hydroxypropylated defatted (MS 0.20). 

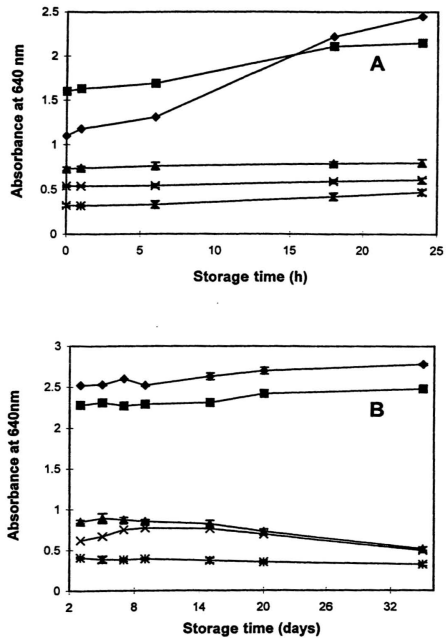
Fig. 4.12 Time course of turbidity development in heat-moisture treated potato starch stored at $4^{\circ} \mathrm{C}(\mathrm{A})$ and $40^{\circ} \mathrm{C}(\mathrm{B})$.

- heat-moisture treated; alkali treated heat-moisture treated (MS 0.00); hydroxypropylated heat-moisture treated (MS 0.11); $\mathbf{X}$ hydroxypropylated heat-moisture treated (MS 0.20); * hydroxypropylated heat-moisture treated (MS 0.28). 

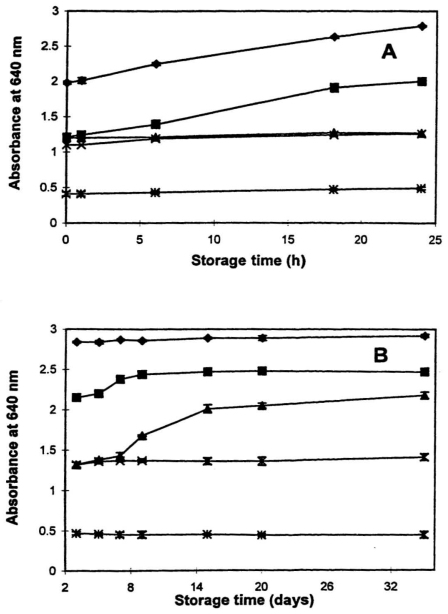
Fig. 4.13 Scanning electron micrographs of freshly gelatinized and stored ( $24 \mathrm{~h}$ at $4^{\circ} \mathrm{C}$ ) potato starch pastes : (A) fresh native potato stash, (B) stored native potato starch, (C) fresh defatted potato starch, (D) stored defatted potato starch, (E) fresh heat-moisture treated potato starch, (F) stored heat-moisture treated potato starch. 


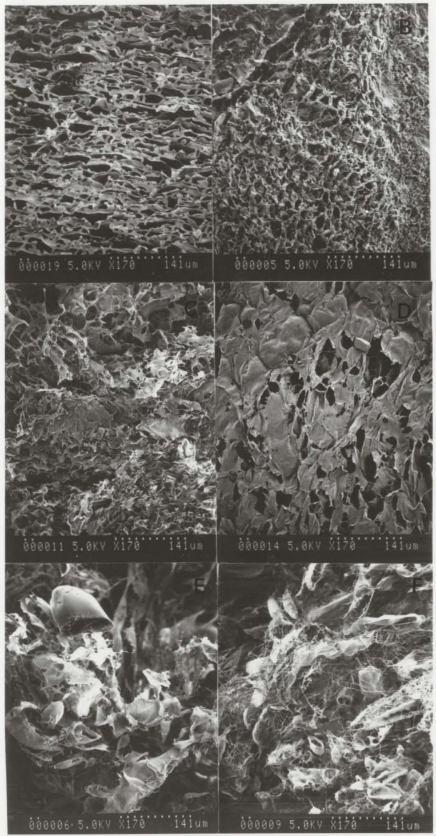


Fig. 4.14 Scanning electron micrographs of gelatinized and stored $\left(24 \mathrm{~h} \mathrm{at} 4^{\circ} \mathrm{C}\right.$ ) alkali treated native potato starch pastes. (A) \& (B) fresh paste; (C) \& (D) stored paste. 


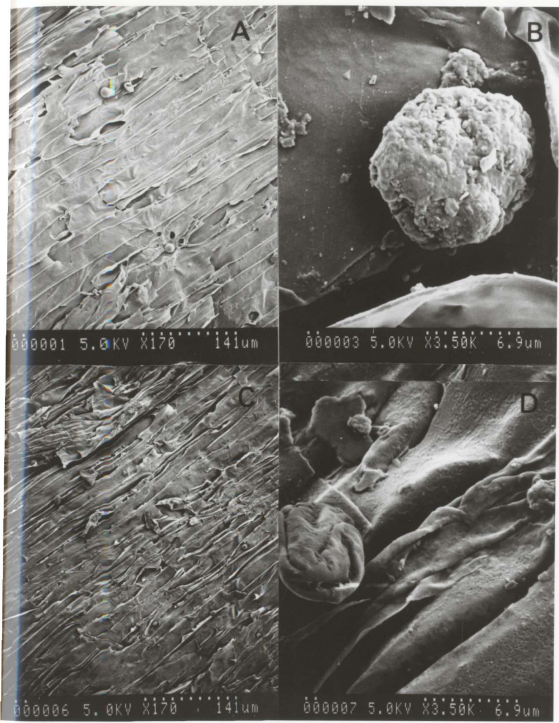


Fig. 4.15 Scanning electron micrographs of freshly gelatinized and stored ( $24 \mathrm{~h}$ at $40^{\circ} \mathrm{C}$ ) pastes of alkali treated defatted potato starch : (A) \& (B) freshly gelatinized defatted starch, (C) \& (D) stored defatted starch. 


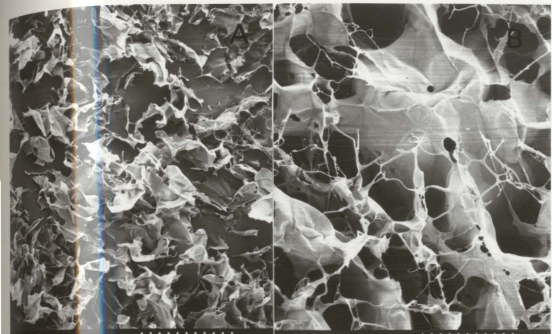

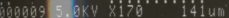

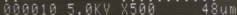

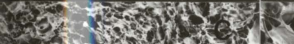

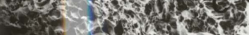

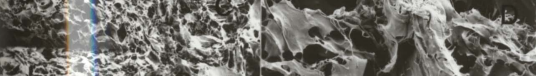

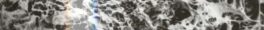

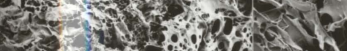

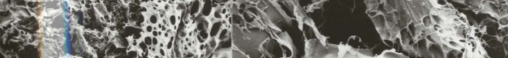
W.

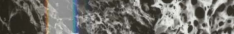

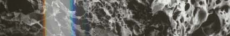

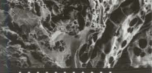

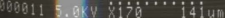


Fig. 4.16 Scanning electron micrographs of freshly gelatinized and stored ( $24 \mathrm{~h}$ at $4^{\circ} \mathrm{C}$ ) starch pastes of alkali treated heat-moisture treated potato starch : (A) \& (B) freshly gelatinized heat-moisture treated starch, (C) \& (D) stored heatmoisture treated starch. 


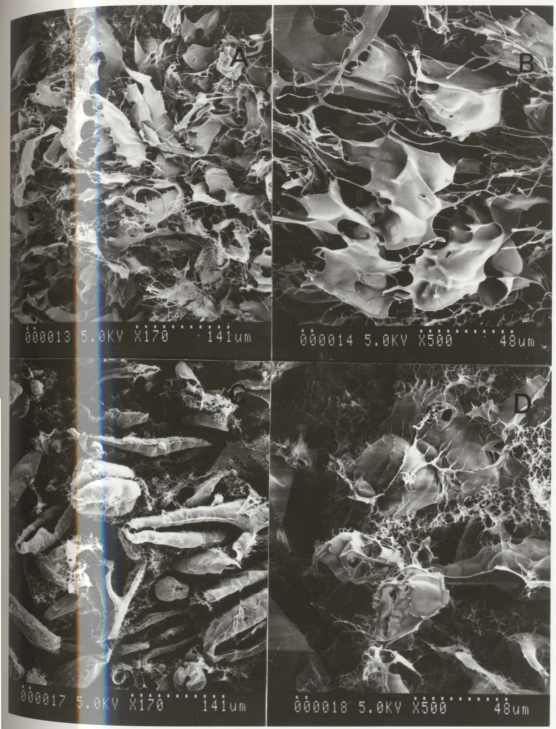


decrease in light absorbance (in spite of an increase in the amount of granule remnants) on alkaline treatment of heat-moisture treated starch (Fig. 4.12a) is probably due to decreased interaction between leached amylose and/or amylopectin chains (compare freshly gelatinized heat-moisture treated gel [Fig. 4.13e] and freshly gelatinized alkali treated heat-moisture treated gel [Figs. 4.16a,b]).

4.7.1.3 Initial turbidity and scanning electron micrographs of hydroxypropylated native, defatted and heat-moisture treated starches

The initial absorption of alkali treated starches decreased on hydroxypropylation (Figs. 4.10a, 4.11a, 4.12a). In alkali treated native starch, absorption decreased by $58.9 \%$ at MS levels of 0.11 and 0.18 , whereas, at MS 0.25 the corresponding value was $61.2 \%$ (Fig $4.10 \mathrm{a}$ ). In alkali treated defatted starch, absorption decreased by $54.3,66.2$ and $80 \%$, respectively, at MS 0.10 , 0.17 and 0.20 (Fig. 4.11a). Whereas, in alkali treated heat-moisture treated starch, absorption decreased by $2.4,10.0,66.6 \%$, respectively, at MS $0.11,0.20$ and 0.28 (Fig. 4.12a).

Scanning electron micrographs of freshly gelatinized pastes of native, defatted and heat-moisture treated starches after hydroxypropylation (under alkaline conditions) are presented in Fig. 4.17. Granules of all starches were disrupted on hydroxypropylation. The disrupted granules of hydroxypropylated (MS 0.11) native starch (Fig. 4.17a) and hydroxypropylated (MS 0.10) defatted 
Fig. 4.17 Scanning electron micrographs of freshly gelatinized and stored ( $24 \mathrm{~h}$ at $4{ }^{\circ} \mathrm{C}$ ) pastes of hydroxypropylated potato starches : (A) fresh hydroxypropylated (MS 0.11) native starch; (B) stored hydroxypropylated (MS 0.11) native starch; (C) fresh hydroxypropylated (MS 0.10) defatted starch; (D) stored hydroxypropylated (MS 0.10) defatted starch; (E) \&(F) fresh hydroxypropylated (MS 0.11) heat-moisture treated starch; (G) \& (H) stored hydroxypropylated (MS 0.11 ) heat-moisture treated starch. 
0.212

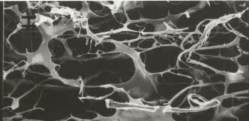

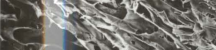

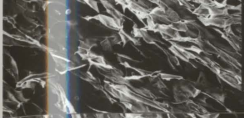

Bge

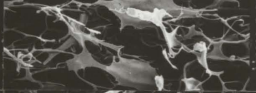

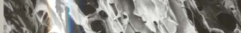

(M)

Whe bre

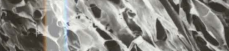

S.M.

$\frac{1}{4}$

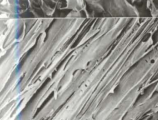

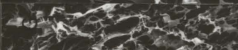

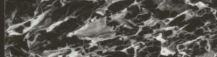

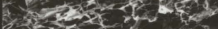

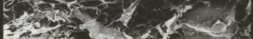

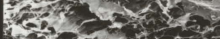

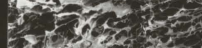
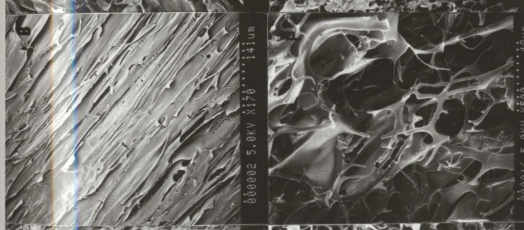

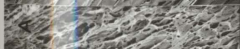

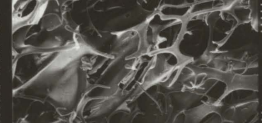

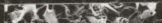

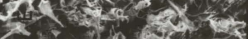

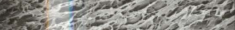

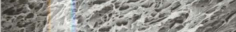

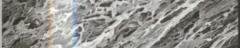

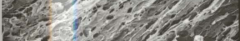

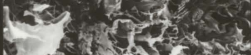

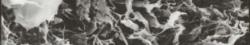
in

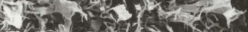

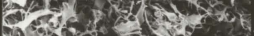
Wh 5 . If 
starch (Fig. 4.17c) were in the form of elongated sheets connected by a network of amylose and amylopectin exudates. The morphology of the granule remnants of both freshly gelatinized native (Fig. 4.17a) and defatted (Fig. 4.17c) starches after hydroxypropylation were different from those of their unmodified counterparts [Fig. 4.13a (native), Fig. 4.13c (defatted)]. In comparison with hydroxypropylated native and defatted starches, the extent of granule disruption was less marked in hydroxypropylated (MS 0.11) heat-moisture treated starch (Fig. 4.17e,f). Furthermore, there were many small intact granules (Fig. 4.17e) which seemed to have escaped disruption during hydroxypropylation.

The hydroxypropyl groups introduced into the starch chains are said to be capable of destroying inter- and intramolecular hydrogen bonds, thereby weakening the granular structure (Wootton \& Manatsathit, 1984). Kim et al. (1992) have shown, using light microscopy, that the central region of the hydroxypropylated potato starch granules become more and more disrupted with an increase in MS.

The results on hydroxypropylated native starch (Fig. 4.10a, Fig. 4.17a) suggests that although granule stability increases after alkaline treatment (Fig. 4.14a,b), the extent of this increase is not large enough to prevent granule breakdown (Fig. 4.17a) when hydroxypropylated native starch (MS 0.11) is heated under the conditions used for turbidity measurements. Thus, the decrease in initial absorbance at MS 0.11 is due to a sharp decrease in the amount of granule structure present in the starch paste. This would also explain 
the lack of influence of increasing MS (0.11-0.25) on light absorbance (Fig. 4.10a).

The progressive decrease in absorbance with increase in MS for defatted (Fig. 4.11a) and heat-moisture treated (Fig. 4.12a) starches suggests that granule remnants of these starches are better able to resist (heat-moisture treated > defatted) the disruptive action of hydroxypropyl groups on granule structure than those of native starch. This can be attributed to the interactions that occur between starch chains on defatting (Vasanthan \& Hoover, 1992a) and heat-moisture treatment (Hoover \& Vasanthan, 1994a). At similar MS levels (0.20), the absorption decrease is higher in defatted (66\%) [Fig. 4.11a] than in heat-moisture treated (10\%) starch [Fig. 4.12a] due to greater stability of heatmoisture treated granules. The steep decrease in absorbance $(66.2 \%)$ at MS 0.28 for heat-moisture treated starch (Fig. 4.12a) suggests that at this MS level granules undergo extensive disruption into small fragments.

\subsubsection{Turbidity development and scanning electron micrographs of native,} defatted and heat-moisture treated starch pastes during storage

The development of turbidity during storage of the above starches at $4^{\circ} \mathrm{C}$ (1 day) followed by storage at $40^{\circ} \mathrm{C}$ for 34 days are presented in Figs. 4.10, 4.11 and 4.12. In all starches absorbance increased with storage time. The extent of this increase was more pronounced (native > defatted $>$ heat-moisture treated) during the first day of storage (Figs. 4.10a, 4.11a, 4.12a). The absorbance 
increase between $1^{\text {st }}$ and $35^{\text {th }}$ day of storage followed the order : defatted (Fig. 4.11b) > native (Fig. 4.10b) > heat-moisture treated (Fig. 4.12b).

Scanning electron micrographs of the starch pastes after storage for $24 \mathrm{~h}$ (at $4^{\circ} \mathrm{C}$ ) are presented in Fig. $4.13 \mathrm{~b}, \mathrm{~d}, \mathrm{f}$. The microstructure of the stored pastes of native starch consisted of a dense network formed by leached amylose and amylopectin chains (Fig. 4.13b), whereas, that of defatted starch (Fig. 4.13d) consisted of swollen granule remnants enmeshed and connected together by thick strands of the leached exudate. Heat-moisture treated granule remnants were enmeshed and held together by a network formed by thin strands of the leached exudate (Fig. 4.13f). Many researchers (Craig et al., 1989; Bello-perez \& Paredes-Lopez, 1996; Jacobson et al., 1997) have shown that turbidity development during storage is influenced by factors such as granule swelling, granule remnants, leached amylose and amylopectin , amylose and amylopectin chain lengths, intra or intermolecular bonding, lipids, sucrose, cross-linking and substitution.

The first stage of retrogradation has been shown to involve mainly amylose aggregation and crystallization which is completed within the first few hours of storage. However, the second stage that occurs over a longer periods (days) involves mainly amylopectin aggregation and crystallization (Miles et al., 1985, Goodfellow \& Wilson, 1990; Silverio et al., 1996).

The rapid increase in turbidity during the first $24 \mathrm{~h}$ of storage was thus, mainly due to continued interaction (via hydrogen bonding) between leached 
amylose chains and to a lesser extent short range ordering of amylopectin side chains. These interactions would lead to the development of junction zones, which would reflect or scatter a significant amount of light.

As shown before, the extent of amylose leaching during gelatinization is more pronounced in native than in defatted or heat-moisture treated starches (defatted > heat-moisture treated). Thus, the magnitude of interaction between leached amylose chains during storage would follow the above trend. This would then partially explain the extent of increase in absorbance [native (Fig. 4.10a) > defatted (Fig. 4.11a) $>$ heat-moisture treated (Fig. 4.12a)] during the first $24 \mathrm{~h}$ of storage. It is likely that the magnitude of the increase in absorbance during the $24 \mathrm{~h}$ storage period is also influenced by the presence of granule remnants in starch pastes of defatted (Fig. 4.13c) and heat-moisture treated (Fig. 4.13e) starches (heat-moisture treated $>$ defatted). These remnants would hinder chain aggregation during storage. The slow increase in turbidity beyond $24 \mathrm{~h}$ storage (Figs. 4.10b, 4.11b, 4.12b) can be attributed to slow interaction between the outer branches of amylopectin chains (Ring et al., 1987) of native, defatted and heat-moisture treated starches. Vasanthan \& Hoover (1992a) have shown that defatting increases crystallinity, whereas heat-moisture treatment disrupts crystallinity in potato starch. Consequently, the degree of separation (after gelatinization) between the outer branches of amylopectin chains would be much greater in heat-moisture treated than in defatted starch. Thus, during storage, the formation and lateral association of double helices involving amylopectin 
chains would be easier and much stronger in defatted than in heat-moisture treated or native starch (native > heat-moisture treated). This would then explain the observed order of increase in absorbance [defatted (Fig. 4.11b) $>$ native (Fig. $4.10 \mathrm{~b}$ ) > heat-moisture treated (Fig. $4.12 \mathrm{~b}$ )] between the $1^{\text {st }}$ and $35^{\text {th }}$ day of storage.

4.7.1.5 Turbidity development and scanning electron micrographs of alkaline treated native, defatted and heat-moisture treated starches during storage

In all starches, the extent of absorbance increase during the first $24 \mathrm{~h}$ of storage was less pronounced after alkaline treatment (Fig. 4.10a, 4.11a, 4.12a). For instance, during the above time period absorbance increased by only 0.34 . 0.55 and 0.77 , respectively, in alkali treated native (Fig. 4.10a), defatted (Fig. 4.11a) and heat-moisture treated (Fig. 4.12a) starches. The corresponding values for untreated starches were 2.48 (native), 1.35 (defatted) and 0.81 (heatmoisture treated), respectively. However, the extent of absorbance increase between the $1^{\text {st }}$ and $35^{\text {th }}$ day of storage was more pronounced after alkaline treatment. Scanning electron micrographs of fresh (Fig. 4.14a) and stored ( $24 \mathrm{~h}$ at $4^{\circ} \mathrm{C}$ [Fig. $\left.4.14 \mathrm{C}\right]$ ) pastes of alkali treated native starches were similar. In fresh pastes of alkali treated defatted (Fig. 4.15a) and heat-moisture treated (Fig. 4.16a) starches, granule remnants were scattered throughout the matrix. The size of these remnants was larger in heat-moisture treated starch (Fig. 4.16a). 
However, in stored $\left(24 \mathrm{~h}\right.$ at $\left.4^{\circ} \mathrm{C}\right)$ pastes, granule remnants of both alkali treated defatted (Fig. 4.15c,d) and heat-moisture treated (Fig. 4.16c,d) starches were enmeshed in the amylose exudate. The above results have shown that the alkaline conditions used during hydroxypropylation influence starch chain aggregation during storage. In native starch, the magnitude of the increase in absorbance during $24 \mathrm{~h}$ of storage (at $4^{\circ} \mathrm{C}$ ) decreased drastically (Fig. $4.10 \mathrm{a}$ ) after alkaline treatment. However, in stored pastes of alkali treated defatted (Fig. 4.15c,d) and heat-moisture treated (Fig. 4.16c,d) starches, starch chain aggregation was hindered by aggregated granule remnants (larger in heatmoisture treated). This would explain, differences in absorbance increase (during the $24 \mathrm{~h}$ storage period at $4^{\circ} \mathrm{C}$ ) between untreated and alkali treated starches [native (Fig. 4.10a) > defatted (Fig. 4.11a) $>$ heat-moisture treated (Fig. 4.12a)].

4.7.1.6 Turbidity development and scanning electron microscopy of hydroxypropylated native, defatted and heat-moisture treated starch pastes during storage

In hydroxypropylated native starches, absorbance increased with storage time, reaching a maximum value (after 3 days) of 0.28 and 0.24 , respectively, at MS 0.11 and 0.18 . Thereafter, absorbance decreased gradually reaching a value of 0.20 and 0.16 , respectively (after 35 days), at MS 0.11 and 0.18 (Fig. 4.10b). However, at MS 0.25 , absorbance remained constant $(\sim 0.15)$ until the 
$20^{\text {th }}$ day (Fig. 4.10b). Thereafter, absorbance decreased gradually reaching a value of 0.09 at the end of the storage period $\left(35^{\text {th }}\right.$ day). In hydroxypropylated defatted starch, absorbance continued to increase with storage time reaching a maximum value of 0.89 (MS 0.10), 0.77 (MS 0.17 ) and 0.47 (MS 0.20), respectively, after 5 days, 9 days and 1 day (Fig. 4.11b). Thereafter, absorption remained constant until $15^{\circ}$ day at MS 0.10 and 0.17 , and then decreased steeply reaching a value of 0.51 and 0.49 , respectively (after 35 days). at MS 0.10 and 0.17 (Fig. 4.11b). However, at MS 0.20 absorption continued to decrease gradually beyond day 1, reaching a value of 0.32 after 35 days (Fig. 4.11b).

In hydroxypropylated heat-moisture treated starch, absorption continued to increase throughout the storage period at MS 0.11 and 0.20 (Fig. 4.12a,b). The extent of this increase was more pronounced at MS 0.11. However, at MS 0.28 absorption increased only until the $18^{\text {th }} \mathrm{h}$ of storage (Fig. 4.12a). Thereafter, it remained unchanged throughout the storage period (Fig. 4.12a,b).

Scanning electron micrographs of stored (1 day) hydroxypropylated native (MS 0.11), defatted (MS 0.10) and heat-moisture treated (MS $(0.11)$ starches are presented in Fig. 4.17. In hydroxypropylated stored native starch paste (MS 0.11 ), the leached amylose chains and granule remnants were fused together to form a compactly packed structure (Fig. 4.17b). The morphology of the paste was nearly similar to that of stored alkali treated native starch (Fig 4.14c). Granule remnants, leached amylose and amylopectin chains of stored 
hydroxypropylated defatted starch (MS 0.10) were loosely packed in the matrix (Fig. 4.17d). Furthermore, the morphology of this paste was totally different from that of stored alkali treated defatted starch (Fig. 4.15c,d). In stored hydroxypropylated heat-moisture treated starch (MS 0.11), granule remnants, leached amylose and amylopectin chains were more loosely packed (Figs. 4.17 g,h) than in stored pastes of hydroxypropylated (MS 0.10) defatted starch (Fig. 4.17c,d).

The results on hydroxypropylation indicate that changes in absorbance during the entire storage period ( 35 days) is influenced by the interplay of 3 factors : 1) steric effects imposed by bulky hydroxypropyl groups (this decreases the magnitude and rate of increase in absorbance by hindering starch chain alignment during retrogradation); 2) presence of small granule remnants which gradually aggregate during storage (this would increase the intensity of scattered light); and 3) settling of large aggregated granule remnants below the path of the spectrophotometric beam (this would increase light transmittance).

In hydroxypropylated (at MS 0.11 and 0.18 ) native starch, the gradual increase in absorbance (Fig. $4.10 \mathrm{a}, \mathrm{b}$ ) during the first 3 days of storage is mainly due to slow aggregation of starch chains. The gradual decrease in absorbance beyond day 3 (Fig. $4.10 \mathrm{~b}$ ) at these MS levels, is mainly influenced by factor 2 . At MS 0.25 , absorbance remained unchanged until day 20 due to factor 1 (which prevents chain aggregation). The decrease in absorbance beyond day 20 at MS 0.25 (Fig. 4.10b) is mainly influenced by factor 2 . 
In defatted starch, the steep decrease (Fig. 4.11b) in absorbance (after the $15^{\text {th }}$ day) at MS 0.10 and 0.17 is mainly due to factor 3 . This decrease does not occur at MS 0.20 since granule remnants are probably disrupted [ due to the large increase in granular swelling that occur at this MS level (Table 4.12)]. Consequently, at MS 0.20 the changes in absorbance during storage are mainly influenced by starch chain aggregation.

As described earlier, granule crystallinity is destroyed on heat-moisture treatment. Consequently, heat-moisture treated granules would be more susceptible than defatted granules to disruption by the bulky hydroxypropyl groups. This would explain the absence of large granule remnants (Fig. 4.17e,f) after hydroxypropylation (MS 0.11). The extent of increase in absorbance during storage (35 days) of heat-moisture treated starches at the different MS levels (MS $0.11>$ MS $0.20>$ MS 0.28 ) is due to the interplay of factors 1 and 2. The results indicate that all granule remnants are not disrupted by hydroxypropyl groups at MS 0.11 (Fig. 4.17e,f). The undisrupted remnants probably aggregate slowly during storage. This would then explain the steep increase in absorption after $7^{\text {th }}$ day of storage at MS 0.11 . However, at MS 0.20 and 0.25 the extent of absorption increase is mainly influenced by factor 1 . 


\subsubsection{Enzyme digestibility}

4.7.2.1 Enzyme digestibility of freshly gelatinized and stored pastes of native, defatted and heat-moisture treated starches

Table 4.21 shows the enzyme digestibility of gelatinized and stored native, defatted and heat-moisture treated starch pastes. Freshly gelatinized pastes of native, defatted and heat-moisture treated starches were hydrolyzed nearly to the same extent by porcine pancreatic $\alpha$-amylase. Storage of gels at $4^{\circ} \mathrm{C}$ for $24 \mathrm{~h}$ decreased hydrolysis of native, defatted and heat-moisture treated starch gels by 5.6, 2.1 and 1.6\%, respectively (Table 4.21). However, storage beyond $24 \mathrm{~h}$ caused marginal decrease in hydrolysis in the above starches (Table 4.21).

Colonna et al. (1992) have shown that $\alpha$-amylase hydrolysis occurs in three successive steps : diffusion of $\alpha$-amylase towards its substrate, absorption of the enzyme on the substrate and the catalytic event. Many researchers have shown that enzyme susceptibility of a starch gel decreases with an increase in retrogradation (Ring et al., 1988; Eerlingen et al., 1994; Wang et al., 1995; Cui \& Oates, 1997). The following factors have been shown to be responsible for the above observations : 1) an increase in the entanglement of the molecules in the gel network; 2) an increase in molecular order in the short range (double-helix formation); and 3) an increase in the long range (crystallite formation) order. Wang et al. (1995) and Cui \& Oates (1997) have shown with sago starch gels, that the decrease in enzyme susceptibility during the first $6 \mathrm{~h}$ of storage at $5^{\circ} \mathrm{C}$. is mainly due to amylose retrogradation. 
Table $4.21 \alpha$-amylase hydrolysis of freshly gelatinized and stored pastes of native, defatted and heat-moisture treated starches

\begin{tabular}{lccc}
\hline $\begin{array}{l}\text { Starch source and } \\
\text { treatment }\end{array}$ & \multicolumn{3}{c}{$\begin{array}{c}\text { Hydrolysis (\%) } \\
\text { Storage time (days) }\end{array}$} \\
\hline Native & $77.2 \pm 0.8^{\mathrm{a}}$ & $71.6 \pm 0.7^{\mathrm{b}}$ & $69.0 \pm 1.1^{\mathrm{b}}$ \\
Heat-moisture treated $^{5}$ & $75.3 \pm 0.9^{\mathrm{a}}$ & $73.7 \pm 1.0^{\mathrm{ab}}$ & $71.2 \pm 0.5^{\mathrm{b}}$ \\
Defatted $^{6}$ & $76.2 \pm 1.0^{\mathrm{a}}$ & $74.1 \pm 0.9^{\mathrm{a}}$ & $74.1 \pm 0.4^{\mathrm{a}}$ \\
\hline
\end{tabular}

Hydrolysis for $3 \mathrm{~h}$ at $37^{\circ} \mathrm{C}$.

2Freshly gelatinized.

'Storage at $4^{\circ} \mathrm{C}$ for $24 \mathrm{~h}$.

'Storage at $4^{\circ} \mathrm{C}$ for $24 \mathrm{~h}$ and at $40^{\circ} \mathrm{C}$ for 14 days .

Heat-moisture treatment $\left(16 \mathrm{~h} / 100^{\circ} \mathrm{C} / 30 \%\right.$ moisture $)$.

Defatted with $75 \%$ propanol for $7 \mathrm{~h}$.

${ }^{r b}$ Means within a row with different superscripts are significantly different $(p \leq 0.05)$. 
When an aqueous starch suspension is heated above the gelatinization temperature, swelling is accompanied by the solubilization of amylose. Even on heating to $100^{\circ} \mathrm{C}$, there is little evidence for the release of substantial quantities of amylopectin (Ring et al.,1987; Morris, 1990). Thus, heating results in a medium composed of gelatinized swollen granules with an apparent amylopectin skeleton embedded in a hot amylose solution. As shown before, the extent of amylose leaching during gelatinization $\left(a 0^{\circ} \mathrm{C}\right.$ ) of native, defatted and heatmoisture treated potato starches were $22.7,13.4$ and $8.3 \%$, respectively (Table 4.4). Leached amylose chains would aggregate faster than those within gelatinized granules (due to steric effects imposed by branched amylopectin chains). Furthermore, aggregation between leached amylose chains would be much faster and stronger in pastes of gelatinized native starch (due to more leached amylose) than in the other starches. Thus, the observed decrease in enzyme hydrolysis during the first $24 \mathrm{~h}$ of storage (Table 4.21) [native > defatted $>$ heat-moisture treated] reflects mainly the rate and extent of aggregation between leached amylose chains.

4.7.2.2 Enzyme hydrolysis in freshly gelatinized and stored pastes of alkali treated native, defatted and heat-moisture treated starches

Enzyme digestibility of alkali ttreated starch pastes before and after storage is shown in Table 4.22. Alkaline treatment increased the extent of hydrolysis of freshly gelatinized pastes of native, defatted and heat-moisture 
Table 4.22 a-amylase hydrolysis of freshly gelatinized and stored pastes of alkali treated native, defatted and heat-moisture treated starches

\begin{tabular}{lccc}
\hline $\begin{array}{l}\text { Starch source and } \\
\text { treatment }\end{array}$ & \multicolumn{3}{c}{$\begin{array}{c}\text { Hydrolysis (\%) } \\
\text { Storage time (days) }\end{array}$} \\
\hline Native & $77.2 \pm 0.8^{\mathrm{a}}$ & $71.6 \pm 0.7^{\mathrm{b}}$ & $69.0 \pm 1.1^{\mathrm{b}}$ \\
Alkali treated $^{\mathrm{s}}$ & $93.4 \pm 1.2^{\mathrm{a}}$ & $87.0 \pm 0.4^{\mathrm{b}}$ & $85.5 \pm 1.0^{\mathrm{b}}$ \\
& & & \\
Heat-moisture treated $^{6}$ & $75.3 \pm 0.9^{\mathrm{a}}$ & $73.7 \pm 1.0^{\mathrm{ab}}$ & $71.2 \pm 0.5^{\mathrm{b}}$ \\
Alkali treated $^{5}$ & $81.6 \pm .0 .8^{\mathrm{a}}$ & $76.2 \pm 0.9^{\mathrm{b}}$ & $74.1 \pm 1.0^{\mathrm{b}}$ \\
& & & $74.1 \pm 0.4^{\mathrm{a}}$ \\
Defatted $^{7}$ & $76.2 \pm 1.0^{\mathrm{a}}$ & $74.1 \pm 0.9^{\mathrm{a}}$ & $78.3 \pm 0.4^{\mathrm{c}}$ \\
Alkali treated $^{5}$ & $83.5 \pm 0.9^{\mathrm{a}}$ & $80.1 \pm 1.2^{\mathrm{b}}$ & 7 \\
\hline
\end{tabular}

Thydrolysis for $3 \mathrm{~h}$ at $37^{\circ} \mathrm{C}$.

${ }^{2}$ Freshly gelatinized.

${ }^{3}$ Storage at $4^{\circ} \mathrm{C}$ for $24 \mathrm{~h}$.

${ }^{4}$ Storage at $4^{\circ} \mathrm{C}$ for $24 \mathrm{~h}$ and at $40^{\circ} \mathrm{C}$ for 14 days .

${ }^{5} \mathrm{P}$ arent starch treated with $\mathrm{NaOH}$ and $\mathrm{Na}_{2} \mathrm{SO}_{4}$ at $40^{\circ} \mathrm{C}$.

${ }^{6}$ Heat-moisture treatment $\left(16 \mathrm{~h} / 100^{\circ} \mathrm{C} / 30 \%\right.$ moisture).

7 Defatted with $75 \%$ propanol for $7 \mathrm{~h}$.

${ }^{2-c}$ Means within a row with different superscripts are significantly different ( $p \leq 0.05$ ). 
treated starches by $16.0,7.3$ and $6.0 \%$, respectively (Table 4.22 ). The increased hydrolysis on alkaline treatment can be attributed to increased accessibility of $\alpha$ amylase into the amorphous regions of the granule. This is made possible by repulsion between adjacent amylose chains (carrying negatively charged oxygens) which hinder rapid aggregation of amylose chains immediately after gelatinization. This increase is higher in alkali treated native starch due to more extensive amylose leaching. Storage of alkali treated starches at $4^{\circ} \mathrm{C}$ for $24 \mathrm{~h}$ decreased hydrolysis in native, defatted and heat-moisture treated starches by 4.6, 3.4 and $4.9 \%$, respectively (Table 4.22). In all starches, hydrolysis did not change significantly when storage was continued beyond $24 \mathrm{~h}$ (Table 4.22).

\subsubsection{Enzyme hydrolysis of freshly gelatinized and stored pastes of hydroxypropyl native, defatted and heat-moisture treated starches}

Enzyme hydrolysis results of hydroxypropylated native, defatted and heatmoisture treated starch pastes before and after storage are given in Table 4.23. In all starches, hydrolysis decreased on hydroxypropylation (Table 4.23). The extent of hydrolysis of freshly gelatinized native starch decreased by 11.2, 15.6 and $21.5 \%$, respectively, at MS $0.11,0.18$ and 0.25 (Table 4.23). The corresponding values for defatted starch were 14.0, 29.4 and $30.2 \%$, respectively, at MS $0.10,0.17$ and 0.20 (Table 4.23). For heat-moisture treated starch, these values were $5.5,26.3$ and $38.4 \%$, respectively, at MS $0.11,0.20$ and 0.28 (Table 4.23). However, hydrolysis decreased only marginally on 
Table 4.23 a-amylase hydrolysis of freshly gelatinized and stored pastes of hydroxypropyl native, defatted and heat-moisture treated starches

\begin{tabular}{|c|c|c|c|c|}
\hline \multirow[t]{2}{*}{$\begin{array}{l}\text { Starch source and } \\
\text { treatment }\end{array}$} & \multirow[t]{2}{*}{$\begin{array}{c}\text { Molar } \\
\text { substitution }\end{array}$} & \multicolumn{3}{|c|}{$\begin{array}{c}\text { Hydrolysis (\%) } \\
\text { Storage time (days) }\end{array}$} \\
\hline & & $0^{2}$ & $1^{3}$ & $15^{4}$ \\
\hline \multirow[t]{4}{*}{ Native } & $0.00^{5}$ & $93.4 \pm 1.2^{a}$ & $87.0 \pm 0.4^{6}$ & $85.5 \pm 1.0^{b}$ \\
\hline & 0.11 & $66.0 \pm 1.0^{a}$ & $64.2 \pm 1.0^{2 b}$ & $63.0 \pm 0.6^{b}$ \\
\hline & 0.18 & $61.6 \pm 0.7^{a}$ & $60.4 \pm 1.0^{a b}$ & $59.1 \pm 0.8^{b}$ \\
\hline & 0.25 & $55.7 \pm 0.3^{a}$ & $55.5 \pm 0.9^{a}$ & $55.0 \pm 0.7^{a}$ \\
\hline \multirow[t]{4}{*}{ Heat-moisture treated ${ }^{6}$} & $0.00^{5}$ & $81.6 \pm .0 .8^{a}$ & $76.2 \pm 0.9^{b}$ & $74.1 \pm 1.0^{\circ}$ \\
\hline & 0.11 & $69.8 \pm 0.7^{a}$ & $68.0 \pm 0.6^{\mathrm{ab}}$ & $67.4 \pm 0.9^{\circ}$ \\
\hline & 0.20 & $49.0 \pm 1.1^{2}$ & $47.8 \pm 0.9^{2 b}$ & $46.4 \pm 0.5^{\circ}$ \\
\hline & 0.28 & $36.9 \pm 0.6^{\pi}$ & $36.1 \pm 0.4^{a}$ & $36.5 \pm 0.9^{a}$ \\
\hline \multirow[t]{4}{*}{ Defatted $^{7}$} & $0.00^{5}$ & $83.5 \pm 0.9^{a}$ & $80.1 \pm 1.2^{b}$ & $78.3 \pm 0.4^{c}$ \\
\hline & 0.10 & $62.2 \pm 0.7^{a}$ & $61.7 \pm 0.8^{a}$ & $59.3 \pm 1.0^{b}$ \\
\hline & 0.17 & $46.8 \pm 0.8^{a}$ & $45.0 \pm 1.0^{a}$ & $44.1 \pm 0.7^{\circ}$ \\
\hline & 0.20 & $46.4 \pm 0.9^{a}$ & $45.6 \pm 0.8^{a}$ & $45.4 \pm 1.1^{\mathrm{a}}$ \\
\hline
\end{tabular}

\footnotetext{
Thydrolysis for $3 \mathrm{~h}$ at $37^{\circ} \mathrm{C}$.

${ }^{2}$ Freshly gelatinized.

${ }^{3}$ Storage at $4^{\circ} \mathrm{C}$ for $24 \mathrm{~h}$.

${ }^{4}$ Storage at $4^{\circ} \mathrm{C}$ for $24 \mathrm{~h}$ and at $40^{\circ} \mathrm{C}$ for 14 days .

${ }^{5}$ Parent starch treated with $\mathrm{NaOH}$ and $\mathrm{Na}_{2} \mathrm{SO}_{4}$ at $40^{\circ} \mathrm{C}$ (alkali treated).

${ }^{6}$ Heat-moisture treatment $\left(16 \mathrm{~h} / 100^{\circ} \mathrm{C} / 30 \%\right.$ moisture).

${ }^{7}$ Defatted with $75 \%$ propanol for $7 \mathrm{~h}$.

${ }^{-c}$ Means within a row with different superscripts are significantly different $(p \leq 0.05)$.
} 
storage of hydroxypropylated starches (Table 4.23). For instance, at a nearly comparable MS level $(\sim 0.10-0.11)$, the decrease in hydrolysis after a 15 day storage period was only $3.0,2.9$ and $3.4 \%$, respectively, in native (MS 0.11), defatted (MS 0.10) and heat-moisture treated (MS 0.11) starches [Table 4.23].

The decrease in hydrolysis on hydroxypropylation is in agreement with Leegwater \& Luten (1971), Wootton \& Chaudhry (1981) and Hoover et al. (1988). A study of the catalytic groups of $\alpha$-amylase has shown that carboxylate anion acts as the nucleophile and imidazolium cation as the electrophile (Robyt, 1984). It is possible that the decrease in hydrolysis with increase in MS may be due to bulky hydroxypropyl groups on C-2 sterically hindering the action of the catalytic carboxylate ion on the glycosidic bond. The marginal change in hydrolysis on storage (Table 4.23) suggests that hydroxypropylation is very effective in hindering starch chain aggregation within the amorphous domain of the granule.

\subsubsection{X-ray diffraction}

4.7.3.1 X-ray diffraction of fresh and stored gels of native, defatted and heat-moisture treated starches

The X-ray diffraction patterns of freshly gelatinized and stored (1 day at $4^{\circ} \mathrm{C}$ and for 29 days at $40^{\circ} \mathrm{C}$ ) pastes of native, defatted and heat-moisture treated starches are presented in Figs. 4.18, 4.19 and 4.20. No significant peak could be distinguished in freshly gelatinized native (Fig. 4.18a), defatted (Fig. 
Fig. 4.18 X-ray diffraction patterns of freshly gelatinized and stored pastes $(24 \mathrm{~h}$ at $4^{\circ} \mathrm{C}$ and then at $40^{\circ} \mathrm{C}$ for 29 days) of untreated, alkali treated and hydroxypropylated (MS 0.18 ) native potato starches: (A) freshly gelatinized untreated native starch, (B) untreated native starch after 30 days storage, (C) freshly gelatinized alkali treated native starch, (D) alkali treated native starch after 30 days storage, $(E)$ freshly gelatinized hydroxypropylated (MS 0.18) native starch, (F) hydroxypropylated (MS 0.18) native starch after 30 days storage. 


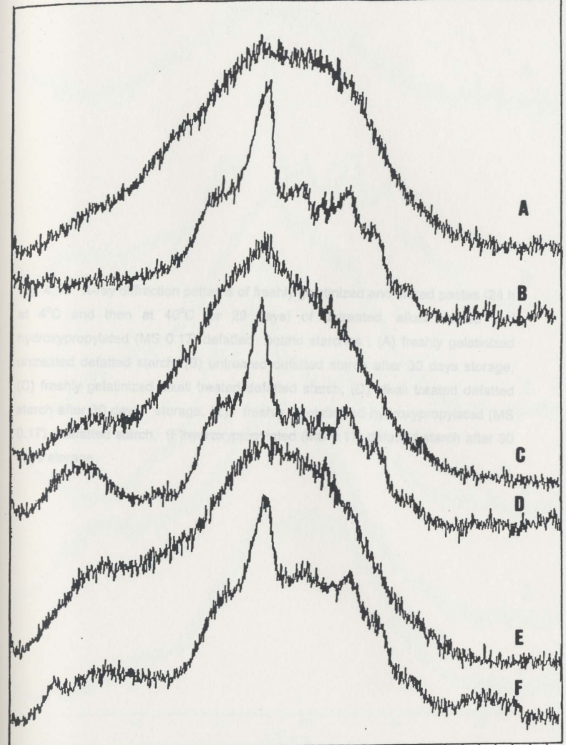

4 
Fig. 4.19 $\mathrm{X}$-ray diffraction patterns of freshly gelatinized and stored pastes ( $24 \mathrm{~h}$ at $4^{\circ} \mathrm{C}$ and then at $40^{\circ} \mathrm{C}$ for 29 days) of untreated, alkali treated and hydroxypropylated (MS 0.17 ) defatted potato starches : (A) freshly gelatinized untreated defatted starch, (B) untreated defatted starch after 30 days storage, (C) freshly gelatinized alkali treated defatted starch, (D) alkali treated defatted starch after $\mathbf{3 0}$ days storage, (E) freshly gelatinized hydroxypropylated (MS 0.17 ) defatted starch, (F)hydroxypropylated (MS 0.17) defatted starch after 30 days storage. 
Fig. 4.20 $\mathrm{X}$-ray diffraction patterns of freshly gelatinized and stored pastes ( $24 \mathrm{~h}$ at $4^{\circ} \mathrm{C}$ and then at $40^{\circ} \mathrm{C}$ for 29 days) of untreated, alkali treated and hydroxypropylated (MS 0.20) heat-moisture treated potato starches : (A) freshly gelatinized untreated heat-moisture treated starch, $(B)$ untreated heat-moisture treated starch after $\mathbf{3 0}$ days storage, (C) freshly gelatinized alkali treated heatmoisture treated starch, (D) alkali treated heat-moisture treated starch after 30 days storage, (E) freshly gelatinized hydroxypropylated (MS 0.20 ) heat-moisture treated starch, (F) hydroxypropylated (MS 0.20) heat-moisture treated starch after 30 days storage. 


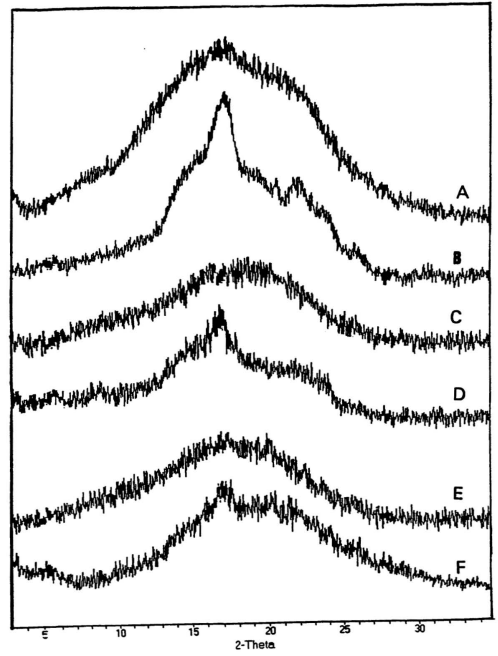


4.19a) and heat-moisture treated starches (Fig. 4.20a). The 'B' type $X$-ray pattern which is typical of retrograded starch (d spacings at 16, 5.2, 3.9 and 3.7) was evident (in all three starches) at the end of the storage period (Figs. 4.18b. $4.19 \mathrm{~b}, 4.20 \mathrm{~b})$. The intensity of the spacing centred at $5.2 \mathrm{~A}$ followed the order : defatted > native > heat-moisture treated (Table 4.24). As described earlier, the distance separating the outer branches of amylopectin chains in gelatinized starches followed the order : heat-moisture treated $>$ native > defatted. Thus, due to closer proximity of the outer branches in gelatinized defatted starch, double helical formation and packing of double helices during storage would be much easier and more ordered in defatted than in other starches. This would then account for the stronger $\mathrm{X}$-ray diffraction pattern of retrograded defatted starch (Fig. 4.19b). It has been shown that the ' $\mathrm{B}$ ' pattern originates from hexagonal packing of double helices during retrogradation of both amylose (Gidley, 1989) and amylopectin (Zobel, 1988b). As shown in Fig. 4.13, granule remnants are present in gelatinized pastes of defatted (Fig. 4.13c) and heatmoisture treated starches (Fig. 4.13e). The size of these remnants being greater in the latter. The presence of granule remnants could hinder aggregation and packing of double helices of both amylose and amylopectin. Thus, the recorded intensities are probably influenced by the interplay of two factors : 1) degree of proximity of the outer branches of amylopectin chains to each other in gelatinized pastes; and 2) size of the granule remnants in the gelatinized paste. 
Table 4.24 X-ray diffraction intensities (at 5.2A) of stored ${ }^{1}$ potato starch gels Starch source \& treatment Moisture (\%) Intensity $\left(\mathrm{cps}^{2}\right)$ at 5.2A

\begin{tabular}{|c|c|c|}
\hline Native & 9.6 & 1535 \\
\hline Native (alkali treated) ${ }^{3}$ & 9.5 & 1496 \\
\hline Native $\left(\mathrm{MS}^{4} \mathrm{0.18}\right)$ & 9.6 & 990 \\
\hline Heat-moisture treated ${ }^{5}$ & 9.5 & 1225 \\
\hline Heat-moisture treated (alkali treated) ${ }^{3}$ & 9.6 & 232 \\
\hline Heat-moisture treated $\left(\mathrm{MS}^{4} 0.20\right)$ & 9.5 & 194 \\
\hline Defatted $^{6}$ & 9.4 & 1895 \\
\hline Defatted(alkali treated) ${ }^{3}$ & 9.5 & 1383 \\
\hline Defatted $\left(\mathrm{MS}^{4} 0.17\right)$ & 9.5 & 715 \\
\hline
\end{tabular}

${ }^{1}$ One day at $4^{\circ} \mathrm{C}$ and then at $40^{\circ} \mathrm{C}$ for 29 days.

${ }^{2}$ Counts per second.

${ }^{3}$ Parent starch treated with $\mathrm{NaOH}$ and $\mathrm{Na}_{2} \mathrm{SO}_{4}$ at $40^{\circ} \mathrm{C}$.

${ }^{4}$ Molar substitution.

${ }^{5}$ Heat-moisture treatment $\left(16 \mathrm{~h} / 100^{\circ} \mathrm{C} / 30 \%\right.$ moisture).

${ }^{6}$ Defatted with $75 \%$ propanol for $7 \mathrm{~h}$. 
4.7.3.2 X-ray diffraction of fresh and stored gels of alkali treated native, defatted and heat-moisture treated starches

No significant peak could be distinguished in freshly gelatinized pastes of native (Fig. 4.18c), defatted (Fig. 4.19c) and heat-moisture treated (Fig. 4.20c) starches. In all alkali treated starches, the X-ray diffraction pattern and the intensity of the $5.2 \mathrm{~A}$ peak after 30 days of storage was weaker than in their untreated counterparts (Table 4.24). The extent of this intensity reduction followed the order : heat-moisture treated $>$ defatted $>$ native (Table 4.24). This decrease can be attributed to the size of granule remnants (heat-moisture treated > defatted > native) in the alkali treated pastes, which would hinder the formation, aggregation and packing of double helices in a crystalline array.

4.7.3.3 X-ray diffraction of fresh and stored gels of hydroxypropyl native, defatted and heat-moisture treated starches

No significant peaks could be distinguished in freshly gelatinized pastes of hydroxypropylated native (Fig. 4.18e), defatted (Fig. 4.19e) and heat-moisture treated (Fig. 4.20e) starches. The intensities at $5.2 \AA$ decreased in stored hydroxypropylated starch gels (Table 4.24). The extent of this decrease in native (MS 0.18), defatted (MS 0.17 ) and heat-moisture treated (MS 0.20) hydroxypropylated starches were $35.5,62.2$ and $84.2 \%$, respectively. This decrease is due to a decrease in chain aggregation resulting from the interplay of two factors : 1) steric effects imposed by hydroxypropyl groups on adjacent 
starch chains; and 2) aggregation of large granule remnants of hydroxypropylated defatted (MS 0.17) [Fig. 4.17d] and heat-moisture treated (MS $0.20)$ [Fig. 4.17g] starches.

\subsubsection{Differential scanning calorimetry (DSC)}

4.7.4.1 DSC parameters of native, defatted and heat-moisture treated starches after gelatinization and storage

In all starches, the retrogradation endotherm occurred only after 2 days of storage (Table 4.25). Retrogradation enthalpy $\left(\Delta \mathrm{H}_{R}\right)$ at the end of the storage period ( 7 days) was more pronounced in native $(6.7 \mathrm{~J} / \mathrm{g})$ than in defatted $(4.5 \mathrm{~J} / \mathrm{g})$ or heat-moisture treated $(4.8 \mathrm{~J} / \mathrm{g})$ starches. The transition temperatures $T_{0} . T_{p}$ and $T_{c}$ of the retrogradation endotherm of native starch were higher than those of defatted and heat-moisture treated starches. However, differences between $T_{0}, T_{p}$ and $T_{c}$ of defatted and heat-moisture treated starches were only marginal (Table 4.25), In all starches, $T_{0} . T_{p}$ and $T_{c}$ remained practically unchanged over the day 7 of storage. The storage of gels beyond 7 days produced endotherms which were too broad for accurate determination of transition temperatures and $\Delta \mathrm{H}_{\mathrm{R}}$.

Recrystallization of starch molecules occurs during gel storage. Reheating of an aged starch gel in a DSC produces an endothermic transition which is not present in the DSC scan of freshly gelatinized samples. Such a transition is 


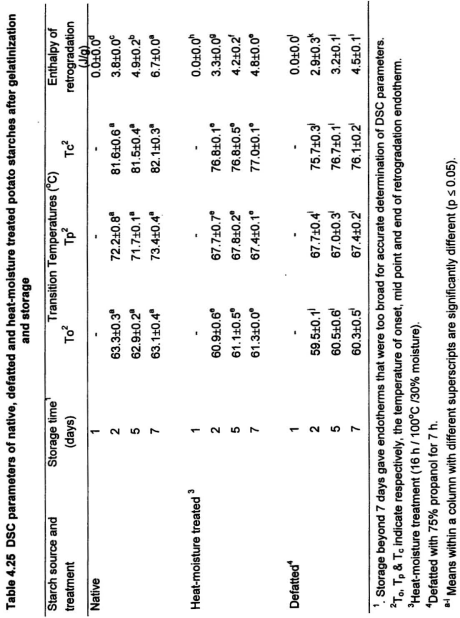


generally attributed to the melting of crystallized amylopectin. This was also proven by the thermo-reversible crystallization of amylose free (obtain by leaching) swollen starch granules (Miles et al., 1985b). The thermo-reversibility is attributed to the involment of outer branches of amylopectin which gives rise to low melting crystals (Morris, 1990). The enthalpy of retrogradation is generally considered to correspond to order-disorder transitions of crystallites (i.e. double helices present in extended ordered arrays) and regions of lesser crystalline order. Numerous researchers (Ring et al., 1987; Russel, 1987; Kalichevsky et al., 1990; Ward et al., 1994) have observed retrogradation endotherm at similar temperatures well below the temperature range for gelatinization. Recrystallization of the amylopectin branch chains in a less ordered manner than what existed for the native starch is an explanation for the observed amylopectin retrogradation endotherm at a temperature range below that for gelatinization (Ward et al., 1994).

Amylose crystals formed in starch gels contain longer helical sequences which melt at higher temperatures. Thus, the melting of amylose crystallites were not observed within the heating temperature range studied $\left(20-100^{\circ} \mathrm{C}\right)$ in DSC. The absence of retrogradation endotherm on day 1 suggests that amylose crystallization takes place mainly during the first $24 \mathrm{~h}$ of storage, after which amylopectin crystallization occurs at a slow rate, which was implied by the gradual increase in $\Delta H_{R}$. 
Hoover \& Vasanthan (1994a) have shown that during heat-moisture treatment crystallinity is disrupted within granules of potato starch. Thus, after gelatinization the degree of separation between the outer branches of adjacent amylopectin chain clusters would be more pronounced in heat-moisture treated than in native starch. Consequently, double helical formation (during storage) between adjacent amylopectin chains of gelatinized heat-moisture treated starch would be much slower and reduced in number than in native starch. This would explain the slower increase in $\Delta \mathrm{H}_{R}$ (during storage) and its decreased magnitude after heat-moisture treatment (Table 4.25). Vasanthan \& Hoover (1992a) have aiso shown that defatting increases granule crystallinity in potato starch. Thus, the number of ordered double helices that disrupt on gelatinization would be much lower in defatted than in native starch. Consequently, the number of new double helices that form during retrogradation would be much decreased after defatting. This would explain the slower increase in $\Delta \mathrm{H}_{R}$ (during storage) and its decreased magnitude after defatting (Table 4.25).

\subsubsection{DSC parameters of alkall treated native, defatted and heat-moisture treated starches after gelatinization and storage}

In all starches, retrogradation endotherm appeared on the day 2 of storage (Table 4.26). The $\Delta H_{R}$ of alkali treated starch gels increased with increase in storage time, whereas the transition temperatures remained unchanged during the time course of retrogradation. However, the magnitude 
Table 4.26 DSC parameters of alkall treated ${ }^{1}$ native, defatted and heat-molsture treated potato starches

\begin{tabular}{|c|c|c|c|c|c|}
\hline \multirow{2}{*}{$\begin{array}{l}\text { Starch source } \\
\text { and treatment }\end{array}$} & \multirow{2}{*}{$\begin{array}{l}\text { Storage time } \\
\text { (days) }\end{array}$} & \multicolumn{3}{|c|}{ Transition temperature $\left({ }^{\circ} \mathrm{C}\right)$} & \multirow{2}{*}{$\begin{array}{c}\text { Enthalpy of } \\
\text { retrogradation } \\
(\mathrm{J} / \mathrm{g})\end{array}$} \\
\hline & & $\mathrm{To}^{3}$ & $T p^{3}$ & $\mathrm{Tc}^{3}$ & \\
\hline \multirow[t]{4}{*}{ Native } & 1 & $\cdot$ & - & $\cdot$ & $0.0 \pm 0.0^{\mathrm{d}}$ \\
\hline & 2 & $62.1 \pm 0.4^{a}$ & $70.6 \pm 0.5^{\mathrm{a}}$ & $80.3 \pm 0.2^{\circ}$ & $3.7 \pm 0.1^{\circ}$ \\
\hline & 5 & $62.5 \pm 0.5^{a}$ & $70.4 \pm 0.3^{\mathrm{a}}$ & $80.2 \pm 0.7^{a}$ & $4.6 \pm 0.2^{b}$ \\
\hline & 7 & $62.5 \pm 0.4^{a}$ & $71.0 \pm 0.3^{\mathrm{a}}$ & $81.1 \pm 0.2^{b}$ & $6.3 \pm 0.0^{\mathrm{n}}$ \\
\hline \multirow[t]{4}{*}{ Heat-moisture treated ${ }^{4}$} & 1 & - & - & - & $0.0 \pm 0.0^{h}$ \\
\hline & 2 & $61.4 \pm 0.1^{t}$ & $70.6 \pm 0.5^{1}$ & $81.1 \pm 0.8^{\prime}$ & $4.3 \pm 0.1^{0}$ \\
\hline & 5 & $62.6 \pm 0.4^{\circ}$ & $71.6 \pm 0.8^{\mathrm{et}}$ & $81.5 \pm 0.5^{\prime}$ & $6.1 \pm 0.1^{\prime}$ \\
\hline & 7 & $62.1 \pm 0.3^{\circ}$ & $71.7 \pm 0.4^{\circ}$ & $82.9 \pm 0.2^{\circ}$ & $7.3 \pm 0.1^{\circ}$ \\
\hline \multirow[t]{4}{*}{ Defatted $^{5}$} & 1 & - & - & - & $0.0 \pm 0.0^{\prime}$ \\
\hline & 2 & $63.3 \pm 0.2^{\prime}$ & $73.0 \pm 0.0^{\prime}$ & $80.6 \pm 0.2^{1}$ & $2.0 \pm 0.1^{k}$ \\
\hline & 5 & $63.3 \pm 0.2^{1}$ & $72.4 \pm 0.3$ & $80.8 \pm 0.6^{1}$ & $3.2 \pm 0.1$ \\
\hline & 7 & $62.1 \pm 0.2^{\prime}$ & $72.2 \pm 0.9^{\| \prime}$ & $80.6 \pm 0.1^{\prime}$ & $4.5 \pm 0.2^{1}$ \\
\hline
\end{tabular}

'Parent starch treated with $\mathrm{NaOH}$ and $\mathrm{Na}_{2} \mathrm{SO}_{4}$ at $40^{\circ} \mathrm{C}$

${ }^{2}$ Storage beyond 7 days gave endotherms that were too broad for accurate determination of DSC parameters.

${ }^{3} T_{0}, T_{p} \& T_{c}$ indicate respectively, the temperature of onset, mid point and end of retrogradation endotherm.

${ }^{4}$ Heat-moisture treatment $\left(16 \mathrm{~h} / 100^{\circ} \mathrm{C} / 30 \%\right.$ moisture).

${ }^{5}$ Defatted with $75 \%$ propanol for $7 \mathrm{~h}$.

${ }^{2-1}$ Means within a column under the same treatment with different superscripts are significantly different $(p \leq 0.05)$. 
and the rate of increase in $\Delta \mathrm{H}_{\mathrm{R}}$ (during storage) decreased slightly after alkali treatment. The difference in magnitude of $\Delta \mathrm{H}_{\mathrm{R}}$ between untreated and alkali treated starches was more pronounced in defatted than in native and heatmoisture treated starches (Table 4.26). The results indicate that the number of double helices that form on storage is reduced after alkaline treatment. This can be attributed to repulsion between negatively charged oxygens on adjacent interacting starch chains.

\subsubsection{DSC parameters of hydroxypropylated native (MS 0.11), defatted (MS} $0.10)$ and heat-moisture treated (MS 0.11) starches after gelatinization and storage

In hydroxypropylated starches (Table 4.27), the retrogradation endotherm occurred only near the end (7 days) of the storage period. Hydroxypropylation decreased $\Delta \mathrm{H}_{R}$ in all starches (Table 4.27). At the same MS $(0.11)$, the extent of decrease in $\Delta \mathrm{H}_{\mathrm{R}}$ (Day 7) was more pronounced in native than in heat-moisture treated starch (Table 4.27). This decrease in $\Delta H_{R}$ suggests that hydroxypropyl groups within the bulk amorphous and intercrystalline amorphous regions hinder double helical formation by preventing proper alignment of the outer branches of the amylopectin chains during storage. The reduction in $\Delta \mathrm{H}_{R}$ on hydroxypropylation was more pronounced in native than in defatted starch (Table 4.27) due to two reasons : 1) the lower MS level of defatted starch (MS 


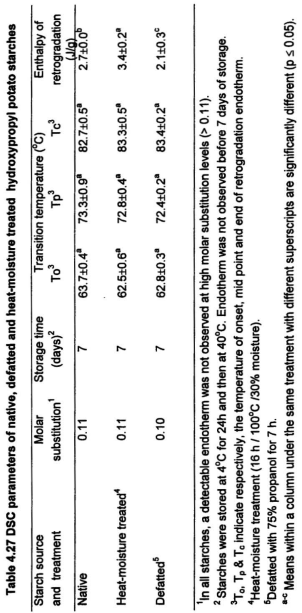


0.10); and 2) the presence of higher amounts of crystallites in the gelatinized defatted starch paste (defatting increases crystallinity) which partially negates the influence of hydroxypropyl groups on chain alignment.

Furthermore, the crystallites formed during storage of hydroxypropylated defatted and hydroxypropylated heat-moisture treated gels melted at a broader temperature range (hydroxypropylated defatted, Tc-To $=20.6^{\circ} \mathrm{C}$ : hydroxypropylated heat-moisture treated, Tc-To $=20.8^{\circ} \mathrm{C}$ [Table 4.27$]$ ) than did their untreated counterparts (defatted, Tc-To $=15.8^{\circ} \mathrm{C}$, heat-moisture treated, TC-To $=15.7^{\circ} \mathrm{C}$ [Table 4.25] ). This broadening is possibly due to the formation of loosely packed gels (as shown by the scanning electron micrographs Fig. $4.17 \mathrm{~d}, \mathrm{~h}$ ) which are composed of crystallites with different stabilities. However. broadening of crystallite melting temperature range was not observed in retrograded native starch gels after hydroxypropylation. 


\section{CHAPTER 5}

\section{SUMMARY AND CONCLUSIONS}

This study has shown that defatting causes clustering of the outer ' $\mathrm{A}$ ' chains of amylopectin, resulting in the formation of crystallites which are perfectly arrayed to diffract X-rays, whereas, crystallite disruption and/or reorientation of the double helices (within the crystalline array) occurs on heat-moisture treatment. Both defatting and heat-moisture treatment changed the $\mathrm{X}$-ray pattern from ' $B$ ' to ' $A+B$ '. However, the amount of ' $A$ ' unit cells was higher on heatmoisture treatment. Interactions between starch chains (AM-AM and AM-AP) occurred on defatting and heat-moisture treatment (heat-moisture treatment > defatting). Double helical content within the amorphous and crystalline domains decreased on heat-moisture treatment, but increased slightly on defatting. These structural changes decreased the SF, Brabender viscosity (at $95^{\circ} \mathrm{C}$ ) and $\mathrm{AML}$, and increased the thermal stability and gelatinization transition temperatures of both defatted and heat-moisture treated starches. However, $\Delta \mathrm{H}$ decreased on heat-moisture treatment, but increased on defatting .

The reagents $\left(\mathrm{NaOH}\right.$ and $\left.\mathrm{Na}_{2} \mathrm{SO}_{4}\right)$ used during hydroxypropylation altered crystallite orientation, disrupted double helices (within the amorphous regions). increased SF, and decreased Brabender viscosity (at $95^{\circ} \mathrm{C}$ ). The extent of the above changes followed the order : native > defatted > heat-moisture treated. However, starch chain interactions did not occur under these reaction conditions. 
The degree of accessibility of hydroxypropyl groups into the granule interior (heat-moisture treated > native > defatted) was found to be influenced by granule crystallinity (defatted $>$ native $>$ heat-moisture treated). X-ray diffraction patterns showed that hydroxypropylation occurs mainly in the amorphous regions.

The SF (at different temperatures) of hydroxypropylated (at different MS levels) native, defatted and heat-moisture treated starches was influenced by the interplay between the extent of hydrogen bond disruption within the amorphous regions (due to hydroxypropylation) and the increased interaction that occurs between starch chains during defatting and heat-moisture treatment. Hydroxypropylation decreased amylose leaching. The DSC results showed that hydroxypropylation decreased $T_{0}, T_{p}$ and $T_{c}$ (due to hydrogen bond disruption) and $\Delta \mathrm{H}$ (due to double helical disruption). The magnitude of this decrease was influenced by the MS level. The pasting temperatures of all three starches decreased with increased MS. Increase in the level of MS progressively increased Brabender viscosity $\left(95^{\circ} \mathrm{C}\right)$ for defatted starch. However, for native and heat-moisture treated starches, an increase in the level of MS beyond 0.18 and 0.20 , respectively, resulted in a decrease in Brabender viscosity $\left(95^{\circ} \mathrm{C}\right)$. The changes in pasting temperature and viscosity with increase in MS levels were attributed to the interplay between changes in SF and hydrogen bond disruption.

The enzyme digestibility study showed that the susceptibility of potato starch granules towards hydrolysis by porcine pancreatic $\alpha$-amylase increased 
progressively with the time of defatting (due to the release of entrapped amylose chains into the amorphous domains of granules). However, defatting beyond $9 \mathrm{~h}$, decreased hydrolysis (due to interactions between the outer branches of amylopectin chains and between the neighboring segments of the released amylose chains) to a level that was lower than that observed for native starch. Heat-moisture treatment was also found to increase granule susceptibility towards hydrolysis by $\alpha$-amylase. A steep increase in hydrolysis occurred during the first $8 \mathrm{~h}$ of heat-moisture treatment (due to crystallite disruption), followed by a gradual decrease (due to AM-AM and AM-AP interactions). However, at all time intervals, the level of hydrolysis in heat-moisture treated starch was higher than that of native starch.

The alkaline conditions used during hydroxypropylation were found to increase the susceptibility of native, defatted and heat-moisture treated starches towards hydrolysis by $\alpha$-amylase (native > defatted $>$ heat-moisture treated ). The extent of this increase was attributed to the interplay of 3 factors : a) disruption of double helices, b) ionization of the hydroxyl groups on adjacent starch chains, and c) an increase in granule solubility.

The susceptibility of native, defatted and heat-moisture treated starches towards hydrolysis by $\alpha$-amylase increased with MS levels (due to increased granular swelling). However, hydrolysis decreased (due to steric effects imposed by the bulky hydroxypropyl groups) at MS levels of 0.29 (native), 0.28 (heat- 
moisture treated) and 0.26 (defatted). The results showed that the structural changes that occur on defatting and heat-moisture treatment do not influence the mechanism by which hydroxypropyl groups influence the reactivity of the granule towards $\alpha$-amylase.

The retrogradation properties of native defatted and heat-moisture treated potato starches before and after hydroxypropylation were monitored using different techniques. The results showed that changes in turbidity during storage of native, defatted and heat-moisture treated starch pastes were influenced by the interplay of : 1) interaction between leached starch chains, 2) interaction between granule remnants and the leached exudate. However, in alkali treated native, defatted and heat-moisture treated starch pastes, turbidity changes on storage were influenced by aggregation of granule remnants. The extent followed the order : native > defatted >heat-moisture treatment. Hydroxypropylation decreased the rate and extent of increase in turbidity during storage of all three starches. The change in turbidity during storage of hydroxypropylated starch pastes was influenced by the interplay between : 1) steric effects imposed by hydroxypropyl groups on chain aggregation, 2) aggregation between small granule remnants, and 3) settling of large granule remnants beneath the path of the spectrophotometer beam. Reactivity of gelatinized pastes of native, defatted and heat-moisture treated starches towards porcine pancreatic $\alpha$-amylase decreased on storage. A similar trend was also observed after alkaline treatment. However, hydrolysis remained unchanged 
throughout the storage period in hydroxypropylated starches. Stored pastes of native, defatted and heat-moisture treated starches gave a ' $\mathrm{B}$ ' type $\mathrm{X}$-ray pattern. A similar pattern was also observed after alkaline treatment, and hydroxypropylation. However, the X-ray intensity of the peak at $5.2 \mathrm{~A}$ decreased after alkaline treatment and hydroxypropylation. The retrogradation endotherm (monitored by DSC) occurred after 2 days storage in native, defatted and heatmoisture treated starches. A similar trend was also observed after alkaline treatment. However, the retrogradation endotherm appeared only after 7 days in hydroxypropylated starches. The enthalpy of retrogradation in all starches decreased on alkaline treatment and hydroxypropylation. These results showed that, retrogradation properties of native, defatted and heat-moisture treated starches are indirectly influenced by the structural arrangement of starch chains within the amorphous and crystalline regions of the ungelatinized granule, which in turn, influence the extent of granule breakdown during gelatinization, and the interactions that occur between starch chains during gel storage. 


\subsection{IMPORTANCE OF THIS STUDY TO STARCH CHEMISTS, AND DIRECTIONS FOR FUTURE RESEARCH}

This research has provided additional information on the mechanism of starch chain rearrangements within the amorphous and crystalline domains of the potato starch granule under conditions of defatting and heat-moisture treatment. Conditions similar to heat-moisture treatment might be expected to occur during food processing when such conditions are met. Thus, this study would provide a deeper insight into the structure and physicochemical changes that occur during hydrothermal treatment of foods. This study has also shown that granule crystallinity changes on heat-moisture treatment. Since crystallinity influences starch functionality, heat-moisture treatment may be one way of modifying the poor functional properties of legume and certain tuber starches (cassava, yam). Research should thus be carried out to improve the functional properties of these starches by heat-moisture treatment (under different time / temperature / moisture conditions regimes).

The trend in the food industry is moving towards natural ingredients. For years, scientists have been researching for starches that have all of the properties of a modified starch but without the chemical treatment.

Presently, starches are modified by acetylation and hydroxypropylation to reduce retrogradation rates (high retrogradation rates lead to poor freeze thaw stability). This research has indicated that heat-moisture treatment may provide starches with superior freeze thaw stability (since retrogradation rates would be 
drastically reduced after heat-moisture treatment, due to decrease in amylose leaching and granular swelling). Thus, starch chemists need to tailor the retrogradation properties of heat-moisture treated starches (by different time I temperature / moisture conditions) to a level that is presently met by chemical modification.

The mode of interaction of sugars and lipids with starch components is still in dispute (Evans \& Haisman, 1982; Germani et al., 1983; Eliasson, 1992; Bello-perez \& Paredes-lopez, 1995). Furthermore, the mechanism by which sugars influence starch properties is still not clearly understood. This is because many of these studies have been conducted on native starches. However, if the above studies are conducted on native, defatted, heat-moisture treated and alkali treated starches, a deeper insight into the mechanism can be obtained.

This work has covered those areas in which the most exciting developments are likely to occur. It will provide the impetus for food scientists and food processors to look for physical means by which starch properties can be tailored to requirements whilst, avoiding any toxicological problems. 


\section{BIBLIOGRAPHY}

AACC. (1984). Approved methods of the American Association of Cereal Chemists, $8^{\text {th }}$ ed. American Association of Cereal Chemists, Minnesota.

Aguilera, J.M. \& Stanley, D.W. (1990). Microstructural principles of food processing and engineering. Elsevier Applied Science, New York, p. 80.

Alexander, R.J. (1992). Carbohydrates used as fat replacers. In Developments in Carbohydrate Chemistry. R.J. Alexander \& H.F. Zobel. (Eds.). American Association of Cereal Chemists, Minnesota, pp 343-370.

Alloncle, M., \& Doublier, J.L. (1991). Viscoelastic properties of maize starch / hydrocolloid pastes and gels. Food Hydrocolloids, 5 : 455-467.

Atkins, D.P. \& Kennedy, J.F. (1985). A comparison of the susceptibility of two commercial grades of wheat starch for enzymic hydrolysis and their resultant oligosaccharide product spectra. Starch, $37: 421-427$.

Banks, W. \& Greenwood, C.T. (1971). Amylose : a non-helical biopolymer in aqueous solution. Polymer, 12 : 141-145.

Banks, W. \& Greenwood, C.T. (1975). Starch and its components. Edinburgh university press. Edinburgh.

Bello-Perez, L.A. \& Paredes-Lopez, O. (1995). Effect of solutes on retrogradation of stored starches and amylopectins : A calorimetric study. Starch, $47: 83-86$.

Bello-Perez, L.A. \& Paredes-Lopez, L. (1996). Starch and amylopectin - effect of solutes on clarity of pastes. Starch, 48 : 205-207.

Biliaderis, C.G., Maurice, T.J. \& Vose, J.R. (1980). Starch gelatinization phenomena studied by differential scanning calorimetry. J. Food Sci., 45 : 1669-1680.

Biliaderis, C.G. (1982). Physical characteristics, enzyme digestibility, and structure of chemically modified smooth pea and waxy maize starches. $J$. Agric. Food Chem., 30:925-930.

Biliaderis, C.G., Page, C.M., Slade, L. \& Sirett, R.R. (1985). Thermal behavior of amylose-lipid complexes. Carbohydr. Polym., 5 : 367-389. 
Biliaderis, C.G., Page, M.C., Maurice, T.J. (1986a). On the multiple melting transitions of starch/monoglyceride systems. Food Chem., 22 : 279-295.

Biliaderis, C.G., Page, M.C., Maurice, T.J. \& Juliano, B.O. (1986b). Thermal characterization of rice starches. A polymeric approach to phase transitions of granular starch. J. Agric. Food Chem., $34: 6-14$.

Biliaderis, C.G., Page, M.C., Maurice, T.J. (1986c). Non equilibrium melting of amylose - V complexes. Carbohydr. Polym., 6 : 269-288.

Biliaderis, C.G \& Galloway, G. (1989). Crystallization behavior of amylose -V complexes : Structure-property relationships. Carbohydr. Res., 189 :31-48.

Biliaderis, C.G. (1990). Thermal analysis of food carbohydrates. In Thermal analysis of foods. V.R. Harwalkar, \& C.Y. Ma. (Eds.). Elsevier Science Publishers, Oxford, pp 168-220.

Biliaderis, C.G. \& Seneviratne, H.D. (1990). Solute effects on the thermal stability of glycerol monostearate amylose complex. Carbohydr. Res., 208 : 199-213.

Biliaderis, C.G. \& Zawistowski, J. (1990). Viscoelastic behavior of aging starch gels : effects of concentration, temperature, and starch hydrolysates on network properties. Cereal Chem., $67: 240-246$.

Biliaderis, C.G. (1991). The structure and interactions of starch with food constituents. Can. J. Physiol. Pharmacol., 69 : 60-78.

Biliaderis, C.G., Tonogai, J.R., Perez, C.M. \& Juliano, B.O. (1993). Thermophysical properties of milled rice starch as influenced by variety and parboiling method. Cereal Chem., $70: 512-516$.

Biliaderis, C.G. \& Prokopowich, D.J. (1994). Effect of polyhydroxy compounds on structure formation in waxy maize starch gels : a calorimetric study. Carbohydr. Polym., 23 : 193-202.

Biliaderis, C.G., Arvanitoyannis, I., Izydorczyk, M.S. \& Prokopowich, D.J. (1997). Effect of hydrocolloids on gelatinization and structure formation in concentrated waxy maize and wheat starch gels. Starch, $49: 278-283$.

Blanshard, J.M.V., Bates, D.R., Muhr, A.H., Worcester, D.L. \& Higgins, J.S. (1984). Small angle neutron scattering studies of starch granule structure. Carbohydr. Polym., $4: 427-442$. 
Blanshard, J.M.V (1987). Starch granule structure and function : a physicochemical approach. In Starch : Properties and Potential, T. Galliard. (Ed.). John Wiley \& sons, New York, pp 16-54.

Bligh, E.G. \& Dyer, W.J. (1959). A rapid method of total lipid extraction and purification. Can. J. Biochem. Physiol., 37 : 911-917.

Bohlin, L. \& Eliasson, A.C. (1986). Shear stress relaxation of native and modified potato starch gels. Starch, $38: 120-124$.

Bruner, R.L. (1964). Determination of reducing value. In Methods in Carbohydrate Chemistry. Vol 4. R.L. Whistler. (Ed.). Academic Press, New York, pp 67-71.

Butler, L.E., Christianson, D.D., Scheerens, J.C. \& Berry, J.W. (1986). Buffalo gourd root starch. Part iv. Properties of hydroxypropyl derivatives. Starch, 38 : 156-159.

Caims, P., Miles, M.J. \& Morris, V.J. (1991). Studies of the effect of the sugars ribose, xylose and fructose on the retrogradation of wheat starch geis by $X$ ray diffraction. Carbohydr. Polym., 16 : 355-365.

Cairns, P., Sun, L., Morris, V.J. \& Ring, S.G. (1995). Physicochemical studies using amylose as an in vitro model for resistant starch. J. Cereal Sci., 21 : 3747.

Cameron, R.E., Durrani, C.M. \& Donald, A.M. (1994). Gelation of amylopectin without long range order. Starch, $46: 285-287$.

Carlson, T.L.G., Larsson, D., Dinh-Nguyen, N. \& Krog, K. (1979). A study of the amylose-monoglyceride complex by Raman spectroscopy. Starch, $31: 222$ 224.

Chang, S.M. \& Liu, L.C. (1991). Retrogradation of rice starches studied by differential scanning calorimetry and influence of sugars, $\mathrm{NaCl}$ and lipids. $J$. Food Sci., 56 : 564-566.

Cheetham, N.W.H. \& Tao, L. (1997). Amylose conformational transitions in binary DMSO/water mixtures. Starch, $49: 407-415$.

Chrastil, J. (1987). Improved colorimetric determination of amylose in starches or flours. Carbohydr. Res., 159 : 154-158. 
Christianson, D.D., Hodge, J.E., Osborne, D. \& Detroy, R.E. (1981). Gelatinization of wheat starch as modified by xanthan gum, guar gum and cellulose gum. Cereal Chem., $58: 513-517$.

Ciacco, C.F. \& Fernandes, J.L.A. (1979). Effect of various ions on the kinetics of retrogradation of concentrated wheat starch gels. Starch, $31: 51-53$.

Clark, A.H., Gidley, M.J., Richardson, R.K. \& Ross-Murphy, S.B. (1989). Rheological studies of aqueous amylose gels : The effect of chain length and concentration on gel modulus. Macromolecules, 22 : 346-351.

Colonna, P., Buleon, A. \& Mercier, C. (1981). Pisum sativum and Vicia faba carbohydrates : structural studies. J. Food Sci., 46 : 88-93.

Colonna, P., Buleon, A. \& Mercier, C. (1987). Physically modified starches. In Starch : Properties and potential. T. Galliard. (Ed.). John Wiley \& Sons, New York, pp 79-114.

Colonna, P., Leloup, V. \& Buleon, A. (1992). Limiting factors of starch hydrolysis. Eur. J. Clinc. Nutr., 46 : 517-532.

Colquhoun, I.J., Parker, R., Ring, S.G., Sun, L. \& Tang, H.R. (1995). An NMR spectroscopic characterization of the enzyme-resistant residue from $\alpha$ amylolysis of an amylose gel. Carbohydr. Polym., 27 : 255-259.

Colwell, K.H., Axford, D.W.E., Chamberlain, N. \& Elton, G.A.H. (1969). Effect of storage temperature on the aging of concentrated wheat starch gels. J. Sci. Food Agric., 20 : 550-555.

Conde-Petit, B. \& Escher, F. (1994). Influence of starch-lipid complexation on the aging behavior of high concentration starch gels. Starch, 46 : 172-177.

Cone, J.W. \& Wolters, M.G.E. (1990). Some properties and degradability of isolated starch granules. Starch, $42: 298-301$.

Cooke, D. \& Gidley, M.J. (1992). Loss of crystalline and molecular order during starch gelatinization : origin of the enthalpy transition. Carbohydr. Res., 227 : 103-112.

Craig, S.A.S., Maningat, C.C., Seib, P.A. \& Hoseney, R.C. (1989), Starch paste clarity. Cereal Chem., 66 : 173-182. 
Cui, R. \& Oates, C.G. (1997). The effect of retrogradation on enzyme susceptibility of sago starch. Carbohydr. Polym., 32 : 65-72.

D'Appolonia, B.L. (1972). Effect of bread ingredients on starch gelatinization properties as measured in the amylograph. Cereal Chem., 49 : 532-543.

De Graaf, R.A., Lammers, G., Janssen, L.P.B.M. \& Beenackers, A.A.C.M. (1995). Quantitative analysis of chemically modified starches by ' $H$ NMR spectroscopy. Starch, $47: 469-475$.

Donald, A.M., Waigh, T.A., Jenkins, P.J., Gidley, M.J., Debet, M. \& Smith, A. (1997). Internal structure of starch granules revealed by scattering studies. In Starch : Structure and Functionality. P.J. Fraizier, P. Richmond, \& A.M. Donald. (Eds.). The Royal Society of Chemistry, Cambridge, pp 172-179.

Donovan, J.W. (1979). Phase transition of the starch-water system. Biopolymers, $18: 263-275$.

Donovan, J.W. \& Mapes, C.J. (1980). Multiple phase transitions of starches and naegeli amylodextrins. Starch, 32 : 190-193.

Donovan, J.W., Lorenz, K. \& Kulp, K. (1983). Differential scanning calorimetry of heat-moisture treated wheat and potato starches. Cereal Chem., $60: 381$ 387.

Dublier, J.L. \& Choplin, L. (1989). A rheological description of amylose gelation. Carbohydr. Res., $27: 361-364$.

Durrani, C.M. \& Donald, A.M. (1995). Physical characterization of amylopectin gels. Polymer gels \& Network, 3 : 1-27.

Eerlingen, R.C., Jacobs, H. \& Delcour, J.A. (1994). Enzyme resistant starch. V. Effect of retrogradation of waxy maize starch on enzyme susceptibility. Cereal Chem., $71: 351-355$.

Eidam, D., Kulicke, W.M., Kuhn, K. \& Stute, R. (1995). Formation of maize starch gels selectively regulated by the addition of hydrocolloids. Starch, 47 : 378-384.

Eliasson, A.C. (1985). Retrogradation of starch as measured by differential scanning calorimetry. In New approaches to research on Cereal Carbohydrates. R.D. Hill \& L. Munck. (Eds.). Elsevier Science Publishers, Amsterdam, pp 93-98. 
Eliasson, A.C. \& Krog, N. (1985). Physical properties of amylose-monoglyceride complexes. J. Cereal Sci., $3: 239-248$.

Eliasson, A.C., Finstad, H. \& Ljunger, G. (1988). A study of starch-lipid interactions for some native and modified maize starches. Starch, $40: 95-$ 100.

Eliasson, A.C. \& Ljunger, G. (1988). Interaction between amylopectin and lipid additives during retrogradation in a model system. J. Sci. Food Agric., 44 : 353-361.

Eliasson, A.C. (1992). A. Calorimetric investigation of the influence of sucrose on the gelatinization of starch. Carbohydr. Polym., 18 : 131-138.

Eliasson, A.C. \& Larsson, K. (1993). Physicochemical behavior of the components of wheat flour. In Cereals in Bread making. O.R. Fennema, M. Karel, G.W. Sanderson, S.R. Tannenbaum, P. Walstra \& J.R. Whitaker. (Eds.). Marcel Dekker Inc., New York, pp 96-129.

Emiola, L.O. \& Delarosa, L.C. (1981). Physicochemical characteristics of yam starches. J. Food Biochem., $5: 115-130$.

Entwistle, G., Bachelor, S., Booth, E. \& Walker, K. (1998). Economics of starch production in the UK. Industrial crops and Products, 7 : 175-186.

Evans, I.D. \& Haisman, D.R. (1982). The effect of solute on the gelatinization temperature range of potato starch. Starch, $34:$ 224-231.

Evans, I.D. (1986). An investigation of starch/surfactant interaction using viscometry and differential scanning calorimetry. Starch, 38 : 227-235.

Feam, T. \& Russell, P.L. (1982). A kinetic study of bread stalling by differential scanning calorimetry. The effect of loaf volume. J. Sci. Food Agric., 33 : 537 548 .

Fleche, G. (1985). Chemical modification and degradation of starch. In Starch conversion technology. M.A. Van Beynum, \& J.A. Roels. (Eds.). Marcel Dekker Inc., New York, pp 73-99.

Flory, P.J. (1953). Pninciples of polymer Chemistry. Cornell University press, New York. 
Forrest, B. (1992). Identification and quantification of hydroxypropylation of starch by FT-IR. Starch, $44:$ 179-183.

Franco, C.M.L., Preto, S.J, Ciacco, C.F. \& Tavares, D.Q. (1988). Studies on the susceptibility of granular cassava and com starches to enzymatic attack. Part 2. Study of the granular structure of starch. Starch, $40: 29-32$.

Franco, C.M.L. \& Ciacco, C.F. (1992). Factors that affect the enzymatic degradation of natural starch granules- Effect of the size of the granules. Starch, $44: 422-426$.

Franco, C.M.L. , Ciacco, C.F. and Tavares, D.Q. (1995). Effect of heat-moisture treatment on the enzymatic susceptibility of corn starch granules. Starch, 47 : 223-228.

French, D. (1972). Fine structure of starch and its relationship to the organization of starch granules. J. Jpn. Soc. Starch Sci., $19: 8-33$.

French, A.D. \& Murphy, V.G. (1977). Computer modeling in the study of starch. Cereal Foods World, 22 : 61-70.

French, D.(1984). Organization of starch granules. In Starch. Chemistry and Technology. Vol 2. D.M. Whiltler, J.N. BeMiller \& E.F. Paschall. (Eds.). Academic Press, New York, pp 183-247.

Galliard, T. (1983). Starch-lipid complexes and other non-starch components of starch granules in cereal grains. In Recent advances in Phytochemistry. Vol 17. J.L. Peter \& F.A. Loewus. (Eds.). Plenum Press, New York, pp 111-136.

Galliard, T. \& Bowler, P. (1987). Morphology and composition of starch. In Starch : properties and potential. Vol 13. T. Galliard. (Ed.). John Wiley \& sons, New York, pp 55-78.

Germani, R., Ciacco, C.F. \& Rodriguez-Amaya, D.B. (1983). Effect of sugars, lipids and type of starch on the mode and kinetics of retrogradation of concentrated corn starch gels. Starch, 35 : 377-381.

Gernat, C., Radosta, S., Damaschun, G. \& Schierbaum, F. (1990). Supramolecular structure of legume starches revealed by $\mathrm{X}$-ray scattering. Starch, 42 : 175-187. 
Gernat, C., Radosta, S., Anger, H. \& Damaschun, G. (1993). Crystalline parts of three different conformations detected in native and enzymatically degraded starches. Starch, 45 : 309-314.

Gibinski, M., Palasinski, M. \& Tomaski, P. (1993). Physicochemical properties of defatted oat starch. Starch, $45: 354-357$.

Gidley, M.J.(1987). Factor affecting the crystalline type (A-C) of native starches and model compounds : A rationalization of observed effects in terms of polymorphic structures. Carbohydr. Res., 161: 301-304.

Gidley, M.J. \& Bociek, S.M. (1988). ${ }^{13} \mathrm{C}$ CP / MAS NMR studies of amylose inclusion complexes, cyclodextrins, and the amorphous phase of starch granules : Relationship between glycosidic linkage conformation and Solidstate ${ }^{13} \mathrm{C}$ chemical shifts. J. Am. Chem. Soc., $110: 3820-3829$.

Gidley, M.J. (1989). Molecular mechanism underlying amylose aggregation and gelatinization. Macromolecules, $22: 351-358$.

Gidley, M.J. \& Bulpin, P.V. (1989). Aggregation of amylose in aqueous systems : the effect of chain length on phase behavior and aggregation kinetics. Macromolecules, 22 : 341-346.

Gidley, M.J. (1992). Structural order in starch granules and its loss during gelatinization. In Gums and stabilizers for the food industry 6. G.O. Phillips, P.A. Williams \& D.J. Wedlock. (Eds.). IRL Press, Oxford, pp 87-92.

Goodfellow, B.J. \& Wilson, R.H. (1990). A Fourier transform IR study of the gelation of amylose and amylopectin. Blopolymers, $30: 1183-1185$.

Goshima, G., Abe, M., Sato, N., Ohashi, K. \& Tsuge, H. (1985). Amylographic reproducibility of defatted potato starch by re-introduction of lipids. Starch, 37 : $10-14$.

Gough, B.M. \& Pybus, J.N. (1971). Effect on the gelatinization temperature of wheat starch granules of prolonged treatment with water at 50C. Starch, 23 : 210-214.

Greenwood, C.T. \& Thompson, J. (1962). Physicochemical studies on starches. XXIV. Fractionation and characterization of starches of various plant origins. J. Chem. Soc., $42: 222-229$ 
Gudmundsson, M. \& Eliasson, A.C. (1989). Some physicochemical properties of oat starches extracted from varieties with different oil contents. Acta Agric. Scand. 39 : 101-112.

Gudmundsson, M. \& Eliasson, A.C. (1991). The effects of water soluble arabinoxylans on gelatinization and retrogradation of starch. Starch, $43: 5$. 10.

Hahn, D.E. \& Hood, L.F. (1980). Physical characteristics of $\alpha$-amylase hydrolysates from unmodified and chemically modified waxy maize starches. J. Food Sci., $45: 518-522$.

Hahn, D.E. \& Hood, L.F. (1987). Factors influencing corn starch lipid complexing. Cereal Chem., $64: 81-85$

Hargin, K.D. and Morrison, W.R. (1980). The distribution of acy! lipids in the germ of four wheat varieties. J. Sci. Food Agric., 31 : 877-888,

Hizukuri, S., Fujii, M. \& Nikuni, Z. (1960). The effect of inorganic ions on the crystallization of amylodextrins. Biochim. Biophys. Acta, $40: 346-348$.

Hizukuri, S. (1969). The effect of environmental temperature of plants on the physicochemical properties of their starches. J. Jpn. Soc. Starch Sci., 17 : 73-88.

Hizukuri, S., Takeda, Y., Yasuda, M. \& Suzuki, A. (1981). Multi-branched nature of amylose and the action of debranching enzymes. Carbohydr. Res., 94 : 205-213.

Hizukuri, S., Kaneko, T. \& Takeda, Y. (1983). Measurements of the chain length of amylopectin and its relevance to the origin of crystalline polymorphism. Biochim. Biophys. Acta, 760 : 188-191.

Hizukuri, S. (1986). Polymodal distribution of the chain length of amylopectin and its significance, Carbohydr. Res., $147: 342-347$.

Hizukuri, S. (1996). Starch : Analytical aspects. In Carbohydrates in Food. A.C. Eliasson. (Ed.). Marcel Dekker Inc., New York, pp 347-429.

Hofreiter, B.T. (1987). Miscellaneous modifications. In Modified starches : Properties and uses. O.B. Wurzburg. (Ed.). CRC Press Inc., Florida, pp 180196. 
Holm, J., Bjorck, I., Ostrowska, S, Eliasson, A.C., Asp, N.G., Larsson, K. \& Lundquist, I. (1983). Digestibility of amylose - lipid complexes in vitro and in vivo. Starch, $35: 294-297$.

Hood, L.F. \& Mercier, C. (1978). Molecular structure of unmodified and chemically modified manioc starches. Carbohydr. Res., 61 : 53-66.

Hood, L.F. (1982). Current concepts of starch structure. In Food Carbohydrates. D.R Lineback \& G.E. Ingett. (Eds.). AVI Publishers Company, Connecticut. pp 217-236.

Hoover, R. \& Hadziyev, D. (1981). Characterization of potato starch and its monoglyceride complexes. Starch, $33: 290-300$.

Hoover, R. \& Sosulski, F.W. (1985). Studies on the functional characteristics and digestibility of starches from Phaseolus vulgaris biotypes. Starch, 37 : 181191.

Hoover, R. \& Sosulski, F. W. (1986). Effect of cross linking on functional properties of legume starches. Starch, 38 : 149-155.

Hoover, R., Hannouz, D. \& Sosluski, F.W. (1988). Effect of hydroxypropylation on thermal properties, starch digestibility and freeze-thaw stability of field pea (Pisum sativum cv Trapper) starch. Starch, $40: 383-387$.

Hoover, R., Swamidas, G. \& Vasanthan, T. (1993). Studies on the physicochemical properties of native, defatted and heat-moisture treated pigeon pea (Cajanus cajan L) starch. Carbohydr. Res., 246 : 185-203.

Hoover, R. \& Vasanthan, T. (1994a). Effect of heat-moisture treatment on the structure and physicochemical properties of cereal, legume and tuber starches. Carbohydr. Res., 252 : 33-53.

Hoover, R. \& Vasanthan, T. (1994b). The flow properties of native, heat-moisture treated and annealed starches from wheat, oat, potato and lentil. J. Food Biochem., 18 : 67-82.

Hoover, R., Vasanthan, T., Senanayake, N.J. \& Martin, A.M. (1994). The effect of defatting and heat-moisture treatment on the retrogradation of starch geis from wheat, oat, potato and lentil. Carbohydr. Res., 261 : 13-24. 
Hoover, R. \& Manuel, H. (1996a). Effect of heat-moisture treatment on the structure and physicochemical properties of legume starches. Food Res. Int., $29: 731-750$.

Hoover, R. \& Manuel, H. (1996b). The effect of heat-moisture treatment on the structure and physicochemical properties of normal maize, waxy maize, dull waxy maize and amylomaize V starches. J. Cereal Sci., 23 : 153-162.

Horii, F., Yamamoto, H., Hiray, A. \& Kitamaru, R. (1987). Structural study of amylose polymorphs by cross polarization magic angle spinning ${ }^{13} \mathrm{C}$ NMR spectroscopy. Carbohydr. Res., $160: 29-40$.

Hoseney, R.C., Lineback, D.R. \& Seib, P.A. (1978). Role of starch in baked goods. Baker's Dig., 52 : 11-40.

Hoseney, R.C., Zeleznak, K.J. \& Yost, D.A. (1986). A note on the gelatinization of starch. Starch, $38: 407-409$.

Huang, J.J. \& White, P.J. (1993). Waxy corn starch : Monoglyceride interaction in a model system. Cereal Chem., $70: 42-47$.

I'Anson, K.J., Miles, M.J., Morris, V.J. \& Ring, S.G. (1988). A study of amylose gelation using a synchrotron X-ray source. Carbohydr. Polym., 8 : 45-53.

Imberty, A., Chanzy, H., Perez, S. Buleon, A. \& Tran, V. (1987). New three dimensional structure for A-type starch. Macromolecules, 20 : 2634-2636.

Imberty, A., Chanzy, H., Perez, S. Buleon, A. \& Tran, V. (1988). The double helical nature of the crystalline part of A-starch. J. Mol. Biol., 201 : 365-378.

Imberty, A. \& Perez, S. (1988). A revisit to the three dimensional structure of B type starch. Biopolymers, 27 : 1205-1221.

Imberty, A., Buleon, A., Tran, V. \& Perez, S. (1991). Recent advances in knowledge of starch structure. Starch, $43: 375-384$.

Imberty, A. \& Perez, S. (1996). Structural features of starch. Carbohydrates in Europe, $15: 17-21$.

Jacobson, M.R., Obanni, M. \& BeMiller, J.N. (1997). Retrogradation of starches from different botanical sources. Cereal Chem., $74: 511-518$. 
Jacobson, M.R. \& BeMiller, J.N. (1998). Method for determining the rate and extent of accelerated starch retrogradation. Cereal Chem., $75: 22-29$.

Jane, J. \& Robyt, J.F. (1984). Structure studies of amylose -V complexes and retrograded amylose by action of $\alpha$-amylases, and a new method for preparing amylodextrins. Carbohydr. Res., 132 : 105-118.

Jane, J., Craig. S.A.S., Seib, P.A. \& Hoseney, R.C. (1986). Characterization of granular cold water soluble starch. Starch, $38: 258-263$.

Jane, J., Xu, A., Radosavljevic, M. \& Seib, P.A. (1992). Location of amylose in normal starch granules. I. Susceptibility of amylose and amylopectin to cross linking reagents. Cereal Chem., $69: 405-409$.

Jane, J. (1997a). Starch functionality in food processing. In Starch : Structure and functionality. P.J. Frazier, A.M. Donald \& P. Richmond. (Eds.). The Royal Society of Chemistry, Cambridge, pp 26-35.

Jane, J. (1997b). Structural aspects of starch granule. In Starch 96 : the book, Proceedings of Symposium Starch 96. H. Van Doren \& N. Van Swaaij. (Eds.). Carbohydrate Research Foundation, Noordiwijkerhout, pp 37-46.

Jang. J.K. \& Pyun, Y.R. (1996). Effect of moisture content on melting of wheat starch. Starch, $48: 48-51$.

Jang, J.K. \& Pyun, Y.R. (1997). Effect of moisture level on the crystallinity of wheat starch aged at different temperatures. Starch, $49: 272-277$.

Jankowski, T. \& Rha, C.K. (1986). Retrogradation of starch in cooked wheat. Starch, 38 : 6-9.

Jansson, C., Puthigae, S. Sun, C. \& Grenthe, C. (1995). Genetically modified starch as an industrial raw material. J. Mol. Sci. Pure Applied Chem., A 32 : 895-898.

Jarowenko, W. (1987). Acetylated starch and miscellaneous organic esters. In Modified starches: Properties and uses, O.B. Wurzburg. (Ed.). CRC Press Inc, Florida, pp 55-77.

Jenkins, P.J., Cameron, R.E. \& Donald, A.M. (1993). A universal feature in the structure of starch granules from different botanical sources. Starch, $45: 417$ 420. 
Jenkins, P.J. \& Donald, A.M. (1995). The influence of amylose on starch granule structure. Int. Biol. Macromol., 17 : 315-321.

Johnson, D.P. (1969). Spectrophotometric determination of the hydroxypropyl groups in starch ethers. Anal. Chem., 41, 859-860.

Kainuma K. \& French, D. (1972). Naegeli amylodextrin and its relationships to starch granule structure. II. Role of water in crystallization of B-starch. Biopolymers, 11: 2241-2250.

Kalichevsky, M.T., Orford, P.D. \& Ring, S.G. (1990). The retrogradation and gelation of amylopectin from various botanical sources. Carbohydr. Res., 198 :49-55.

Kasemsuwan, T. \& Jane, J. (1994). Location of amylose in normal starch granules. II. Location of phosphodiester cross linking revealed by phosphorous 31 Nuclear Magnetic Resonance. Cereal Chem., 71 : 282-287.

Kassenbeck, P. (1978). Contribution to the knowledge on distribution of amylose and amylopectin in starch granules. Starch, 30:40-46.

Katsuta, K. Nishimura, A. \& Miura, M. (1992a). Effect of saccharides on stabilities of rice starch gels. I. Mono- and disaccharides. Food Hydrocolloids, 6 : 387-398.

Katsuta, K. Nishimura, A. \& Miura, M. (1992b). Effect of saccharides on stabilities of rice starch gels. 2. Oligosaccharides. Food Hydrocolloids, 6 : 399-408.

Keetels, C.J.A.M., Oostergetel, G.T. \& van Viet, T. (1996). Recrystallization of amylopectin in concentrated starch gels. Carbohydr. Polym., 30 : 61-64.

Kesler, C.C \& Hjermstad, E.T. (1950), Preparation of starch ethers in original granule form, US Patent , 2, 516,633.

Kim, C.S. \& Walker, C.E. (1992). Effects of sugars and emulsifiers on starch gelatinization evaluated by differential scanning calorimetry. Cereal Chem., $69: 212-217$.

Kim, H.R., Hermansson, A.M. \& Eriksson, C.E. (1992). Structural characteristics of hydroxypropyl potato starch granules depending on their molar substitution. Starch, 44 : 111-116. 
Kim, H.R. \& Eliasson, A.C. (1993). Changes in theological properties of hydroxypropyl potato starch pastes during freeze thaw treatments. Il. Effect of molar substitution and cross linking. J. Text. Stud., 24 : 199-213.

Kim, H.R., Muhrbeck, P. \& Eliasson, A.C. (1993). Changes in theological properties of hydroxypropyl potato starch pastes during freeze thaw treatment. III. Effect of cooking conditions and concentration of the starch pastes. J. Sci. Food Agric., 61 : 109-116.

Kim, J., Kim, W. \& Shin, M. (1997). A comparative study on retrogradation of rice starch gels by DSC, $X$-ray and $\alpha$-amylase. Starch, 49 : 71-75.

Knutson, C.A., Khoo, U., Cluskey, J.E. \& Inglett, G.E. (1982). Variation in enzyme digestibility and gelatinization behavior of corn starch granule fraction. Cereal Chem., 59 : 512-515.

Kohyama, K. \& Nishinari, K. (1991). Effect of soluble sugars on gelatinization and retrogradation of sweet potato starch. J. Agric. Food Chem., $39: 1406-$ 1410.

Kowblansky, M. (1985). Calorimetric investigation of inclusion complexes of amylose with long chain aliphatic compounds containing different functional groups. Macromolecules, 18 : 1776-1779.

Krog, N. \& Jensen, N.B. (1970). Interaction of monoglycerides in different physical states with amylose and their anti-firming effects in bread. J. Food Technol., $5: 77-87$.

Krog, K. (1971), Amylose complexing effect of food grade emulsifiers. Starch, 23 :206-210.

Krusi, H. von \& Neukom, H. (1984). Untersuchunger ttber die retrogradation der stärke in konzentrierten weizestarkegelen. Teil I. Herstellung konzentrierter gele, einflub der starkekonzentration and herstellungsbedingungen auf die retrogradation der starke. Starch, $36: 40-45$.

Kulp, K \& Lorenz, K. (1981) Heat-moisture treatment of starches. I Physicochemical properties. Cereal Chem., 58 : 46-48.

Lagendiijk, J. \& Pennings, H.J. (1970). Relation between complex formation of starch with monoglycerides and the firmness of bread. Cereal Sci. Today, 15 : 354-365. 
Larsson, K. (1983). Physical state of lipids and their technical effects in baking. In Lipids in Cereal Technology. P.J. Barnes. (Ed.). Academic Press, London. pp 237-251.

Larsson, K. \& Dejmek, P. (1990). Crystal and liquid crystal structures of lipids. In Food Emulsions. K. Larsson \& E. Friberg. (Eds.). Marcel Dekker Inc., New York, pp 97-125.

Laszlò, E., Hollo, J., Hoschke, A. \& Sarosi, G. (1978). A study by means of lactone inhibition of the role of a 'half chair' glycosyl conformation at the active center of amylolytic enzymes. Carbohydr. Res., 61 : 387-394.

Le Bail, P., Bizot, H. \& Buleon, A. (1993). 'B' to 'A' type phase transition in short amylose chains. Carbohydr. Polym., 21 ; 99-104.

Leech, H.W., McCowen, L.D. \& Schoch, T.J. (1959). Structure of the starch granule. I. Swelling and solubility patterns of various starches. Cereal Chem., 36 : $534-544$.

Leegwater, D.C. \& Luten, J.B. (1971). A study of the in vitro digestibility of hydroxypropyl starches by pancreatin. Starch, $23: 430-432$.

Lelievre, J. (1976). Theory of gelatinization in a starch-water-solute system. Polymer, 17 : 854-858.

Lelievre, J. (1992). Thermal analysis of carbohydrates as illustrated by aqueous starch systems. In Developments in Carbohydrate Chemistry. R.J. Alexander \& H.F. Zobel. (Eds.). American Association of Cereal Chemists, Minnesota, pp 137-161.

Leloup, V.M., Colonna, P. \& Buleon, A. (1991). Influence of amyloseamylopectin ratio on gel properties. J. Cereal Sci., 13 : 1-13.

Leloup, V.M., Colonna, P., Ring, S.G., Robert, K. \& Wells, B. (1992). Microstructure of amylose gels. Carbohydr. Polym., 18 : 189-197.

Lilford, P.J. \& Morrison, A. (1997). Structure / function relationship of starches in Food. In Starch : Structure and functionality. P.J. Frazier, P. Richmond \& A.M. Donald. (Eds.). The Royal Society of Chemistry, Cambridge, pp 1-8.

Lineback, D.R. (1984). The starch granule : Organization and properties. Baker's Dig., $58: 16-21$. 
Lineback, D.R. \& Rasper, V.F. (1988). Wheat carbohydrates. In Wheat : Chemistry and Technology. Y. Pomeranz. (Ed.). American Association of Cereal Chemists, Minnesota, pp 277-372.

Liu, H., Lelievre, J. \& Ayoung-Chee, W. (1991). A study of starch gelatinization using differential scanning calorimetry, $X$-ray and birefringence measurements. Carbohydr. Res., $210: 79-87$.

Longton, J. \& LeGrys, G.A. (1981). Differential scanning calorimetry studies on the gelatinization of aging gels. Starch, $33: 410-414$.

Lonkhuysen, V. \& Blaknestijn, J. (1976). Interaction of monoglycerides with Starches. Starch, $26: 337-342$.

Lorenz, K. \& Kulp. K. (1981). Heat-moisture treatment of starches. II. Functional properties and baking potential. Cereal Chem., $58: 49-52$.

Lorenz, K. \& Kulp, K. (1982). Cereal and root starch modification by heatmoisture treatment. I. Physicochemical properties. Starch, $34: 50-54$.

Lorenz, K. \& Kulp, K. (1983). Physico-chemical properties of defatted heatmoisture treated starches. Starch, 35 : 123-129.

Lowry, G.D.A., Sargeant, J.G. \& Schofield, J. D. (1981).Wheat starch granule protein : The isolation and characterization of a salt-extractable protein from starch granules. J. Sci. Food Agric., 32 : 371-377.

Lu, S., Chen, L. \& Lii, C. (1997). Correlation between the fine structure, physicochemical properties and retrogradation of amylopectin from Taiwan rice varieties. Cereal Chem., $74: 34-39$.

Lund, D. (1984). Influence of time, temperature, moisture, ingredients and processing condition on starch gelatinization. CRC Crit. Rev. Food Sci. Nutr. $20: 249-272$.

Manners, D.J. \& Matheson, N.K. (1981). The fine structure of amylopectin. Carbohydr. Res., 90 : 99-110.

Manners, D.J. (1985). Structural analysis of starch components by debranching enzymes. In New approaches to research on cereal carbohydrates. R.D. Hill \& L. Munck. (Eds.). Elsevier Science Publishers, Amsterdam, pp 45-60. 
Marsden, W.L. \& Gray, P.P. (1986). Enzymatic hydrolysis of cellulose in ligno cellulosic materials. CRC Crit. Rev. Biotechnol., 3 : 235-276.

Marshall, J.J. \& Whelan, W.J. (1974). Multiple branching in glycogen and amylopectin. Arch. Biochem. Biophys., $161: 234-237$.

Mclver, R.G., Axford, D.W.E., Colwell, K.H. \& Elton, G.A.H. (1968). Kinetic study of the retrogradation of gelatinized starch. J. Sci. Food Agric., $19: 560-563$.

Melvin, M.A. (1979). The effect of extractable lipid on the viscosity characteristics of corn and wheat starches. J. Sci. Food Agric., 30 : 731-738.

Mercier, C., Charbonnier, R. Grebaut, J. \& De La Gueriviere, J.F. (1980). Formation of amylose lipid complexes by twin-screw extrusion cooking of manioc starch. Cereal Chem., 57, 4-9.

Miles, M.J., Morris, V.J., \& Ring, S.J. (1985a). Gelation of amylose. Carbohydr. Res., $135: 257-269$.

Miles, M.J., Morris, V.J., Oxford, P.D. \& Ring, S.G. (1985b). The role of amylose and amylopectin in the gelation and retrogradation of starch. Carbohydr. Res., 135: 271-281.

Minitab, Inc. (1991). Minitab computer supplement : Statistic for business and economics. $5^{\text {th }}$ ed. Minitab Inc., State College, PA, pp 299-319.

Miura, M., Nishimura, A. \& Katsuta, K. (1992). Influence of addition of polyols and food emulsifiers on the retrogradation rate of starch. Food Structure, 11 : 225-236.

Mohd Azemi, B.M.N. \& Wootton, M. (1984). In vitro digestibility of hydroxypropyl maize, waxy maize, and high amylose maize starches. Starch, $36: 273-275$.

Mohd Azemi, B.M.N. \& Wootton, M. (1985). Action pattem of porcine pancreatic alpha amylase on hydroxypropyl derivatives of maize, waxy maize and high amylose maize starch. Starch, 37 : 50-52.

Mohd Azemi, B.M.N. \& Wootton, M. (1995). Distribution of partial digestion products of hydroxypropyl derivatives of maize (NM), waxy maize (WM) and high amylose maize (HA) starches. Starch, $47: 465-469$.

Morgan, K.R., Furneaux, R.H. \& Larsen, N.G. (1995). Solid-state NMR studies on the structure of starch granules. Carbohydr.Res., 276 : 387-399. 
Morris, V.J. (1990). Starch gelation and retrogradation. Trends in Food Sci. \& Technol., $1: 2-6$.

Morrison, W.R. (1981). Starch lipids - A reappraisal. Starch, $33: 408-410$.

Morrison, W.R. \& Milligan, T.P. (1982). Lipids in maize starch. In Maize : Recent progress in chemistry and technology, G. Inglett. (Ed.). Academic Press, New York, pp 1-18.

Morrison, W.R. \& Laignelet, B. (1983). An improved method for determining apparent and total amylose in cereal and other starches. J. Cereal Sci., $1: 9$ 20.

Morrison, W.R., Law, R.V. \& Snape, C.E. (1993a). Evidence for inclusion complexes of lipid with $\mathrm{V}$-amylose in maize, rice and oat starches. J. Cereal Sci., 18 : 107-111.

Morrison, W.R., Tester, R.F., Snape, C.E., Law, R.V. \& Gidley, M.J. (1993b). Swelling and gelatinization of cereal starches. N Some effects of lipidcomplexed amylose and free amylose in waxy and nomal barley starches. Cereal Chem., 70 : 385-391.

Muller, J.J., Gernat, C., Schultz, W., Muller, E.C., Vorwerg, W. \& Damaschun, G. (1995). Computer simulations of X-ray scattering curves : Gelation and crystallization process in amylose solutions. Biopolymers, $35: 271-288$.

Nakazawa, F., Noguchi, S., Takahashi, J. \& Takada, M. (1985). Retrogradation of gelatinized potato starch studied by differential scanning calorimetry. Agric. Bial. Chem., 49 : 953-957.

Nikuni, Z. (1969). Starch and cookery. J. Sci. Cookery, 2 : 6-10.

Nikuni, Z. (1978). Studies on starch granule. Starch, 30 : 105-111.

Oostergetel, G.T. \& Van Bruggen, E.F.J. (1989). On the origin of a low angle spacing in starch. Starch, $41: 331-335$.

Oostergetel, G.T. \& Van Bruggen, E.F.J. (1993). The crystalline domains in potato starch granules are arranged in a helical fashion. Carbohydr. Polym., $21: 7-12$. 
Orford, P.D., Ring, S.G., Carrol, V., Miles, M.J. \& Morris, V.J. (1987). The effect of concentration and botanical source on the gelation and retrogradation of starch. J. Sci. Food Agric., 39 : 169-177.

Orford, P.D., Parker, R. \& Ring, S.G. (1993). The functional properties of extrusion cooked waxy maize starch. J. Cereal Sci., 18 : 277-286.

Osman-Ismail, F. (1972). The formation of inclusion compounds of starches and starch fractions. Diss. No 4829, Juris Druck, Zurich.

Ostergard, K., Bjorck, I. \& Gunnarsson, A. (1988). A study of native and chemically modified potato starches. Part 1. Analysis and enzymatic availability in vitro. Starch, $40: 58-66$.

Paschall, E.F. (1967). Production and uses of cationic starches. In Starch : Chemistry and Technology. Vol 2 . R.L. Whistler \& E.F. Paschall. (Eds.). Academic Press, New York, pp 403-422.

Perez, S., Imberty, A. \& Scaringe, R.P. (1990). Computer modeling of carbohydrate molecules. In ACS Symposium series 430. A.D. French \& J.W. Brady. (Eds.). American Chemical Society, Washington D.C., pp 281-299

Pfannemuller, B. (1987). Influence of chain length of short monodisperse amylose on the formation of A- and B-type X-ray diffraction patterns. Int. $\mathrm{J}$. Biol. Macromol., 9 : 105-108.

Preiss, J. \& Lori, C. (1980). Starch biosynthesis and degradation. In The Biochemistry of plants : a comprehensive treatise. Vol 3. J. Preiss. (Ed.). Academic Press, New York, pp 371-423.

Prokopowich, D.J. \& Biliaderis, C.G. (1995). A comparative study of the effect of sugars on the thermal and mechanical properties of concentrated waxy maize, wheat, potato and pea starch gels. Food Chem., $52: 255-262$.

Rege, A. \& Pai, J.S. (1996). Thermal and freeze that properties of starch of chickpea (Cicer arietinum). Food Chem., 57 : 545-547.

Riisom, T., Krog, K. \& Eriksen, J. (1984). Amylose complexing capacities of cisand trans- unsaturated monoglycerides in relation to their functionality in bread. J. Cereal Sci., 2 : 105-118.

Ring, S.G. (1985). Some studies on starch gelation. Starch, $37: 80-83$. 
Ring. S.G. (1987). Molecular interactions in aqueous solution of the starch polysaccharides. A review. Food Hydrocolloids, 1 : 449-454.

Ring, S.G., Colonna, P., I'Anson, K.J., Kalichevsky, M.T., Miles, M.J., Morris, V.J. \& Oxford, P.D. (1987). The gelation and crystallization of amylopectin. Carbohydr. Res., $163: 277-293$.

Ring. S.G., Gee, J.M., Whittam, M., Orford, P.D. \& Johnson, I.T. (1988). Resistant starch : its chemical form in foodstuffs and effects on digestibility in vitro. Food Chem., 28 : 97-109.

Robin, J.P., Mercier, C., Charbonniere, R. \& Guilbot, A. (1974). Lintnerized starches. Gel filtration and enzymatic studies of insoluble residues from prolonged acid treatment of potato starch. Cereal Chem., $51: 389-406$.

Robyt, J.F. \& French, D. (1970). The action pattern of porcine pancreatic $\alpha$ amylase in relationship to the substrate binding site of the enzyme. J. Biol. Chem., 245 : 3917-3927.

Robyt, J.F. (1984). Enzymes in the hydrolysis and synthesis of starch. In Starch : Chemistry and Technology. R.L. Whistler, J.N. BeMiller \& E.F. Paschall. (Eds.). Academic Press, New York, pp 87-123.

Roulet, P.H., Mac Innrs, W.M., Wursch, P., Sanchez, R.M. \& Raemy, A. (1988). A comparative study of the retrogradation kinetics of gelatinized wheat starch in gel and powder form using X-ray, differential scanning calorimetry and dynamic mechanical analysis. Food Hydrocolloids, 2 : 381-396.

Russell, P.L. (1987). Aging of gels from starches of different amylose I amylopectin content studied by differential scanning calorimetry. J. Cereal Sci., $6: 147-158$.

Russell, P.L. \& Oliver, G. (1989). The effect of $\mathrm{pH}$ and $\mathrm{NaCl}$ content on starch gel aging. A study by Differential scanning calorimetry and rheology. J. Cereal Sci., $10: 123-138$.

Sair, L. (1964). Heat-moisture treatment of starches. In Methods in Carbohydrate Chemistry. Vol 4. R.L. Whistler. (Ed.). Academic Press, New York, p 283.

Sair, L. (1967). Heat-moisture treatment of starch. Cereal Chem., $44: 8-26$.

Sajjan, S.U. \& Rao, M.M.R. (1987). Effect of hydrocolloids on the theological properties of wheat starch. Carbohydr. Polym., $7: 395-402$. 
Schoch, T.J. (1964). lodometric determination of amylose. In Methods in Carbohydrate chemistry. Vol 4. R.L. Whistler. (Ed.). Academic Press, New York, pp 157-160.

Seneviratne, H.D. \& Biliaderis, C.G. (1991). Action of $\alpha$-amylase on amyloselipid complex superstructure. J. Cereal Sci., $13: 129-143$.

Senior, M.B. \& Hamori, E. (1973). Investigation of the effect of amylose / / 2 complexation on the conformation of amylose in aqueous solution. Biopolymers, 12 : 65-67.

Seow, C.C. \& Thevamalar, K. (1993). Internal plasticization of granular rice starch by hydroxypropylation : Effect on phase transitions associated with gelatinization. Starch, $45: 85-88$.

Seow, C.C., Teo, C.H. \& Nair, K.V. (1996). A differential scanning calorimetry study of the effects of sugars on thermal properties of rice starch gels before and after aging. $J$. Thermal Anal., 47 : 1201-1212.

Shiotsubo, T. \& Takahashi, K. (1984). Differential thermal analysis of potato starch gelatinization. Agric. Biol. Chem., 48 : 9-17.

Silverio, J., Svensson, E., Eliasson, A.C. \& Olofsson, G. (1996). Isothermal microcalorimetric studies on starch retrogradation. J. Thermal Anal., 47, 1179-1200.

Slade, L \& Levine, H. (1987). Recent advances in starch retrogradation. In Recent Developments in Industrial Polysaccharides. S.S. Stivala, V. Cresenzi \& I.C.M. Dea. (Eds.). Gordon \& Breach, New York, pp 387-430.

Slade, L. \& Levin, H. (1988). Non-equilibrium melting of native granular starch. Part 1. Temperature location of the glass transition associated with gelatinization of A-type cereal starch. Carbohydr. Polym., 8 : 183-208.

Solarek, D.B. (1987). Cationic starches. In Modified starches: Properties and uses. O.B. Wurzburg. (Ed.). CRC Press Inc., Florida, pp 113-129.

Staudinger, H. \& Husemann, E. (1937). Highly polymerized compounds. The constitution of starch. Anal Chem., 527 : 195-197.

Stute, R. \& Konieczny-Janda (1983). DSC investigations of starches. Starch, 35 : 340-347. 
Stute, R. (1992). Hydrothermal modification of starches: The difference between annealing and heat moisture treatment. Starch, $44: 205-214$.

Svensson, E. \& Eliasson, A.C. (1995). Crystalline changes in native wheat and potato starches at intermediate water levels during gelatinization. Carbohydr. Polym., 26 : 171-176.

Swinkells, J.J. (1985). Composition and properties of commercial native starches. Starch, 37 : 1-5.

Takahashi, S. \& Seib, P.A. (1988). Paste and gel properties of prime corn and wheat starches with and without native lipids. Cereal Chem., 65 : 474-483.

Takahashi, S., Maningat, C.C. \& Seib, P.A. (1989). Acetylated and hydroxypropylated wheat starch : Paste and gel properties compared with modified maize and tapioca starches. Cereal Chem., 66 : 499-506.

Takeda, Y., Tokunaga, N., Takeda, C. \& Hizukuri, S. (1986). Physicochemical properties of sweet potato starches. Starch, $38: 345-350$.

Teitelbaum, R.C., Ruby, S.L. \& Marks, T.J. (1978). Amylose -iodine complex formation. J. Am. Chem. Soc., $100: 3215-3218$.

Tester, R.F. \& Morrison, W.R. (1990a). Swelling and gelatinization of cereal starches. I. Effect of amylopectin, amylose and lipids. Cereal Chem., 67 : 551-557.

Tester, R.F. \& Morrison, W.R. (1990b). Swelling and gelatinization of cereal starches. II. Waxy rice starch. Cereal Chem., $67 ; 558-563$.

Thoma, J.A. (1968). A possible mechanism for amylase catalysis. J. Theoret. Biol., 19 : 297-310.

Tuschhoff, J.V. (1986), Hydroxypropylated starches. In Modified starches: Properties and uses. O.B. Wurzburg. (Ed.). CRC Press Inc., Florida, pp 8996.

Van Lonkhuysen, H. \& Blankestijn, J. (1974). Interaction of monoglycerides with starches. Starch, $26: 337-342$.

Van Soest, J.J.G., de Wit, D., Toumois, H. \& Vliegenthart, J.F.G. (1994). Retrogradation of potato starch as studied by Fourier Transform Infrared spectroscopy. Starch, $46:$ 453-457. 
Van Soest, J.J.G., Toumois, H., de Wit, D. \& Vliegenthart, J.F.G. (1995). Shortrange structure in (partially) crystalline potato starch determined with attenuated total reflectance Fourier-transform IR spectroscopy. Carbohydr. Res., 279 : 201-214.

Vasanthan, T. \& Hoover, R. (1992a). Effect of defatting on starch structure and physicochemical properties. Food Chem., 45 : 337-347.

Vasanthan, T. \& Hoover, R. (1992b). A comparative study of the composition of lipids associated with starch granules from various botanical sources. Food Chem., 43 : 19-29.

Wang, W.J. \& Jane, J. (1994). Correlation between glass transition temperature and starch retrogradation in the presence of sugars and maltodextrins. Cereal Chem., 71 : 527-531.

Wang, W.J., Powell, A.D. \& Oates, C.G. (1995). Patterns of hydrolysis in raw sago starch : effects on processing history. Carbohydr. Polym., 26 : 91-97.

Ward, K.E.J., Hoseney, R.C. \& Seib, P.A. (1994). Retrogradation of amylopectin from maize and wheat starches. Cereal Chem., 71 : 150-155.

Whittam, M.A., Noel, T.R. \& Ring, S.G. (1990). Melting behavior of A - type crystalline starch. Int. J. Biol. Macromol., $12: 359-362$.

Whittam, M.A., Noel, T.R. \& Ring. S.G. (1991). Melting and glass transition of starch polysaccharides, In Food polymers, gels and colloids. E. Dickinson. (Ed.). Royal Society of Chemistry, Cambridge, pp 277-288.

Wilson, R.H., Goodfellow, B.J. \& Belton, P.S. (1991). Comparison of Fourier transform mid infrared spectroscopy and near infrared reflectance spectroscopy with differential scanning calorimetry for the study of the stalling of bread. J. Sci. Food Agric., $54: 471-483$.

Wootton, M. \& Bamunuarachchi, A. (1979). Application of differential scanning calorimetry to starch gelatinization. I. Commercial native and modified starches. Starch, $31: 201-204$.

Wootton, M. \& Chaudhry, M.A. (1981). In vitro digestion of hydroxypropyl derivatives of wheat starch. Starch, 33 : 135-137.

Wootton, M. \& Manatsathit, A. (1983). The influence of molar substitution on the water binding capacity of hydroxypropyl maize starches. Starch, $35: 92-94$. 
Wootton, M. \& Manatsathit, A. (1984). The influence of molar substitution on the gelatinization of hydroxypropyl maize starches. Starch, $36: 207-208$.

Wootton, M. \& Haryadi, (1992). Effects of starch type and preparation conditions on substituent distribution in hydroxypropyl starches. J. Cereal Sci., $15: 181$ 184.

Wu, H.C.H. and Sarko, A. (1978a). The double-helical molecular structure of crystalline B- amylose. Carbohydr. Res., 61: 7-26.

Wu, H.C.H. \& Sarko, A. (1978b). The double helical molecular structure of Aamylose. Carbohydr. Res., $61: 27-40$.

Wu, J.Y. \& Eads, T.M. (1993). Evolution of polymer mobility during aging of gelatinized waxy maize starch : a magnetization transfer ' $\mathrm{H}$ NMR study. Carbohydr. Polym., $20: 51-60$.

Wunderlich, B. (1976). Macromolecular Physics : Crystal nucleation, growth, annealing, Vol 2. Academic Press, New York.

Wursch, P., Del Vedovo. S.D. \& Koellreutter, B. (1986). Cell structure and starch nature as key determinants of the digestion rate of starch in legumes. Am. J. Clin. Nutr., $43: 25-29$.

Wursh, P. \& Gumy, D. (1994). Inhibition of amylopectin retrogradation by partial beta-amylosis. Carbohydr. Res., 256 : 129-137.

Wurzburg, O.B. (1987), Introduction. In Modified starches : Properties and uses. O.B. Wurzburg. (Ed.). CRC Press Inc., Florida, pp 3-16.

Yamaguchi, M., Kainuma, K. \& French, D.J. (1979). Electron microscopy observations of waxy maize starch. Ultrastr. Res., $69: 249-261$.

Yeh, A. \& Yeh, S. (1993). Some characteristics of hydroxypropylated and cross linked rice starch. Cereal Chem., $70: 596-601$.

Yeh, A. \& Jeng-Yune, L. (1996). Kinetics of phase transitions of native, cross linked and hydroxypropylated rice starches. Starch, 48 : 17-21.

Yook, C., Pek, U. \& Park, K. (1993). Gelatinization and retrogradation characteristics of hydroxypropylated and cross linked rices. J. Food Sci., 58 : 405-407. 
Yost, D.A. \& Hoseney, R.C. (1986). Annealing and glass transition of starch. Starch, 38 : 289-292.

Yuan, R.C. Thompson, D.B. \& Boyer, C.D. (1993). Fine structure of amylopectin in relation to gelatinization and retrogradation behavior of maize starches from 3 wax- containing genotypes in 2 inbred lines. Cereal Chem., $70: 81$ 89.

Yuan, R.C. \& Thompson, D.B. (1998). Rheological and thermal properties of aged starch pastes from three waxy maize genotypes. Cereal Chem., 75 : 117-123.

Xu, A. \& Seib, P.A. (1997). Determination of the level and the position of substitution in hydroxypropylated starch by high resolution ${ }^{1} \mathrm{H}$ NMR spectroscopy of alpha-limit dextrins. J. Cereal Sci., 25 : 17-26.

Zeleznak, K.J. \& Hoseney, R.C. (1986). The role of water in retrogradation of wheat starch gels and bread crumb. Cereal Chem., 63 : 407-411.

Zeleznak, K.J. \& Hoseney, R.C. (1987). The glass transition in starch. Cereal Chem., 64 : $121-124$.

Zobel, H.F. (1988a). Starch crystal transformations and their industrial importance. Starch, $40: 1-7$.

Zobel, H.F. (1988b). Molecules to granules : A comprehensive starch review. Starch, $40: 44-50$.

Zobel, H.F., Young, S.N. \& Rocca, L.A. (1988). Starch gelatinization : an X-ray diffraction study. Cereal Chem., 65 : 443-446. 


\section{Publications in Refereed Journals}

1. Perera, C.. Hoover, R. \& Martin, A.M. (1997). The effect of hydroxypropylation on the structure and physicochemical properties of native, defatted and heat-moisture treated potato starches. Food Res. Int., $30: 235-$ 247.

2. Perera, C. \& Hoover, R. (1998). The reactivity of porcine pancreatic $\alpha$ amylase towards native defatted and heat-moisture treated potato starches before and after hydroxypropylation. Starch, (in press).

3. Perera, C. \& Hoover, R. (1998). Influence of hydroxypropylation on retrogradation properties of native, defatted and heat-moisture treated potato starches. Food Chem., (in press).

4. Chavan, U., Shahidi, F., Hoover, R. \& Perera, C. (1998). Isolation and physicochemical properties of beach pea starch. Food Chem., (in press).

5. Hoover, R., Sinnott, A.W. \& Perera, C. (1998). Physicochemical characterization of starches from amaranthus cruentus grains. J. Food Biochem., (submitted for publication).

\section{Papers in preparation}

1. Perera, C. \& Hoover, R. (1998). Influence of defatting, heat-moisture treatment and hydroxypropylaiton on the susceptibility of retrograded potato starch gels towards $\alpha$-amylase. (In preparation).

\section{Presentations}

1. Perera, C., Hoover, R. \& Martin, A.M. (1996). The influence of defatting and heat moisture treatment on the reactivity of potato starch towards hydroxypropylation. $79^{\text {th }}$ Annual conference of Canadian Society for Chemistry. June 23-26. Newfoundland, Canada.

2. Perera, C., Hoover, R. \& Martin, A.M. (1996). Effect of hydroxypropylation on the thermal characteristics of native, defatted and heat-moisture treated potato starches. $39^{\text {th }}$ Annual conference of Canadian Institute of Food Science and Technology. August 17-22. Guelph, Canada. 
3. Perera, C. \& Hoover, R. (1997). Structural and physicochemical characteristics of native, defatted and heat-moisture treated potato starch granules before and after hydroxypropylation. Annual Meeting of Institute of food technologists. June 14-18, Orlando, USA.

4. Perera, C., Hoover, R. (1997). The reactivity of alpha-amylase towards native, defatted and heat-moisture treated hydroxypropyl potato starches. Annual conference of Canadian Institute of Food Science and Technology. September 20-24. Montreal, Canada.

5. Hoover, R. \& Perera, C. (1998). Retrogradation studies on physically and chemically modified potato starches. Production and uses of starches. Association of Applied Biologists, April 6-8. Edinburgh, UK.

6. Perera, C. \& Hoover, R. (1998). Role of starch modification on retrogradation of potato starch gels. CIC-APIC Atlantic Student Chemistry Conference. May 13-15, Newfoundland, Canada.

7. Perera, C. \& Hoover, R. (1998). A comparative study of the rate and the extent of retrogradation of native, defatted and heat-moisture treated potato starches before and after hydroxypropylation. Annual Meeting of Institute of food technologists. June 20-24, Atlanta, USA.

\section{Scholarships and Awards}

1984-1988 Scholarship for Undergraduate studies, Sri Lanka

1991-1993 Scholarship for Postgraduate studies, UK

1995 -1998 Graduate Fellowship, Dept. Biochemistry, Memorial University of Newfoundland

1997 Graduate student paper award (Carbohydrate division), Annual Meeting of Institute of Food Technologists, Orlando, USA.

1998 Fellow of the Graduate School, Memorial University of Newfoundland

1998-1999 Postdoctoral Fellowship, lowa State University, USA 





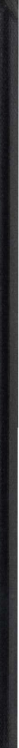

